\title{
The nuclear envelope proteins Kugelkern and Narf and their putative role in aging
}

\author{
Dissertation \\ for the award of the degree \\ "Doctor rerum naturalium (Dr.rer.nat.)” \\ in the GGNB program "Genes and Development" \\ Division of Mathematics and Natural Sciences \\ at the Georg-August-University Göttingen \\ submitted by \\ Maria Kriebel \\ from Aschersleben, Germany
}

Göttingen, January 2018

revised version October 2018 


\section{MEMBERS OF THE THESIS COMMITTEE}

\section{Prof. Jörg Großhans (Supervisor, reviewer)}

Department of Developmental Biochemistry, University of Göttingen

\section{Prof. Reinhard Schuh (Reviewer)}

Department of Molecular Developmental Biology, Max Planck Institute for Biophysical Chemistry, Göttingen

\section{Prof. Harald Hermann-Lerdon}

Department of Molecular Genetics, German Cancer Research Center, Heidelberg

\section{MEMBERS OF THE EXAMINATION BOARD}

\section{Prof. Gregor Bucher}

Department of Evolutionary Developmental Genetics, University of Göttingen

\section{Prof. Bernd Wollnik}

Department of Human Genetics, University of Göttingen

\section{PD Dr. Halyna Shcherbata}

Department of Gene expression and signaling, Max Planck Institute for Biophysical Chemistry, Göttingen

Date of the oral examination: 13.03 .2018 


\section{AFFIDAVIT}

I hereby declare that I wrote this thesis "The nuclear envelope proteins Kugelkern and Narf and their putative role in aging "on my own with no other sources and aids than indicated.

Maria Kriebel

Göttingen, 31.01.2018 


\section{TABLE OF CONTENT}

Acknowledgments .......................................................................................................................- 1 -

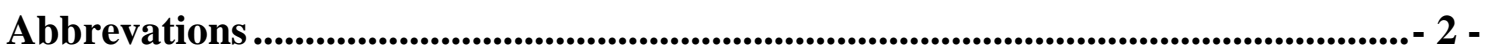

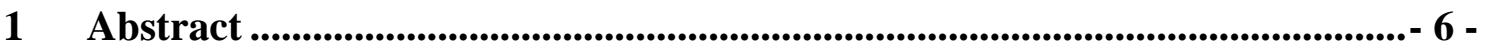

2 Introduction ..................................................................................................... 7 -

$2.1 \quad$ Nuclear lamins................................................................................... 7 -

2.2 Lamin A mutations and Huntchinson-Gilford progeria syndrome................. 9 -

2.3 Drosophila as a model to study aging .......................................................- 10 -

$2.4 \quad$ Narf - a new progeria gene? ..................................................................- 11 -

2.5 Narf like genes have a common ancestor and play a role in

Fe-S protein biogenesis ............................................................................. 12 -

2.6 Role of Narf-like genes in sensing oxygen.................................................- 13 -

$2.7 \quad$ Role of Narf-like genes in aging................................................................- 14 -

3 Aim of the study ...................................................................................................... 15 -

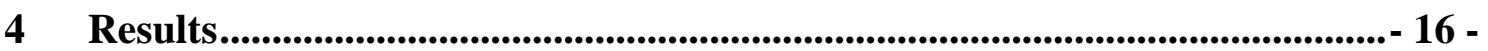

4.1 Identification of novel Kuk interaction partners..........................................- 16 -

4.1.1 Characterization of $\Delta 15$ a kuk deficient fly line...................................- 16 -

4.1.2 Nuclear elongation is missing in $\Delta 15$ embryos ....................................- 17 -

4.1.3 Gene expression is altered in kuk-depleted embryos............................- 19 -

4.1.4 Survival rate is affected in kuk-depleted animals ................................- 20 -

4.2 Identification of biochemical interaction partners of Kuk............................. 22 -

4.2.1 GFP-Kuk transgenes did not rescue the kuk loss of function phenotype- 22 -

4.2.2 Proteolytic cleavage of GFP-Kuk......................................................- 23 -

4.2.3 Nuclei solubilization promoted further degradation..............................- 26 -

4.2.4 Biochemical properties of GFP-Kuk (C567S) .....................................- 28 -

4.3 Identification of genetic interaction partners of Kugelkern.........................- 30 -

4.3.1 Morphological defects upon Kugelkern overexpression were primary independent of apoptosis.................................................- 31 -

4.3.2 Genetic interaction partners of Kuk remain undiscovered ...................- 35 -

4.4 The role of Narf in nuclear morphogenesis and aging ................................. 37 -

4.4.1 Generation of a Narf specific antibody..............................................- 37 -

4.4.2 Narf localized to the nuclear envelope ……………………………......- 39 -

4.4.3 Narf has a function during Drosophila development ............................- 41 - 
4.4.4 The lethal allele NC 38 probably resulted in nonsense-mediated decay- 43 -

4.4.5 Genomic transgenes mimicing the human patient situation................ 44 -

4.4.6 Increase of genomic copies of Narf might positively affect viability - 46 -

4.4.7 Ubiquitous overexpression of Narf had beneficial effects on lifespan- 49 -

4.4.8 Using the CRISPR approach for generating a Narf null allele........... 50 -

4.4.9 The GeneSwitch Gal4 system was used to muscle specifically express GFP in a dose dependent manner .......................................- 52 -

4.4.10 The GeneSwitch system was used to muscle specifically overexpress Narf................................................................................ 55 -

4.4.11 Overexpression of Narf increased nuclear size in muscle cells.......... 56 -

4.4.12 Muscle specific overexpression of Narf affected negative geotaxis behavior ............................................................... 58 -

4.4.13 Muscle specific overexpression of Narf affected Drosophila lifespan- 60 -

4.4.14 Knock-down of Narf did not significantly increase nuclear size in muscle cells $-63-$

4.4.15 Muscle specific knock-down of Narf during adulthood affected lifespan............................................................................ 65 -

4.4.16 Narf is essential during adult stage .................................................. 67 -

4.4.17 Gene expression is changed in Narf overexpressing or down regulating situations................................................................. 69 -

4.4.18 Klp68D is a Narf interaction partner ................................................ 71 -

4.5 Is Narf involved in aging in C. elegans? ................................................. 72 -

4.5.1 Oxygen sensitivity of $0 x y-4$ mutants ................................................ 72 -

4.5.2 oxy-4 mutants showed a developmental delay under normal conditions- 73 -

4.5.3 Unchanged muscle structure in oxy-4 mutants ................................. 74 -

4.5.4 Nuclei morphology and Ce-lamin farnesylation were not affected

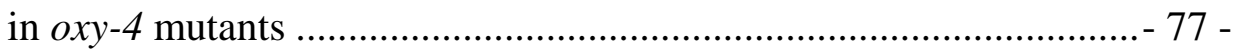

4.5.5 Do Oxy-4 and Ce-lamin act via the same pathway? ........................... 79 -

5 Discussion 82 -

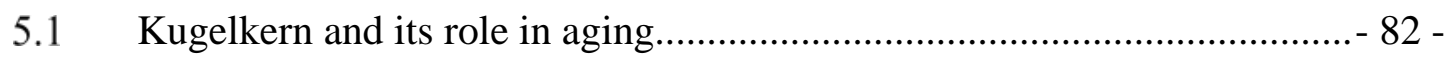

5.1.1 The kuk deficient fly line $\Delta 15$ might still contain recessive mutation(s)- 82 -

5.1.2 Proteolytic cleavage might be mediated by PEST-sequences ............ 83 -

5.2 Narf is involved in aging in Drosophila ................................................... 84 - 
5.2.1 Narf might have an essential role in Fe-S protein maturation ............. 85 -

5.2.2 Lifespan analyses as a tool to study aging......................................... 86 -

5.2.3 Narf overexpression might increase intracellular stress defense mechanisms ........................................................................... 87 -

5.2.4 An altered Narf expression in muscle cells probably leads to redox

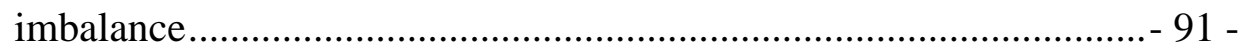

5.2.5 The human mutation might represent a loss-of function allele .......... 94 -

6 Materials and Methods ............................................................................. 95 -

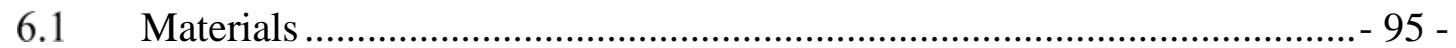

6.1.1 General Buffers ........................................................................ 95 -

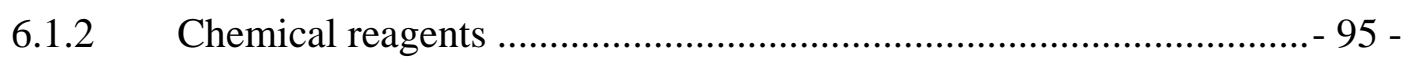

6.1.3 Laboratory services....................................................................... 95 -

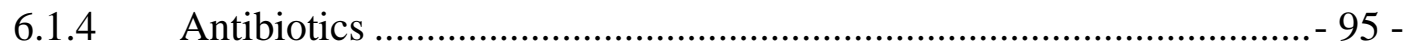

6.1.5 Enzymes and Kits .......................................................................... 96 -

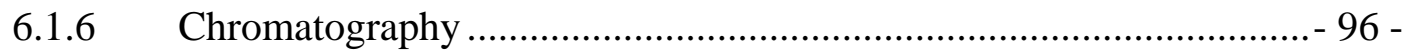

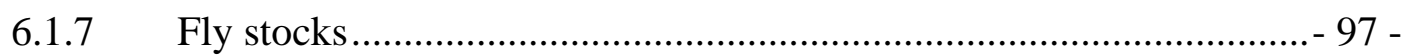

6.1.8 Worm strains and RNAi-vectors used in this study ........................... 98 -

6.1.9 Media for flies .............................................................................. 99 -

6.1.10 Media for C.elegans ….................................................................... 99 -

6.1.11 Bacterial cell lines ........................................................................ 100 -

6.1.12 Media for bacterial culture ............................................................ 100 -

6.1.13 Oligonucleotides......................................................................... 100 -

6.1.14 Plasmid constructs ....................................................................... 103 -

6.1.15 Primary antibodies................................................................. 105 -

6.1.16 Secondary antibodies ................................................................... 106 -

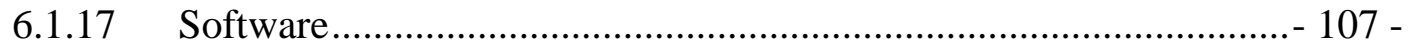

6.1.18 Equipment.................................................................................. 107 -

6.1.19 Other materials .............................................................................. 108 -

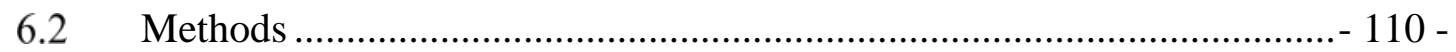

6.2.1 DNA methods ............................................................................... 110 -

6.2.2 RNA methods .......................................................................... 113 -

6.2.3 Biochemical methods ................................................................ 114 -

6.2.4 Antibodygeneration ...................................................................... 117 - 


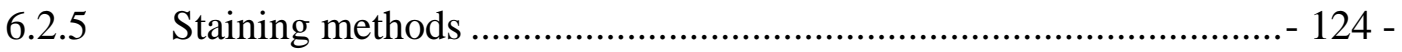

6.2.6 Fly work methods ......................................................................... 127 -

6.2.7 Worm work methods ................................................................ 129 -

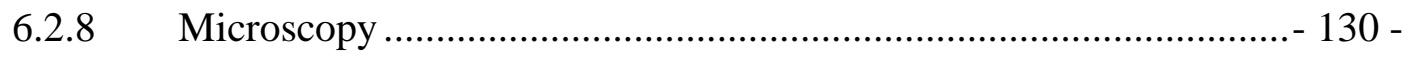

$7 \quad$ References ............................................................................................................... 132 -

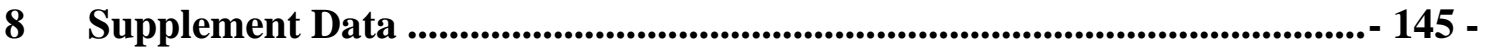

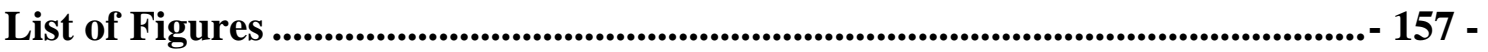

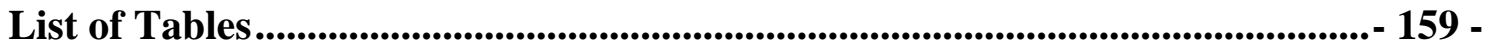

Curriculum Vitae.......................................................... Fehler! Textmarke nicht definiert. 


\section{Acknowledgments}

First of all I would like to thank Prof. Jörg Großhans for giving me the opportunity to do my PhD in his laboratory and to get the chance to carry out a project within the aging field. I am very thankful for all the discussions, his support and his positive thinking during the 4 years of my $\mathrm{PhD}$.

I would like to thank my thesis committee members Prof. Reinhard Schuh and Prof. Harald Herrmann-Lerdon for their suggestions and constructive criticism during our meetings. I am thankful to Prof. Bernd Wollnik for sharing the Narf-project with me.

I would like to thank Prof. Yosef Gruenbaum, who gave me the oppertunity to work in his laboratory and getting the chance to work with C.elegans, another very fascinating model organism. Special thanks go to Ph.D. Noam Zuela, Chayki Charar, Tam Yadid, Jehudith Dorfmann and Perah Spann, who introduced me in to the C.elegans methods and supported me during my stay. I would also like to thank Prof. Henrik Bringmann, as I was allowed to finish my C.elegans project in his laboratory. In addition, I would like to thank the Boehringer Ingelheim Founds for the funding of the C.elegans project in Israel.

Furthermore, I am grateful to all members of the Department of Developmental Biochemistry for the nice working atmosphere and our regular seminars. I would like to thank the TAL lab for performing the RNAseq analysis and their helpful discussions.

A special thank goes to all members of the "fly group". I am happy that I had the chance to work with all of you together. I really enjoyed our friendly and cooperative environment! I am thankful to Dr. Michaela Clever and Dr. Roman Petrovsky for sharing their results, their supportive discussions and ideas during our "monday” meetings. I would like to express thanks to Long Li for his friendship and for helping me to take care of my fly cages at the weekend. I am thankful to Johannes Sattmann for assisting me with the lifespan analyses. Furthermore I would like to show gratitude to Anja Schmidt and Dr. Franziska Winkler, who helped me a lot during my PhD and shared their expertise with me whenever I needed advice. I am also very thankful to Dr. Deqing Kong, Dr. Katja Kriebel, Dr. Maja Gere and Dr. Zhiyi Lv, who were willing to read parts of my first versioned thesis. Thank you for your patience and suggestions!

I would like to thank my family for their support and believe in me. And last but not least I am very grateful to Florian Rest, for his endless support and understanding. 


\title{
Abbrevations
}

\author{
$\% \quad$ percent \\ $\Delta \quad$ deletion \\ ${ }^{\circ} \mathrm{C} \quad$ degree Celsius \\ $\mu \quad$ micro- \\ A ampere \\ aa amino acid (s) \\ $\mathrm{Ab} \quad$ antibody \\ Amp Ampicillin \\ ATP adenosine triphosphate \\ bp base pairs \\ BSA bovine serum albumin \\ C. elegans Caenorhabditis elegans \\ cDNA complementary DNA \\ cm centimeter \\ Co-IP Co-immunoprecipitation \\ $\mathrm{CV} \quad$ column volume (s) \\ d day (s) \\ D.m. Drosophila melanogaster \\ Da Dalton \\ DAPI 4', 6' - Diamidino-2-phenylindole \\ DEPC diethyl pyrocarbonate \\ $\mathrm{ddH}_{2} \mathrm{O}$ double distilled water \\ DNA deoxyribonucleic acid \\ DTT 1,4-dithiothreitol \\ E.coli Escherichia coli \\ EDTA ethylenediaminetetraacetic acid \\ et al. latin phrase for "and others" \\ FG phenylalanine-glycine
}

FRAP fluorescence recovery after photobleaching

FRT flippase recognition target

FTase Farnesyltransferase

FTI farnesyl transferase inhibitor 


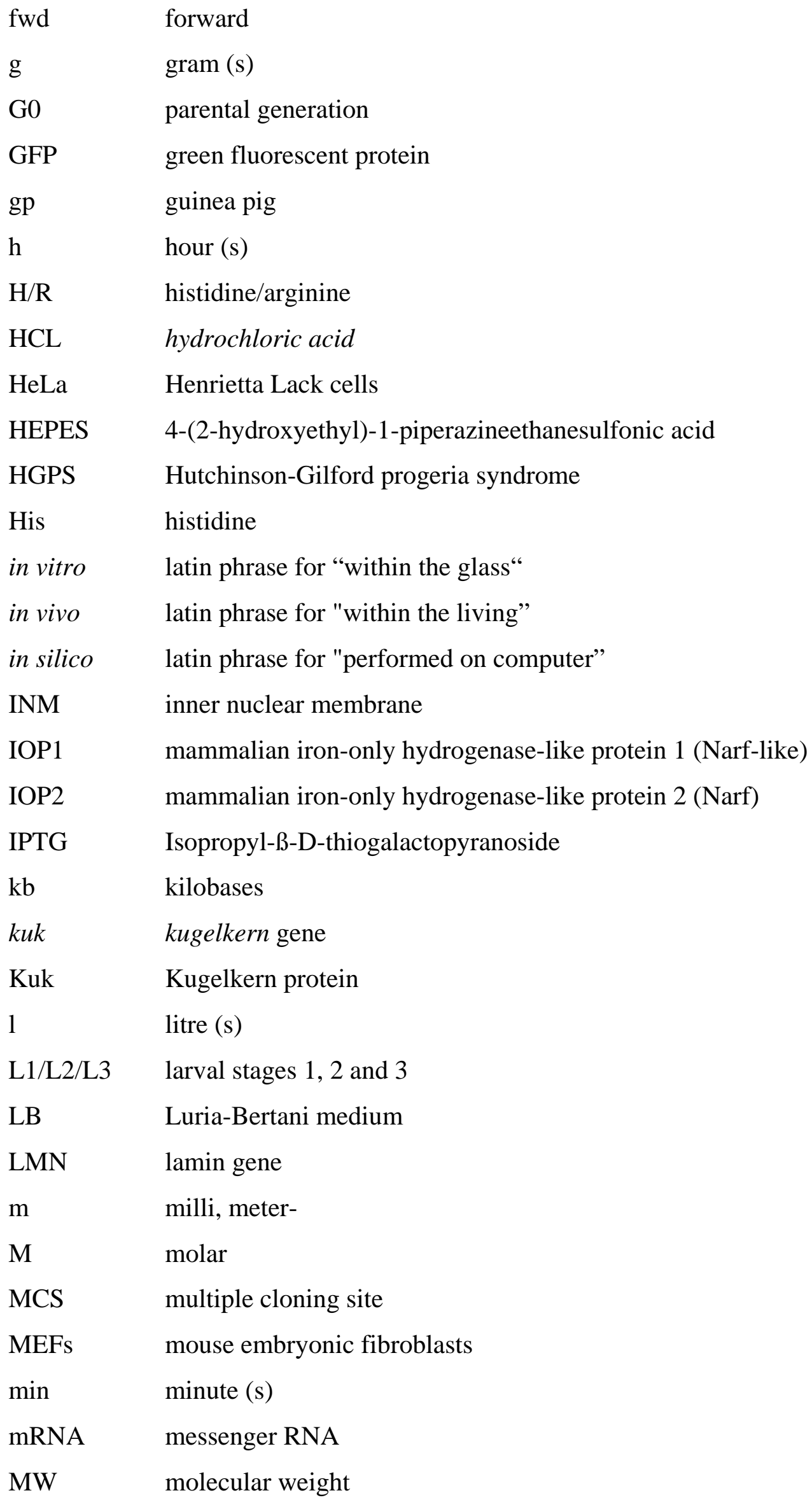




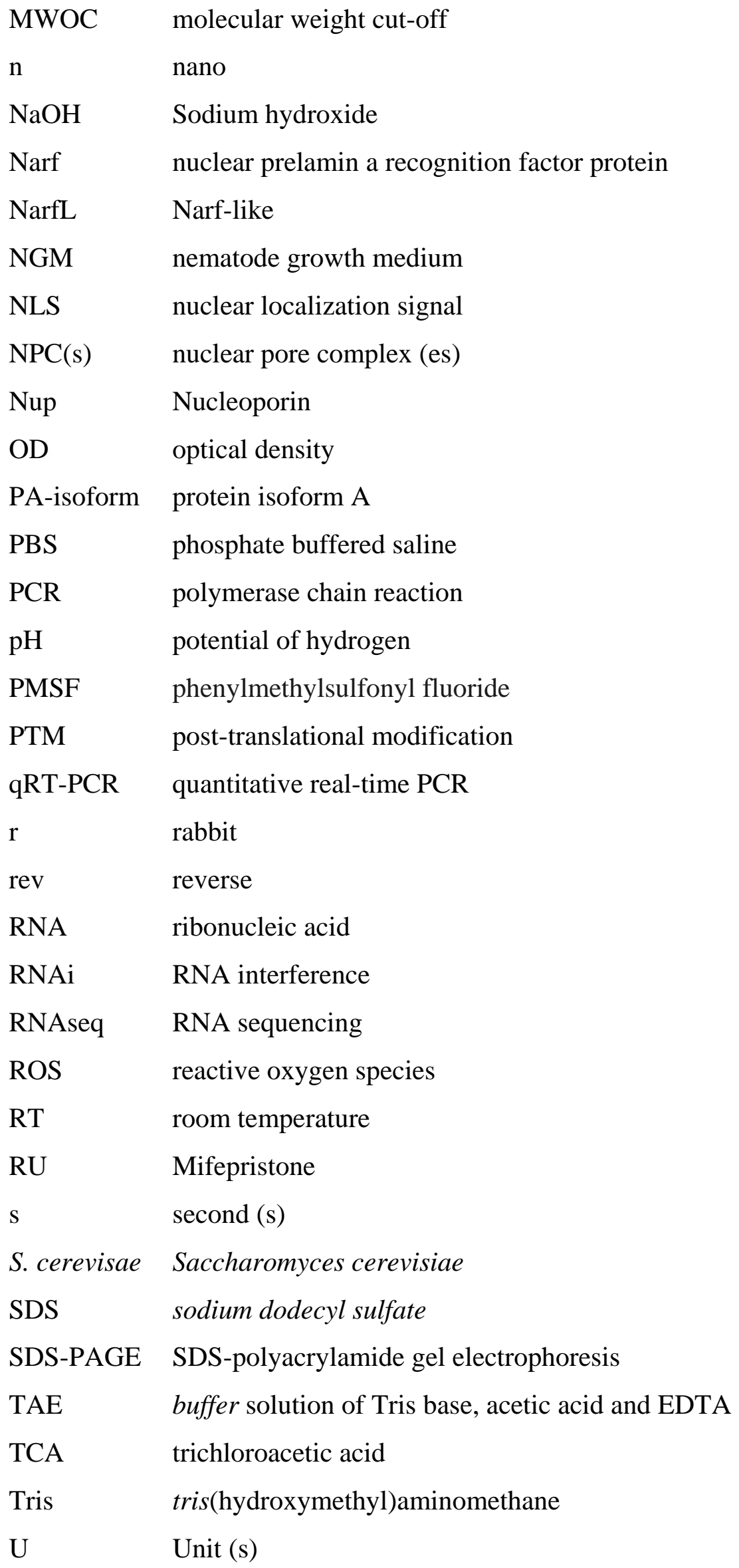




$\begin{array}{ll}\text { UAS } & \text { upstream activating sequence } \\ \text { V } & \text { Volt } \\ \text { v/v } & \text { volume per volume } \\ \text { VDRC } & \text { Vienna Drosophila RNAi Center } \\ \text { w/v } & \text { weight per volume } \\ \text { WT } & \text { wild-type } \\ \text { xg } & \text { g-force } \\ \text { Y2H } & \text { yeast two-hybrid }\end{array}$




\section{Abstract}

Lamins are type $\mathrm{V}$ intermediate filaments and represent the major constituent of the nuclear lamina. Mutations in the laminA/C gene as well as in other nuclear envelope associated proteins lead to a series of human genetic disorders, so-called laminopathies. The most prominent laminopathy is the Hutchinson-Gilford progeria syndrome (HGPS). HGPS patients appear prematurely aged developing aging-related conditions such as osteoporosis, loss of subcutaneous fat and cardiovaskular disease already during childhood. Many of the HGPS patients contain a single point mutation in the lamin $\mathrm{A} / \mathrm{C}$ gene which affects the maturation process and leads to the expression of a permanently farnesylated Prelamin A, called Progerin. Progerin accumulates at the nuclear periphery and leads to a variety of cellular and nuclear defects like a disorganization of heterochromatin structure, an increase in DNA damage and defects in nuclear morphology. Despite the insights in the clinical symptoms and the genetic basis of HGPS and other laminopathies, the molecular and cellular mechanism that leads to accelerated aging is unknown and remains a topic of ongoing research.

The Großhans lab has developed a HGPS model in Drosophila, using lamin Dm0 and Kuk, a nuclear protein which shares structural and functional features to lamins. As farnesylation plays a major role in the disease mechanism, I screened for genetic and biochemical interaction partners of Kuk. I focused on Narf, which has previously been identified as a Lamin binding partner that depends on farnesylation. Furthermore a dominant de novo mutation was recently identified within the human Narf protein causing HGPS related symptoms (Prof. Wollnik, personal communication). Narf is an evolutionary conserved protein with a homologue in Drosophila. As Narf is thought to interact specifically with the farnesylated Prelamin A in humans, I hypothesized that Narf interacts with Lamin Dm0 or Kugelkern in Drosophila. Beside mapping and complementation analysis, nothing was known about the function of Narf in Drosophila. In order to study the cell biological and developmental functions of Narf, I established Drosophila as a model system. I characterized point mutations and generated several tools like an antibody as well as genomic and inducible transgenes. Narf localizes to the nuclear envelope and is essential for development as well as during adulthood. Narf function may rely to its role in Fe-S protein maturation. I demonstrated that Narf is involved in aging-related processes as loss-of function experiments decreased Drosophila`s lifespan whereas constitutively higher expression by multiple genomic copies or UAS overexpression led to a prolongation in lifespan. How Narf might contribute to these processes is not clear yet. RNA sequencing analyses points to a role in oxidative stress response. My data suggest a role of Narf in the regulation of stress defense mechanisms which are required for the maintenance of a cellular redox homeostasis needed for aging. 


\section{Introduction}

\subsection{Nuclear lamins}

The defining property of eukaryotic cells is a nucleus. The nucleus consists of an outer nuclear membrane and an inner nuclear membrane which are fused together at sites of the nuclear pore complexes, allowing the exchange of molecules between the cytoplasm and the nucleoplasm. Metazoan cells additionally contain the nuclear lamina which is underlying the inner nuclear membrane and consist predominantly of lamins which are evolutionary conserved type V intermediate filament proteins. Lamins are required for the maintenance of the nuclear shape and involved in numerous nuclear functions (reviewed in Gruenbaum et. al., 2003, Dechat et al, 2010, Gruenbaum and Foisner 2015, Vidak and Foisner 2016).

Two different types of lamins can be found, namely A- and B-type lamins. A-type lamins are encoded by the LMNA gene in which lamin A and lamin C represent the major isoforms. They are generated by alternative splicing and differ in their C-terminus. A-type lamins are tissue specific expressed. Conversely, B-type lamins are constitutively expressed and are essential for developmental processes. B-type lamins, comprising lamin B1 and B2, are encoded by two distinct genes, namely LMNB1 and LMNB2 (Eckersley-Maslin et al., 2013, Dechat et al., 2010).

Nuclear lamins share a conserved polypeptide structure which comprises a variable head domain, a $\alpha$-helical coiled-coil rod domain and a globular tail domain including an Ig-fold motif, the nuclear localization signal (NLS) and the CaaX motif (Stuurman et al., 1998) (Figure 1 A). B-type lamins and lamin A, but not lamin C, originate from premature lamins and contain a CaaX motif (C- cysteine residue, aa - aliphatic residues, $\mathrm{X}$ - any amino acid) (Liu et al., 2006, Sinensky et al., 1994) (Figure 1 B-C). During lamin maturation the cysteine within the CaaX-motif is farnesylated which is mediated by a farnesyltransferase (FTase). The next processing step includes the cleavage of the last three carboxyl terminal aminoacids (aaX) by the proteases FACE1/ZMSPTE24 or FACE2/Rce1. Finally, a carboxymethylation of the farnesylated cysteine takes place which is mediated by the isoprenyl-cysteinecarboxy-methyltransferase (ICMT). B-type lamins are not further processed and stay farnesylated. Lamin A maturation is completed by a further cleavage of the last 15 carboxy terminal aminoacids, which lead to a removal of the farnesylated and carboxymethylated 
cysteine by FACE1/ZMPSTE24 (Pendas et al 2002, Rusinol and Sinensky 2006, Young et al 2005) (Figure 1 B).

The farnesylated and carboxymethylated CaaX- motif mediates the association of lamins with the inner nuclear membrane (INM). B-type lamins keep the CaaX- motif after processing which lead to a predominant localization to the nuclear envelope. A-type lamins instead lack the farnesyl group in their matured form, which causes a localization to the nuclear envelope as well as to the nucleoplasm (Dechat et al., 2010, Kolb et al., 2011, Moir et al., 2000).

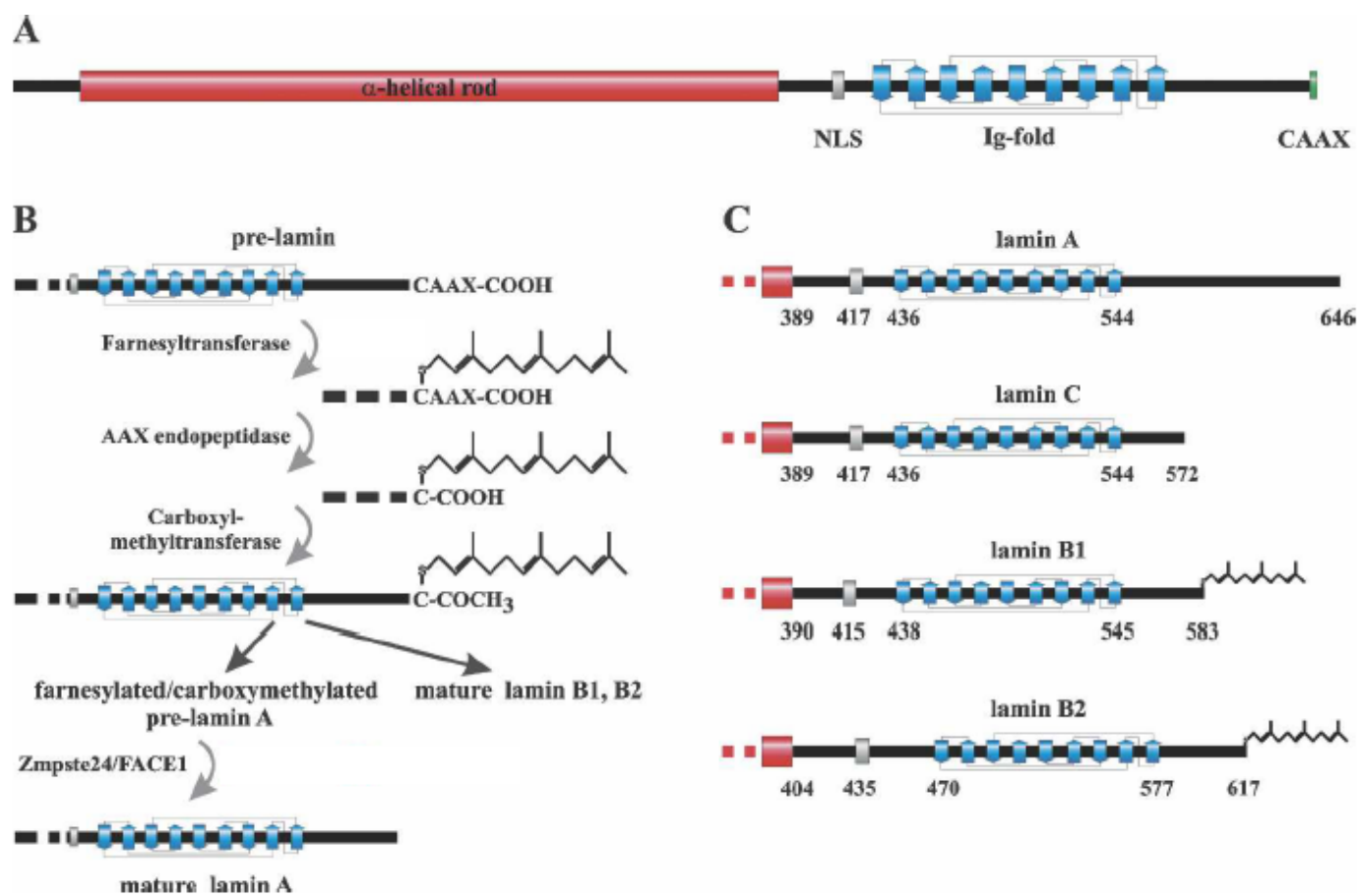

Figure 1: Nuclear lamins share a conserved structure

(A) Illustration of the lamin A structure. Lamins consist of a variable head domain (not shown), $\alpha$ helical coiled-coil rod domain (red) and a globular tail domain (blue), including the nuclear localization signal (NLS, grey) and the CaaX-motif (green). (B) Overview of the processing steps of the premature lamins A, B1 and B2. The processing includes three major steps: first the farnesylation of the cysteine within the CaaX-motif by a farnesyltransferase (FTase), second the cleavage of the last three carboxyl terminal amino acids (aaX) by an endopeptidase (FACE1/ZMSPTE24 or FACE2/Rce1) and third the carboxymethylation of the farnesylated cysteine by an isoprenyl-cysteine-carboxy-methyltransferase (ICMT). B-type lamins are not further processed and stay farnesylated. Lamin A maturation is completed by a cleavage step of the last 15 carboxy terminal amino acids, including the farnesylated and carboxymethylated cysteine by FACE1/ZMPSTE24. (C) Overview of the C-terminal differences in matured lamins. Mature lamin A and lamin C do not possess a farnesylation motif at their C-terminal end. The reasons are the cleavage of farnesylation for lamin A during maturation and 
the lack of a CaaX motif for lamin C. However, B-type lamins stay farnesylated and carboxymethylated (modified to Dechat et al., 2008).

\subsection{Lamin A mutations and Huntchinson-Gilford progeria syndrome}

A series of human genetic disorders, so-called laminopathies are caused by mutations in genes coding for proteins of the nuclear lamina, including lamin A/C. More than 500 mutations are reported until now, which give rise to striated muscles diseases, adipose tissue diseases, periphereal nerve diseases and accelarating aging disorders (reviewed in Broers et al., 2006). The most prominent laminopathy is the Hutchinson-Gilford progeria syndrome (HGPS), which affects 1 in 4-8 million newborns (Progeria Research Foundation). Patients with HGPS appear prematurely aged and develop aging-related conditions already during childhood.

Most of the HGPS patients contain a single point mutation within exon 11 (1824C>T, p.G608G). This mutation causes the activation of a cryptic splice site which lead to an internal deletion of 50 amino acids and by this to a disruption of the premature lamin $\mathrm{A}$ processing. The consequence is the generation of a mutant Prelamin A, called Progerin. Progerin is permanently farnesylated, in contrast to normal Lamin A, as the internal deletion also affects the FACE1/ZMPSTE24 cleavage site (De Sandre-Giovannoli et. al., 2003, Eriksson et al., 2003, Pollex and Hegele 2004, Moulson et. al., 2007). Progerin is accumulating at the nuclear periphery as a consequence to its permanent farnesylation, which mediates the association to the inner nuclear membrane. This leads to severe cellular defects like disorganized heterochromatin, increased DNA damage and defects in nuclear morphology (Dechat et al., 2007, Kitten and Nigg 1991, Krohne 1998, Capell et al., 2005, Gonzalez-Suarez et. al., 2009, Scadaffi et al., 2005). Progerin expression can be also found in physiologically aged humans, suggesting that Progerin contributes to normal aging, too (McClintock et al., 2007). Currently, clinical trials with HGPS patients have been ongoing for a few years with inhibitors of the farnesyl transferase (Young et al., 2006, Gordon et al., 2013, Young et al., 2013, Ullrich et al., 2013). Less clear than the clinical symptoms and the genetic basis of HGPS and other laminopathies, is the molecular and cellular mechanism that leads to accelerated aging. Several models, such as reduced mechanical stability, dysfunctional nuclear transport, impaired chromatin organization and increased DNA 
damage have been proposed, but none of these models provides a full explanation (reviewed in Gosh and Zhou 2014, Gruenbaum and Foisner 2015).

\subsection{Drosophila as a model to study aging}

Aging processes are a field of extensive research, whereby Drosophila is used as a model system to study lamins in general but also to mimic distinct lamin mutations causing human diseases (e.g. Schulze et al., 2009, Munoz-Alarcon et al., 2007, Dialynas et al., 2015). Compared to humans, where two types of lamins can be found, most of the invertebrates possess only one B-type lamin. However, Drosophila represents an exception among invertebrates as two lamins, lamin Dm0 and lamin C are expressed. Lamin Dm0 is grouped to B-type lamins by the presence of a CaaX-motif and its ubiquitous expression during development. Lamin $\mathrm{C}$ is classified as an A-type lamin, lacking a CaaX box and being expressed in a developmentally regulated manner (Erber et al., 1999, Gruenbaum et al., 1988, Bossie and Sanders, 1993, Riemer et al., 1995).

Drosophila contains a second farnesylated nuclear protein next to the farnesylated B-type lamin Dm0, which is named Kugelkern (Kuk) due to its function in nuclear morphogenesis (Brandt et al., 2006). The Grosshans lab has established a Drosophila HGPS model (Brandt et al., 2006, 2008), in which this mechanism can be studied in a genetically tractable organisms. Kuk is localized to the nuclear side of inner nuclear membrane and causes ruffled and overlobulated nuclei, a reduction in heterochromatin and shortened mean lifespan of flies similar to lamin Dm0 (Brandt et al., 2008). Overexpression of the farnesylated Kuk in Drosophila and Lamin proteins in humans induce similar aging related phenotypes (Figure 2). Thus, it might be beneficial to study the function and activity of Kuk in order to enhance the understanding of premature aging in HGPS. 
Lamin A

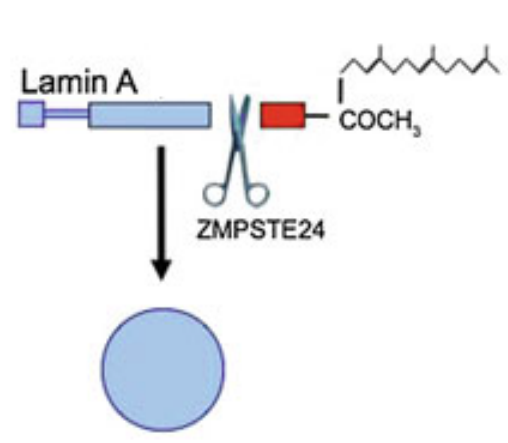

HGPS

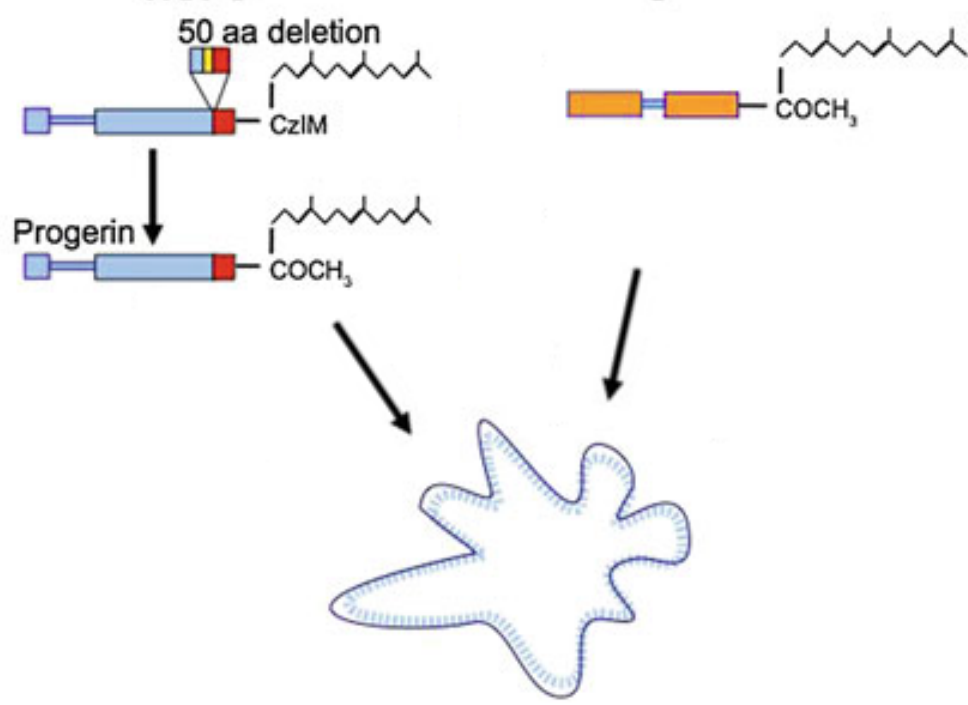

Figure 2: The effect of farnesylated nuclear proteins on nuclear morphology

The normal processing of Pre-lamin A includes the removal of the farnesylated cysteine within the CaaX-motif by a FACE1/ZMPSTE24 mediated cleavage of the last 15 carboxy terminal amino acids (red). This last processing step is disrupted in HGPS patients due to an internal deletion of 50 aminoacids (blue-yellow-red box) which is caused by a single point mutation (yellow). This leads to a mutant Pre-lamin A, called Progerin. Expression of this mutant protein leads to accumulation at the inner nuclear membrane and causes cellular defects like disorganized heterochromatin, increased DNA damage and defects in nuclear morphology. Same effects have been observed in Drosophila melanogaster overexpressing Kugelkern (Kuk), a nuclear protein that do not belong to lamins. Kuk shares structural similarities like a putative coiled-coil region (blue), a nuclear localization signal (not shown) and a farnesylated CaaX-motif. Not conserved features are shown in orange (modified to Brandt and Vilcinskas, 2013).

\subsection{Narf - a new progeria gene?}

The group of Prof Bernd Wollnik (Institute for Human Genetics, Göttingen) recently identified a dominant de novo mutation in the protein Narf in a Turkish patient with symptoms related to HGPS, mimicking clinical features (e.g. reduced subcutaneous fat, mild osteoporosis) and nuclear morphology (e.g. ruffled nuclei) (personal communication Prof. Wollnik). Initially Narf was identified as a binding partner of farnesylated Prelamin A. Narf binds the carboxyl-terminal tail of Prelamin A dependent on farnesylation (Barton and Worman, 1999). The authors proposed that Narf could be a co-factor of ZMPSTE and might be important for Lamin A processing or might act as an anchor for farnesylated Prelamin A to organize nuclear proteins. 


\subsection{Narf like genes have a common ancestor and play a role in Fe-S biogenesis}

Narf-like genes are quite conserved as they share a common ancestor with [Fe] -only hydrogenases. Due to this they are also known as eukaryotic hydrogenase like proteins. Hydrogenases in general are enzymes in anaerobic organisms, which are needed for anaerobic respiration as they are catalyzing the oxidation of molecular hydrogen: $2 \mathrm{H}^{+}+2 \mathrm{e}^{-} \rightleftharpoons \mathrm{H}_{2}$ (reviewed in Nicolet et al., 2002).

However, Narf-like genes do not produce hydrogen anymore and it is supposed that they developed new functions during development (Hackstein, 2005). One of the best characterized Narf-like genes is the yeast homologue nar1. Nar1 belongs to the group of FeS-proteins and is essentially required for the maturation of cytosolic and nuclear Fe-S proteins (Balk et. al., 2004, 2005). Fe-S protein biogenesis is a quite conserved mechanism and Fe-S proteins are involved in a variety of processes including oxygen sensing, electron transport, Fe-S protein maturation and transcriptional regulation (Nakamura et al., 2013, reviewed in Johnson et al., 2005).

In mammalian, the two orthologous Narf (nuclear prelamin $\underline{A}$ recognition factor) or IOP2 (iron-only hydrogenase-like protein 2) and Narf-like or IOP1 are known. It was shown in HeLa cells and MEFs (mouse embryonic fibroblasts) that IOP1 is involved in biogenesis of mammalian cytosolic Fe-S protein as a knockdown situation is affecting the activity of different cytosolic Fe-S proteins but has no effect of tested mitochondrial Fe-S proteins or non Fe-S proteins (Song et al., 2009; Song and Lee, 2008, 2011). The described effects were shown to be specific for IOP1 as a knockdown for IOP2/Narf did not affect Fe-S protein maturation (Song and Lee, 2008). The effect of an IOP1 depletion in mice leads to lethality during early embryogenesis as well as in adults if the knock-out was performed in an inducible way (Song and Lee, 2011). On the molecular level it was described that IOP1 depletion in MEFs selectively activates intracellular signaling pathways like caspase dependent apoptosis and stress kinase p38 mediated autophagy (Song and Lee, 2011). Comparable with yeast, it was described in Hela cells that a knockdown of IOP1 leads to a global decrease in protein synthesis which finally leads to a growth impairment and a decreased cell viability (Song and Lee, 2008). 


\subsection{Role of Narf-like genes in sensing oxygen}

Loss of function studies performed for Narf-like genes revealed a sensitivity against oxygen. Growth defects during development at high concentrations as well as under normoxic conditions were observed for example in C. elegans (oxy-4), S. cerevisiae (Nar1) and A. thaliana (Gollum) (Cavazza et al., 2008; Fujii et al., 2009; Mondy et al., 2014).

In yeast, Nar1 mutant cells exhibited a higher level of reactive oxygen species (ROS) even in non-stressed situations, which is even more increased at high concentrations of oxygen. One explanation for this finding was given by the observation that a loss of Nar1 leads to a decrease of mitochondrial SOD2 mRNA level, one of the superoxide detoxifying genes and critically required for efficient oxidative stress protection in yeast (Steinman, 1980; Sturtz et al., 2001; Zhao et al., 2014). It was supposed that a loss of Nar1 causes a defect in the maturation of several Fe-S protein, which consequently damages mitochondria and leads to a decrease in SOD2 (Zhao et al., 2014). In plant and yeast growth arrest can be rescued with hypoxic conditions (Cavazza et al., 2008; Fujii et al., 2009), suggesting that Nar1 and Gollum might be involved within an oxygen sensitive step in Fe-S protein maturation or are not essential at low oxygen concentrations. However, complementation of the plant Gollum gene within in a Nar1 yeast mutant strain was not achieved, meaning that they either functions in different pathway or interact with different interaction partner (Cavazza et al., 2008).

In mammalian cells an additional oxygen response pathway was linked to IOP1. It was shown that a knockdown of IOP1 (Narf-like) but not of IOP2 (Narf) results in an up regulation of HIF $1 \alpha$ (hypoxia inducible factor $1 \alpha$ ), which is a key transcription factor under hypoxic conditions (Huang et al., 2007). The HIF transcription factor functions as a heterodimer and consist of $\alpha$ - and $\beta$-subunits. In case of hypoxia, the subunits are dimerizing and translocate to the nucleus to allow gene expression (reviewed in Semenza, 1999). Under normoxic conditions, proline residues of the HIF1 $\alpha$ subunit are permanently hydroxylated by a family of PHDs (proline hydroxylase domain-containing protein), which marks HIF1a and finally leads to its degradation (Bruick and McKnight, 2001; Ivan et al., 2001; Jaakkola et al., 2001; Salceda and Caro, 1997). A Yeast-two-hybrid assay showed that IOP1 and PHD2 are direct interaction partners, showing a function of IOP1 under normoxic and hypoxic conditions (Huang et al., 2007). A shRNA approach of IOP1 in hyperoxia resistant HeLa cells caused increased oxygen sensitivity and identified IOP1 to be active also under 
hyperoxic conditions. The increase in oxygen resistance was achieved by the up regulation of IOP1 mRNA and protein levels (Corbin et al., 2015). Fe-S proteins are sensitive against ROS and oxygen as the iron is easiliy oxidizied (reviewed in Johnson et al., 2005). It was shown that IOP1 was able to protect the cytosolic and nuclear Fe-S proteins in presence of high oxygen and thereby overcome the problem of oxidative stress (Corbin et al., 2015).

\subsection{Role of Narf-like genes in aging}

A link between Fe-S protein maturation and genome stability was made in 2012. It was shown that depletion of IOP1 in HEK293 cells leads to a defective maturation of XPD, which is a component of the transcription factor complex TFIIH and involved in nucleotide excision repair (Gari et al., 2012, Rudolf et al., 2006, Stehling et al., 2012). Several other nuclear Fe-S proteins are known to play a role in DNA repair, replication and telomere length. It is likely that a defect in Fe-S protein maturation affects multiple DNA repair pathways which finally leads to genomic integrity a known characteristic of aging (Gari et al., 2012, Stehling et al., 2012).

Lifespan analyses in C.elegans and yeast, demonstrated the role of Narf-like genes in aging (Fujii et al., 2009, Zhao et al., 2014). The reasons for a decrease in lifespan were made by an increased sensitivity to oxygen. It is a well-known fact that oxygen and the resulting reactive oxygen species (ROS) are involved in the damage of DNA, proteins and lipids. By this oxidative stress leads to accelerated aging. An interesting link between vertebrates and invertebrates was given by the observation that the mutant allele of the C.elegans ortholog Oxy-4 possesses an amino acid exchange at exactly the same position like it was described for human Narf/IOP2 (Fujii et al., 2009, personal communication Prof. Wollnik). Besides, of being a binding partner of farnesylated Prelamin A nothing is known for Narf/IOP2. As Narf and IOP1 differ in protein localization and are not able to complement each other, it is supposed that they do not have the same functions, hypothesizing a direct role of Narf/IOP2 in aging (Barton and Worman, 1999, Song and Lee, 2008, 2011). However, the molecular mechanism of how Narf-like genes affect the lifespan are not well characterized yet. 


\section{Aim of the study}

Overexpression of the farnesylated Kuk induces cellular defects that lead to aging related phenotypes. One aim of this thesis was the identification of Kuk binding partners or complexes and to determine the role of farnesylation. Furthermore, CG17683 the Drosophila homologue of the human Narf was analyzed. As Narf is an already identified Lamin binding partner and dependent on farnesylation it was hypothesized that CG17683 specifically interacts with Lamin Dm0 or Kugelkern in Drosophila. Beside mapping and complementation analysis, the function of Narf in Drosophila has not been studied. Due to this, the function of Narf during development, the connection of Narf in aging in Drosophila and the effect of Narf to the farnesylation or subcellular distribution of Kugelkern and/or Lamin Dm0 was analyzed. 


\section{Results}

\subsection{Identification of novel Kuk interaction partners}

The Drosophila protein Kugelkern (Kuk) is the only known nuclear envelope protein, excluding lamins, which also contains a CaaX-motif enabling farnesylation. It was shown that Kuk shares further structural similarities with lamins, like a nuclear-localization signal (NLS) and a putative coiled-coil domain. Kuk is characterized through an early zygotic gene-expression and is required for nuclear elongation. An overexpression of Kuk causes overlobulated nuclei and is connected to accelerated aging phenotypes in adult flies (Brandt et al., 2006, 2008). It is hypothesized that these effects are partially dependent on Kuk-farnesylation, suggesting the mediation of a distinct protein-complex formation. The aim of this study was the identification of farnesylation dependent interaction partners of Kuk and to determine the role of farnesylation in protein complex formation.

\subsubsection{Characterization of $\Delta 15$ a kuk deficient fly line}

To study Kugelkern function a deletion mutant was generated by the Großhans laboratory. Therefore, $\mathrm{a} \mathrm{w}^{+}$marked transposon was mobilized and a lethal point mutation within CG5169 (GckIII) was complemented with a genomic rescue construct (Brandt et al., 2006). However, the deletion breakpoints were not fully characterized yet. To narrow down the breakpoints, genomic DNA of kuk deficient flies $(\Delta 15)$ was isolated and PCR reactions comprising the kuk neighboring regions were performed. A long range PCR, using the oligonucleotides MK26 and MK29, revealed a size difference of the wild type PCR product compared to the mutant gene locus. Sequencing analyses confirmed that the depletion of the $k u k$ deficient line comprises a gene region of $5.5 \mathrm{~kb}$ with an additional insertion of $465 \mathrm{bp}$ from the originally inserted P-element vector. An illustration of the described characterization is shown in Figure 3. The sequencing results of the deletions breakpoints are shown in Supplement Figure 1. 

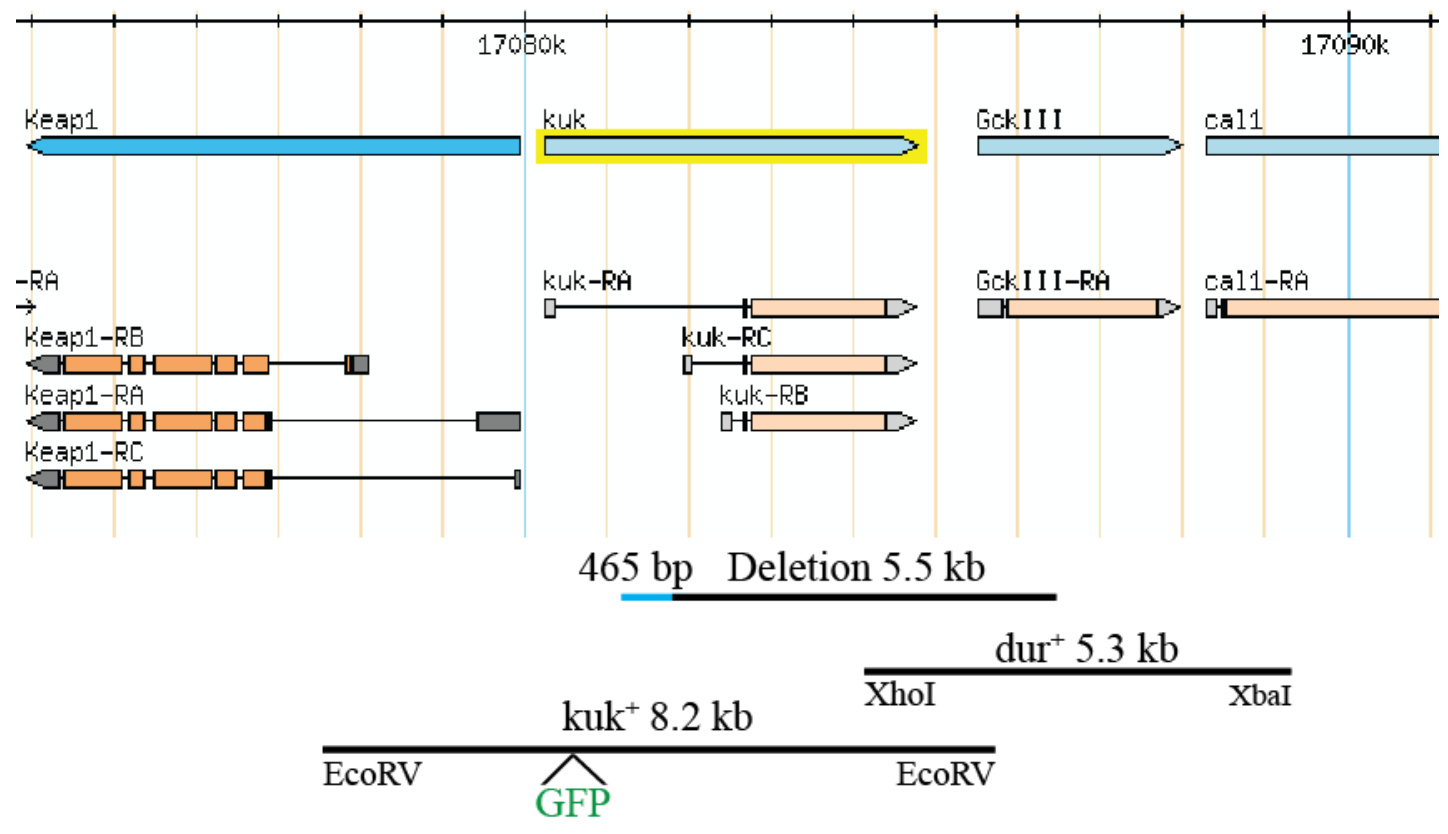

Figure 3: The deletion of a kuk deficient line comprise $5.5 \mathrm{~kb}$ within the genomic locus

The genomic breakpoints of the deletions were analyzed by PCR, including the neighboring genes of kuk. Final characterization was done by a long range PCR (MK26/MK29) followed by a subsequent sequencing analysis. The sequencing results revealed a depletion of $\sim 5.5 \mathrm{~kb}$ within the genomic locus of kuk and GckIII (dur). Furthermore, an insertion of 465 bp due to initially performed transposon remobilization was found. Furthermore the rescue constructs of dur (GckIII) and kuk are depicted. Scheme of the genomic region was modified according to http://flybase.org/reports/FBgn0038476.

\subsubsection{Nuclear elongation is missing in $\Delta 15$ embryos}

Original Kugelkern loss of function data were produced using a heterozygous $\Delta 15$ fly strain, which was kept over deficiency, as recessive lethal mutations existed in combination with the kuk depletion (Brandt et al., 2006). Meanwhile, the chromosome was cleaned, secondary lethal effects were removed and a homozygous $\Delta 15$ fly strain was established (M. Clever). Western blot analysis of mutant embryo extract revealed that the kuk deficient fly line $\Delta 15$ represents a protein null allele (Figure $4 \mathrm{~A}$ ). To verify if homozygous $\Delta 15$ flies still show the described kuk phenotype of round shaped nuclei at the end of cellularization, an embryo immunostaining was performed (Figure 4 B). A staining against lamin Dm0 was used as a marker for the nuclear envelope, whereas Slam indicates the cellularization progress. In wild type embryos a progressive elongation of round shaped nuclei can be observed over time starting with the furrow invagination. Compared to this, nuclei in kuk depleted embryos stayed rounded and formed multiple nuclei layers. As the 
aim of this study was the identification of farnesylation dependent Kuk interaction-partners, I further examined the nuclear morphology phenotype of a non-farnesylable mutant (KukC567S) within a $\Delta 15$ background. This transgene encodes for a cysteine to serine exchange (C567S) within the CaaX-motif of the native kuk. It is shown that the expression level of the mutant protein and its wild type homolog are comparable (Figure $4 \mathrm{~A}$ ). The missing farnesylation was confirmed in a SDS-Page, due to the smaller molecular mass of KukC567S and the induced band shift. Thus, the mutant Kuk protein is not sufficient to rescue the $\Delta 15$ phenotype. Previous studies showed that expression of a non-farnesylated KukCS protein in wild-type flies caused a clear localization to the nuclear envelope. This was reasoned by homo-dimerization with farnesylated, endogenous Kuk-protein (Brandt et al., 2008). In a background without endogenous Kuk ( $\Delta 15)$, the GFP-tagged KukC567S is mislocated to the nucleoplasma compared to the typical nuclear rim localization of the GFP tagged wild type Kuk (Figure $4 \mathrm{C}$ ).

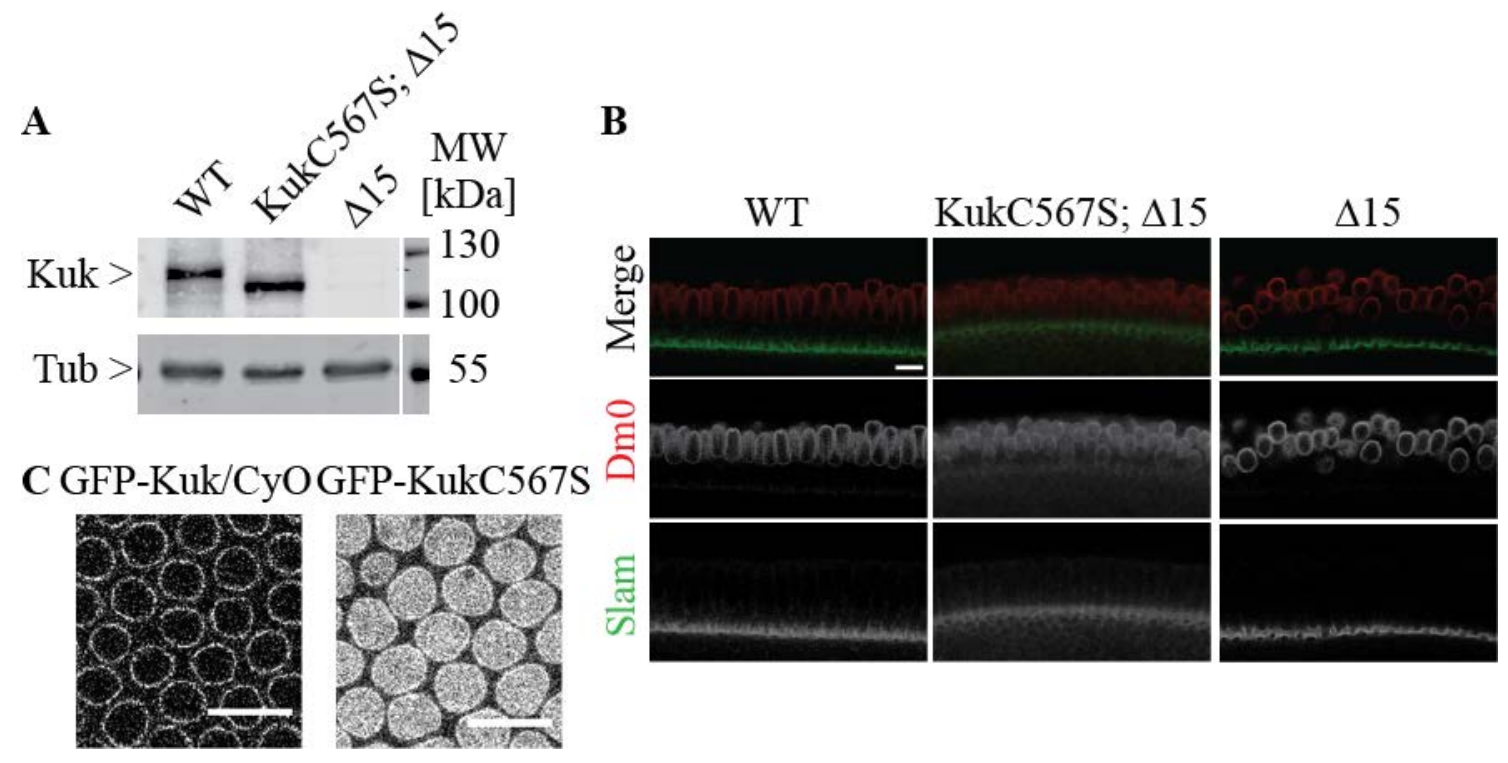

Figure 4: The $k u k$ deficient line $\Delta 15$ represents a protein null allele

(A) Western blot analysis of wild-type and $\Delta 15$ embryo extracts revealed that $\Delta 15$ is a Kuk-protein null allele. Tubulin was used as loading control. Embryo extract of KukC567S samples showed a band shift due to the loss of farnesylation. Western blot analysis was performed with the extract of 20 embryos. (B) Side view of an embryo immunofluorescence staining at the end of cellularization stained for lamin Dm0 and Slam. Wild type embryos showed the nuclear elongation phenotype, whereas $\Delta 15$ and KukC567S; $\Delta 15$ embryos remained rounded. Scale bar: $10 \mu \mathrm{m}(\mathrm{C})$ Top view from live-imaging of a genomic GFP-Kuk wild type allele and a nonfarnesylable GFP-KukC567S mutant. The farnesylated wild type allele localized to the nuclear envelope, whereas the mutant protein is found predominantly within the nucleoplasm. Both genomic transgenes were expressed in a kuk deficient background $(\Delta 15)$. Scale bar: $10 \mu \mathrm{m}$. 


\subsubsection{Gene expression is altered in kuk-depleted embryos}

To validate if gene expression is affected by the loss of $k u k$, RNA sequencing analyses were performed. kuk-depleted embryos showed a change in their genome-wide expression profile during cellularization compared to wild type embryos (Brandt et al., 2006). In this transcriptom analyses embryos from stage 5 to 7 were examined and a down regulation of early zygotic genes like slam, nullo and frühstart have been observed. In this study $\Delta 15$ and wild type control embryos were collected after 6-8 hours referring to stage 11 and 12. 670 genes were differentially expressed in kuk-depleted embryos. The top 50 upand down-regulated candidates based on their log2fold were selected. These 100 candidates were further sorted based on the p-value and the base Mean ( $\mathrm{p}$-value $\geq 1.01 * 10^{-3}$, base Mean $<17.65$ for up regulated and $<46.39$ for down regulated genes). By this it was possible to narrow down 50 candidate genes (Supplement Table: 1). A functional classification was performed using the Panther software (www.pantherdb.org). With this 20 genes were successfully grouped, whereas 30 genes were not further classified (Figure 5). One of the unclassified genes is $k u k$, which is strongly down regulated in $\Delta 15$ embryos and was used as control. Compared to the previously performed analysis by Brandt et al., an enrichment for genes containing a catalytic activity (group 3) was found. Out of these, 5 candidates (10\%) are described for having an oxidoreductase activity (CG18547, CG3397, Cyp9b1, CG7675 and Cyp6d2). A change of gene expression within these proteins is indicative for an oxidative stress situation upon kuk-depletion. Furthermore, the anti-oxidative factor GstD1 was up regulated ( $\log _{2}$ fold: +6.02 , group1). Additionally, methuselah-like 8 ( $\log _{2}$ fold: -11.25 ) and dawdle (log $\log _{2}$ fold: +5.12 ) were identified. Both proteins are linked to aging in Drosophila, suggesting a connection to genes involved in oxidative stress response. 
Panther GO-Slim - Molecular Function

Total \# Genes: 20 \# Function Hits: 32

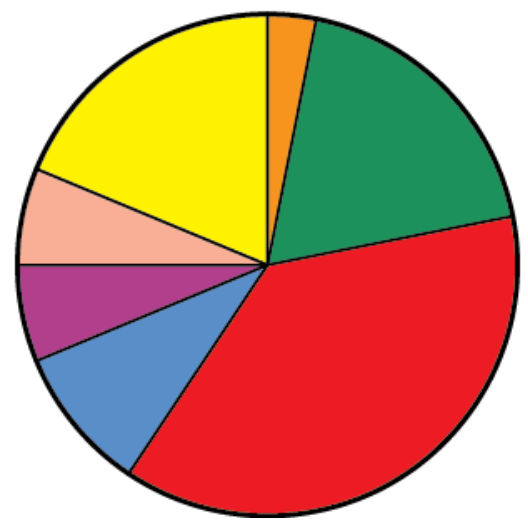

$\square$ Group 1: antioxidant activity (1)

$\square$ Group 2: binding (6)

$\square$ Group 3: catalytic activity (12)

$\square$ Group 4: reporter activity (3)

$\square$ Group 5: signal transducer activity (2)

$\square$ Group 6: structural molecule activity (2)

$\square$ Group 7: transporter activity (6)

Figure 5: Differentially expressed genes in kuk-depleted embryos

Enrichment analysis based on the molecular function was performed using the PANTHER tool as a classification system. Seven groups have been described to contain at least one of the final 50 candidates. Unclassified genes are not considered in this schematic illustration and are referred as group "0" (Supplement Table: 1).

\subsubsection{Survival rate is affected in kuk-depleted animals}

In order to analyze if the observed gene expression changes for genes involved in stress response are associated with an accelerated aging effect, lifespan assays were performed (Figure 6 A). It was found that a loss of Kuk is only slightly affecting lifespan. However, the introduction of non-farnesylable mutant in a kuk deficient background decreased lifespan. KukC567S flies showed a 50\% mortality at day 32, compared to wild type and $\Delta 15$ flies with a $50 \%$ mortality at day 42 . The observed decrease in lifespan by the expression of a mutant Kuk allele might be due to the observed Kuk miss-localization (Figure $4 \mathrm{C}$ ). To analyze if gene expression changes are associated to a developmental phenotype in kugelkern deficient animals, survival rate analyses were performed. $\Delta 15$ animals showed a strong effect on embryonic viability as only approximately $25 \%$ of the larvae hatched (Figure 6 B). The introduction of a transgenic non-farnesylable Kuk protein (KukC567S) in kuk-depleted flies did not rescue the observed phenotype, even if survival during adult stage was increased up to $38 \%$ (Figure 6 B). However, survival rate analyses of animals which are only homozygous for a kuk depletion ( $\Delta 15 /$ Def) did not show an effect on viability compared to wild type control animals, suggesting that the observed lethality in homozygous $\Delta 15$ embryos is kuk independent. 
A

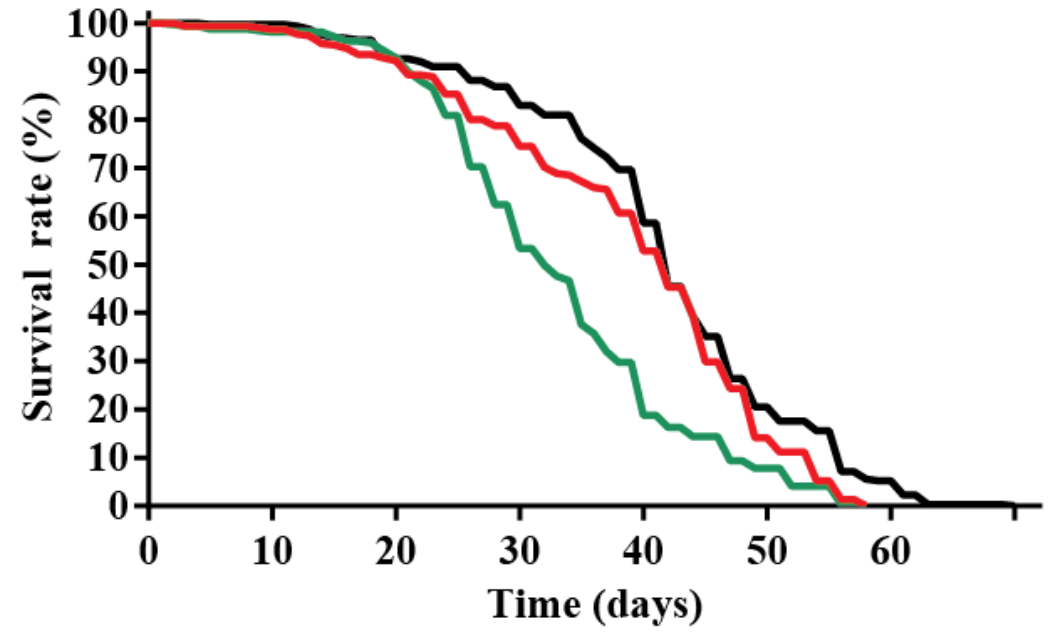

- WT $(\mathrm{N}=315,4$ experiments $)$

- $\Delta 15(\mathrm{~N}=308,4$ experiments $)$

— KukC567S; $\Delta 15(\mathrm{~N}=319,4$ experiments $)$

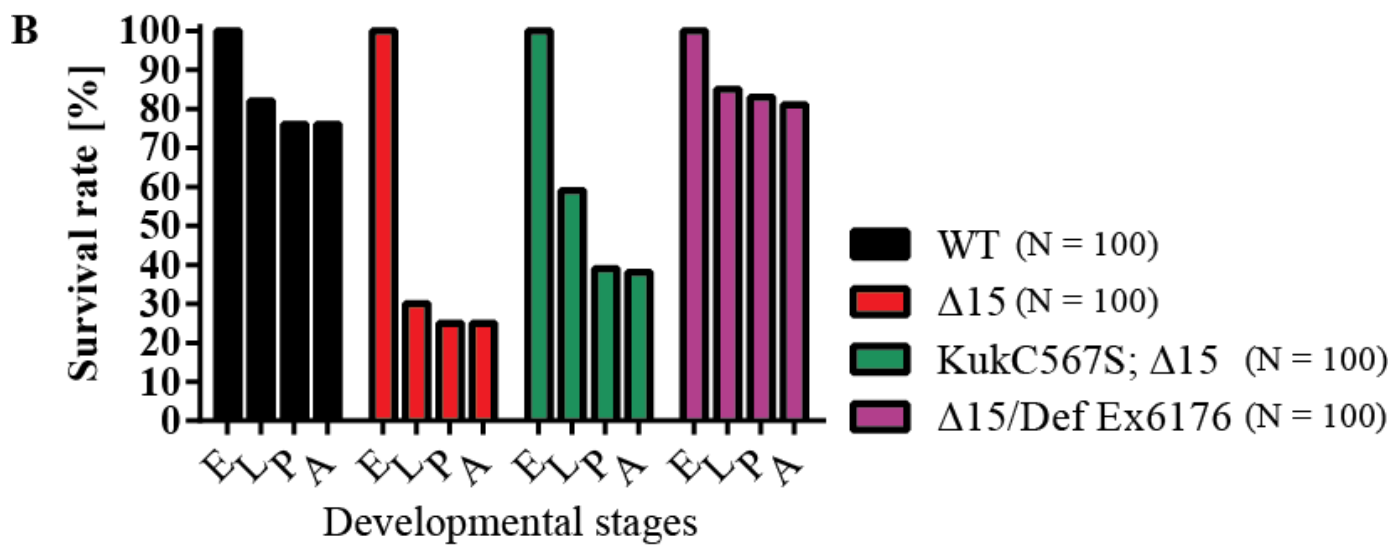

Figure 6: kuk-depleted animals show a decreased survival rate and lifespan

(A) Lifespan analyses showed that kugelkern depletion slightly affected viability during adulthood (log-rank test: WT vs. $\Delta 15 \mathrm{p}=0.0012$ ). The KukC567S expression in a $\Delta 15$ background instead caused a more severe shortening in lifespan (log-rank test: WT vs. KukC567S; $\Delta 15 \mathrm{p}<1.0 * 10^{-10} ; \Delta 15$ vs. KukC567S; $\Delta 15$ $\mathrm{p}<1.0 * 10^{-10}$ ). Statistical analyses were performed by OASIS. (B) Survival rate analyses confirmed a high embryonic lethality of approximately $70 \%$ for $\Delta 15$ animals. The expression of a KukC567S transgene decreased the lethal effects during embryogenesis to approximately $40 \%$. However, survival rate analyses of $\Delta 15$ animals which are kept over deficiency and by this only homozygous for a kuk depletion did not show an impairment of viability compared to wild type control animals. 


\subsection{Identification of biochemical interaction partners of Kuk}

The aim of this study was the identification of farnesylation dependent interaction partners of Kuk. Therefore, a Co-IP approach with a modified GFP-nanobody (provided by Prof. Görlich, MPI for biophysical chemistry, Göttingen) was used. The Großhans laboratory established a GFP tagged UAS-Kuk construct, enabling promoter specific gene expression. By this, it was known that a GFP-Kuk expression through the mat-Gal4 driver is able to rescue the kuk deficient phenotype. However, to create a situation, which is as close as possible to the native conditions, genomic GFP transgenes were generated, which are expressed under the endogenous Kuk promoter (Figure 3).

\subsubsection{GFP-Kuk transgenes did not rescue the kuk loss of function phenotype}

To determine if a GFP-tag is impairing Kuk function during cellularization, an embryo immunostaining was performed. As it was seen before in live imaging of early embryos (Figure $4 \mathrm{C}$ ) GFP-Kuk localizes to the nuclear envelope (Figure 7). A direct comparison of the nuclear envelope marker lamin Dm0 and GFP-Kuk showed the existence of a weak cytoplasmatic signal. GFP-KukC567S instead is predominantly nucleoplasmatic but also cytoplasmatic localized. However, the most important fact is that the expression of a GFP-tagged wild type allele is not able to rescue the cellularization phenotype, as it was seen with the UAS/Gal4 driven expression. These data showed that a GFP-tag is indeed affecting Kugelkern function, even if the majority of the protein is properly localized. 


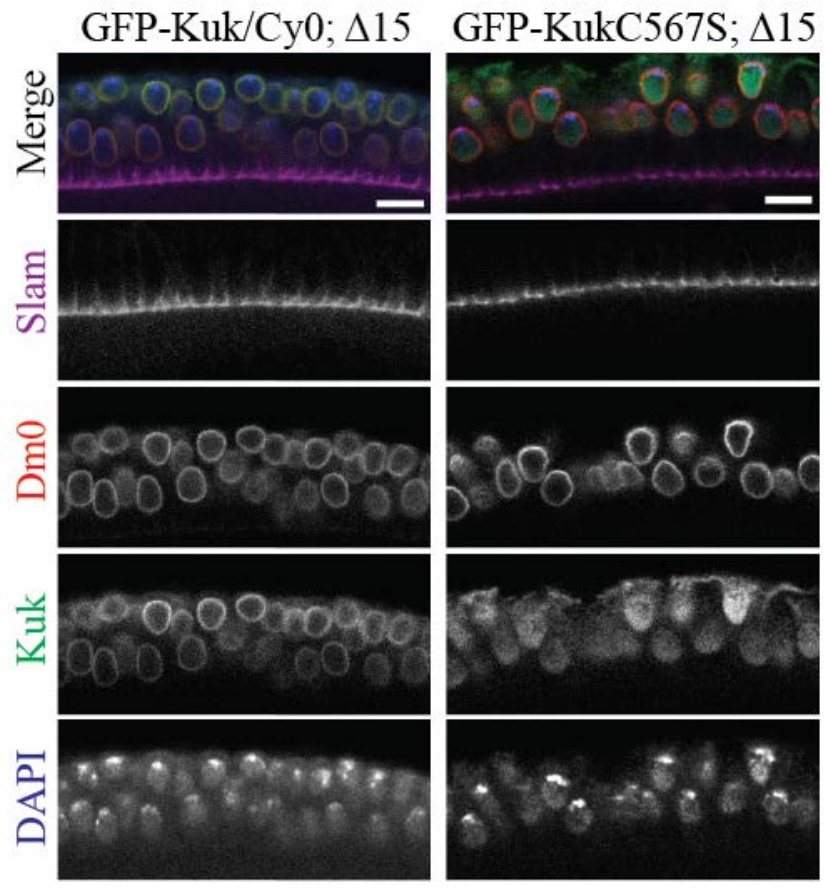

Figure 7: Genomic GFP-Kuk transgenes did not rescue the kuk-depleted phenotype

Immunofluorescence analyses for GFP-Kuk and GFP-KukC567S expressing flies in a $\Delta 15$ mutant background. Slam was used as a marker for cellularization. Dm0 and DAPI indicated the nucleus and Kuk was used to stain against the GFP-fusion proteins. In both cases a GFP-protein expression was not able to rescue the $\Delta 15$ mutant situation as the nuclei stayed roundish at the end of cellularization. A difference was observed comparing the localization of the two GFP-proteins. GFP-Kuk clearly localized to the nuclear envelope, whereas GFP-KukC567S was predominantly nucleoplasmatic but also cytoplasmatic localized. Scale bar: $10 \mu \mathrm{m}$.

\subsubsection{Proteolytic cleavage of GFP-Kuk}

To further investigate the reason for the failed complementation, western blot analysis of embryo extracts were performed and stained against Kuk and GFP (Figure 8 A). Both GFP-Kuk proteins differed in their migration behavior compared to wild type Kuk, due to their higher molecular weight. The loss of farnesylation for GFP-KukC567S was also visible in direct comparison to GFP-Kuk. However, native Kuk of wild type embryos showed only one dominant band, whereas up to three major bands were observed for the GFP-proteins. These data showed that the GFP-tag is promoting proteolytic cleavage. As the GFP-tag was fused to the N-terminal end (Figure $8 \mathrm{~B}$ ), one can conclude that degradation occurs with the C-terminal half of Kuk, as the same bands can be detected for the GFP- and the Kuk-antibody. As I was interested in binding partner which are dependent on farnesylation, mediated by the C-terminal CaaX-motif, I decided to use the maternally 
expressed GFP-Kuk protein (mat-Gal4, UAS-Kuk) for further experiments, as this was already shown to be functional and able to rescue the mutant situation.

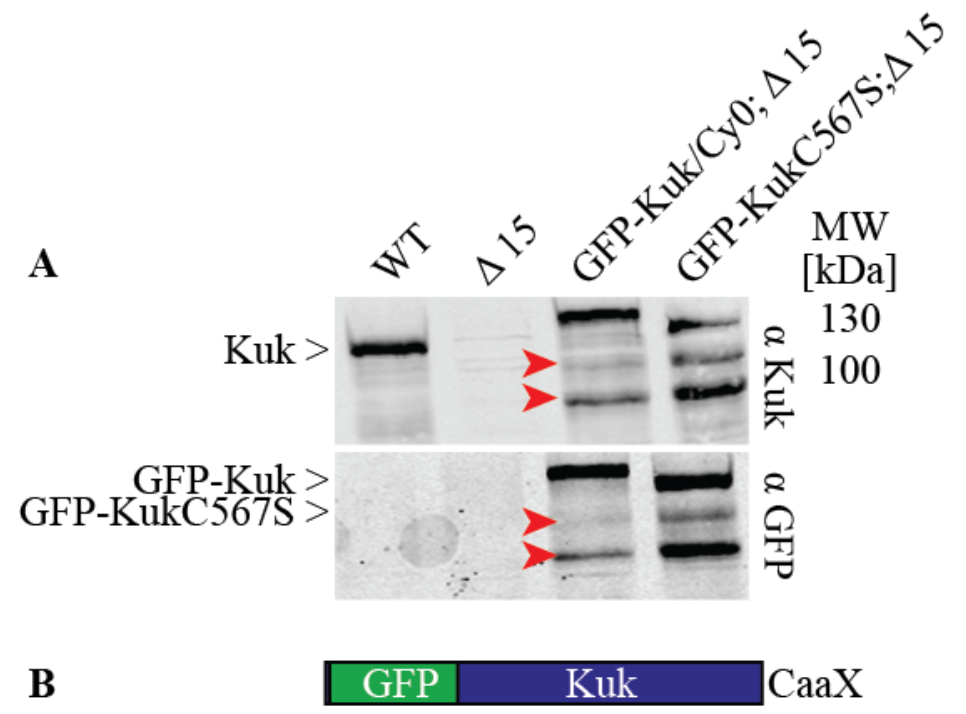

Figure 8: Protein degradation occurs at the C-terminal end of GFP-Kuk

(A) Western blot analysis of wild-type, $\Delta 15$ and GFP-Kuk(C567S); $\Delta 15$ embryo extract for Kuk (upper panel) and GFP (lower panel). The wild-type extract showed one dominant band for Kuk, but no signal for GFP. In Kugelkern deficient embryos $(\Delta 15)$ neither a Kuk nor a GFP signal was detected. Extract of GFP-transgenic embryos showed several bands, which were similar between GFP-Kuk and GFP-KukC567S (arrowhead). An extract of 40 embryos was analyzed by SDS-PAGE. (B) Schematic illustration for the generated GFP-Kuk construct. The GFP was fused to the N-terminal site of Kugelkern. The farnesylable CaaX-motif instead is localized at the C-terminus.

Furthermore, I checked if a GFP-fusion in general is affecting protein stability and also leads to degradation within UAS/Gal4 expressed GFP-Kuk. For this purpose embryo extract of the wild type Kuk was compared to the GFP-proteins expressed under the native promoter and the maternal one (Figure $9 \mathrm{~A}$ ). It is shown that degradation was present in all GFPsituations, which shared protein fragments of a similar size. Western blot analyses for the UAS/Gal4 expressed GFP-Kuk against GFP and Kuk confirmed that the cleavage products were still fused to GFP (Figure $9 \mathrm{~B}$ ) An explanation for the rescue of the UAS/Gal4 expressed GFP-Kuk was given by its expression level, which was higher and thus probably able to compensate the occurring loss of functional Kuk (Figure 9 A). At this point it has to be mentioned, that the UAS/Gal4 driven GFP-Kuk was expressed in a heterozygous $\Delta 15$ situation, which was kept over a kuk deficiency ( $\Delta 15$ / dur+,Df(3R)Ex6176), also resulting in a protein null allele for Kugelkern (following termed as D15). As the vast majority of the 
UAS/Gal4 expressed GFP-Kuk was not affected by proteolytic cleavage (Figure 9 A) and an embryo immunostaining re-confirmed its functionality (Figure $9 \mathrm{C}$ ) I used this fly strain for subsequent Co-IP analysis.

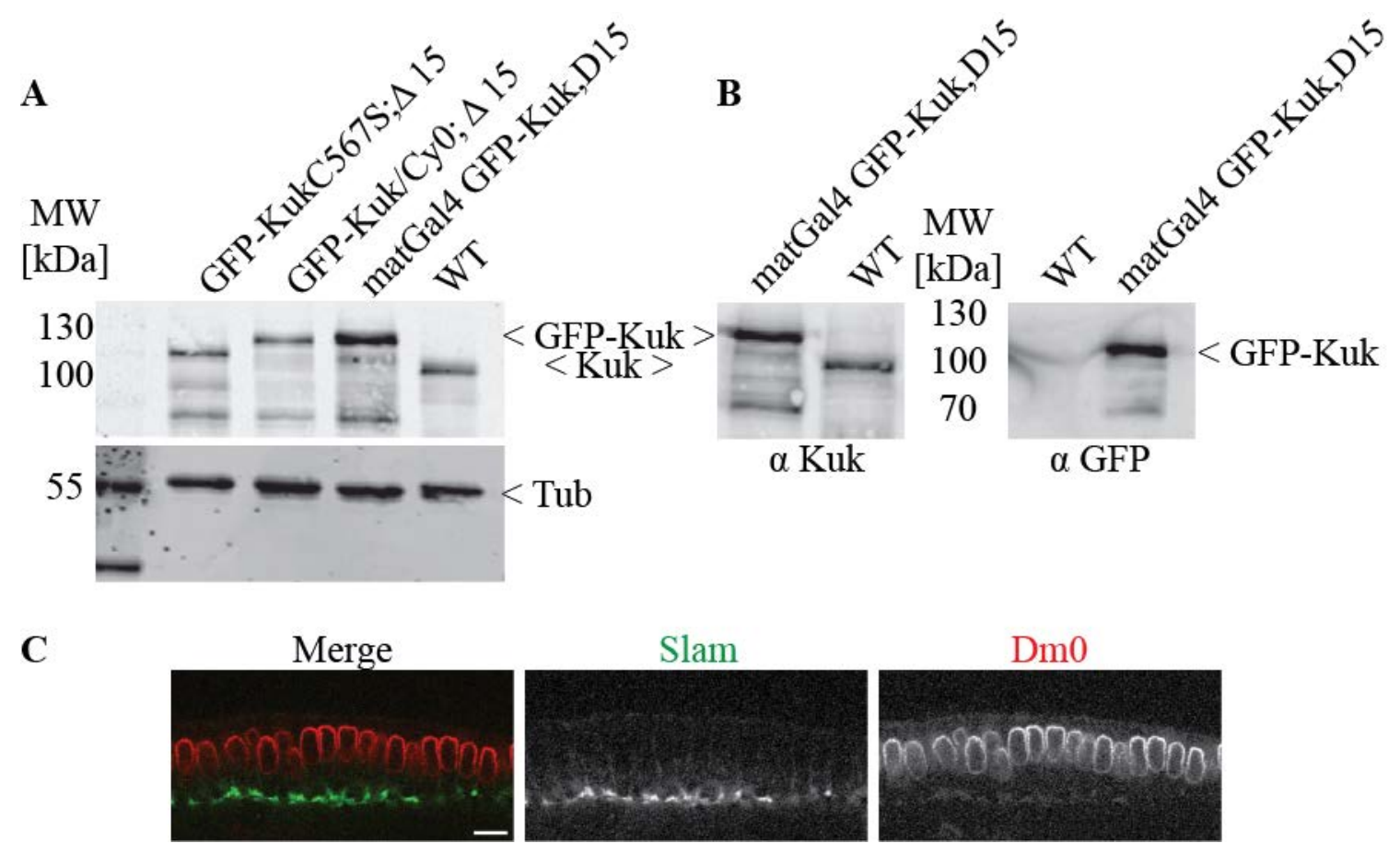

Figure 9: UAS/Gal4 expressed GFP-Kuk rescued the kuk depletion phenotype

(A) Western blot analysis of GFP-Kuk expressed under the native and the maternal promoter compared to extract of wild type embryos. Western blot analyses were performed against Kugelkern and $\alpha$-tubulin, which was used as loading control. GFP-proteins under the native promoter were expressed in a homozygous $\Delta 15$ background ( $\Delta 15$ ), whereas the maternal GFP-Kuk protein was expressed in a heterozygous $\Delta 15$ situation, kept over deficiency (D15). Comparisons of the GFP-Kuk bands showed a similar appearance, especially regarding the cleavage products. Wild type Kuk and natively expressed GFP-Kuk proteins had a similar band intensity. Maternal GFP-Kuk showed a higher intensity correlating with a stronger expression. (B) Western blot analysis of the maternally expressed GFP-Kuk stained against GFP and Kuk revealed that the Kuk cleavage products were still fused to GFP. An extract of 40 embryos was loaded for the analyses shown in A and B. (C) Side view of Drosophila embryos stained against Slam, a cellularization marker, and lamin Dm0 a marker for the nuclear envelope. Maternally expressed GFP-Kuk rescued the nuclear elongation phenotype in a kuk depleted background (D15). 


\subsubsection{Nuclei solubilization promoted further degradation}

The identification of interaction partner by biochemical approaches is critically dependent on the experimental conditions. One of the crucial factors is the salt concentration, which will influence protein-protein interactions. It was shown before that Kuk can be extracted from the nuclear envelope by using $150 \mathrm{mM}$ of $\mathrm{NaCl}$ (Brandt et al., 2006). Another important point which should be considered are unspecific background signals. In this approach a modified GFP-nanobody (provided by Prof. Görlich, MPI for biophysical chemistry, Göttingen) was used. The big advantage of this camelid derived single domain antibody is an intrinsically SUMO cleavage site, which enables an additional purification step and reduces unspecific binding. This is especially important for subsequent mass spectrometric analysis. To finally identify interaction partner, whose binding is determined by the farnesylation it was planned to compare isolated proteins of GFP-Kuk and GFPKukC567S. Unspecific binding will be excluded by using the extract of kuk deficient embryos as control.

A nuclei fractionation experiment with subsequent co-immunoprecipitation is shown in Figure 10. Analyses of the total lysate of GFP-Kuk embryos again showed the full-length protein as well as its cleavage products, termed as GFP-Kuk $\Delta$ C-term. Enrichment of full length GFP Kuk was possible by fractionating embryonic nuclei (Figure 10, Nuclei). The input represents a protein sample after treating the isolated nuclei with $150 \mathrm{mM} \mathrm{NaCl}$ and 1\% Triton $\mathrm{X}$ to extract the GFP-Kuk protein from the nuclear envelope. This solubilization step was essential for subsequent co-immunoprecipitation. However, nuclei solubilization severely affected GFP-Kuk protein stability and promoted degradation (Figure 10, red arrow). Consistent to this, a Kuk fragment with a molecular weight of around $55 \mathrm{kDa}$ was enriched in the unbound sample after adding the GFP-input sample to the GFP-nanobody coupled beads. Elution was achieved by using the intrinsic SUMO cleavage site of the GFPnanobody. Eluted GFP-Kuk protein was detected in the cleaved fraction (Figure 10, arrow). It was shown that GFP-Kuk can be efficiently isolated with this approach. However, as a GFP-Kuk fragment (Figure 10, red arrow head) was enriched due to occurring protein cleavage this approach had to be stopped. Thus, protein cleavage became a severe problem which prevented the identification of farnesylation dependent interaction partner of Kugelkern. 


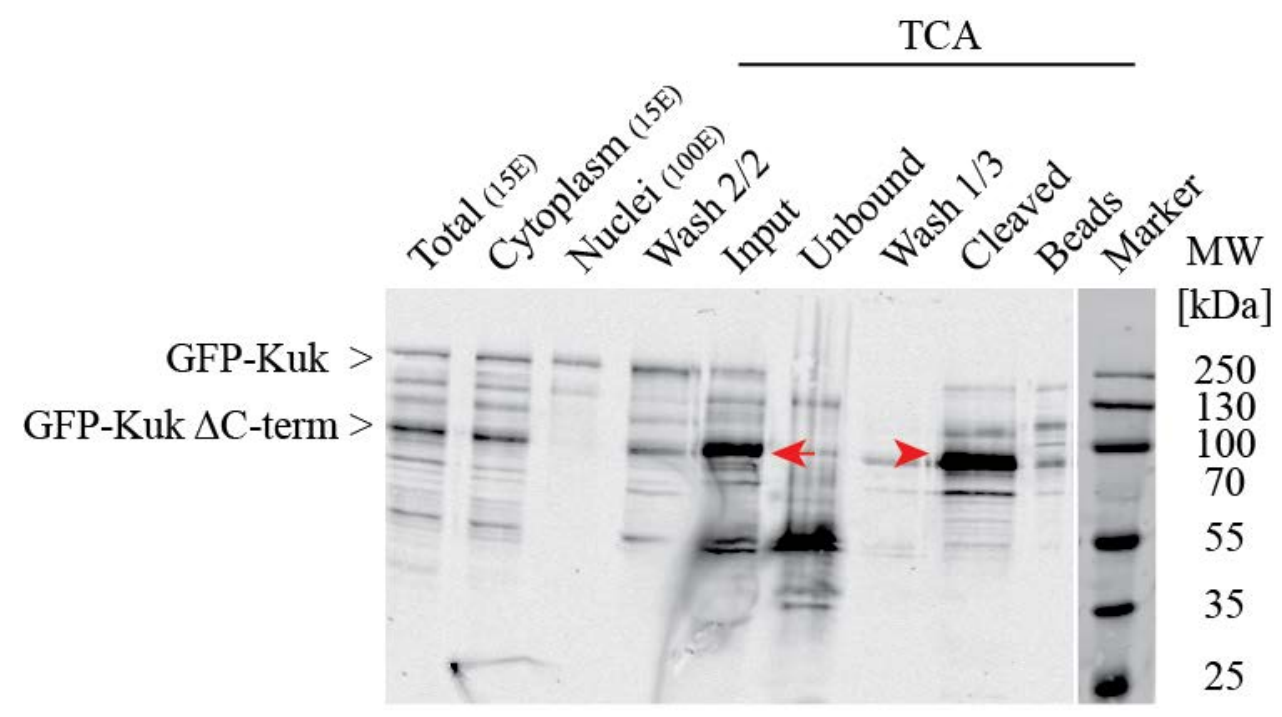

Figure 10: Solubilization promoted GFP-Kuk degradation

Western blot analysis of a nuclei fractionation experiment with subsequent co-immunoprecipitation using lysate of GFP-Kuk expressing embryos. GFP-Kuk was identified by Kuk antibody staining and detected in the nuclei fraction as well as in the cytoplasm, due to insufficient separation. Full length GFP-Kuk was enriched by nuclei fractionation. Solubilization was achieved using $150 \mathrm{mM} \mathrm{NaCl}$ and 1\% TritonX 100 (input, 4.500 E). After solubilization the majority of the GFP-protein was proteolytic cleaved (red arrow). The input sample was further incubated to the GFP-nanobody coupled beads. The protein fraction which was not able to bind is shown in the unbound fraction. Three washing steps were performed and the first washing step is shown (wash 1/3). GFP-Kuk elution was achieved by SUMO cleavage (Cleaved, red arrow head). Protein which was not eluted is shown in the beads fraction. Trichloroacetic acid (TCA) was used for protein precipitation of the indicated protein fractions.

An alternative to the above mentioned approach is a co-immunoprecipation of the native and mutant Kugelkern by a Kuk antibody coupled to Protein A dynabeads. In order to identify farnesylation dependent interaction partners, transgenic KukC567S flies without GFP were generated and crossed in the kuk deficient situation $(\Delta 15)$. Solubilization analyses of the native Kuk revealed that this approach is promising as the majority of the Kuk protein stayed stable for even 4 hours after solubilization (Supplement Figure 2). First Co-IP experiments revealed that this system is working and I am confident to study Kugelkern protein isolates by mass spectrometric analysis in the near future. 


\subsubsection{Biochemical properties of GFP-Kuk (C567S)}

Next, FRAP experiments were performed, using the established GFP transgenic lines for Kuk and KukC567S, to analyze the protein dynamics of Kugelkern. As the C567S mutation is changing the Kugelkern localization from the nuclear lamina to the nucleoplasm it was assumed that it also changes biochemical properties. In order to identify protein dynamics a circular area was bleached and the recovery rate was measured every 10 seconds over a time period of 10 minutes. To include the bleaching effect which was induced by image recording the fluorescence intensities of two control areas were measured and used for normalization.

The GFP signal of both GFP-proteins was efficiently bleached (Figure $11 \mathrm{~A}, \mathrm{~B}$ after bleach). After 10 minutes a signal recovery rate of $\sim 25 \%$ for the wild type version and $\sim 50 \%$ for the mutant version were observed. Thus, preventing a proper Kuk localization led to a more dynamic behavior. An interesting finding for the wild type allele is, that it recovers relative fast especially in direct comparison with lamins. For the GFP tagged C.elegans lamin (Celamin) no recovery after more than 60 min have been observed (Wiesel et al., 2007). Similar results are published for the human lamins $\mathrm{A}\left(\mathrm{t}_{1 / 2} \sim 140 \mathrm{~min}\right), \mathrm{B} 1\left(\mathrm{t}_{1 / 2}>180 \mathrm{~min}\right)$ and the Drosophila lamin Dm0, indicating a large immobile fraction (Gilchrist et al., 2004, Moir et al., 2000, Zaremba-Czogalla et al., 2012). 
A GFP-Kuk/Cy0; $\Delta 15$
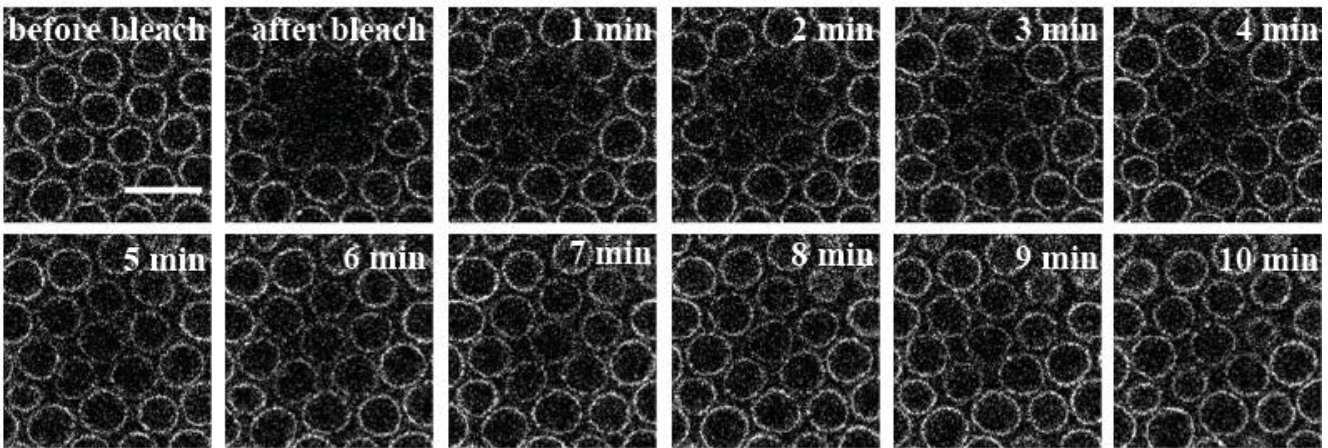

B GFP-KukC $567 \mathrm{~S} ; \Delta 15$
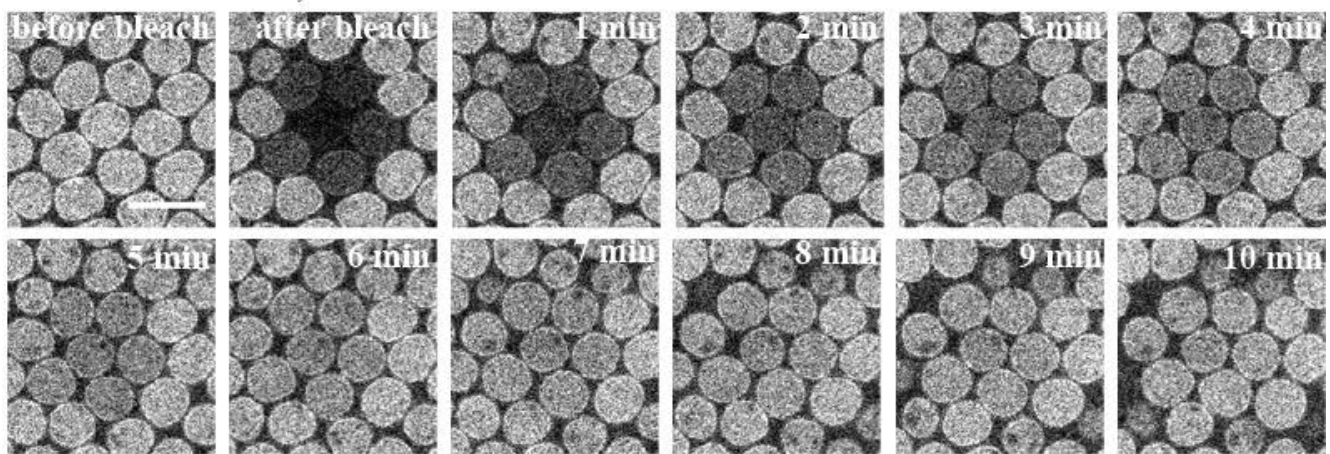

C
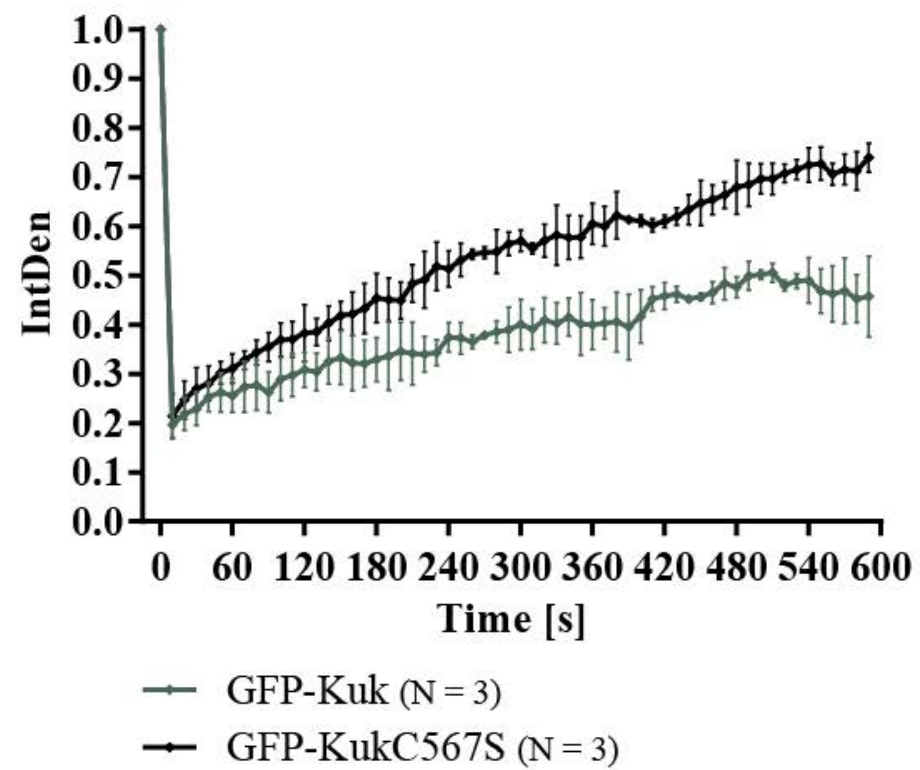

Figure 11: FRAP analysis of wild type and mutant GFP Kuk

FRAP experiment of early embryos expressing either genomic GFP-Kuk (A) or a non-farnesylable GFP-KukC567S mutant (B). Signal intensity was measured before and after photobleaching and subsequently every $10 \mathrm{~s}$ over a time period of $10 \mathrm{~min}$. Fluorescence recovery rate was measured and analyzed using ImageJ. Schematic illustration was done with GraphPad Prism6 (C). Scale bar: $10 \mu \mathrm{m}$. 


\subsection{Identification of genetic interaction partners of Kugelkern}

An aim of this work was the identification of novel genes interacting with kugelkern. Tissue-specific overexpression of Kugelkern is known to induce morphological defects (Figure 12) (Brandt et. al., 2008). To identify kugelkern interacting genes a genetic screen for dominant suppressors in the wing as well as in the eye was performed. Tissue specific overexpression of Kuk was achieved using a UASt-Kuk driven by vestigial-Gal4, which was expressed in the dorso-ventral compartment border of larval wing discs. The GMR-Gal4 (glass multiple reporter) was used for expression within the larval eye imaginal discs. The hypothesis was that other downstream targets mediate the lobulations and infoldings which have been induced by Kuk overexpression (Brandt et. al., 2008). For this dominant screen, around 130 RNAi-lines (Supplement Figure 2) for genes concerning nuclear functions were employed. Two tissues were used due to the different gene functions of the tested RNAicandidates, which could for example prevent the suppression of the induced phenotype in the wing, but could be observed in the eye. Drosophila is a relatively simple model organism and genetic analyses can be studied easily. The big advantages of this approach were first a fast screening of a high number of RNAi-lines and second the immediate identification of a candidate after suppressing the Kugelkern induced gain-of-function phenotype. The work flow of the performed RNAi-screen is depicted in Figure 12. 

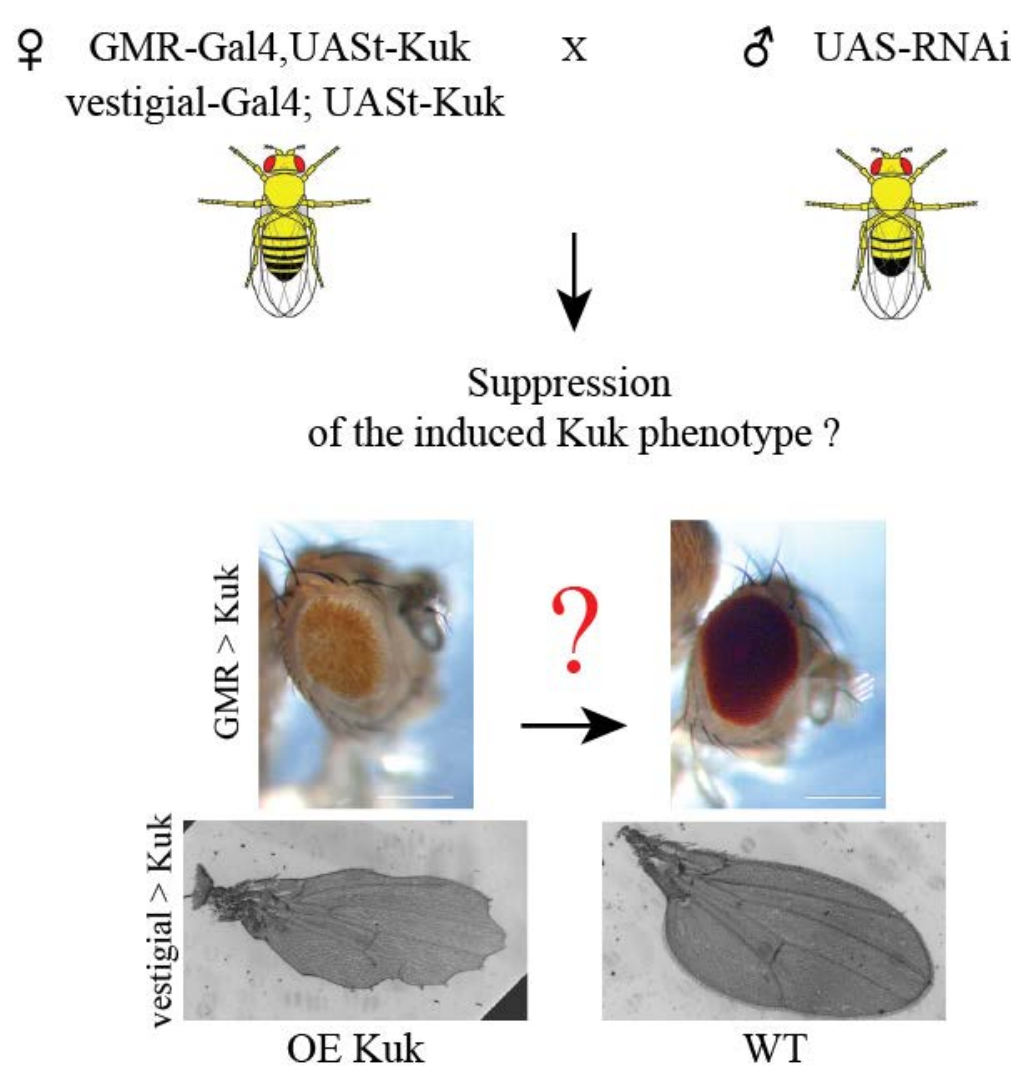

Figure 12: Scheme of the performed RNAi-screen

Virgins overexpressing Kugelkern controlled by vestigial-Gal4 or GMR-Gal4 were crossed to males containing a candidate specific RNAi construct. F1-progeny were checked for their genotype to contain the Gal4-driver, the UASt-Kuk and the UAS-RNAi construct and screened for their tissue-specific phenotype. A genetic interactor of Kuk can be identified if the induced morphological defect is reverted. Fly clip art was taken from Kondo and Ueda, 2013.

\subsubsection{Morphological defects upon Kugelkern overexpression were primary independent of apoptosis}

A simple explanation for the observed gain-of function phenotypes could be that Kuk overexpression directly resulted in apoptotic processes, leading to morphological deformations. To rule this out, immunofluorescence analyses of wing- and eye-imaginal discs were performed. Apoptotic events were detected by Caspase-3 staining. Overexpression of Reaper, which has a central role in programmed cell death, was used as positive control. Wing-specific overexpression of Reaper led to an increase in Caspase-3 signal and finally caused adult wing deformations (Figure 13). Expression of DIAP, the Drosophila inhibitor of apoptosis, in combination with Reaper, resulted in a reduced Caspase-3 staining and wing formation occurred to be normal. Overexpression of Kuk also increased Caspase-3 staining in wing discs of stage 3 larvae. However, additional expression 
of DIAP reduced but did not prevented Caspase-3 activation and still resulted in wing defects, suggesting that next to apoptosis also other processes were involved and mediated the observed phenotypes.

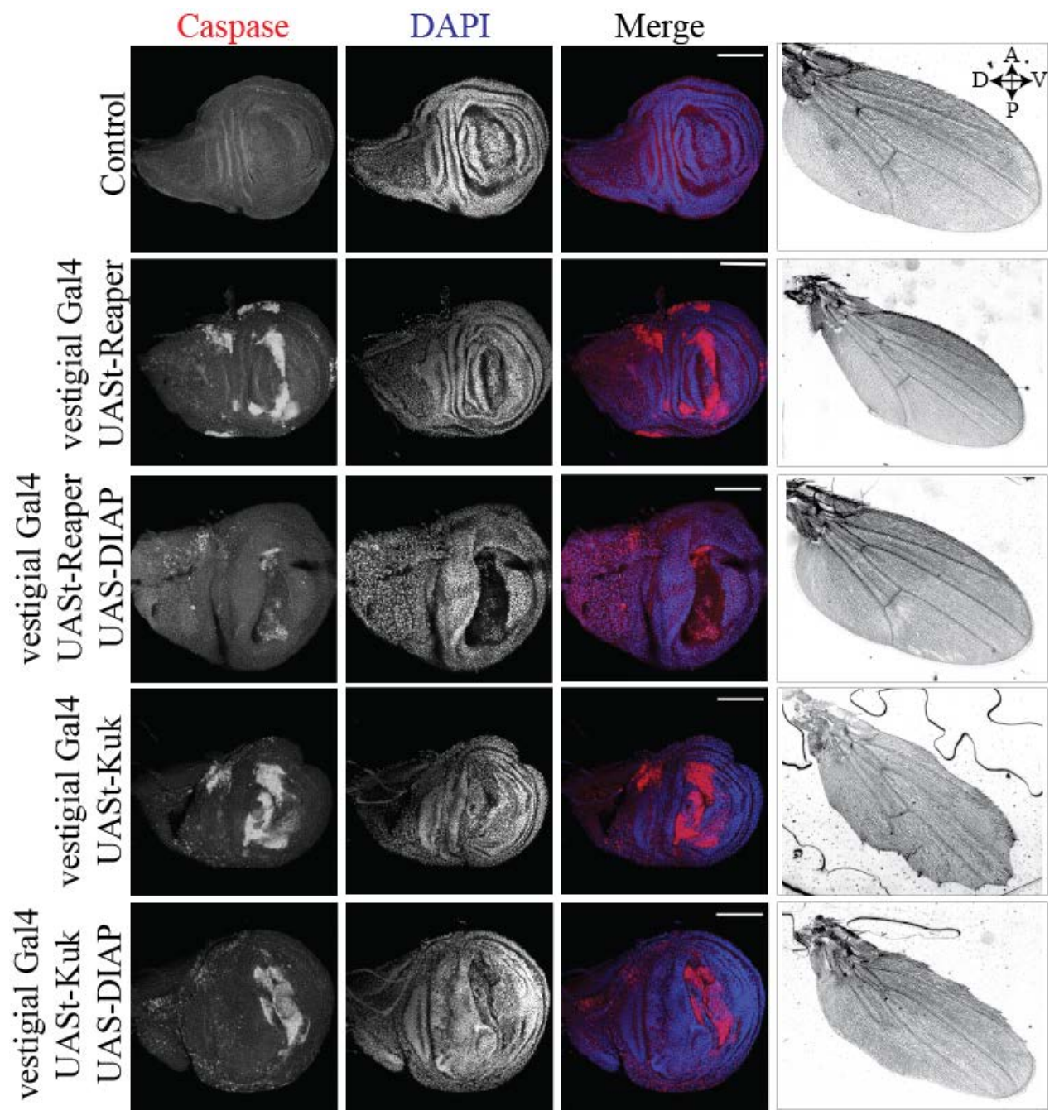

Figure 13: Kugelkern overexpression caused wing morphological defects

Immunofluorescence analyses of wing imaginal discs. L3 larvae of the depicted genotypes were stained against Caspase-3, as marker for apoptotic events, and DAPI was used as a DNA marker. Wild type discs were used as control and did not show a Caspase-3 activation. Wing specific expression of Reaper led to apoptosis and was used as positive control. Expression of an apoptotic inhibitor, DIAP, in combination with Reaper decreased Caspase-3 activation and resulted in a normal wing development. Kuk overexpression increased Caspase-3 intensity, which led to wing deformations, also in combination with DIAP.

The effect of Kuk overexpression referring to induction of apoptosis was also checked for eye-imaginal discs by Caspase-3 immunostaining. It was observed that the induction of the 
eye phenotype was dose-dependent as a homozygous expression of GMR-Gal4, UASt-Kuk induced a stronger phenotype than the heterozygous situation (Figure 14). But, flies which were homozygous for GMR-Gal4, UASt-Kuk could be kept but not amplified for the RNAi-approach as they were strongly affected in viability. However, if the eye deformation is a result of directly induced apoptotic events, the effect for the Caspase-3 activation is expected to be stronger in the homozygous GMR-Gal4, UASt-Kuk situation than the heterozygous one. Due to this eye-imaginal discs of homozygous animals were analyzed. Ubiquitous GFP-cadherin expressing animals were used as control (Figure 14 B) and stained in the same tube together with Kuk overexpressing ones (Figure $14 \mathrm{C}$ ). As there was no induction of apoptotic events during larval development (Supplement Figure 3), eye imaginal discs of prepupae were tested. Immunofluorescence analysis showed a weak signal for Caspase-3 in control discs, which was slightly increased in the Kuk overexpressed situation. However, these events occurred only in a dot like manner and were not as strong as in the wing imaginal discs before (Figure 13). These results indicated that Kuk overexpression indeed partially resulted in apoptosis but it seemed that this was a consequence and not the primary reason of the observed morphological defects. 

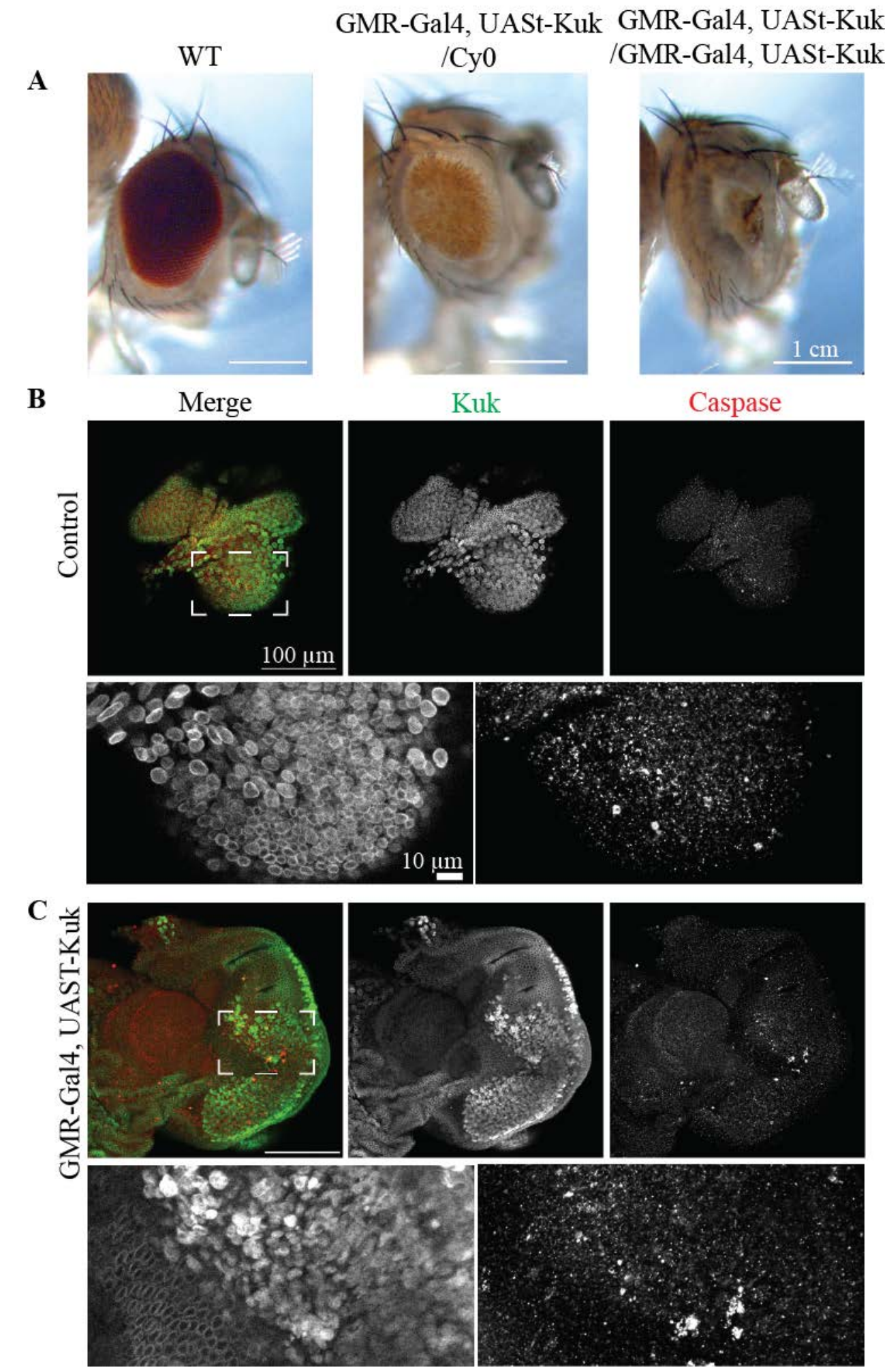

Figure 14: Kuk overexpression induced eye-defects primary independent of apoptosis

Eye morphological defects were induced by Kuk overexpression in a dose-dependent manner. (A) Eye phenotypes of wild type flies and flies overexpressing Kuk in a homozygous and heterozygous situation controlled by the GMR promoter. (B-C) Eye imaginal discs of a ubiquitous GFP expressing control situation (B) and an eye specific Kuk overexpressing situation (C). Immunofluorescence analyses were performed 
against Kuk and Caspase-3. Caspase-3 activation was detected on a very low level for the control as well as the Kuk overexpressing situation.

\subsubsection{Genetic interaction partners of Kuk remain undiscovered}

To finally search for genetic interaction partners of $k u k$ the RNAi-screen was performed (Figure 12). Therefore, flies overexpressing Kuk were crossed with around 130 candidates for genes concerning nuclear functions, which were available in the RNAi-library of the Großhans laboratory (Supplement Figure 2). The females of the parental generation were heterozygous (vestigial-Gal4/CyO, UASt-Kuk/UASt-Kuk or GMR-Gal4, UASt$\mathrm{Kuk} / \mathrm{CyO}$ ), meaning only half of the progeny were screened as the other half contained the balancer chromosome and did not overexpress Kuk. The big advantage of this situation was that an internal control for each candidate was directly provided. However, within all tested candidates, no genetic interaction partner was found for Kuk, neither in the wing nor in the eye. For all screened wings at least the proximal posterior region was affected (Figure $15 \mathrm{a}-\mathrm{j}$ ). Only the knockdown of Kuk itself, which was used as control, rescued the gain-of-function phenotype (Figure 15 e-f). The same effect was seen for the performed screen in the eye (Figure $15 \mathrm{k}$-p). These results showed first, that the number of tested candidates was too small and second, that the principle of the RNAi-screen in general worked. 
A

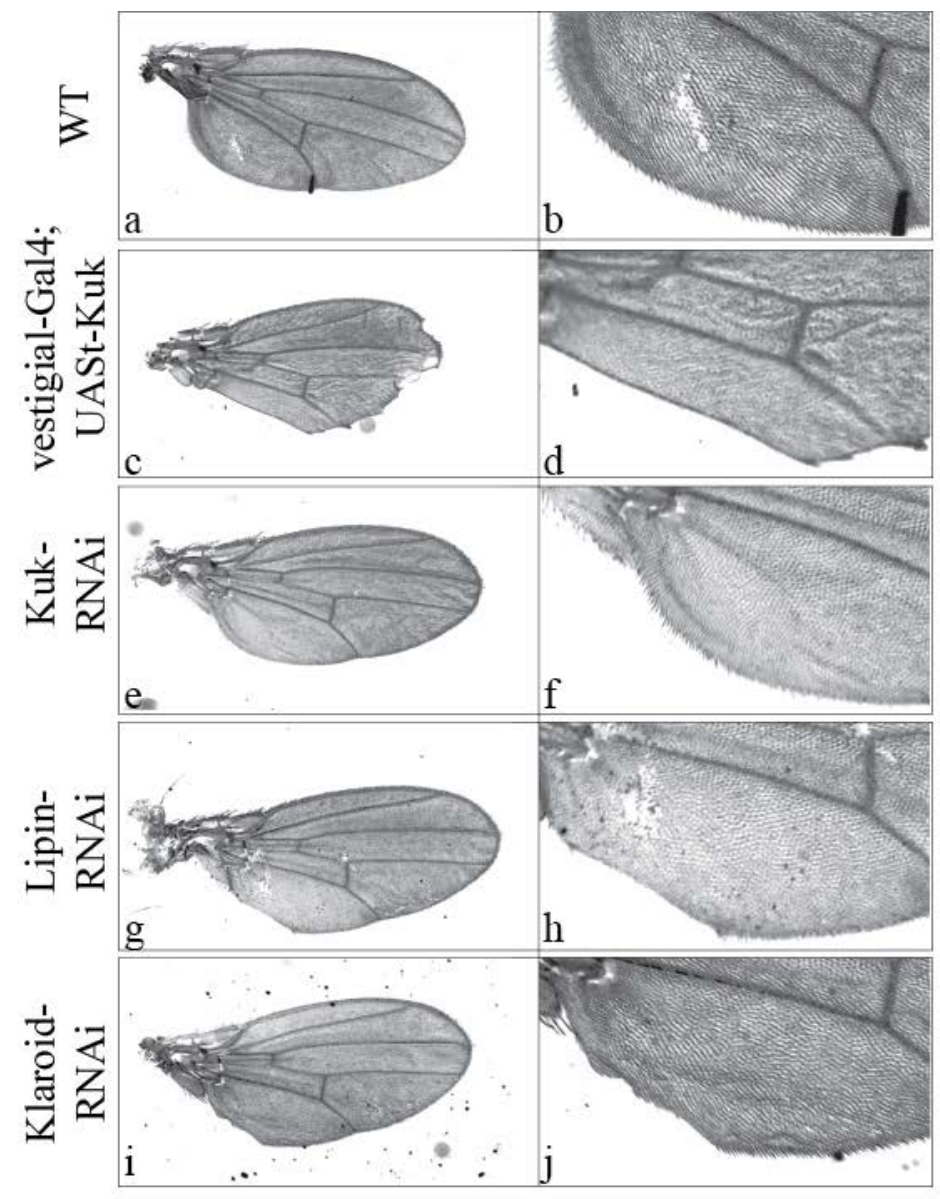

B Control

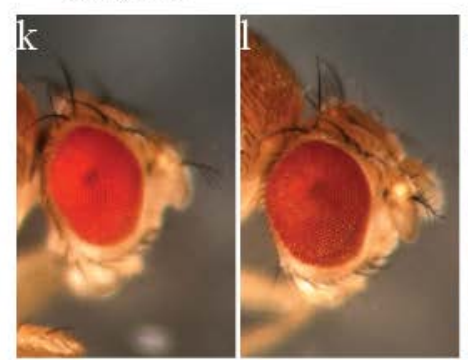

Kuk-RNAi
Control

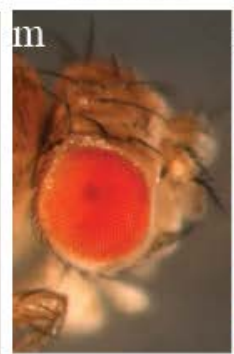

Mad-RNAi
Control
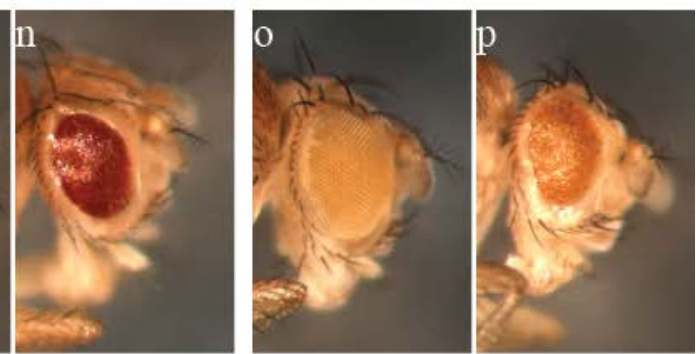

Nucleoplasmin-RNAi

Figure 15: Genetic interaction partners of kuk remain unidentified

(A) Genetic screen performed for the wing. (a) Wings of wild type flies were used as control. (c) Wing specific overexpression of Kugelkern induced morphological defects, which were reverted by a knockdown of Kuk (e). (g-j) Wing pictures of Lipin- and Klaroid RNAi expressing flies in the background of Kuk overexpression. (b, $\mathrm{d}, \mathrm{f}, \mathrm{h}, \mathrm{j}$ ) Representative pictures of the magnified wing area which was affected in all tested candidates. (B) Genetic screen performed for the eye. Representative pictures of the eye-phenotypes of flies expressing an RNAi construct for Kuk, Mad or Nucleoplasmin in the background of Kuk overexpression (l, n, o). Kuk-RNAi (l) was used as positive control and restored the gain of function phenotype. Knockdown of other candidates concerning nuclear function had no effect e.g. Mad (n) or GD22623 (p). The internal control of each situation was used as direct comparison ( $\mathrm{k}, \mathrm{m}, \mathrm{o})$. 


\subsection{The role of Narf in nuclear morphogenesis and aging}

Narf was identified as a farnesylation dependent binding partner of human PrelaminA (Barton and Worman 1999). Recently Prof. Wollnik and coworkers revealed that a dominant de novo mutation of human Narf causes clinical symptoms that share similarities to HGPS a human premature aging syndrome. In this study I tested the hypothesis that Narf is an interactor of farnesylated Kugelkern and Lamin and established a Narf disease model.

\subsubsection{Generation of a Narf specific antibody}

The Drosophila homologue of Narf is CG17683, hereinafter referred as Narf. Experiments based on antibodies are indispensable for understanding protein functions. Due to this I generated a Narf specific antibody. The cDNA clone RE37350 was used as the template for PCR and the coding sequence of Narf was inserted into QE80N60 vector. The recombinant protein was expressed in bacteria for four hours at $37^{\circ} \mathrm{C}$ induced by $1 \mathrm{mM}$ IPTG. E. coli cells were lysated by a combination of freeze and thaw, enzymatical lysis by lysozym and mechanical homogenization using the microfluidizer (Figure $16 \mathrm{~A}$ ). As the majority of the protein stayed in the insoluble pellet fraction $(\mathrm{P})$ a denatured protein purification using His-tag and $8 \mathrm{M}$ urea was performed (Figure $16 \mathrm{~B}$ ). Immunization was subsequently done by BioScience into guinea pig and rabbit and the final sera were tested in western blot analyses for their specificity (Figure $17 \mathrm{~A}$ ). Three of the four final sera showed a number of unspecific bands, which were even visible in a high antibody dilution (sera of gp 02 and rabbit 1634, 1635). One serum, the gp 01, showed a predominant band at the expected size of $\sim 55 \mathrm{kDa}$. To further verify the Narf specificity for this antibody a clonal analysis using the flip out system was performed. The basic principle behind that assay is a temperature dependent expression of a clonal marker and a flippase. The flippase enables specific expression of a desired UAS gene sequence by inducing a recombinatory flip out (Jiang et al., 2009). This systems was used for a clonal overexpression of Narf in the Drosophila gut. GFP was used as clonal marker and indicates Narf overexpressing cells. The big advantage of this approach is a direct comparison of Narf overexpressing and control cells, which locate next to each other in the same tissue and could be stained and imaged under same conditions. It is shown in Figure 17 B that the generated Narf antibody indeed recognized an elevated protein level in a Narf overexpressed situation, which is overlapping with the GFP control signal. This result strongly indicates a Narf specificity of the new antibody. 
$\mathbf{A}$

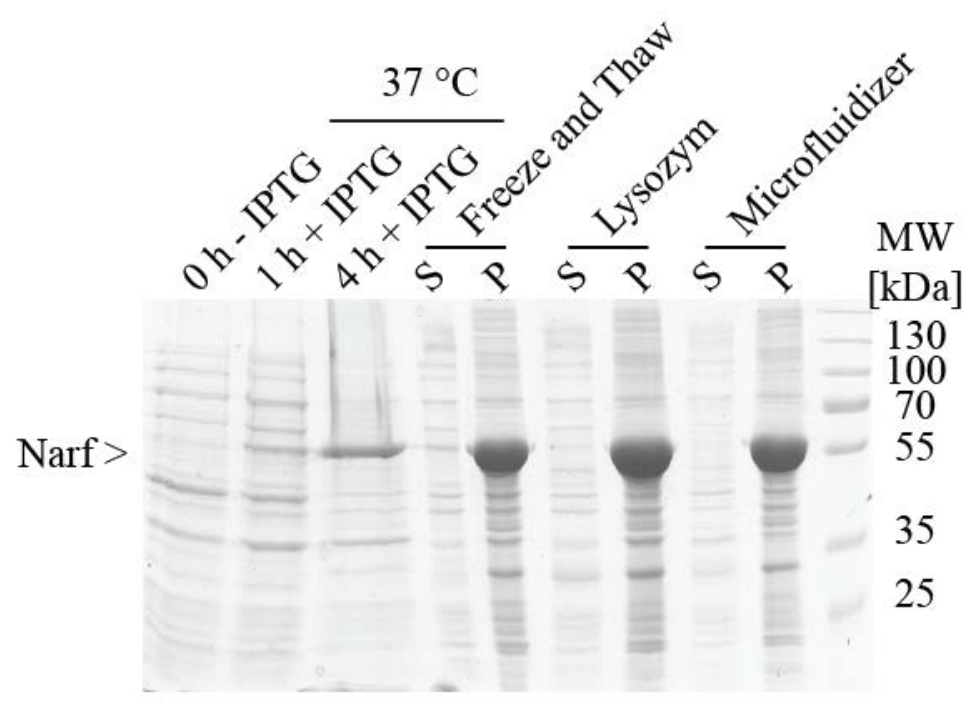

B

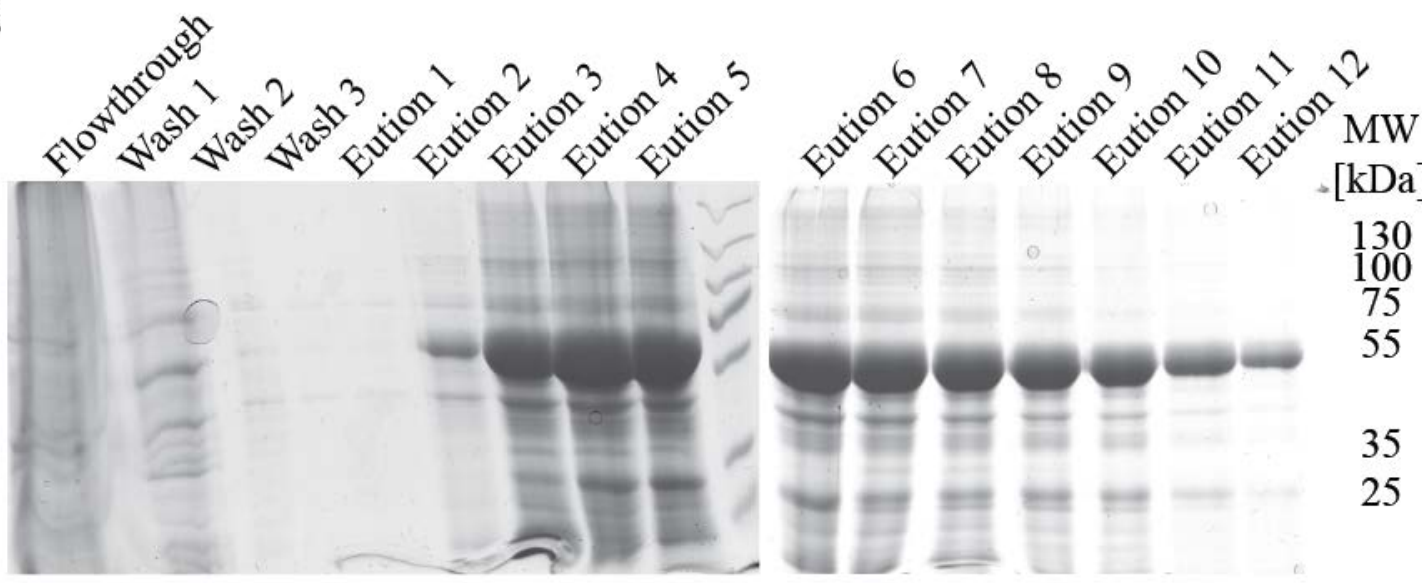

Figure 16: Protein expression and purification of QE80N60-Narf

(A, B) SDS-PAGE analysis of Narf expression and purification. (A) Narf expression was performed for 4 hours at $37^{\circ} \mathrm{C}$ induced by $1 \mathrm{mM}$ IPTG. Cell lysis was done by freeze and thaw, enzymatical lysis using lysozyme and mechanical homogenization using the microfluidizer. Comparison of the soluble supernatant fraction (S) and the insoluble pellet fraction $(\mathrm{P})$ showed a high insoluble protein amount. According to this protein purification was performed under denatured conditions using His-Tag (B). Elution was achieved by a change in $\mathrm{pH}$-value. Narf protein containing fractions were collected and used for immunization. 
A

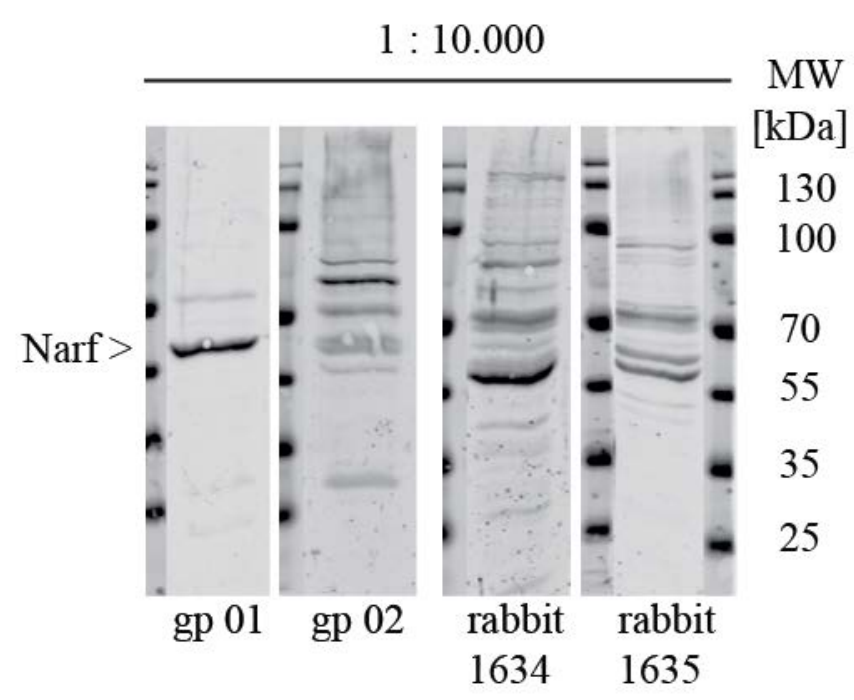

B EsgTS/UASt-Narf WT; F/O

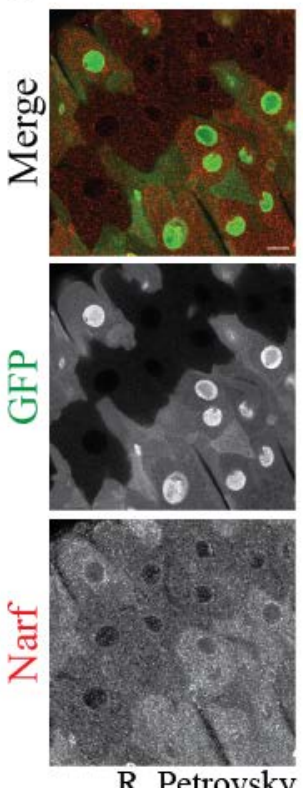

Figure 17: The antibody stained specific for Narf

(A) Western blot analysis of embryo extract stained for Narf using the different sera with the indicated dilution and batches name. Besides gp 01 all sera show a high amount of unspecific binding. (B) Immunofluorescence analysis of a clonal, Narf overexpressing gut stained for Narf using the sera gp 01. Scale bar: $10 \mu \mathrm{m}$. The clonal analysis was conducted by R. Petrovsky.

\subsubsection{Narf localized to the nuclear envelope}

In order to identify Narf localization immunofluorescence analyses using the generated antibody were performed. S2 cells as well as early Drosophila embryos were studied. Co-stainings for nuclear envelope markers such as lamin Dm0 or Nup153 showed that Narf clearly localized to the nuclear envelope (Figure 18 A-D). Another evidence that the antibody stains specifically for Narf was given by the observation that the signals, of the Narf antibody and a mCherry tagged Narf protein expressed in S2 cells, show similar results (Figure 18 A). Airy Scan microscopy of fractionated embryonic nuclei and embryonic amnioserosa cells showed that Narf localization resembles the lamin Dm0 localization pattern and both proteins are partially overlapping in their local distribution (Figure $18 \mathrm{C}-\mathrm{D}$ ). Since Narf and Dm0/Kuk are locating within the same nuclear area, it is possible that they are also interacting with each other. However, unlike initially described for human Narf (Barton and Worman, 1999), the Drosophila homologue is not restricted to the nuclear envelope but can be also found within the cytoplasm. This observation was given by immunofluorescence analyses and confirmed by fractionation experiments of embryo 
extract (Figure 18 E). Western blot analysis showed that the majority of Narf is cytoplasmatic but a distinct proportion can be also found in the nuclear fraction, together with lamin Dm0, which was used as nuclear fraction marker.

A

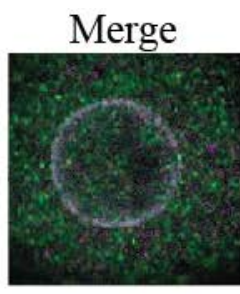

Narf

Nup153 mCherry-Narf
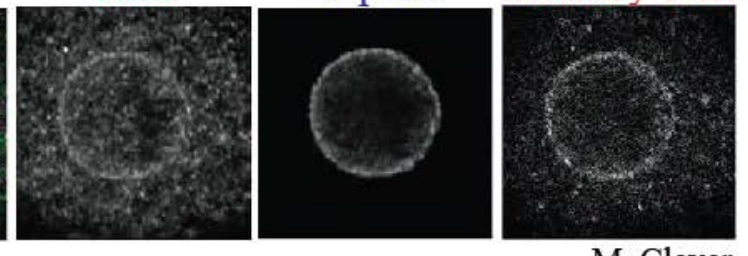

M. Clever

B

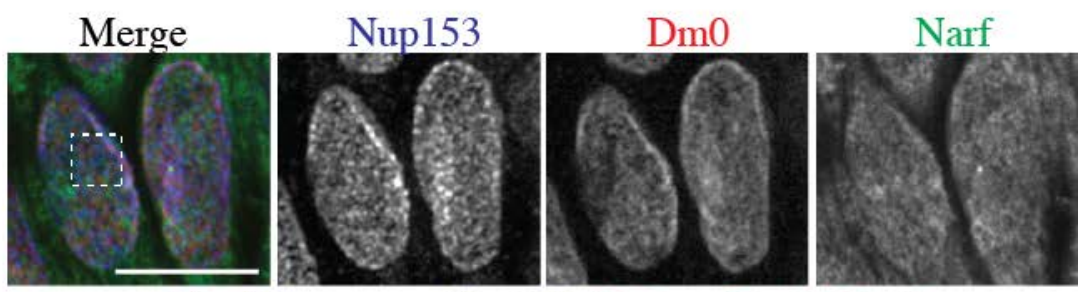

C

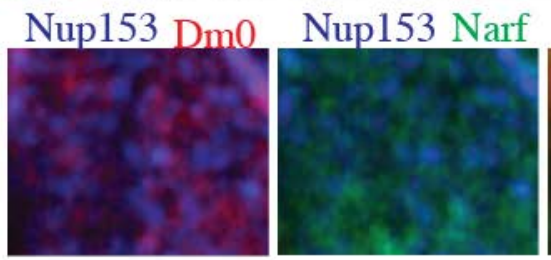

Dm0 Narf

D

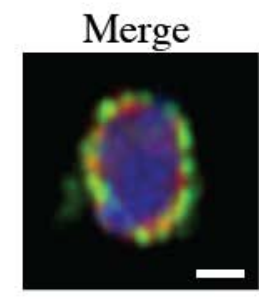

Narf

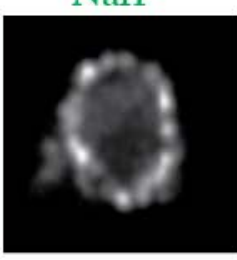

Dm0
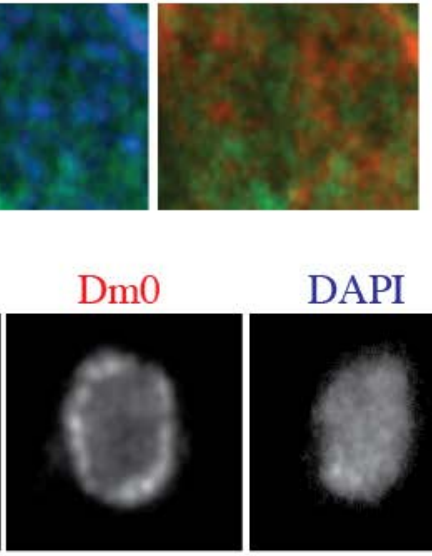

DAPI

E
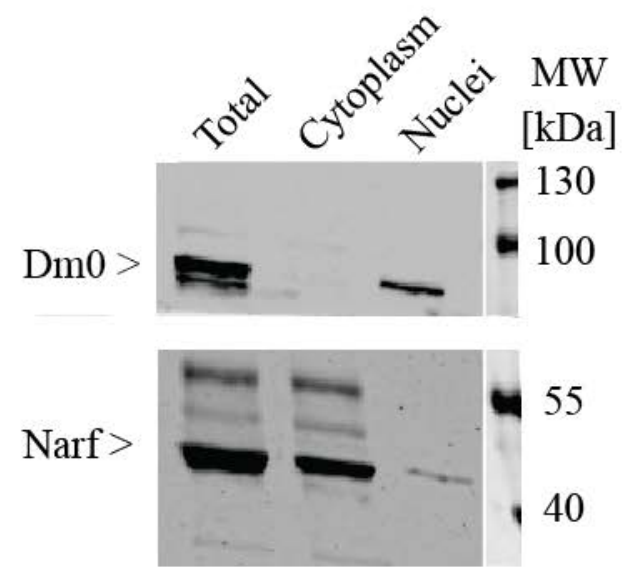
Figure 18: Narf localized to the nuclear envelope

Immunofluorescence analyses of S2 cells (A), embryonic amnioserosa cells (B, Scale bar: $10 \mu \mathrm{m}$, magnification depicted in C) and fractionated embryonic nuclei (D, Scale bar: $1 \mu \mathrm{m}$ ) show that Narf localizes to the nuclear envelope. Airy Scan microscopy of fractionated nuclei (D) revealed that Narf distribution partially overlaped with Dm0 and is different from Nup153 localization. Immunofluorescence analyses were confirmed by a fractionation experiment performed with wild type embryo extract (E). Western blot analysis showed a predominant signal within the cytoplasmic fraction. Nevertheless, a distinct signal was also observed within the nuclear fraction. Success of fractionation was given by Dm0 detection. The lysate of 25 embryos was loaded for each fraction. Generation of a mCherry-tagged Narf version and subsequent immunofluorescence analysis of S2 cells were performed by M. Clever.

\subsubsection{Narf has a function during Drosophila development}

Narf is known to possess an essential function for viability in Drosophila, since four different lethal alleles (NC37, NC38, NC70 and NC109) have been already identified (Coulthard et al., 2010). However, only NC38 and NC109 are further described and known to contain mutations within the third exon. In the allele NC109 two nucleotide substitutions cause amino acid exchanges from valine (V) to aspartic acid (D) at position 152 and serine (S) to alanine (A) at position 154. NC38 possesses a nonsense mutation as it introduces a stop codon (*) at position 166 (Figure 19 A). To study Narf function during development I used flies containing these lethal alleles, which are available at the Bloomington Stock Center. In order to document the potential mutant Narf region in NC37 and NC70 genomic DNA containing Narf was sequenced. Even if it was possible to reproduce the previous described data for NC38 and NC109, I did not find any mutation within the coding region of the uncharacterized alleles NC37 and NC70 (Figure 19 B). 
$\mathbf{A}$

NC109

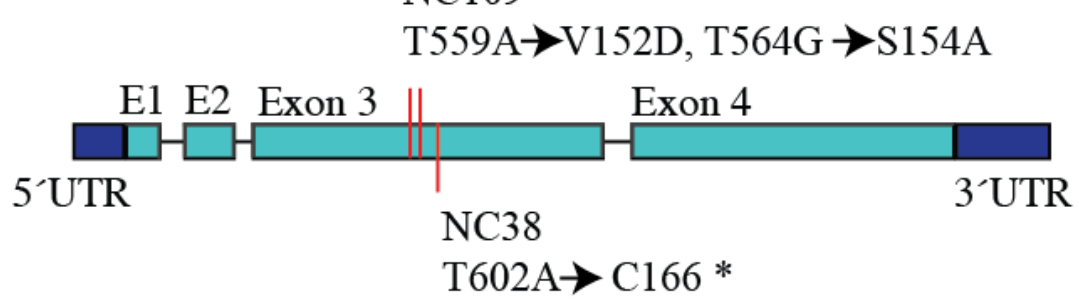

B $\quad \mathrm{NC} 38$

C $\quad$ NC109
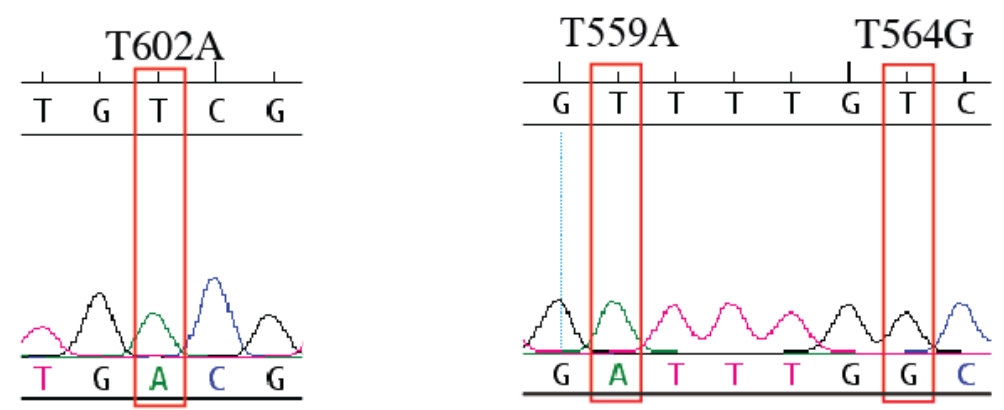

Figure 19: The lethal alleles NC38 and NC109 contained the described mutations

(A) Schematic illustration of the Narf gene structure and the genomic position of the characterized lethality causing mutations. Sequencing analyses of NC38 (B) and NC109 (C) revealed the presence of the described mutations according to Coulthard et al., 2010.

Survival rate assays were performed to address the lethal phase. To be able to distinguish between a homo- and a heterozygous situation, I established fly stocks in which the lethal alleles are kept over GFP-tagged balancer chromosomes (GFP, CyO). As GFP expression was controlled by the twist promoter, the selection according to fluorescence was already possible at embryonic stage and became more striking during larval stage (Figure $20 \mathrm{~A}$ ). Around 100 embryos of each genotype were collected and scored for their survival during development. As control wild type embryos were used, which reach adulthood with a probability of $\sim 75 \%$. Animals expressing the lethal alleles in a heterozygous manner already show a decrease of $\sim 50 \%$ in survival rate from embryonic to larval stage. At this point it has to be mentioned that it is not possible to distinguish between homozygous (GFP, CyO/GFP, CyO) or heterozygous GFP expressing embryos (NC/GFP, CyO) and that the homozygous expression for a balancer chromosome is lethal at embryonic stage (e.g. Meneely, 2014). Thus, the survival rate of heterozygous situations can be considered to be in the same range like wild type. However, homozygous situations showed a clear decrease 
in survival rate and differed in their severeness during development. Hence, the strongest lethal allele was NC37 as it already showed an embryonic lethality. The alleles NC38 and NC109 were larval lethal and animals homozygous for NC70 were even able to survive until adult hood with a probability of $25 \%$.

A

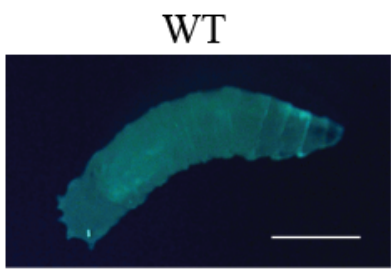
$\mathrm{NC} 37 / \mathrm{Cy} 0, \mathrm{GFP}$

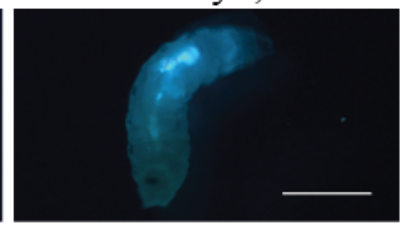

Scale Bar: $1 \mathrm{~mm}$

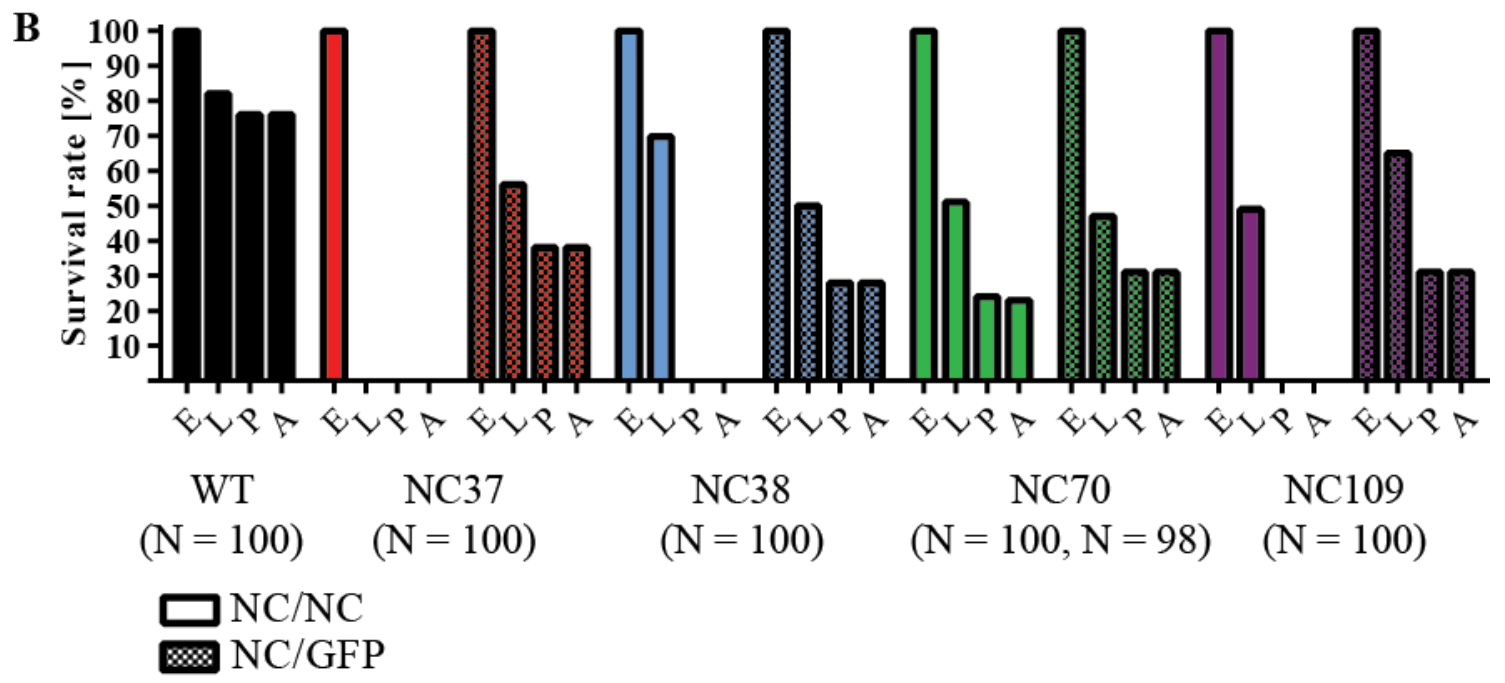

Figure 20: Characterization of different Narf lethal alleles

(A) Fluorescence analysis of wild type and GFP expressing larvae. Wild type larvae showed auto-fluorescence. However, larvae expressing GFP under the control of the twist-promoter were clearly distinguished. (B) Survival rate assays of animals being homozygous or heterozygous for the indicated lethal alleles. Genotype selection was initially based on fluorescence analysis during embryonic stage and survival rate was determined over time.

\subsubsection{The lethal allele NC 38 probably resulted in nonsense-mediated decay}

It was observed that homozygous NC38 animals were able to hatch. However, they died soon during larval stage. In order to characterize the reason of lethality, embryo- and larvae extract were analyzed for Narf protein expression. During embryonic development no differences in protein levels between homo- and heterozygous situations were observed (Figure 21). However, reaching the larval stage was accompanied by a clear decrease in Narf 
protein level. As there was also a protein band shift observable, it was assumed that the detected protein bands represented different isoforms. Thereby, the embryonic expressed Narf might be the isoform PD with a molecular weight of $49.3 \mathrm{kDa}$ (http:/flybase.org/reports/FBgn0262115) and maternally provided, whereas the larval protein might be zygotically expressed and refers to isoform PA (54.5 kDa) or PC (54.1 $\mathrm{kDa}$ ). The premature stop codon in NC38 might led to the nonsense-mediated mRNA decay, thereby causing the reduction of Narf protein, which can be considered as a quasi-null allele.

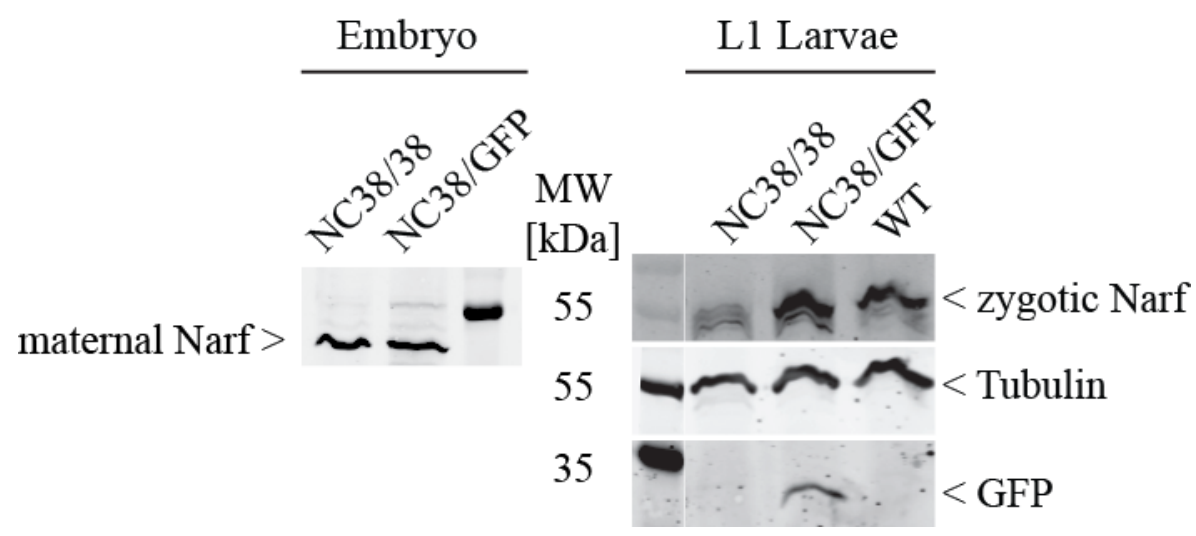

Figure 21: Narf protein level is decreased dramatically in NC38 homozygous larvae.

Western blot analysis of embryo and larvae extract from homozygous or heterozygous situations of the Narf lethal allele NC38. Embryo extract of late embryos did not show a difference in protein level. But, in early L1 stage a decrease in protein level of the homozygous situation was observed in direct comparison to the heterozygous situation and wild type larvae. Furthermore a change in running behavior for Narf was detected in larvae extract, indicating a new zygotically expressed isoform. Tubulin staining was used as loading control and GFP staining was used as a proof for successful genotype selection.

\subsubsection{Genomic transgenes mimicing the human patient situation}

With the aim to identify the organismal effect of the human Narf mutation, I generated genomic constructs of the wild type Drosophila Narf as well as a mutant allele containing an amino acid exchange homologues to the human patient (Narf H/R) (Figure 22). To mimic the heterozygous mutant patient situation it was planned to express the genomic Narf H/R transgenes in NC38 heterozygous flies as it was shown before that this allele comes close to a null allele. Flies that express instead the native Narf in the same genetic background were planned to be used as control. 


\section{Narf human 367 H F V E V L A C 374 Patient allele: H367R \\ (modified to http://www.uniprot.org/align)}

Figure 22: Position of the human patient situation is conserved in Drosophila.

Partial amino acid alignment of the human Narf, referring to the sequence region which contains the human mutation, in comparison to Drosophila Narf. Fully conserved amino acids are marked with an asterisk (*).

In order to establish genomic alleles a touchdown PCR using the oligonucleotides MK44/MK45 was performed on genomic DNA of wild type flies. The PCR product spanning a gene region of 3.992 nucleotides was digested by NheI and BglII and subsequently cloned into the linearized pAttB vector. To introduce the mutation the genomic wild type allele was digested by XhoI and NdeI and the 667 bp fragment was replaced by a synthesized fragment containing the desired mutation. Gene fragment synthesis was done by eurofins including a nucleotide exchange of CAC at position 1323 - 1325 (nucleotides down stream of ATG) against AGA coding for arginine (R) instead of histidine (H). Both constructs were finally site specific integrated into the Drosophila genome using a genomic attP site (25C6) containing fly line. Transgenic flies expressing the genomic Narf wild type allele in wild type background were successfully established. However, it was not possible to establish transgenic flies carrying the mutant allele. Injections of the genomic constructs by Genetivision, using different genomic attP sites (59D3, 68A4), led to similar results showing that the observed lethality of the mutant Narf allele is independent of the integration site. 
A

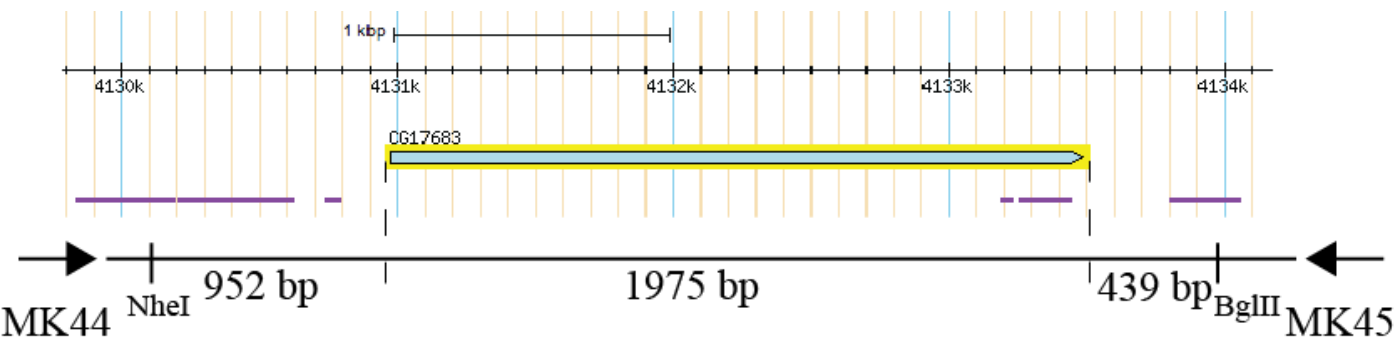

B

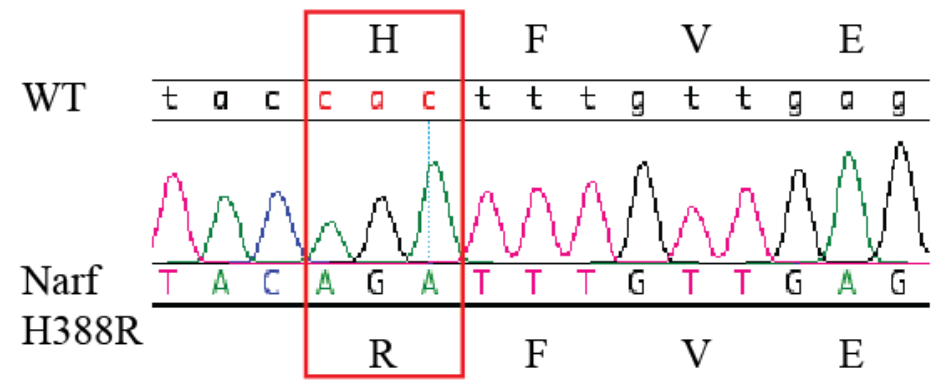

Figure 23: Generation of genomic Narf alleles

(A) Amplified area of the genomic locus used for the generation of genomic Narf alleles. PCR amplification was performed using the oligonucleotides MK44/45 on genomic DNA of wild type flies. Restriction digest by NheI and BglII enabled the cloning into the final donor vector. Schematic illustration was modified according to http://flybase.org/reports/FBgn0262115 (B) Sequencing analysis performed with Seqman confirmed the introduction of the desired mutation by gene synthesis.

\subsubsection{Increase of genomic copies of Narf might positively affect viability}

Since it was not possible to establish a genomic Narf H/R allele, the question arose if expression of an increased number of genomic Narf copies affects viability. Three differently inserted transgenic lines for the Narf wild type allele were established (25C6-2L, 59D3-2R, 68A4-3L), in none of them an effect on viability and fertility has been observed. To study this in more detail survival assays and lifespan analysis have been performed using one transgenic line which was integrated on the second chromosome. To be able to distinguish between homo- and heterozygous flies, expressing three or four genomic copies of Narf, the transgenic flies were kept over a GFP-tagged balancer chromosome (GFP, CyO). Survival rate analysis during development showed, that the expression of an increased number of genomic copies is not negatively affecting viability (Figure $24 \mathrm{~A}$ ). However, compared to wild type embryos Narf overexpressing animals even show a higher survival rate. As it is not possible to distinguish between homozygous (GFP, CyO/GFP, CyO) or heterozygous GFP expressing embryos (genomic Narf/GFP, CyO) around 25\% of the 3x 
genomic Narf expressing embryos (Figure 24 A, green) will die before reaching larval stage, due to a homozygous expression of the balancer chromosome. Thus, the survival rate of both overexpressing situations seems to be increased compared to wild type embryos.

Furthermore no decrease on lifespan was detected compared to wild type flies (Figure 24 B). Thereby, it also seems that Narf overexpressing flies show an increase in lifespan dependent on Narf copy number. At this point it has to be mentioned that the used control, which was the originally injection strain (BL\#25709-25C6), had the most similar genetic background but already showed a shortening on lifespan compared to wild type flies. Thus it was not clear if the difference in lifespan might be an inbreeding effect within control flies and a hybrid effect on Narf expressing flies or dependent of Narf.

In order to verify the effect of gene dose on life span, flies containing genomic transgenes on different chromosomes were crossed with each other. However, the upper limit was the expression of four genomic copies, since genomic Narf integrated on the third chromosome was homozygous lethal perhaps due to integration site. 
A
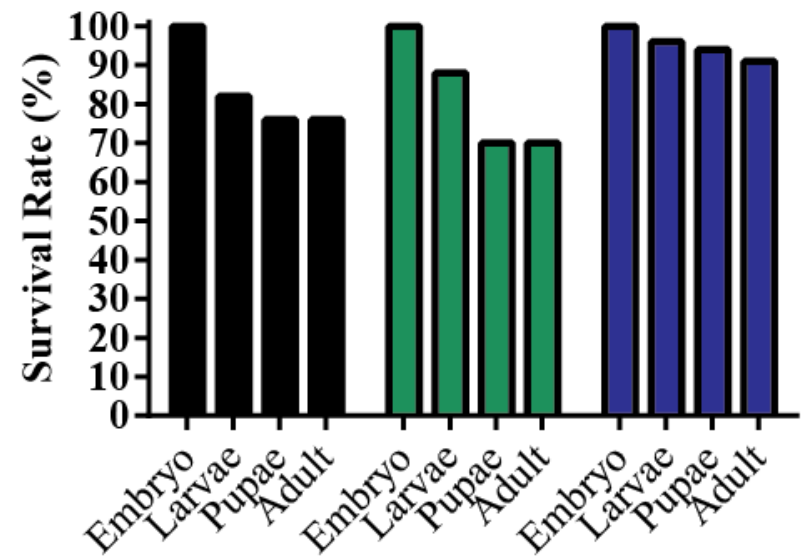

Developmental Stages

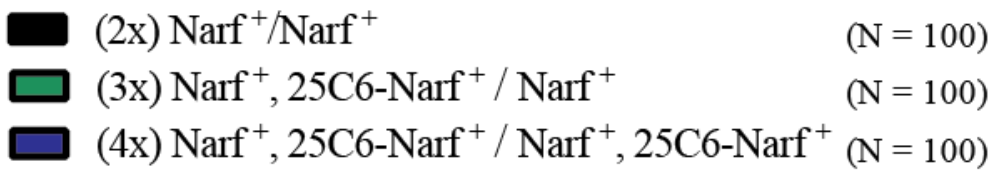

B

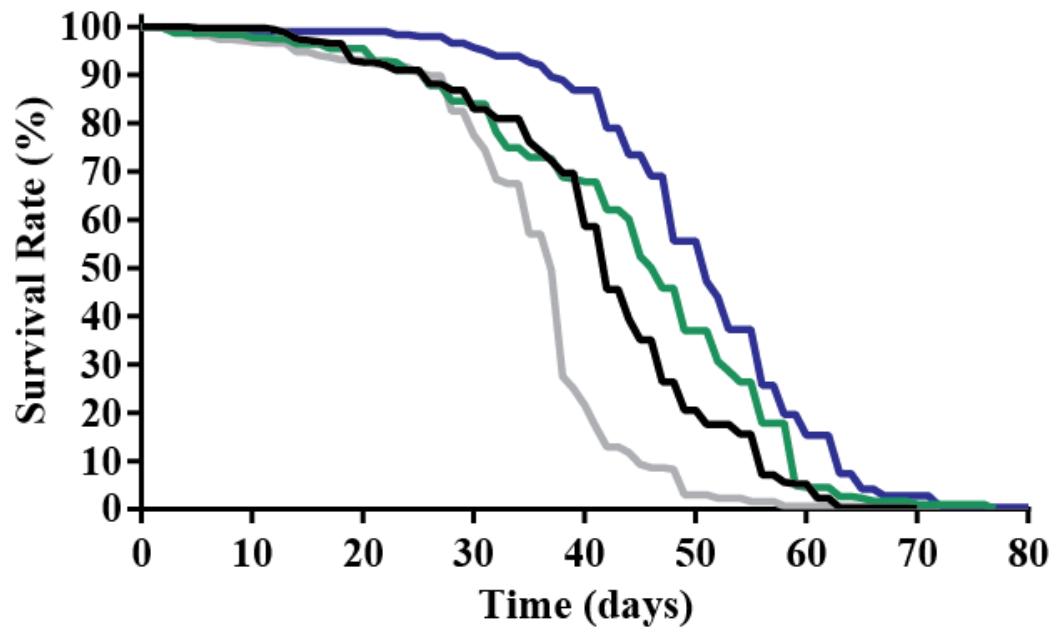

- (2x) $\mathrm{Narf}^{+} / \mathrm{Narf}^{+}$ (2x) $\mathrm{Narf}^{+}, 25 \mathrm{C} 6 / \mathrm{Narf}^{+}, 25 \mathrm{C} 6$

$(\mathrm{N}=315,4$ experiments $)$

(3x) Narf $^{+}, 25 \mathrm{C} 6-\mathrm{Narf}^{+} / \mathrm{Narf}^{+}$

$(\mathrm{N}=400,4$ experiments)

( $\mathrm{N}=311,4$ experiments)

- (4x) Narf $^{+}, 25 \mathrm{C} 6-\mathrm{Narf}^{+} / \mathrm{Narf}^{+}, 25 \mathrm{C} 6-\mathrm{Narf}^{+} \quad(\mathrm{N}=297,4$ experiments $)$

Figure 24: Overexpression of genomic Narf increased survival rate

(A) Survival rate assay of animals expressing three $(3 \mathrm{x})$ or four (4x) copies of genomic Narf compared to wild type embryos with naturally two genomic Narf copies $(2 \mathrm{x})$. Narf overexpression had no negative effects on survival rate during development. (B) Survival rate analyses during adulthood showed that an overexpression of genomic Narf alleles prolonged lifespan (log-rank test: Ctrl vs. WT p $<1.0{ }^{*} 10^{-10}$, WT vs. 3x Narf $p=1.6$ ${ }^{*} 10^{-5}$, WT vs. $4 \mathrm{x}$ Narf $\mathrm{p}<1.0^{*} 10^{-10}$, 3x Narf vs. 4x Narf $\mathrm{p}=8.1^{*} 10^{-6}$ ). Statistical analyses were performed by OASIS, illustration was done using GraphPad Prism6. 


\subsubsection{Ubiquitous overexpression of Narf had beneficial effects on lifespan}

It was observed that an increase of the genomic copy numbers of Narf prolonged lifespan (Figure 24). Furthermore, it was shown that a higher improvement of survival rate can be achieved by expressing four instead of three genomic copies. To further validate these data a Narf overexpressing situations was accomplished using the UAS/GAl4 system. As lifespan seemed to be dependent on Narf expression strength, different ubiquitous drivelines (actin- (GS), armadillo and tubulin-Gal4) were tested for their effect on Narf protein expression level in adult males (Supplement Figure 4). A control experiment was performed with a transgenic UAS-GFP fly line. It is shown that highest GFP expression was achieved using the actin GS or tubulin driver line. However, highest Narf expression was detected in case of tubulin-Gal4. Thus, lifespan analyses were performed overexpressing both Narf alleles (wild type and H/R mutant) controlled by tubulin-Gal4. To prevent differences in lifespan due to hybrid effects and to provide a control situation with the most similar genetic background, the originally used injection strain (BL\#25709-25C6) was crossed against the driver line. Consistent to the before mentioned data a prolongation in lifespan was observed. Furthermore, comparable lifespan curves were detected for both Narf alleles. To summarize, it was shown that Narf overexpression, starting during early development, had beneficial effects on organismal level, reasoning a role of Narf in aging.

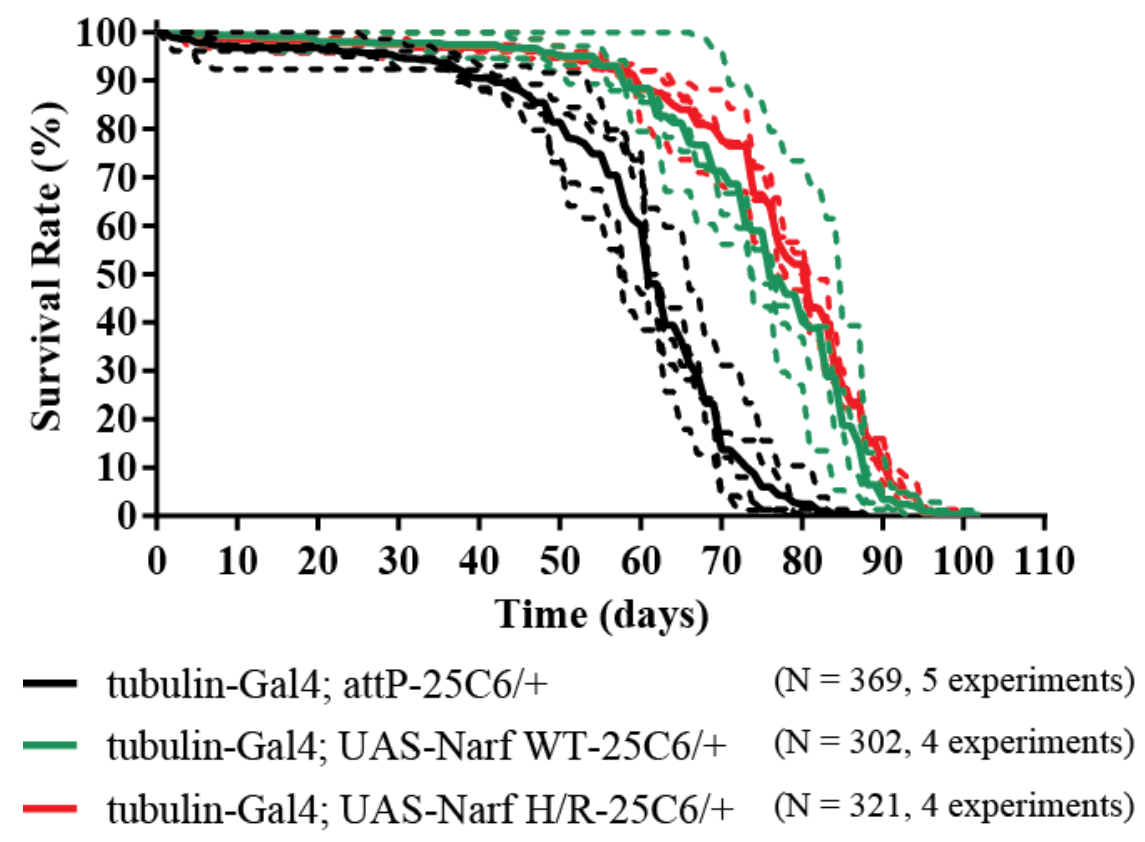

Figure 25: Ubiquitous overexpression of Narf prolonged Drosophila lifespan

Lifespan analyses of Narf overexpressing flies (green: wild type allele, red: mutant H/R allele) compared to control flies (black). Overexpression was controlled by tubulin-Gal4 a ubiquitous driver line. Survival rate 
analyses were performed with a minimum of four replicates (dashed lines) each with $~ 75$ males. The mean value was taken (solid lines) and used for statistical analyses. A ubiquitous up regulation of Narf significantly increased lifespan (log rank test: Ctrl vs. OE Narf WT p $<1 * 10^{-10}$, Ctrl vs. OE Narf H/R p $<1 * 10^{-10}$, OE Narf WT vs. OE Narf H/R p = 0.0033).

\subsubsection{Using the CRISPR approach for generating a Narf null allele}

In order to study Narf function in a clear genomic background it was planned to generate a Narf null allele using the CRISPR/Cas9 approach. Thereby targeted chromosomal double strand breaks (DSB) are induced using the microbial Cas9 nuclease protein. Site directed specificity is achieved by locating the Cas9 protein via single guided RNAs (sgRNA) which consist of 20 nucleotides excluding the protospacer-adjacent motif (PAM) (Jinek et al 2012). DNA cleavage results in activation of the DSB repair machinery which will remove DNA damage by the non-homologous end joining (NHEJ) pathway or the homology-directed repair (HDR) pathway (reviewed in Lieber et al., 2010). The second repair mechanism is based on the insertion of a homology DNA template, enabling gene deletions by replacing the gene locus with exogenous sequences. In this study the pHDDsRed-attP vector was used as a tool to deplete the genomic locus of Narf in exchange with a dsRed selection marker, loxP sites and an attPsite, allowing site specific recombination after introduction within the gene locus (Gratz et al., 2014). A germ-line specific expression of the Cas-9 protein is furthermore preventing lethality in case of essential genes. Narf specific sgRNAs were identified by the CRISPR Optimal Target Finder (http://flycrispr.molbio.wisc.edu), generated by oligonucleotides annealing and cloned into the BbsI linearized pBFvU6.2 and U6.2B vectors. To enable HDR two homology arms of around $1 \mathrm{~kb}$ (Beumer et al., 2013, Gratz et al., 2014) were amplified and successively cloned into the linearized pHD-DsRed-attP vector. Thereby adjacent areas to the genomic locus of Narf were chosen which lied outside of repetitive sequences (Figure 26 A, purple lines). Subsequent injection in Cas9 expressing embryos was performed by BestGene Inc. However, out of 55 GO adults no RFP positive G1 fly was established. A fail in null allele generation might be reasoned to the heterochromatic position of Narf next to the centromere or the large gene spanning region of $\sim 5.3 \mathrm{~kb}$, decreasing efficiency in repair mechanism. In order to still get a null allele of Narf, the all-inclusive service from inDroso was used. In this case an attP site will be introduced by replacing the gene area from the 5 'UTR till 368 nucleotides after the 3`UTR (position 4130968 to 4133852). Once a conditional null allele is established a tool is generated which enables the study of a multitude of Narf situations 
(e.g. knock-out situation, GFP-transgenes or analyses of a genomic and mutant Narf H/R allele in a clonal flip-out system (Figure 26 B)).

A

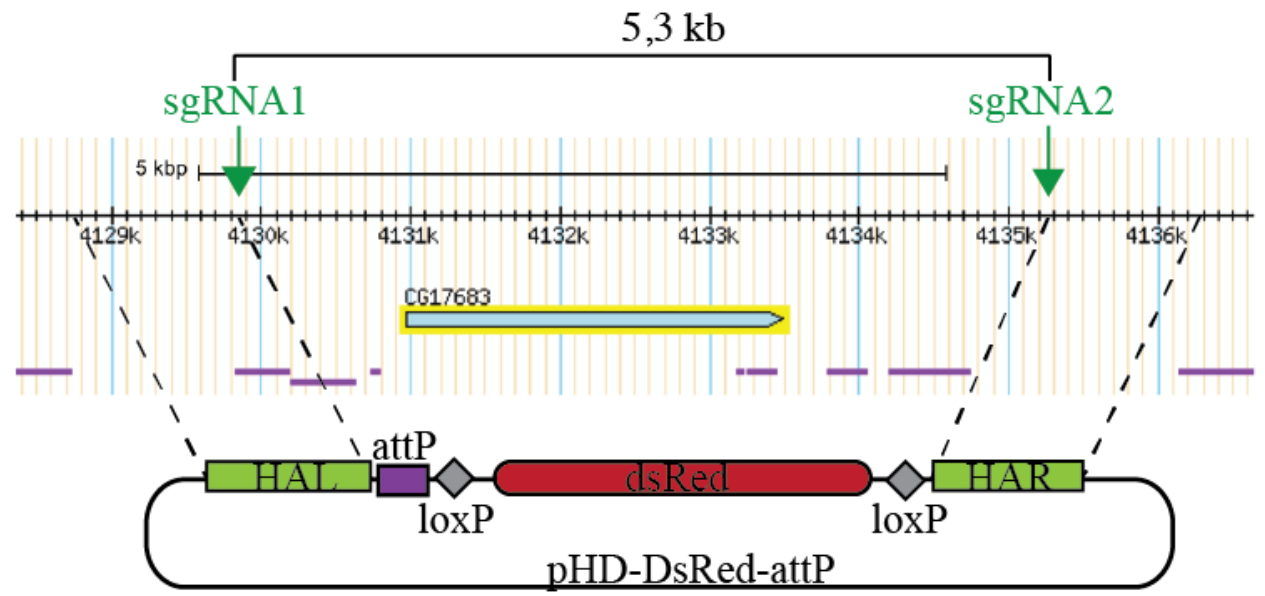

(1) pHD-DsRed-attP

HDR

(2)

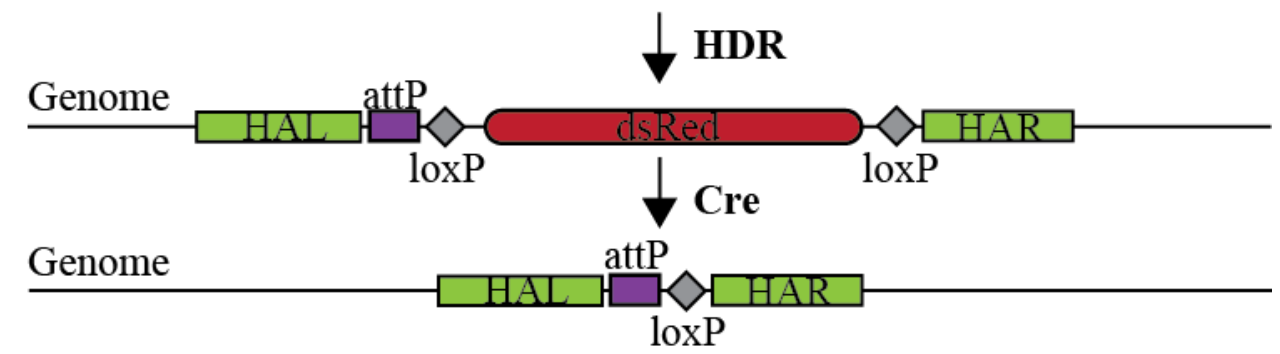

B

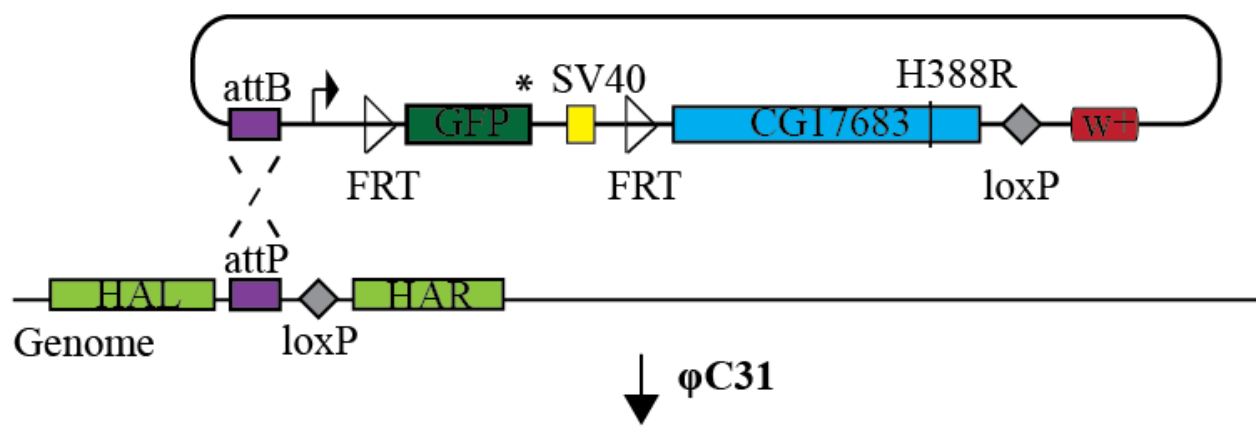

(3)

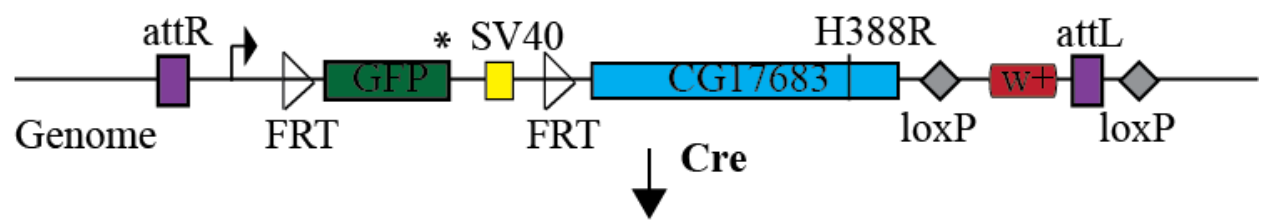

(4)

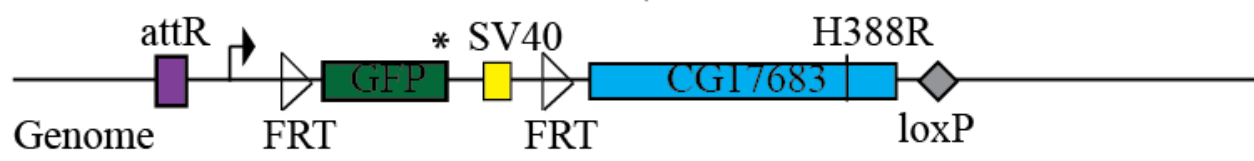

(5)

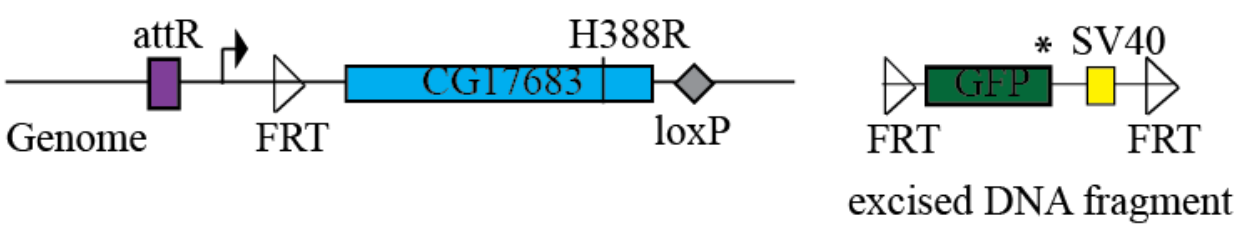

Flp 
Figure 26: Generation of a conditional Narf null allele by CRISPR/Cas9

(A) Single guided RNAs (sgRNAs) are used to target the Cas9 nuclease protein and induce site specific chromosomal double strand breaks (DSBs). Upon DNA damage the DSB repair machinery will be activated. By providing an exogenous vector sequence, including two $1 \mathrm{~kb}$ homologues gene regions (HAL-homology arm left, HAR-homology arm right), homology directed repair (HDR) will take place (1). Thus, a null allele is generated by replacement of the genomic locus. Successful generation of transgenic flies can be detected by the introduced selection marker dsRed, which can be later removed by Cre-loxP recombination (2). (B) The introduction of a genomic attP site enables site specific integration by using the $\varphi \mathrm{C} 31$ integrase and makes the null allele conditional. To mimic the heterozygous human patient situation it is planned to express a genomic Narf (H/R) allele in a clonal and negatively GFP-marked manner. Potential lethal effects will be prevented by a tissue specific expression. In order to do this an attB vector will be site specifically integrated (3) carrying the coding sequences for GFP and the desired Narf allele. A successful integration will destroy the attP site which will be detected by the expression of the mini white $\left(\mathrm{w}^{+}\right)$selection marker. Excessive vector sequences will be removed by Cre-loxP recombination, enabled by the introduction of a second loxP site and recognized by the loss of the mini white $\left(\mathrm{w}^{+}\right)$selection marker (4). Thus, the genomic Narf promoter will control GFP expression up to the stop codon $\left(^{*}\right)$ and followed by a SV40 terminator signal. As these sequences will be flanked by two FRT (flippase recognition target) -sites the expression of a flippase recombinase (FLP) will be able to remove this cassette in a tissue specific manner (5). This approach will allow the heterozygous expression of a genomic and mutant Narf $H / R$ allele. Narf expressing cells will be negatively marked by the loss of GFP expression.

\subsubsection{The GeneSwitch Gal4 system was used to muscle specifically express GFP in a dose dependent manner}

Another possibility to study protein function is the usage of the GeneSwitch-Gal4 system. This approach enables a spatial and temporal specific expression of transgenes within Drosophila. The basic principle behind is that the GeneSwitch-Gal4 protein becomes only active in the presence of the mifepristone (RU 486, hereinafter referred to shortly as RU) activator. Spatial expression is given by a tissue specific enhancer, which drives Gal4 gene expression (Figure $27 \mathrm{~A}$ ). The big advantage of this system is the same genetic background of induced and non-induced flies, thereby providing a better control.

In order to know the right working concentration range of RU, I checked the effect of target gene expression under different RU concentrations. Therefore, an UAS-GFP transgene was muscle specifically expressed using the MHC-GS Gal4 (myosin heavy chain) driver line. The GFP level was then used as readout for gene expression level. Immunofluorescence analyses and western blot analyses showed that the GFP protein was expressed within thoracal muscle tissue after 10 days of induction (Figure 27 B, C). Furthermore it was shown 
that this expression occurs in a dose dependent manner, controlled by distinct RU-concentrations (Figure 27 B). A dose-response relationship, based on the measured mean grey value of the detected GFP-signal and a control-signal, is shown in Figure 27 D. The half maximal effective concentration (EC50) within a range of $2-200 \mu \mathrm{M}$ of RU was found to be at $50 \mu \mathrm{M}$. 


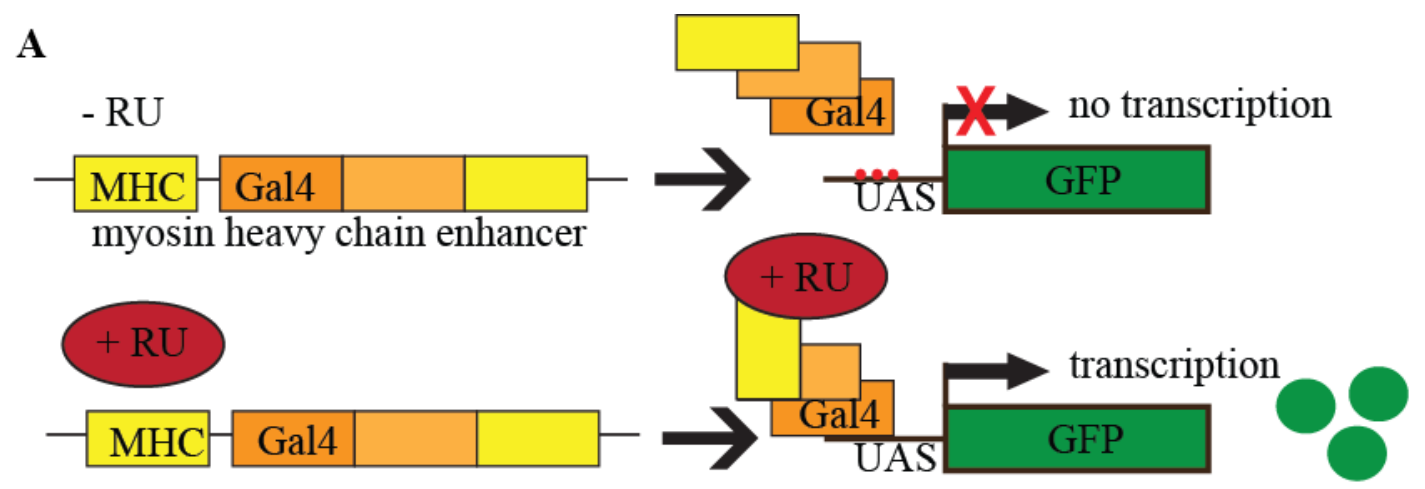

(modified to Nicholson et. al., 2008)

\section{B}

Live-Imaging

$100 \mu \mathrm{M}$ RU

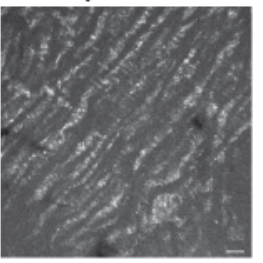

Scale Bar: $10 \mu \mathrm{m}$
C

$\mathrm{kDa}$<smiles>C1=[Co]C1</smiles><smiles>[C]1[CH]C=C1</smiles><smiles>C1CCCC1</smiles><smiles>[SiH3]</smiles>

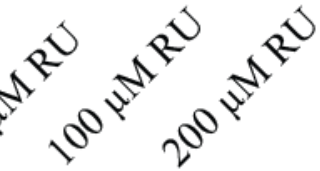

D Nonlin fit of log-dose vs response

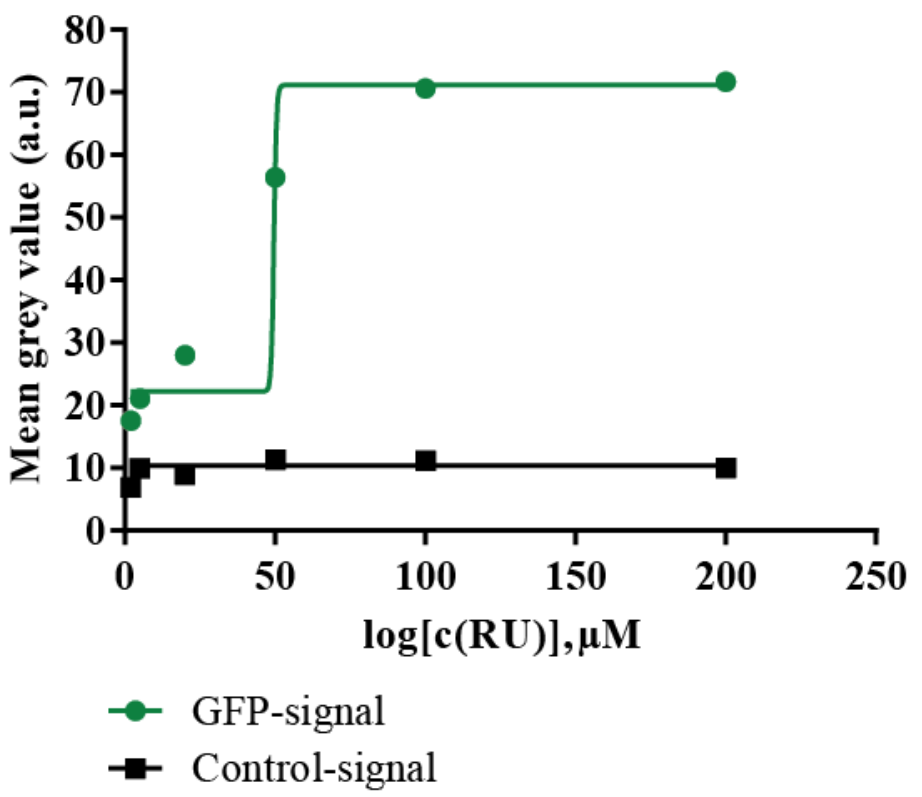

Figure 27: The GeneSwitch system enables spatial and dose-dependent gene expression

(A) Schematic illustration of the basic mechanism of the GeneSwitch system. Spatial and temporal gene expression is controlled by a tissue specific driver (e.g. MHC) which control Gal-4 protein expression. Transcriptional activation of the Gal-4 protein is achieved in the presence of the activator mifepristone (RU486) 
which enables expression of an UAS-transgene (e.g. UAS-GFP). (B) Live-imaging of thoracal expressed GFP controlled by the MHC driver line. Induction was performed with $100 \mu \mathrm{M}$ of RU for 10 days. (C) Western blot analyses of thorax samples of GFP expressing flies controlled by the MHC enhancer. Induction was done as indicated with different concentrations of RU for 10 days. Antibody staining was performed against GFP and $\alpha$-tubulin as control. An unspecific signal generated by the GFP antibody was used as an additional controlsignal for subsequent mean grey value quantification. (D) Logarithmic dose-response relationship, based on the measured mean grey value of the detected GFP-signal and the control-signal. The $\mathrm{EC}_{50}$ concentration was calculated to be at $50 \mu \mathrm{M}$ within a range of $2-200 \mu \mathrm{M}$ of RU.

\subsubsection{The GeneSwitch system was used to muscle specifically overexpress Narf}

In order to use the GeneSwitch system to study Narf-protein function, I generated transgenic flies containing the wild type Narf and the mutant version driven by UAS. Therefore, the cDNA clone RE37350, provided by the Berkeley Drosophila Genome Project (BDGP), was used. To study the effect of the mutant Narf allele, the H388R mutation was introduced via site directed mutagenesis. Subsequent sequencing analysis confirmed the success. The cDNA fragments of the wild type and the mutant situation were subsequently cloned into the pUASt-attB vector via NotI/BamHI restriction followed by a blunt end ligation into the NotI/XhoI linearized donor vector. Both constructs were finally site specifically integrated using embryos containing a genomic attP site and transgenic flies were successfully established.

Next, I checked if it was possible to overexpress Narf in the muscle using the GeneSwitch system with two different concentrations of RU (20 $\mu \mathrm{M}, 200 \mu \mathrm{M})$. Western blot analysis of thorax samples confirmed that Narf was indeed overexpressed after 10 days of induction compared to non-induced flies (Figure 28). However, a dose dependency was not as clear as it was seen for GFP overexpression (Figure 27 C).

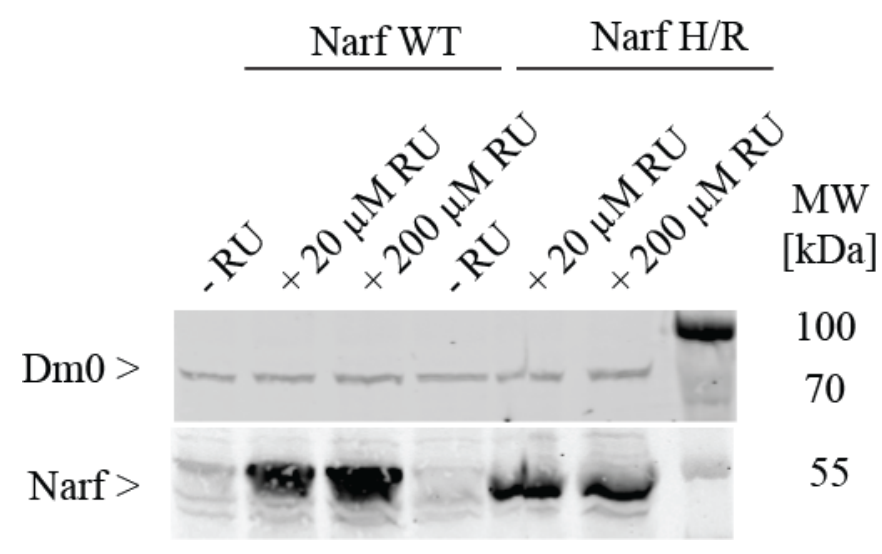


Figure 28: The GeneSwitch Gal4 system enabled Narf overexpression

Western blot analysis of thorax samples of Narf overexpressing flies controlled by the MHC enhancer. Induction was done with $20 \mu \mathrm{M}$ and $200 \mu \mathrm{M}$ of RU for 10 days. Non-induced flies were used as control. Antibody staining was performed against Narf and lamin Dm0 as control. Both RU concentrations induced a Narf overexpression. However a dose dependent difference in gene expression was not detected.

\subsubsection{Overexpression of Narf increased nuclear size in muscle cells}

One of the hallmarks of progeria patient cells is an abnormal nuclear structure. Next, I examined the effect of Narf overexpression on nuclear morphology. An immunofluorescence analysis of thorax samples, using an antibody staining for lamin Dm0 as nuclear marker, was performed. After 10 days of induction an increase in nuclear size has been observed in Narf overexpressing flies compared to non-induced control flies (Figure 29). Furthermore, quantification of the nulei area showed that the overexpression of the mutant Narf allele seems to be more severe (Figure 29 D). However, changes in nuclear positioning or muscle structure have not been observed. To further validate if RU alone is inducing nuclear size changes, wild type flies crossed with the MHC-Gal4 driver line were treated with $200 \mu \mathrm{M}$ RU. As the quantification shows RU alone is indeed affecting nuclear size. However, nuclei in a Narf overexpressed situation are still significantly larger, suggesting an additive effect of Narf and RU in these situations. 
A MHC-GS Gal4 Narf WT - RU (Control)

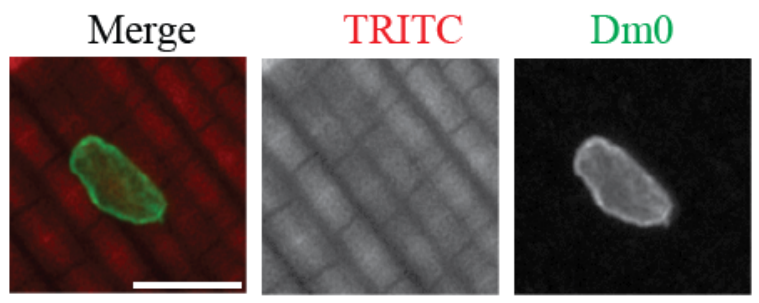

B MHC-GS Gal4 Narf WT + $200 \mu \mathrm{M}$ RU

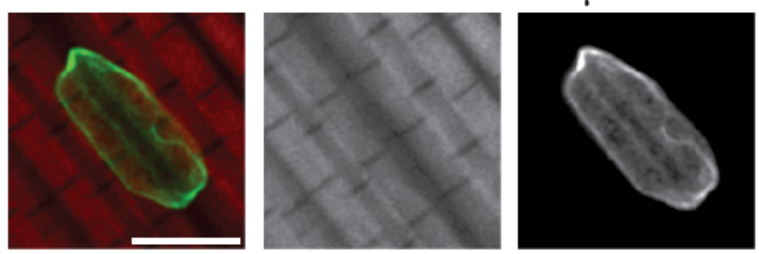

C MHC-GS Gal4 Narf H/R + $200 \mu \mathrm{M}$ RU

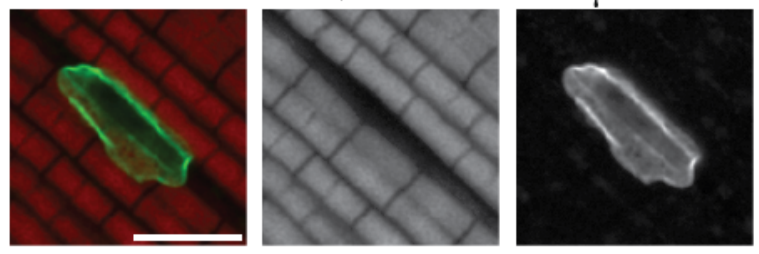

Scale Bar: $5 \mu \mathrm{m}$

D

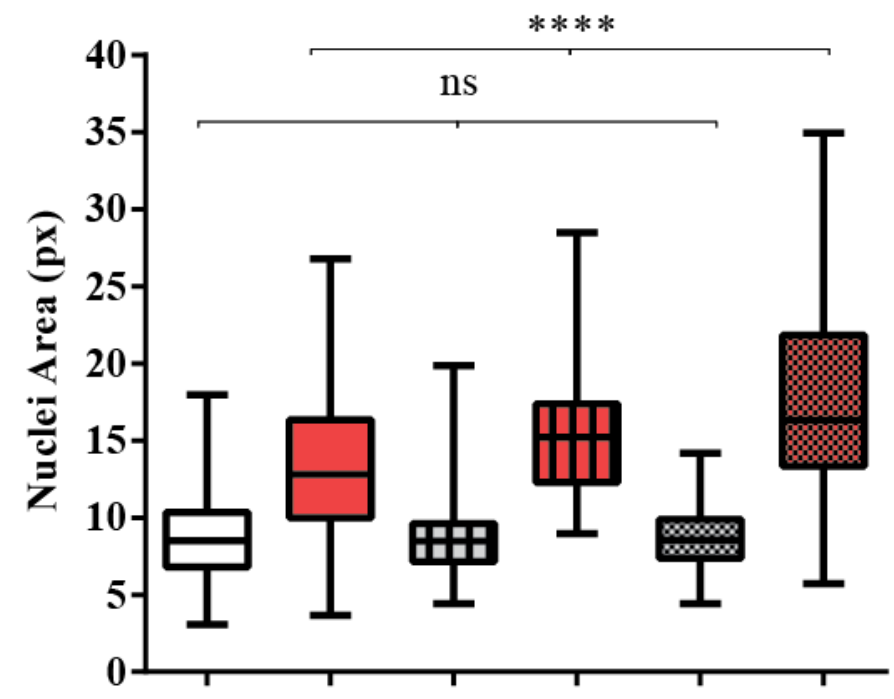

Control $\left[\begin{array}{ll}\square-\text { RU, MHC-GS Gal4; WT } & \text { (N=5/310 nuclei) } \\ \square+200 \mu M ~ R U & (\mathrm{~N}=5 / 162 \text { nuclei) }\end{array}\right.$

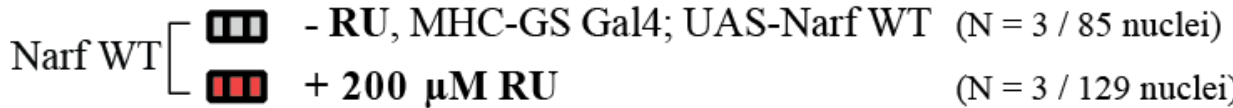

Narf H/R - RU, MHC-GS Gal4; UAS-Narf H/R (N = 3 / 100 nuclei)

$+200 \mu \mathrm{M} R$

$(\mathrm{N}=3 / 166$ nuclei $)$ 
Figure 29: Narf overexpression affected nuclear size

(A-C) Immunofluorescence analyses of thorax samples. Overexpression of the wild type (B) or the mutant Narf allele (C) was induced using $200 \mu \mathrm{M}$ of RU for 10 days. Non-induced flies were used as control (A). Nuclei were stained with lamin Dm0 and muscle tissue was marked using TRITC phalloidin. (D) Quantification of the nuclei area. Wild type flies crossed with the MHC enhancer and treated with $200 \mu \mathrm{M}$ of RU showed an increase in nuclear size verifying that RU alone has an effect on nuclear size compared to non-induced situations $\left(\mathrm{Ctrl}^{-\mathrm{RU}}\right.$ vs. $\mathrm{Ctrl}^{+\mathrm{RU}} \mathrm{p}=3.19^{*} 10^{-21}$ ). Overexpression of the Narf alleles induced by $200 \mu \mathrm{M}$ of $\mathrm{RU}$ significantly increased nuclear size compared to RU treated control flies (Ctrl ${ }^{+R U}$ vs. Narf $\mathrm{WT}^{+\mathrm{RU}}: \mathrm{p}=9.66$ $* 10^{-6}, \mathrm{Ctrl}^{+\mathrm{RU}}$ vs. Narf H/R $\left.\mathrm{R}^{+\mathrm{RU}}: \mathrm{p}=4.74 * 10^{-16}\right)$. Overexpression of the mutant Narf allele had stronger effects on nuclear size than overexpression of the wild type allele (Narf WT vs. Narf H/R: $\mathrm{p}=1.15^{*} 10^{-6}$ ). Quantification was done manually using ImageJ and statistical significance was calculated using unpaired $t$ test $(* * * * \mathrm{p}<0.0001)$.

\subsubsection{Muscle specific overexpression of Narf affected negative geotaxis behavior}

After finding that Narf overexpression is inducing nuclear shape changes, I wondered if this might be associated with muscle weakness. In order to study this a negative geotaxis experiment was performed. Negative geotaxis is a natural and age dependent climbing behavior of Drosophila characterized by a negative response to gravity. It was already shown that a muscle specific overexpressing of Kuk or Lamin B results in an early decline in this climbing ability (Brandt et al., 2008). To validate if a muscle specific overexpression of the wild type and the mutant Narf allele leads to a similar phenotype negative geotaxis assays with a maximal duration of eight weeks were performed. Non-induced flies with the same genetic background were used as controls. RU induction was done for one week before measurements of the climbing high were started. The mean climbing high of the first measurements was set to $100 \%$. In both control situations a very similar age dependent decline in mobility was visible. Induced flies showed a clear decrease in negative geotaxis compared to control flies independent of the overexpressed Narf alleles (wild type or H/R) and the used RU concentration (Figure $30 \mathrm{~A}, \mathrm{~B}$ ). An explanation that the observed effect is not becoming more severe with an increased RU concentration can be given by the very similar protein expression of Narf using $20 \mu \mathrm{M}$ or $200 \mu \mathrm{M}$ of RU (Figure 28). However, as it was seen before that RU alone is indeed affecting nuclear size this experiment has to be complemented by an additional control to rule out that RU alone is sufficient to induce the observed effects. This experiment was also started but could not be finished due to seasonal temperature differences which caused a change in Drosophila behavior. However, it was 
shown that nuclear shape changes are dependent on Narf expression, suggesting that this will be also true for negative geotaxis behavior.

A MHC GS Gal4; UAS-Narf WT

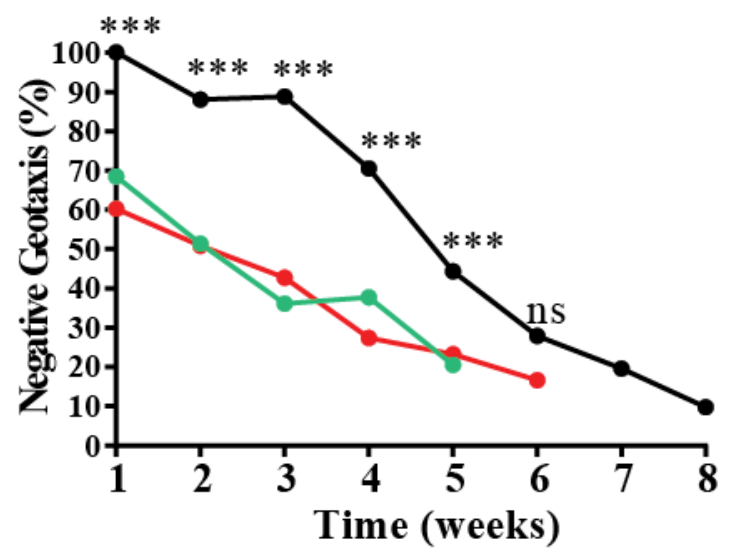

- - - RU (control)

$-\quad+20 \mu \mathrm{M} \mathrm{RU}$

$\multimap+200 \mu \mathrm{MRU}$

B MHC GS Gal4; UAS-Narf H/R

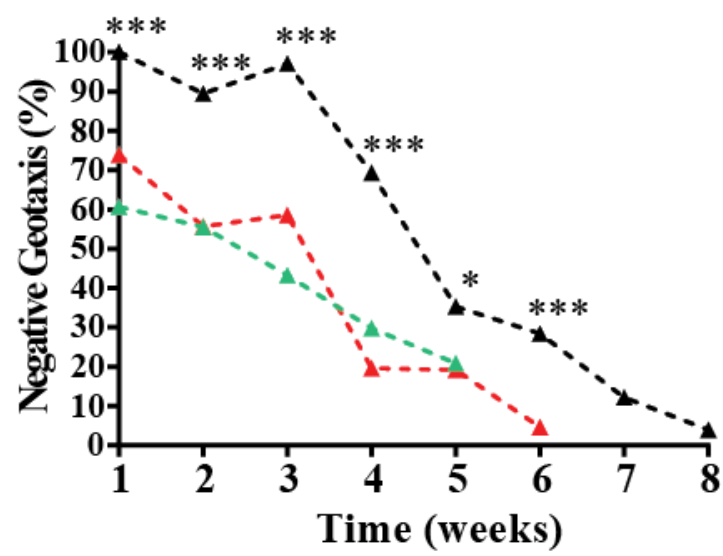

-^. - RU (control)

$-\ldots \cdot+20 \mu \mathrm{MRU}$

-« · + $200 \mu \mathrm{M} \mathrm{RU}$

C

MHC GS Gal4; UAS-Narf

WT vs. H/R

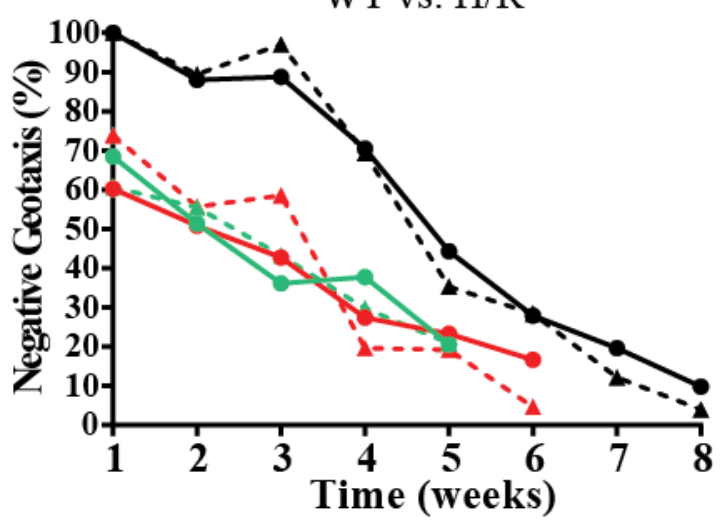


Figure 30: Narf overexpression might affect negative geotaxis behavior

(A-B) Negative geotaxis experiments of Narf overexpressing flies induced by either $20 \mu \mathrm{M}$ (green) or $200 \mu \mathrm{M}$ of RU (red). Measurements were started after one week of induction and performed with at least 10 and maximum 25 males dependent on survival rate over time. Mean climbing high of one week old non-induced control flies was set to $100 \%$. For both situations a clear decrease in climbing high is observed independent of the used RU concentration. (C) Direct comparison of the mean climbing high in flies overexpressing the wild type and the mutant Narf allele. No significant changes between the wild type and the mutant allele were detected using $20 \mu \mathrm{M}$ of RU (green circle/triangle). Low significant changes were detected for $200 \mu \mathrm{M}$ (red circle/triangle) after week 3 and 6 (week 3: $\mathrm{WT}^{+200}$ vs. $\mathrm{H} / \mathrm{R}^{+200} \mathrm{p}=0.0279$, week 6: $\mathrm{p}=0.0389$ ). Statistical significance was calculated using unpaired $t$-test (ns $\mathrm{p}>0.05$, ${ }^{*} \mathrm{p}<0.05$, ** $\mathrm{p}<0.01,{ }^{* * *} \mathrm{p}<0.001$ ).

\subsubsection{Muscle specific overexpression of Narf affected Drosophila lifespan}

Negative geotaxis experiments were performed over a maximal time period of 8 weeks. But, the analyses of induced flies had to be stopped 2-3 weeks earlier compared to control flies due to a lower survival rate. This observation led me hypothesize that an overexpression of Narf leads to a shortening in lifespan. However, to exclude that RU induction itself is responsible for a decrease in survival rate a control experiment using wild type flies and $200 \mu \mathrm{M}$ of RU was performed. The analyses were done in four replicates each with around 75 male due to a high level of intraspecific variations in Drosophila lifespan. It is shown in Figure 31 that RU alone is not affecting lifespan, suggesting that Narf overexpression might be associated with a decrease in lifespan. 


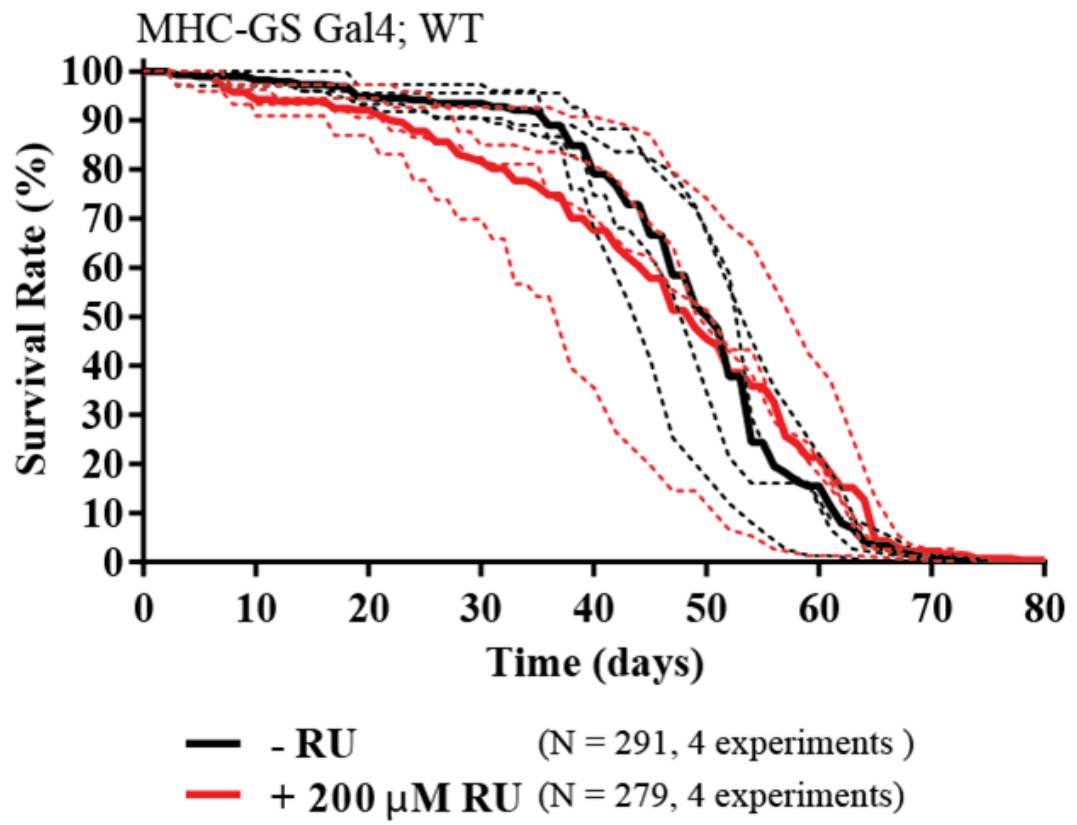

Figure 31: RU468 treatment did not affect Drosophila lifespan

Lifespan analyses of wild type flies treated with $200 \mu \mathrm{M}$ of RU (red) compared to non-induced control flies (black). Survival rate analyses were performed in four replicates (dashed line) each with $\sim 70$ males. The mean value was taken (solid line) and used for statistical analyses which was performed using OASIS an online application tool for lifespan analysis, log-rank test $\mathrm{p}=0.2265$ ( $\mathrm{p}>0.05$, not significant).

In order to provide a proof that Narf overexpression affects survival rate during adulthood lifespan analyses with Narf overexpressing flies were performed. To analyze if an overexpression of the mutant Narf allele might further affect lifespan both alleles were tested in parallel. For both alleles a clear decrease in lifespan upon Narf overexpression compared to non-induced control flies was observed (Figure 32). This effect seems to be dosedependent as lifespan shortening correlates with the RU concentration. A quite interesting observation was that the lifespans of the wild type and the mutant Narf allele behaved quite similar if induced with $20 \mu \mathrm{M}$ of RU. An induction with $200 \mu \mathrm{M}$ instead seems to cause more severe effects for the mutant Narf H/R allele, indicating a gain-of function for the mutant allele. 
A MHC-GS Gal4; UAS-Narf WT

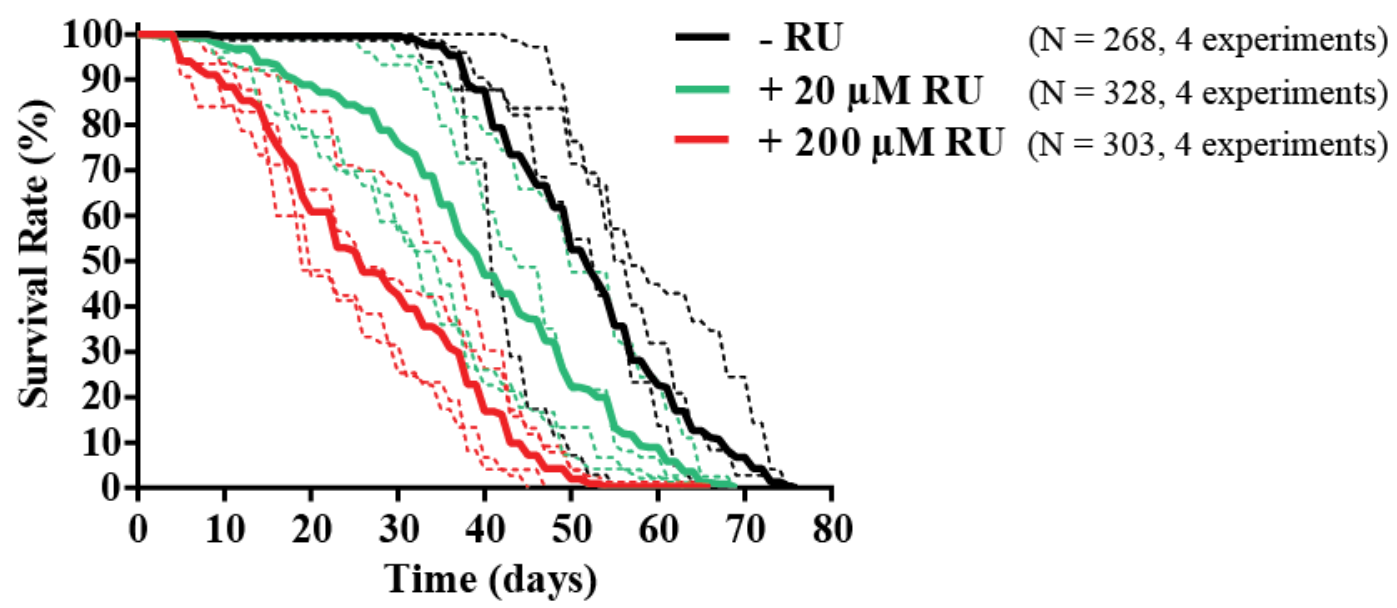

B

MHC-GS Gal4; UAS-Narf H/R

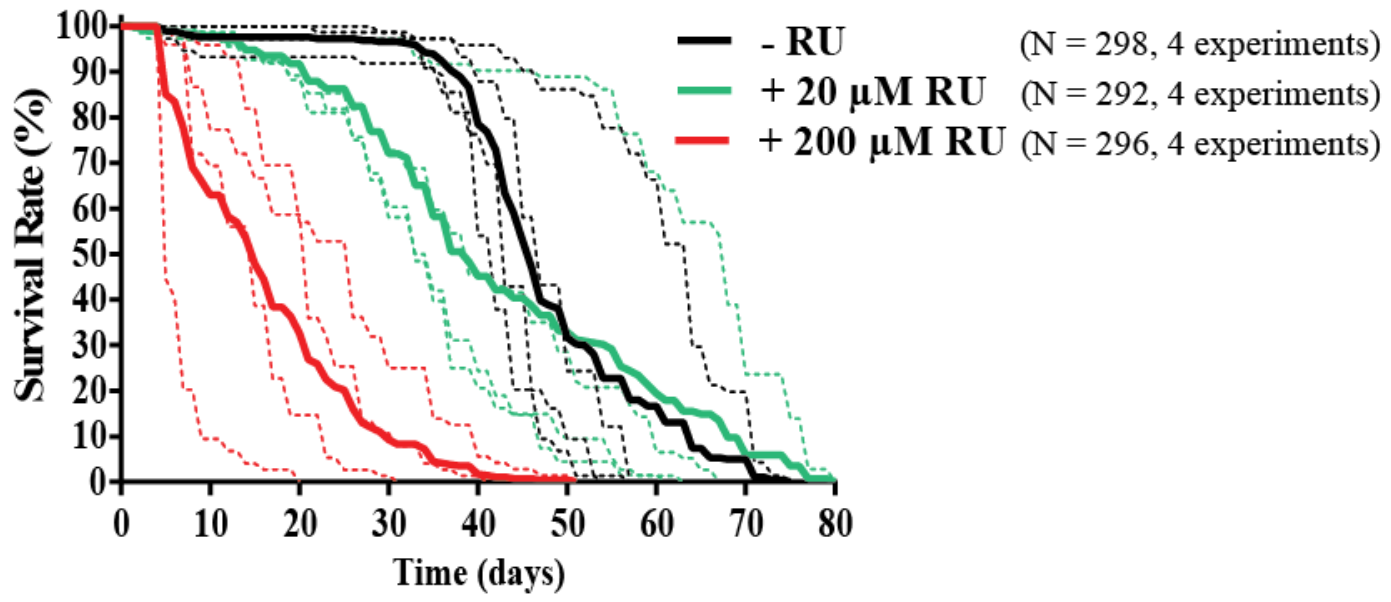

C

MHC-GS Gal4; UAS-Narf WT vs. H/R

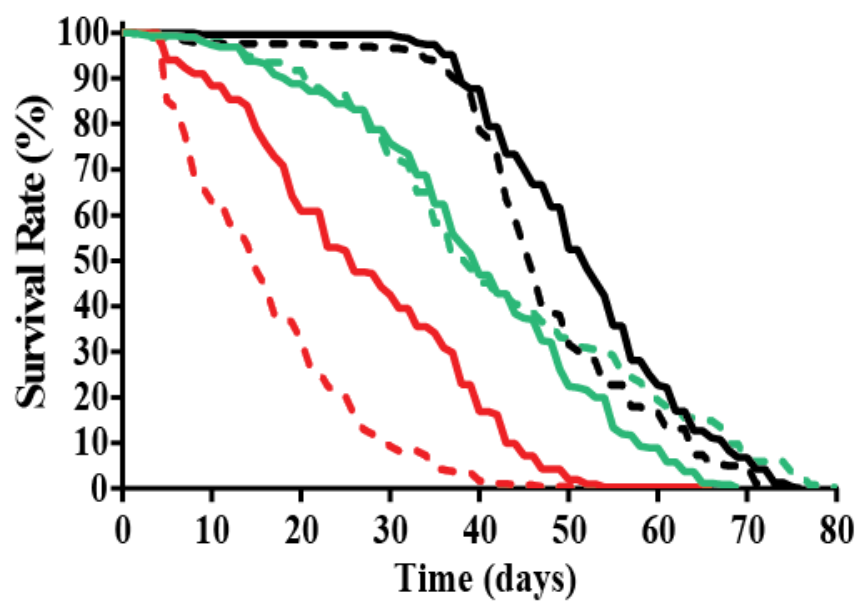

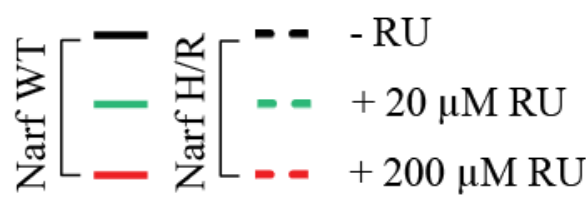


Figure 32: Narf overexpression during adulthood affected Drosophila lifespan

(A-B) Lifespan analyses of Narf overexpressing flies (A - wild type allele, B - mutant H/R allele) treated with $20 \mu \mathrm{M}$ (green) and $200 \mu \mathrm{M}$ of RU (red) compared to non-induced control flies (black). Survival rate analyses were performed in four replicates (thin lines) each with $\sim 75$ males. The mean value was taken (thick lines) and used for statistical analyses. For both Narf overexpressing situations dose-dependent decrease in survival rate was detected compared to non-induced control flies (log-rank test: $\mathrm{WT}^{-\mathrm{RU}} \mathrm{vs}^{\mathrm{W}} \mathrm{WT}^{+20 /+200} \mathrm{p}<1 * 10^{-10}, \mathrm{H}^{-\mathrm{R}^{-}}$ ${ }^{R U}$ vs. $H / R^{+20} p=0.4238, H / R^{-R U}$ vs. $H / R^{+200} p<1 * 10^{-10}$ ). (C) Direct comparison of the mean lifespans in flies overexpressing the wild type and the mutant Narf allele. An induction with $200 \mu \mathrm{M}$ of RU (red) is affecting survival rate in a higher magnitude than induction with $20 \mu \mathrm{M}$ of RU (green). Overexpression of the mutant Narf H/R allele induced by $200 \mu \mathrm{M}$ of RU is more severe than the wild type allele under the same conditions (log-rank test: $\mathrm{WT}^{+200}$ vs. H/R $\mathrm{R}^{+200} \mathrm{p}<1 * 10^{-10}$ ) Significant differences can be also detected without induction (log-rank test: $\mathrm{WT}^{-\mathrm{RU}}$ vs. H/R ${ }^{-\mathrm{RU}} \mathrm{p}<1 * 10^{-10}$ ) and using $20 \mu \mathrm{M}$ of RU (log-rank test: $\mathrm{WT}^{+20} \mathrm{vs}$. H/R ${ }^{+20} \mathrm{p}=4.6$ $* 10^{-5}$ ). Statistical analyses were performed by OASIS, illustration was done using GraphPad Prism6.

\subsubsection{Knock-down of Narf did not significantly increase nuclear size in muscle cells}

Until now it is not clear if the dominant human patient mutation represents a gain-of-function or loss-of-function allele. In order to check for cellular effects in a knockdown situation a long hairpin structure specific for the fourth exon of Narf, available at VDRC, was muscle specifically expressed and a thorax staining against Dm0, Narf and TRITC phalloidin was performed after 15 days of RU induction. Muscle nuclei of noninduced control flies were quite similar in appearance compared to previous experiments (Figure 29), showing a reproducible robustness for this approach. The knockdown of Narf resulted in a high variance in nuclear size and led to the observation of enlarged muscle nuclei (Figure 33 B, C). However, a subsequent quantification revealed that there is no statistical relevance for the observed effects. In order to control the efficiency of the Narf knockdown an immunofluorescence analysis was performed. However, the Narf antibody staining is associated with unspecific background preventing a following quantification. But, using $200 \mu \mathrm{M}$ of RU seems to affect Narf protein localization in a higher extend than a weaker induction with $20 \mu \mathrm{M}$ of RU, suggesting a proper expression of the hairpin structure. 
A MHC-GS Gal4 Narf-RNAi - RU (Control)
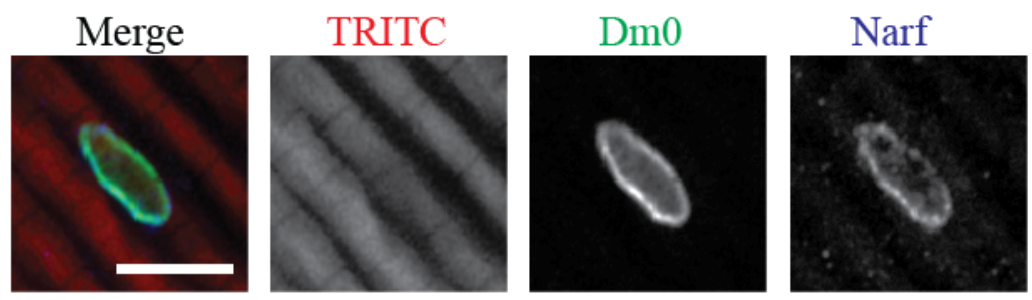

B MHC-GS Gal4 Narf-RNAi + $20 \mu \mathrm{M}$ RU
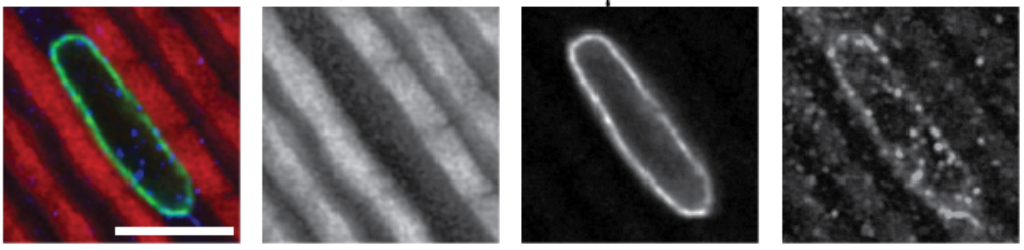

C MHC-GS Gal4 Narf-RNAi + $200 \mu \mathrm{M}$ RU
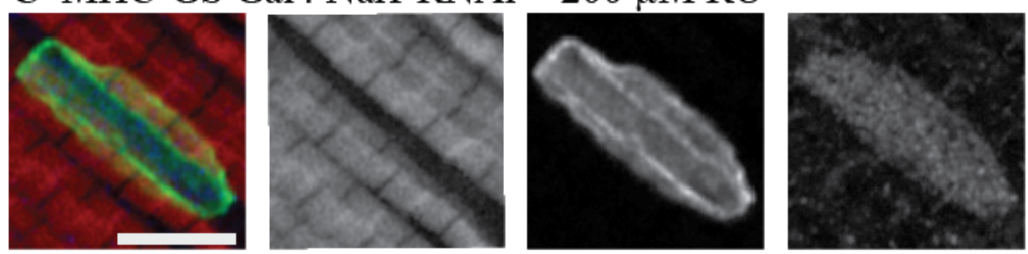

D

ns

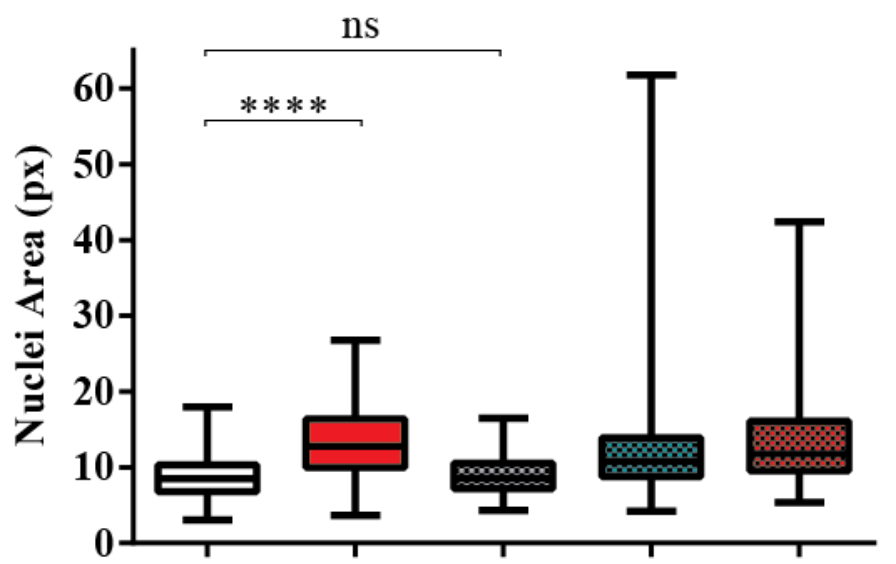

Unpaired $t$ test:

ns $\quad \mathrm{P}>0.05$

$* * * * \quad \mathrm{P}<0.0001$

Control $\left[\begin{array}{l}\square-\text { RU, MHC-GS Gal4; WT } \\ +\mathbf{2 0 0} \boldsymbol{\mu M} \mathbf{R U}\end{array}\right.$

$(\mathrm{N}=5 / 310$ nuclei)

$(\mathrm{N}=5 / 162$ nuclei)

$\operatorname{Narf-RNAi}\left[\begin{array}{ll}\text { - RU, MHC-GS Gal4; UAS-Narf RNAi } & (\mathrm{N}=3 / 89 \text { nuclei }) \\ +\mathbf{2 0} \boldsymbol{\mu M} \mathbf{R U} & (\mathrm{N}=4 / 67 \text { nuclei }) \\ +\mathbf{2 0 0} \boldsymbol{\mu M} \mathbf{R U} & (\mathrm{N}=4 / 82 \text { nuclei })\end{array}\right.$ 
Figure 33: Narf knock-down did not affect nuclear size

(A-C) Immunofluorescence analyses of thorax samples. Knock-down situation using $20 \mu \mathrm{M}$ (B) and $200 \mu \mathrm{M}$ of RU (C) after 15 days of induction. Non-induced flies were used as control (A). Immunostaining was performed with Narf, lamin Dm0 as an indicator for nuclear envelopes and TRITC phalloidin was used as a marker for muscle tissue. In both knock-down situations a higher variance in nuclear size was observed which was not seen in RU treated wild type flies. Representing pictures are shown in B and C. Scale bar: $5 \mu \mathrm{m}$. (D) Quantification of the nuclei area. Wild type flies crossed with the MHC enhancer and treated with $200 \mu \mathrm{M}$ of RU showed an increase in nuclear size verifying that RU alone has an effect on nuclear size compared to non-induced situations ( $\mathrm{Ctrl}^{-\mathrm{RU}}$ vs. $\mathrm{Ctrl}^{+\mathrm{RU}} \mathrm{p}=3.19 * 10^{-21}$, compare Figure 29). Narf knock-down in muscle cells induced by $20 \mu \mathrm{M}$ or $200 \mu \mathrm{M}$ of RU had no significant effect on nuclear size $\left(\mathrm{Ctrl}^{+200} \mathrm{vs}^{\mathrm{N}} \mathrm{Narf}_{\mathrm{RNAi}}{ }^{+20}: \mathrm{p}=\right.$ $0.2393, \mathrm{Ctrl}^{+200}$ vs. Narf RNAi $\left.{ }^{+200}: \mathrm{p}=0.0802\right)$. Quantification was done manually using ImageJ and statistical significance was calculated using unpaired $t$-test.

\subsubsection{Muscle specific knock-down of Narf during adulthood affected lifespan}

Since changes in nuclear size were detected after Narf RNAi expression in muscle cells, lifespan analyses were performed to verify if this phenotype might affect viability during adulthood. Indeed a muscle specific knock down of Narf significantly decreased survival rate of $\sim 24 \%$. Induced flies showed a 50\% mortality rate at day 44 compared to control flies which had a 50\% mortality at day 58. That this effect is independent of RU induction was shown before using a ten times higher RU concentration (Figure 31). Next, western blot analyses were performed to verify that the expression of a Narf specific hairpin structure mediates a decrease in Narf protein level (Figure 34 B). Thorax samples of induced and non-induced flies were tested at different time points during adulthood. However, a reduction was not observed as a Narf signal was only hardly detectable. An explanation can be given by a very weak thoracal expression which is consistent to tissue expression profile data available at http://flybase.org/reports/FBgn0262115. Furthermore, it is described that Narf is moderately expressed in imaginal discs. Due to this it was tested if a wing disc specific Narf knockdown controlled by the MS1096 driver is inducing morphology effects in adult wings (Supplement Figure 5). Down regulation of Narf in the wing blade caused wing phenotypes. However, a wing specific overexpression of Narf had no effects, suggesting the functionality of the hairpin mediated knock down. RNA sequencing analysis finally revealed an exon based down regulation of Narf and confirmed the previous data (Supplement Table: 5). 


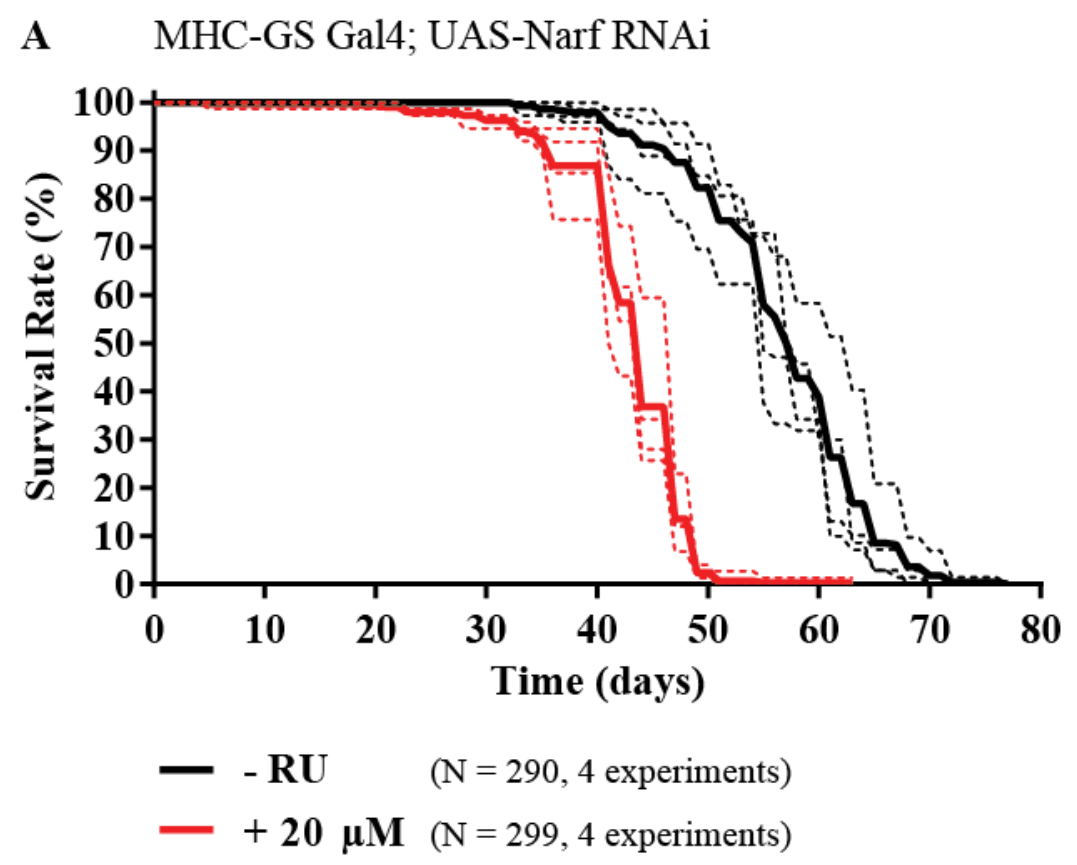

B

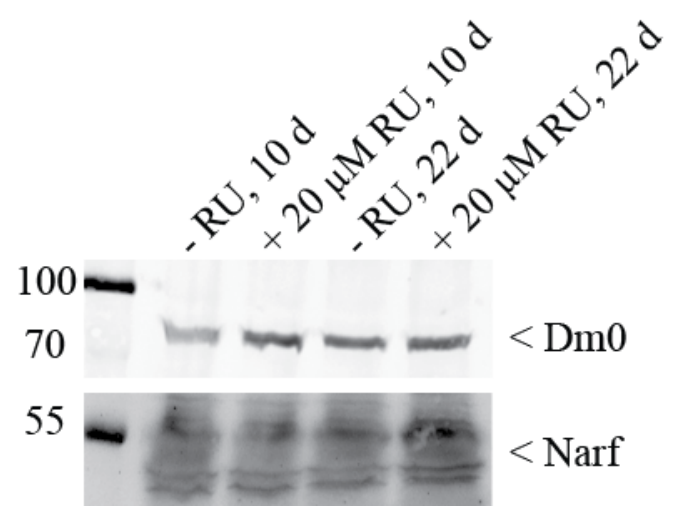

Figure 34: Muscle specific knockdown of Narf decreased lifespan

(A) Lifespan analyses of Narf-RNAi expressing flies treated with $20 \mu \mathrm{M}$ of RU (red) compared to non-induced control flies (black). Survival rate analyses were performed in four replicates (dashed lines) each with $~ 75$ males. The mean value was taken (solid lines) and used for statistical analyses. A muscle specific down regulation of Narf significantly decreased lifespan (log rank test: Narf $\mathrm{RNAi}^{-\mathrm{RU}} \mathrm{vs}$. Narf $\mathrm{RNAi}^{+20} \mathrm{p}<1 * 10^{-}$ ${ }^{10}$ ). (B) Western blot analyses of thorax samples at different time points after induction with $20 \mu \mathrm{M}$ of RU. Non-induced flies were used as control. The lysate of 2.5 thoraces was loaded and an antibody staining was performed against Narf and lamin Dm0 as loading control. A decrease in protein level after Narf RNAi expression was not observed since Narf is only weakly expressed within the thorax. 


\subsubsection{Narf is essential during adult stage}

It had been observed that a ubiquitous Narf knockdown controlled by $\alpha$-tubulin Gal4 resulted in a $100 \%$ lethality before flies reached adulthood. Western blot analyses of larvae extract revealed an efficient decrease in protein level (Supplement Figure 6). To study the effect of a ubiquitous Narf knockdown in adult flies and to circumvent the problem of lethality lifespan analyses were performed using the inducible actin GeneSwitch Gal4 driver line. Narf is essential during adulthood as induced flies showed a decrease in survival rate already in week one. An interesting observation was that a subpopulation of flies of approximately 5\% was able to survive and showed a longer lifespan compared to control flies. In order to investigate the reason western blot analyses were performed using fly extract at different time points after induction. An astonishing observation was that contrary to the assumption an increase in protein level was observed after 11 days and became more dominant over time. As $90 \%$ of the flies are still alive at this time point it seems that Narf was actively up regulated in the majority of induced flies, suggesting a specific function. An up-regulation of Narf might be associated with an organismal stress response as induced flies show a high lethality rate. 

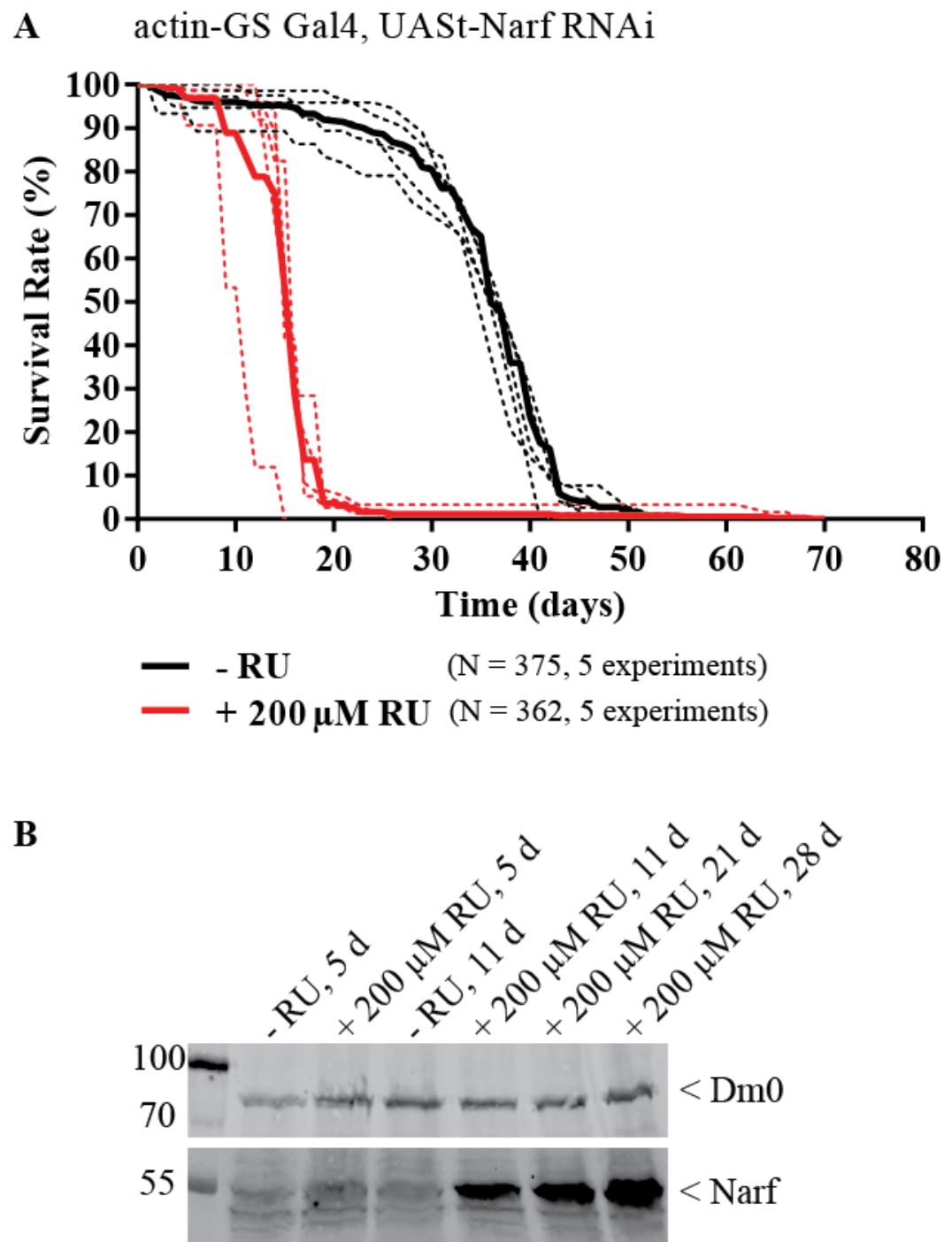

Figure 35: Ubiquitous down regulation of Narf during adulthood severely affected survival rate

(A) Lifespan analyses of Narf-RNAi expressing flies treated with $200 \mu \mathrm{M}$ of RU (red) compared to noninduced control flies (black). RNAi expression was controlled by actin GS Gal4 a ubiquitous driver line. Survival rate analyses were performed in five replicates (dashed lines) each with $\sim 75$ males. The mean value was taken (solid lines) and used for statistical analyses. A ubiquitous down regulation of Narf severely decreased lifespan (log rank test: Narf RNAi ${ }^{-R U}$ vs. Narf $\mathrm{RNAi}^{+200} \mathrm{p}<1 * 10^{-10}$ ). (B) Western blot analyses of fly samples at different time points after induction with $200 \mu \mathrm{M}$ of RU. Non-induced flies were used as control. The lysate of 2.5 males was loaded and an antibody staining was performed against Narf and lamin Dm0 as loading control. Contrary to the assumption an increase in protein level was observed after eleven days and became more dominant over time. 


\subsubsection{Gene expression is changed in Narf overexpressing or down regulating situations}

The change in Narf protein expression is affecting Drosophila on organismal level. To gain more insights about cellular changes upon muscle specific overexpression or down regulation of Narf and to get an idea of its role in biological mechanisms the transcriptom of coding genes from thorax samples was analyzed. RNA was extracted from ten thoraces in four biological replicates for each genotype (OE Narf WT, OE Narf H/R, Narf-RNAi). Narf down regulating flies, mediated by RNAi, were treated with $20 \mu \mathrm{M}$ of RU, whereas an overexpression was induced by $200 \mu \mathrm{M}$ of RU. Induction was performed for 10 days. Subsequent RNA sequencing analyses were performed at the Microarray and DeepSequencing Facility in Göttingen. It was shown before that RU induction itself had effects on nuclear size (Figure 29), suggesting that it might also influence gene expression level. Previous RNA sequencing experiments of the Großhans laboratory performed by R. Petrovsky confirmed that the gene expression profile is altered between thorax samples of induced and non-induced MHC-GS Gal4 flies. In order to enable the identification of Narf specific candidates independent of RU treatment, induced MHC-GS Gal4 thoraces were used as control. At that time the experiment was performed in triplets and induction was done with $200 \mu \mathrm{M}$ of RU for 5 days.

After analysis of the gene expression profiles, candidate lists for Narf overexpressing situations were aligned against the candidate list of the RU treated control situation. This led to an identification of 177 candidates for the overexpressed Narf wild type allele and 266 candidates for the mutant Narf H/R allele (Figure $36 \mathrm{~A}$ ). A comparison of these candidates showed that 139 can be found in both lists. The identification of the top 50 candidates, dependent on the 25 highest and lowest log2fold changes, revealed a high amount of similarity as 30 candidates were found in both lists (Supplement Table 3, 4). This indicates that the Narf H/R allele behaves almost like the wild type Narf. Genotype specific genes are referred as group “0, red”. The identification of Narf (CG17683) for being the highest up regulated candidate showed that induction as well as RNA sequencing analysis worked. An interesting observation is that especially genes involved in cellular detoxification (e.g. Cyp4p3, GstE14, Ugt36Bb), DNA/chromosome metabolism (e.g. phr, GATAe) and proteolysis (e.g. CG33159, CG18636) are detected in both Narf overexpressing situations. A down regulation of Narf led to the identification of 465 candidates, by which 97 were also found upon Narf(H/R) overexpression. Within the top 50 candidates 14 can be also found in 
the top 50 lists of the Narf overexpressing situations (Figure $36 \mathrm{~B}$ ). The remaining 36 candidates can be divided in to two subgroups. The first subgroup contains 16 candidates which can be found within the total lists for Narf overexpression. The second subgroup contains 20 candidates, which are specific for a down regulation of Narf (Supplement Table: 5, Group 0). The most interesting candidates are Cyp12d1-d and mthl8. Cyp12d1-d is also involved in cellular detoxification and mthl8 (methuselah-like 8) is annotated to be involved in aging in Drosophila due to its homology with mth (methuselah) which caused a lifespan extension upon mutation (Lin et al., 1998 ). The similarity of gene expression response by overexpression and depletion of Narf suggests that both situations represent a loss-offunction situation.

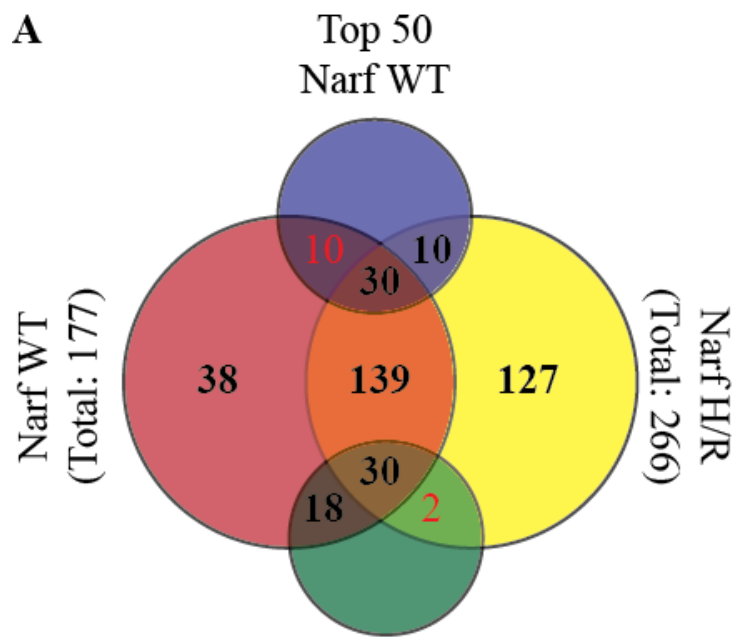

Top 50
B

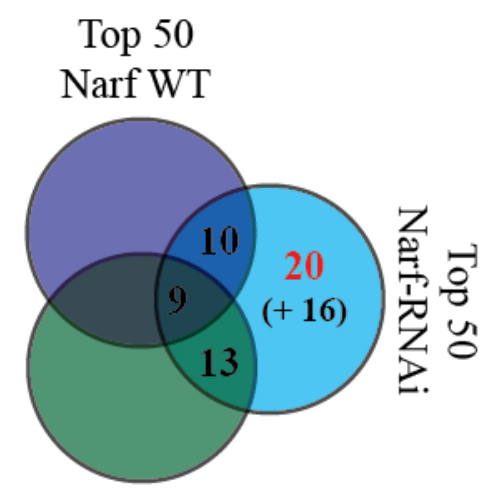

Top 50

Narf H/R

Figure 36: Gene expression is changed in altered Narf expressing situations

(A) Venn-diagram of gene expression changes comparing the overexpression of the wild type and the mutant Narf allele. The total lists of identified candidates are set in relation to the top 50 candidates. Red numbered candidates are gene type specific. (B) Venn-diagram of top 50 candidates for Narf down regulation in relation to Narf overexpression. Narf-RNAi specific genes are marked in red. Candidates which can be found within Narf OE total list but are not included within top 50 lists are set in clamps. 


\subsubsection{Klp68D is a Narf interaction partner}

In order to identify new protein interaction partner of Narf, a yeast- two hybrid assay was performed using the screening service from hybrigenics. The basic principle behind that assay is the restoration of an active transcription factor by direct interaction of two proteins fused with a DNA binding domain (DBD) or a Gal4 activation domain (AD). Protein interaction brings DBD and AD in a close proximity and allows transcriptional activation of a reporter gene. Reporter gene expression enables yeast cell growth on a selective medium and positive clones are later sequenced to identify new interaction partners (Brent et al., 1985, Fields et. al., 1989). In order to identify novel interaction partners a Lexa DNA binding domain was N-terminally fused to the full length Narf protein, based on the cDNA clone RE37350 (BDGP), and screened against a Drosophila embryo library. In total 116 million interactions were tested and 43 positive clones were identified. Final sequencing analyses lead to the identification of seven genes (BtbVII, CG5807, Klp68D, prd, Rpl22, RpS18 and troll). All steps were performed by the company. A high confidence in interaction was only given for Klp68D. All the other genes were categorized as technical artefacts or in case of Rpl22 described for being most likely an unspecific interaction. Klp68D belongs to the family of kinesin-like motor proteins, is primary expressed in the nervous system and described to be involved in the axonal transport of the choline acetyltransferase (Pesavento et al., 1994, Ray et al., 1999) 


\subsection{Is Narf involved in aging in C. elegans?}

Caenorhabditis elegans is an excellent and established model system in aging research. The $C$. elegans genome contains a single lamin gene (lmn-1). It is known that Celamin is critical for a normal lifecycle and is involved in aging as the reduction of the lamin levels during postembryonic development shortens lifespan. Furthermore, age-dependent changes in nuclear morphology such as an increased abnormal shape, extensive nuclear stretching and fragmentation have been described (Haithcock et. al., 2005). It was demonstrated that the treatment with farnesyl transferase inhibitors (FTI) can reverse these age dependent changes in nuclear morphology, showing a dependency on farnesylation. However, lifespan was not affected (Bar et. al., 2009, Bar and Gruenbaum 2010).

The Narf homologue in C. elegans is Oxy-4. Oxy-4 was identified in a screen for mutants with an increased sensitivity to oxidative stress. Importantly this study also reported that oxy-4 mutants had a shortened lifespan of about 20\% (Fujii et al., 2009).Interestingly, the identified mutant strain has a single point mutation at the homologues position of the human patient mutation. However, the molecular mechanism remains unclear and a connection to the nuclear lamina and lamin farnesylation was not made. The aim of this study was to analyze the functional relationship between Oxy-4 and Ce-lamin and to investigate how they contribute to aging.

\subsubsection{Oxygen sensitivity of oxy-4 mutants}

For my analyses, the oxy-4 (qa5001) worms strain was used, which was identified by Fuji et al., in 2009. I therefore rechecked the strain and tried to reproduce the previously described results. One of the hallmarks for this strain is an increased sensitivity to oxidative stress. To test this phenotype, an oxygen sensitivity assay was performed by culturing eggs in the presence of $75 \%$ of oxygen. My analysis confirmed the oxygen-sensitivity of the oxy4 mutant worms, as they show a delay in development compared to oxy-4 mutant worms grown at normoxic conditions (21\% of oxygen) (Figure 37 A). However, I did not observe the described arrest in L1-L2 stage with 90\% of oxygen (Fujii et. al., 2009). Furthermore, oxy-4 mutant worms were developmentally delayed even when grown at normal conditions compared to wild type (Figure $38 \mathrm{~A}$ ). Sequencing of the oxy-4 genomic locus confirmed the point mutation in the oxy-4 gene that led to a change from aspartic acid (D) to asparagine $(\mathrm{N})$ at codon 373 in the worm strain (Figure $37 \mathrm{~B}$ ). 


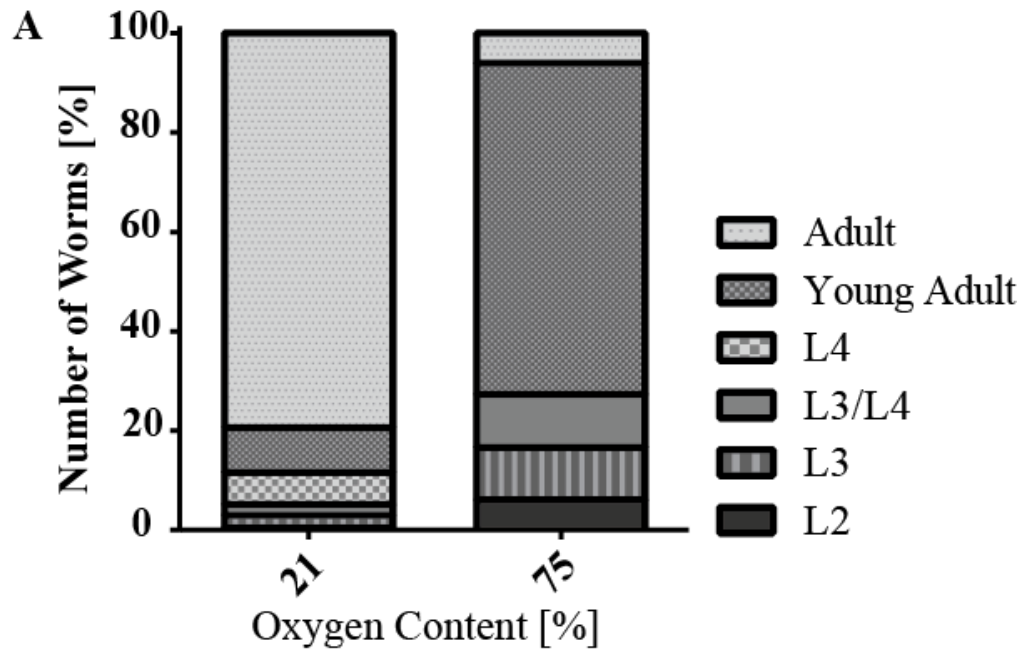

B

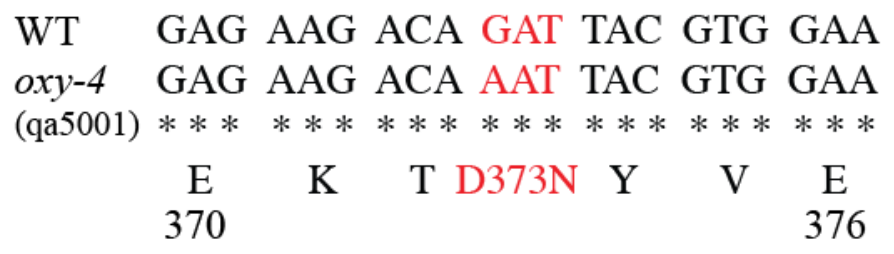

Figure 37: oxy-4 mutants were sensitive to oxidative stress

(A) Nematode embryos were incubated in the presence of $21 \%$ and $7 \%$ percent of oxygen and the developmental stages were determined after 5 days. Mutant worms incubated with 75\% of oxygen showed a delay in development. Statistical analyses were performed with $\mathrm{N}=233$ for $o x y-4$ mutant worms under normoxic and $\mathrm{N}=66$ under hyperoxic conditions. Developmental larval stages are referred as L2 to L4. (B) Sequencing results revealed that a point mutation leads to an amino acid exchange from aspartic acid (D) to asparagine $(\mathrm{N})$.

\subsection{2 oxy-4 mutants showed a developmental delay under normal conditions}

To further analyze the observed developmental delay under normal conditions, a time course analysis of oxy-4 mutant worms, wild type worms and oxy-4 RNAi worms was performed. For this purpose, worms were synchronized at L1 stage and then grown at $20{ }^{\circ} \mathrm{C}$ for three days (Figure $38 \mathrm{~A}$ ). The length of the worms was measured manually in ImageJ and data analyses was performed with GraphPad Prism6 (Figure 38 B). After 50 hours, wild type and oxy-4 RNAi worms reached adulthood and their final length, whereas oxy-4 mutant worms still grew after 71 hours. These results are consistent with the previous report (Fujii et. al., 2009), in which the authors described a delay in development of oxy-4 mutant worms 
of $1-1.5$ days compared to wild type at $20{ }^{\circ} \mathrm{C}$. The difference in phenotype between RNAi treated and mutant worms can be explained by an inefficient down-regulation of Oxy-4 via RNAi. Quantitative real-time PCR-analyses of oxy-4 RNAi treated wild-type worms revealed a remaining gene expression of $45 \%$ compared to wild-type worms treated with an empty vector control (EV-RNAi) (Figure $44 \mathrm{~B}$ ).

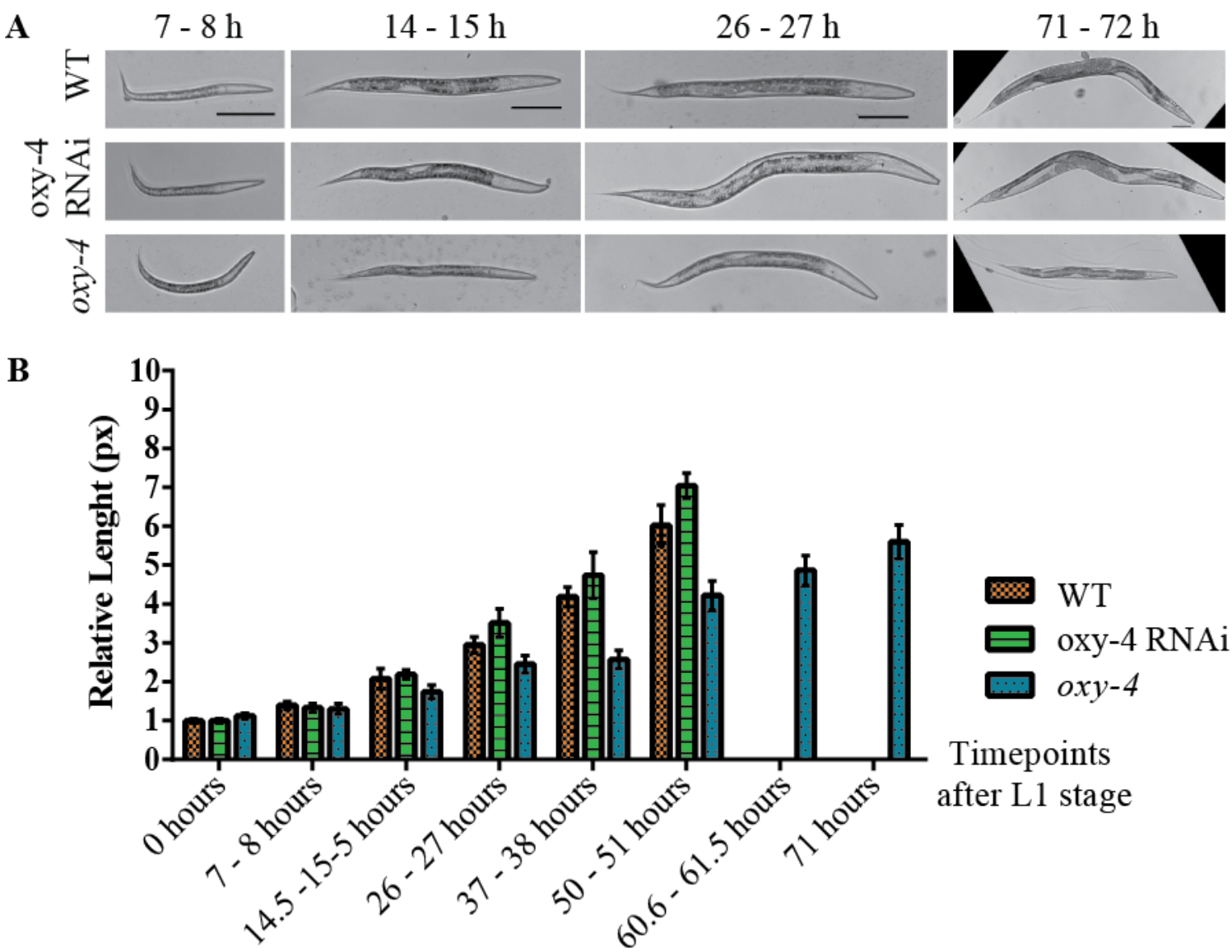

Figure 38: Developmental delay of oxy-4 mutants under normoxic conditions

Time course analysis of wild type, oxy-4 RNAi treated wild type and oxy-4 mutant worms. All genotypes were synchronized to L1 stage and body lengths were analyzed at distinct time-points. Representative pictures are shown in A. Quantification of worm lengths at different time points is presented in B. oxy-4 RNAi treated worms showed a similar growth as wild type worms, whereas the development of oxy-4 mutant worms is delayed.

\subsubsection{Unchanged muscle structure in oxy-4 mutants}

In $C$. elegans two main types of muscles can be found: the striated and the nonstriated muscles. Previous studies have demonstrated that a specific lamin mutation in $C$. 
elegans, which reflects the HGPS situation, leads to disorganization of sarcomeric actin filaments in striated body-wall muscle already at L4 stage (Bank et al., 2012). Furthermore, the human patient with the dominant de novo mutation in Narf showed an effect on muscle structure. I next examined whether oxy-4 mutant worms are also affected in their muscle structure. Worms at different time points during development were collected and the striated muscle structure of the body wall was analyzed by phalloidin staining. Compared to wild type worms, no morphological changes in muscle structure were visible in oxy-4 mutant worms even after 5 days of adulthood (Figure 39).

In addition, I checked the effect on the non-striated muscles in C. elegans. For this purpose, the pharynx was analyzed. As the food intake correlates with growth in C. elegans, and a developmental delay in oxy-4 mutant worms was observed, I wondered if a connection to a malfunctioning pharynx can be found. To check the muscle function a pumping experiment was performed, counting the grinding movement during pumping. Indeed, a statistical significant difference in pumping behavior between wild type and oxy-4 mutant worms was detected (Figure 40). Next I examined if this effect was due to morphological reasons. Therefore, the pharynx structure was analyzed by phalloidin staining. However, no aberrations in pharynx morphology were detected (Figure 41).

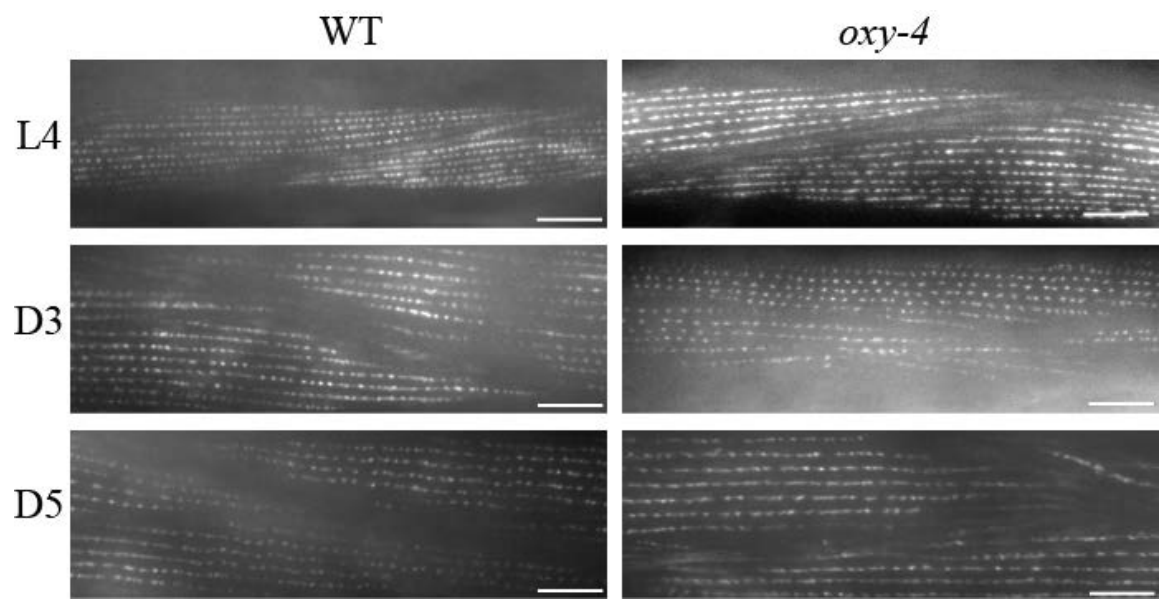

Figure 39: Striated muscle structure was normal in oxy-4 mutant worms

Wild type and oxy-4 mutant worms were stained against phalloidin at three distinct time points during development (L4-larval stage 4, D3- three days of adulthood, D5- five days of adulthood). Even after five days of adulthood no effects in body wall muscle structure were observed in oxy-4 mutant worms compared to wild type. Scale bar: $10 \mu \mathrm{m}$ 


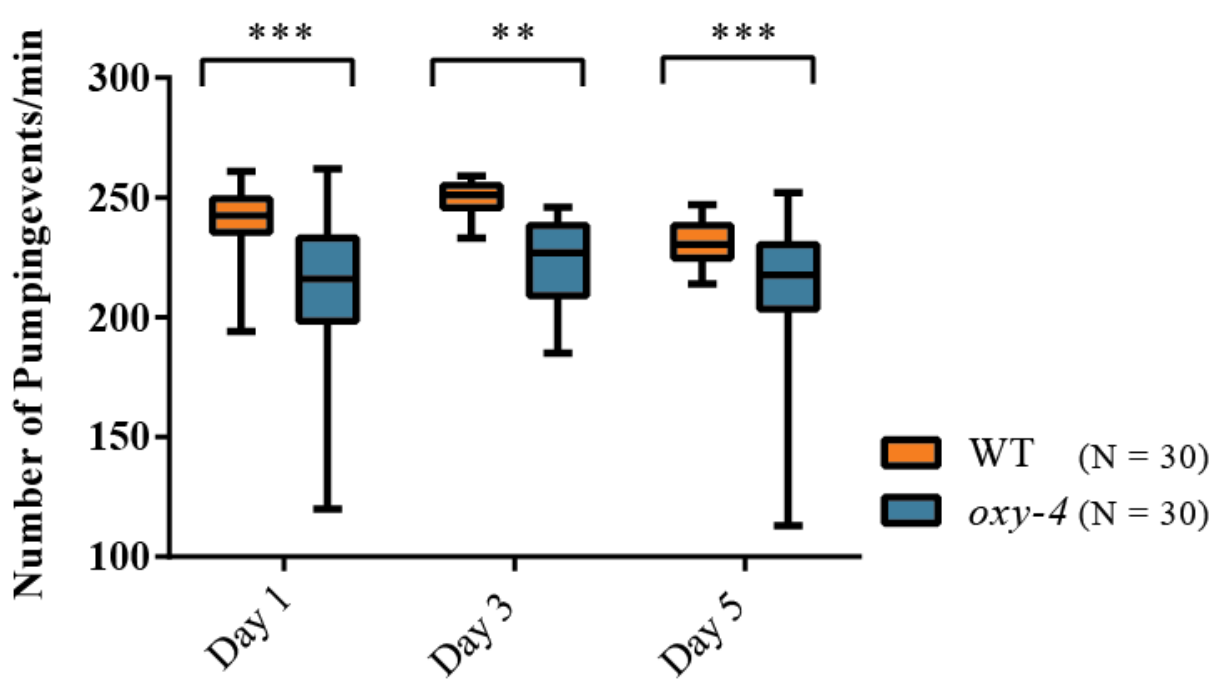

\section{Days of Adulthood}

Figure 40: oxy-4 mutants showed a decline in pharyngeal pumping

Statistical analyses of the pumping behavior of wild type and oxy-4 mutant worms at different time points after reaching adulthood. The error bars show min to max values. The difference in pharyngeal movement was statistically significant $\left(* * \mathrm{p} \leq 0,004 ;{ }^{* * *} \mathrm{p} \leq 0,001\right.$, unpaired $t$-test). Illustration was done by GraphPad Prism 6.
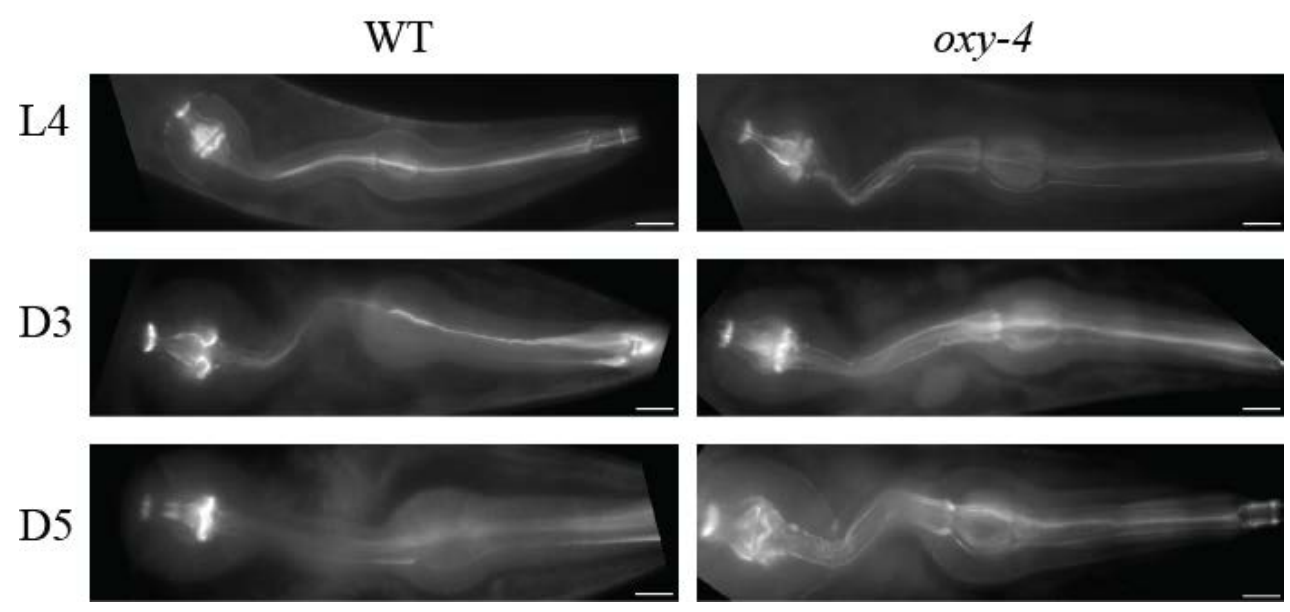

Figure 41: oxy-4 mutant worms were normal in non-striated muscle structure

Morphological pharynx analysis of wild type compared to oxy-4 mutants was carried out by phallodin staining at different time points during development (L4-larval stage 4, D3- day three of adulthood, D5- day five of adulthood). Even after five days of adulthood no effects in non-striated muscle structure can be observed in oxy-4 mutant worms. Scale bar: $10 \mu \mathrm{m}$ 


\subsubsection{Nuclei morphology and Ce-lamin farnesylation were not affected in oxy-4 mutants}

Ce-lamin and Oxy-4 are involved in aging, as a shorter lifespan was observed in their mutant situations (Fujii et. al., 2009, Haithcock et. al., 2005). A link to the nuclear lamina and the Ce-lamin farnesylation state was still unrevealed. To investigate the effect to the nuclear lamina, a Ce-lamin antibody staining of L4 larvae of the wild type strain and oxy-4 mutants was performed. I examined the appearance of muscle and hypodermis nuclei. In oxy-4 mutants a small number of nuclei showed an aberrant phenotype (Figure $42 \mathrm{~B}, \mathrm{E}$ ). However, this effect was also observed in the control situation, suggesting that a distinct amount of nuclei naturally showed this lobulations and infoldings. Statistical analyses confirmed that the observed effect on nuclei morphology is not significant (n.s.) (Figure 42 C, F). As it is supposed that the effect on the nuclear envelope disruption becomes more severe with age, it is possible that an effect can be detected in older worms. 
A
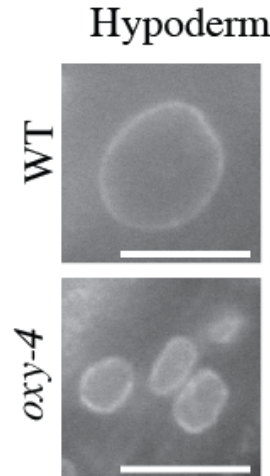

B

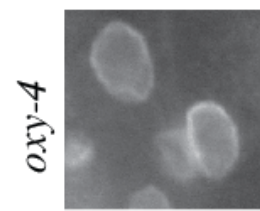

C

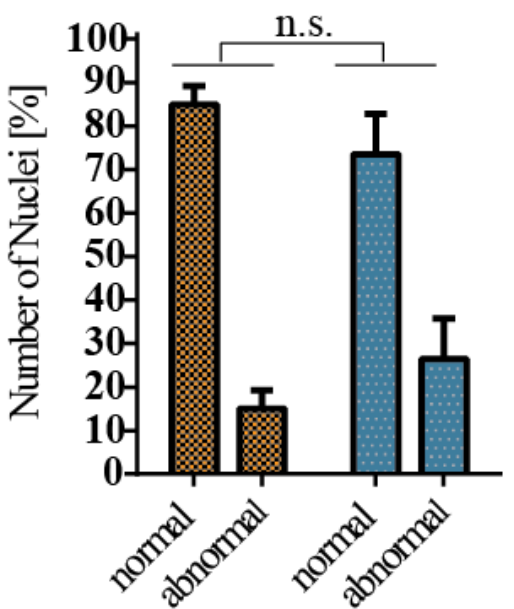

WT $(\mathrm{N}=4$, nuclei 95)

oxy-4 ( $\mathrm{N}=5$, nuclei 169)
D Muscle nuclei
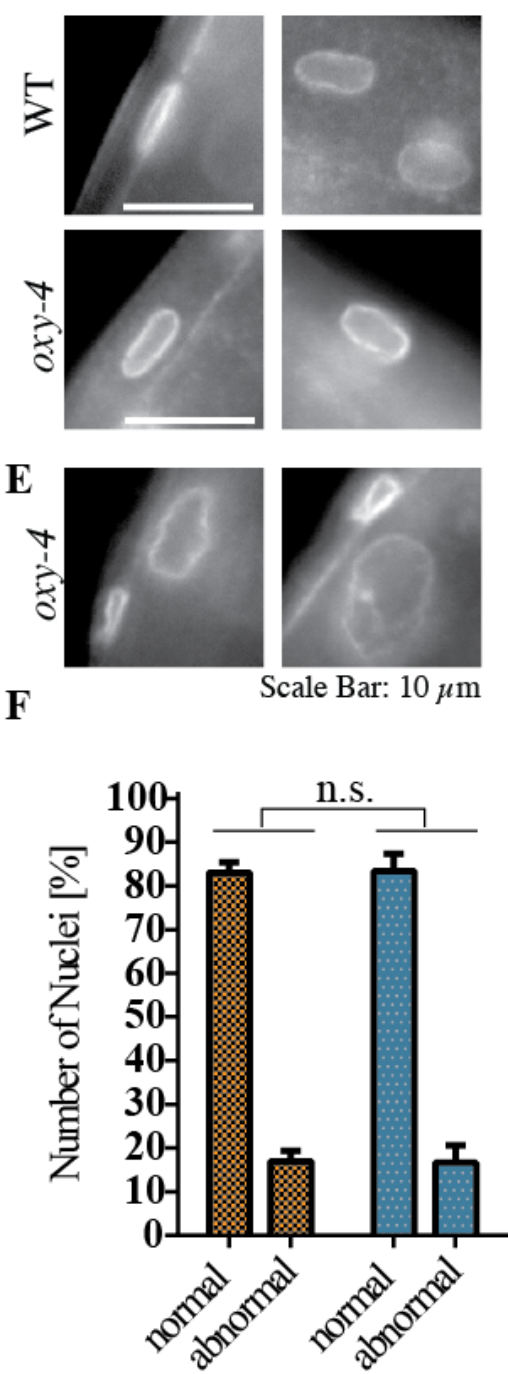

$(\mathrm{N}=4$, nuclei 53)

$(\mathrm{N}=5$, nuclei 130)

Figure 42: Nuclear morphology was not affected in oxy-4 mutant worms at larval stage

Nuclei morphology in wild-type and oxy-4 mutant worms was analyzed by Ce-lamin immunostaining. The nuclei appearance of two different tissues was studied: (A-C) hypodermis nuclei and (D-F) muscle nuclei. Nuclei which were referred as abnormally shaped are shown in B and E. Statistical analyses showed that these deformations occured in the same ratio also in wild-type worms and the number of misshaped nuclei is not significantly (n.s) higher in oxy-4 mutant worms. Statistical analyses were performed with at least four different worms of each genotype. Nuclei were counted, the appearance was studied and grouped to normal or abnormal shaped. As this approach is subjective, this analysis was performed thrice. The number of nuclei shown in C and $\mathrm{F}$ demonstrates the mean number of analyzed nuclei. 
To investigate the effect on the farnesylation state of Ce-lamin in oxy-4 mutants, a band shift assay was performed. Therefore, protein extracts of L4 larvae of wild type and oxy-4 mutant worms were separated via a SDS-PAGE and analyzed by Western-Blot experiment for a difference in Ce-lamin size (Figure 43). As positive control, L4 staged wild type worms were treated with fdps-1 RNAi until 5 days of adulthood to downregulate polyprenyl synthetase, which directly inhibits the production of farnesyl-PP (Bar and Gruenbaum in 2010). However, it was not possible to detect a band shift between wild type and the positive control (wild type + fdpsi RNAi). Thus, it remains an open question, if the farnesylation of Ce-lamin is affected in oxy-4 mutants. The fact that the Ce-lamin immunostaining of wild type worms and oxy-4 mutants looked quite similar (Figure $42 \mathrm{~A}$ ), suggests an unaffected farnesylation state of Ce-lamin.

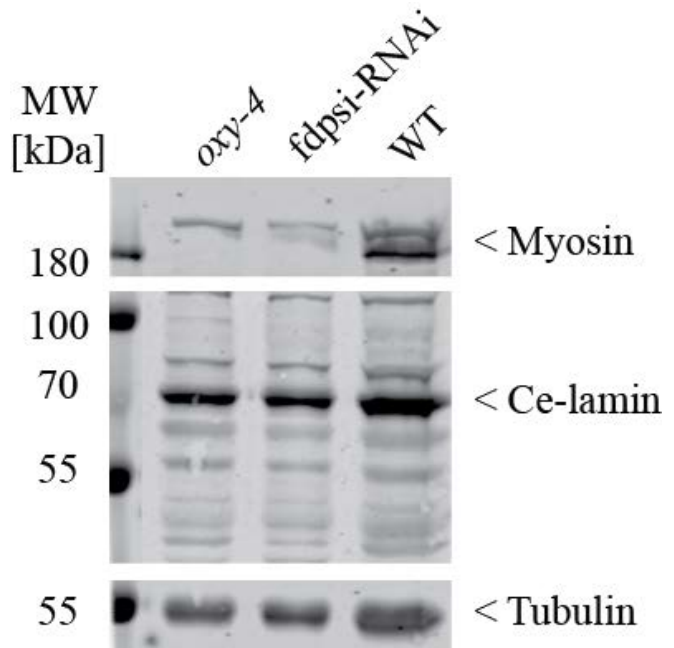

Figure 43: A lack of farnesylation in Ce-lamin did not result in a protein shift.

Western blot analysis of oxy-4 mutants, wild type worms treated with fdpsi-RNAi and non-treated control worms. Ce-lamin was detected at $~ 70 \mathrm{kDa}$. Downregulation of polyprenyl synthetase was used as positive control but did not lead to a change in Ce-lamin protein size. According to this, no effects on Ce-lamin in an oxy-4 mutant background were observed. Myosin and tubulin staining were used as loading controls.

\subsubsection{Do Oxy-4 and Ce-lamin act via the same pathway?}

The advantage of $C$. elegans in aging research is its short lifespan of three weeks. Thus, aging experiments can be conducted within weeks. In addition, the genetic background which is a big and cumbersome problem in Drosophila plays no role, as the animals are hermaphrodites. Due to this, I conducted lifespan assays to reveal the functional relationship between Ce-lamin and Oxy-4. Therefore, I first performed lifespan experiments by using oxy-4 RNAi. However, no difference to control worms was detected (Figure 44 A). To 
examine if this effect was due to an insufficient down-regulation of Oxy-4, a qPCR-analyses were performed (Figure $44 \mathrm{~B}$ ). The result confirmed that RNAi treatment was insufficient and a gene expression of $45 \%$ remained. Gene expression of Imn-1 instead was efficiently reduced, which was comparable to previous published data (Zuela et al., 2016).

A

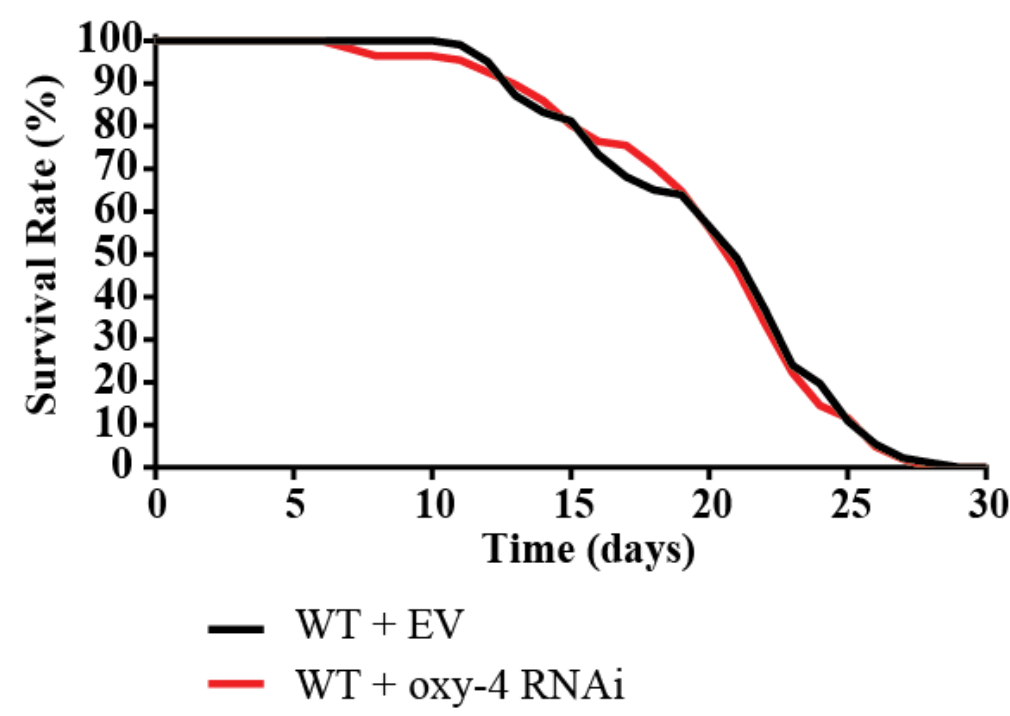

B

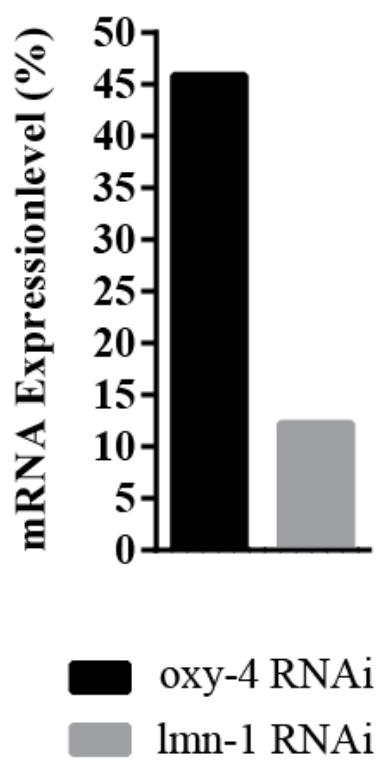

Figure 44: Inefficient knockdown of oxy-4

(A) Lifespan analyses of wild type worms treated with oxy-4 RNAi or an empty vector control RNAi L4440 (EV). For each condition 100 L4 larvae were separated with ongoing RNAi treatment and the survival rate was determined. The assay was performed at $20^{\circ} \mathrm{C}$. Statistical analysis was performed with OASIS, log-rank test $=$ 0.7169. (B) qPCR-analyses of wild type embryos grown on either oxy-4 or $l m n-1$ RNAi plates at $20^{\circ} \mathrm{C}$. L4 staged worms were used for mRNA extraction with subsequent cDNA preparation. Wild type worms treated with an empty vector (L4440) were used as control to determine the reduction in gene expression. Knockdown of Ce-lamin was used as positive control, as this RNAi construct was verified (Zuela et al., 2016). Lifespan and qPCR-analysis of oxy-4 RNAi revealed that the down-regulation of oxy-4 was not sufficient and had no effect on organismal aging.

Finally, I tested if Oxy-4 and Ce-lamin contribute to aging via the same pathway. Therefore, wild type and oxy-4 mutant worms were treated with either an empty vector (EV) or lmn-1 RNAi. As it is already described that down-regulation of Ce-lamin in wild type background leads to a decrease in lifespan (Figure 45 A) (Haithcock al., 2005).The same effect was observed for oxy-4 mutant worms, consistent to already published data (Fujii et al., 2009). To study a functional relationship between Oxy-4 and Ce-lamin, lifespan analysis of oxy-4 mutant worms with lmn-1 RNAi treatment were carried out. There are two potential 
mechanisms. 1) If Oxy-4 and Ce-lamin contribute independently to aging, an additive effect is expected. 2) If Oxy-4 acts via Ce-lamin, single mutants will behave similar as the double mutant. In Figure 45 B the results of the log-rank test analyses are depicted. Based on this, Oxy-4 and Ce-lamin would act independently, as lifespan curves of oxy-4 mutants with and without lmn-1 RNAi were significantly different $(p=0.0126)$. It needs to be mentioned at this point that the number of worms for oxy-4 mutant situations was limited and this experiment was, due to experimental reasons, performed only once. Summarizing, it remains an open question if Oxy-4 and Ce-lamin act within the same pathway. To make a conclusion it will be necessary to repeat this approach.

A

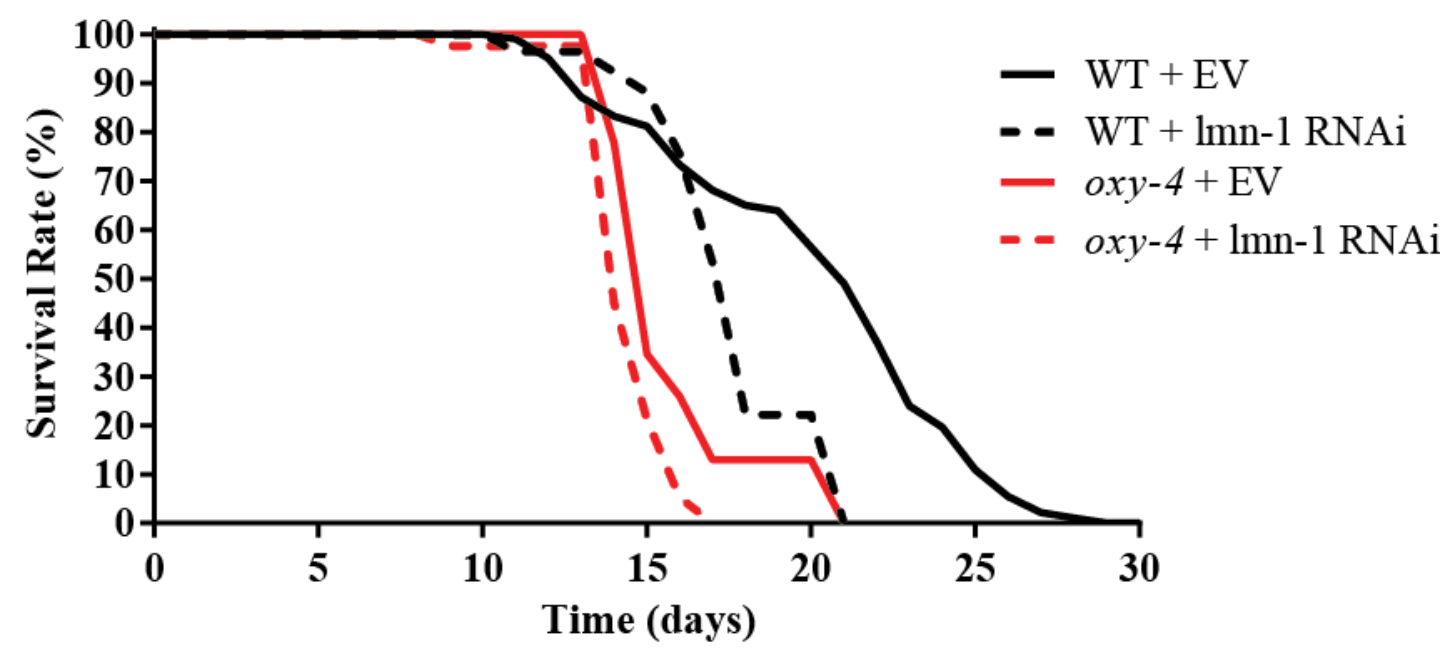

B

\begin{tabular}{cccc}
\hline vs & WT + lmn-1RNAi & oxy-4 + EV RNAi & oxy-4 + lmn-1RNAi \\
\hline WT + EV RNAi & $\mathrm{p}=0.0064$ & $\mathrm{p}=0.0082$ & $\mathrm{p}=1 * 10^{-10}$ \\
WT + lmn-1 RNAi & & $\mathrm{p}=0.0101$ & $\mathrm{p}=1 * 10^{-10}$ \\
oxy-4 + EV RNAi & & $\mathrm{p}=0.0126$ \\
\hline
\end{tabular}

Figure 45: Lifespan analyses of oxy-4 mutants with additional lmn-1 RNAi treatment

(A) Lifespan analyses of wild type and oxy-4 mutant worms. Embryos were grown on either empty vector RNAi L4440 (EV) plates or treated with Imn-1 RNAi. 100 L4 larvae for each wild type condition and 50 L4 larvae for oxy-4 mutant situations were separated with ongoing RNAi treatment and the survival rate was determined. Lifespan analyses were performed at $20^{\circ} \mathrm{C}$. (B) Statistical analyses were performed by OASIS (log-rank test). Illustration was accomplished by GraphPad Prism6. 


\section{Discussion}

\subsection{Kugelkern and its role in aging}

\subsubsection{The $k u k$ deficient fly line $\Delta 15$ might still contain recessive mutation(s)}

Kugelkern is connected to accelerated aging-related phenotypes in Drosophila as an overexpression caused nuclear morphological changes, a reduction in heterochromatin and shortened the mean lifespan of adult flies (Brandt et al., 2008). In order to investigate a loss-of-function situation in a more detail an established fly strain, homozygous for a kugelkern deletion ( $\Delta 15)$, was used in this study and further analyzed for its gene expression profile. RNA sequencing analyses revealed alterations in gene expression especially for genes involved in stress response (Figure 5, Supplement Table: 1). In order to analyze if this might be connected to accelerated aging phenotypes in kuk depleted flies, lifespan analyses were performed. $\Delta 15$ and wild type control flies had the same $50 \%$ mortality rate and showed only on a low significant level differences in lifespan (Figure 6 A). Since no connection between alterations in gene expression and mean lifespan was found the question arose if lifespan analyses have been performed with representative males even if the fly strain was easy to keep, which is indicative for a good viability and fertility. Furthermore, it has to be mentioned that the chromosome harboring the $\Delta 15$ deletion was cleaned as secondary lethality causing mutations had to be removed in order to establish a homozygous $\Delta 15$ fly strain (M. Clever). However, it might be that recessive mutations retained after chromosomal cleaning. In order to analyze this aspect survival rate analyses during development were performed. Indeed, homozygous $\Delta 15$ animals showed a decrease of approximately $70 \%$ in embryonic survival compared to wild type animals. Survival rate analyses of animals which were only homozygous for a kuk depletion ( $\Delta 15 /$ Def) showed a wild type like viability, suggesting that the observed lethality is independent of kuk (Figure 6 B). As RNA sequencing analyses were done with 6-8 hours staged embryos it might be reasonable that the embryos, which are about to die, are causative for the observed alterations in gene expression. This would be consistent to the identified candidate genes which are especially involved in stress response. Thus, it remains unclear if $k u k$ depletion by itself affects gene expression. 


\subsubsection{Proteolytic cleavage might be mediated by PEST-sequences}

Besides the lamins, Kugelkern is the only known nuclear protein which also contains a CaaX-motif for farnesylation. The hypothesis is that the morphological phenotypes in the wing and the eye observed upon tissue specific overexpression are mediated by farnesylation dependent protein complexes. In order to identify these farnesylation dependent interaction partners of Kugelkern I planned to perform Co-immunoprecipitation experiments using a modified GFP-nanobody followed by mass spectrometric analyses. Accordingly, genomic and GFP-tagged alleles of the wild type Kuk and a non-farnesylable mutant (KukC567S) were established and expressed in a kuk depleted background. However, subsequent rescue experiments showed that the GFP-tagged wild type Kuk was not able to rescue the nuclear elongation phenotype in kuk depleted embryos (Figure 7). Following analyses confirmed that the expressed GFP-Kuk is only partially functional as proteolytic cleavage is interrupting protein function (Figure $8 \mathrm{~A}$ ). As similar proteolytic cleavage products were detected in a UAS/Gal4 expressed situation which is rescuing the nuclear elongation phenotype, it is reasonable that this might be a dose dependent effect (Figure 9). Interestingly, proteolytic cleavage occurred especially at the C-terminal half of Kuk whereas GFP-fusion was directed to the N-terminal site (Figure 8 B). All GFP-Kuk transgenes were affected and Kuk-fragments already occurred within the total extract directly after embryo lysis, which suggest that proteolytic cleavage was a general problem and independent of the transgenes, the used Co-IP conditions or the genetic background. Furthermore, it was shown in immunofluorescence analyses that GFP-Kuk partially miss-localized to the cytoplasm, suggesting a loss or disturbance of required localization elements like the nuclear localization signal (NLS).

Furthermore, it was observed that the proteolytic cleavage of GFP-Kuk seemed to affect preferentially two sites as two major cleavage products were detected with a molecular mass of approximately 70 and $100 \mathrm{kDa}$ (Figure $8 \mathrm{~A}$ ). In silico analyses revealed that Kugelkern contains two PEST motifs between the amino acid positions 268 - 299 and 487 - 524 (Supplement Figure 8), which were identified by the software epestfind (emboss.bioinformatics.nl/cgi-bin/emboss/epestfind). PEST motifs are proline (P), glutamic acid (E), serine (S) and threonine (T) rich amino acid sequences and have been found in the majority of fast degrading proteins (Rogers et al., 1986). Sequence analyses of nuclear proteins revealed this relationship that proteins with a structural function such as Histones and lamins which are supposed to be stable, do not contain PEST-sequences; whereas 
nuclear proteins with regulatory functions at least have one motif (Chevaillier et al., 1993). Consistent to this, no potential PEST motif was found for lamin Dm0 in Drosophila (Supplement Figure 9). However, it remains unclear why the N-terminal GFP-fusion of Kuk led to an activation of the cleavage signal. PEST-signals are described for being conditional and induced by different conditions such as light or phosphorylation (Rechsteiner and Rogers, 1996). So it might be also reasonable that the GFP-fusion led to protein fold changes which either made the PEST-motifs directly accessible or activated them indirect by exposing regulatory amino acids. However, western blot analysis of solubilized endogenous Kuk confirmed that these cleavage products also appear within the native situations as two additional protein bands with a molecular weight of approximately 55 and $75 \mathrm{kDa}$ were detected (Supplement Figure 2). Nevertheless, the majority of the Kuk protein stayed stable even after four hours of solubilization. By this, the requirement for an alternative Co-IP approach by a Kugelkern specific antibody coupled to protein A dynabeads is given. Farnesylation dependent interaction partners will be revealed by a comparison of wild type and non-farnesylable Kuk lysates. Transgenic KukC567S flies were successfully generated in this study and need to be crossed in a cleaned kuk deficient situation. However, the disadvantage of this approach is a missing elution step of Kuk-binding proteins from the beads, which will lead to a higher background. This problem will be minimized by a control experiment with the lysate of kuk depleted embryos.

\subsection{Narf is involved in aging in Drosophila}

The group of Prof. Wollnik (Institute for Human Genetics, Göttingen) recently identified a dominant de novo mutation in Narf in a human patient which shared clinical features to HGPS patients like reduced subcutaneous fat, mild osteoporosis and nuclear morphology defects (personal communication). Phenotypic similarities of the human patients, its initial identification by binding to farnesylated Prelamin A and the exclusive nuclear rim localization in HeLa cells (Barton and Worman, 1999) led to the hypothesis that Narf might represent a new progeria gene related to laminA. How Narf might contribute to aging or if the characterized human mutation represents a gain of function or a loss of function allele are not known yet. A first step to understand the underlying molecular mechanisms was to establish Drosophila as model system and to study the effect of Narf. In this study it was shown that the Drosophila homologue CG17683 indeed is involved in regulating aging processes. Even if this study just provides a starting point of Narf and its 
role in aging one criteria of being a member of "aging genes" was already fulfilled by showing that a loss-of function leads to a decrease in lifespan (Figure 34) whereas a gain-of function is prolonging Drosophila lifespan (Figure 24, Figure 25).

\subsubsection{Narf might have an essential role in Fe-S protein maturation}

Narf is known to possess an essential function for development in Drosophila, since four different lethal alleles (NC37, NC38, NC70 and NC109) are already identified (Coulthard et al., 2010). However, the time point of lethality was not further studied yet. Survival rate analyses of the two characterized lethal alleles (NC38 and NC109) showed a lethality during larval stage (Figure 20). Reasoning to this the lethal allele NC38 was proven to contain a premature stop codon (Figure 19) which might lead the nonsense-mediated mRNA decay, showing that a ubiquitous loss-of function results in lethality. Western blot analyses showed that Narf is probably maternally provided and a zygotic expression starts at the switch from embryonic to larval stage (Figure 21) which goes along with the observed larval lethality. Consistently, a tubulin driven down regulation by Narf-RNAi caused a preadult lethality phenotype of 100 percent penetrance.

In order to analyze a Narf loss of function effect during adulthood, the GeneSwitch system was used and ubiquitous down regulation of Narf was driven by actin upon induction with mifepristone (RU468). Thereby it was observed that the majority of the flies died within approximately three weeks, suggesting an essential function of Narf also during adulthood (Figure 35).

An explanation for the observed lethality might be given by an essential role of the Drosophila homologue in Fe-S protein biogenesis, as it was described for the yeast homologue Nar1 as well as for the mammalian homologue IOP1 (Narf-like) (Balk et al., 2004, Song and Lee, 2008). The maturation of Fe-S proteins is a quite conserved mechanism and can be simplified in four steps in yeast. Initially, a Fe-S cluster is assembled on a mitochondrial scaffold enzyme. Subsequently the cluster is released and transferred to a targeted apo-protein where it is finally integrated. Mitochondrial Fe-S proteins are solely dependent on the irron-sulphur-cluster (ISC) assembly machinery, whereas cytosolic and nuclear Fe-S proteins also requires the cytosolic iron-sulphur protein assembly (CIA) machinery (reviewed in Lill, 2009). Nar1 was described for being one of the CIA proteins and for this reason, Nar1 is essentially required for maturation of cytosolic and nuclear Fe-S proteins. Nar1 depleted yeast cells are lethal, which was explained by the need of Nar1 for 
the biogenesis of the cytosolic Fe-S protein Rlip. Depletion of Rlip led to a nuclear export defect of both ribosomal subunits and finally caused translational arrest (Balk et al., 2004, Don et al., 2004, Kispal et al., 2005, Yarunin et al., 2005).

That also the mammalian ortholog IOP1 is involved in maturation of cytosolic Fe-S proteins was shown in HeLa cells and MEFs (mouse embryonic fibroblasts) as knockdown of IOP1 is affecting the activity of different cytosolic Fe-S proteins but has no effect of tested mitochondrial Fe-S proteins or non Fe-S proteins (Song et al., 2009; Song and Lee, 2008, 2011). The described effects were shown to be specific for IOP1 as a knockdown for IOP2/Narf did not affect Fe-S protein maturation (Song and Lee, 2008). An IOP1 depletion in mice was shown to be lethal during early embryogenesis as well as in adults if the knockout was performed in an inducible way (Song and Lee, 2011). A functional explanation was given by the observation that an IOP1 knockdown in HeLa cells led to a global decrease in protein synthesis which finally caused growth impairment and a decreased cell viability as it was similarly seen in yeast before (Song and Lee, 2008).

Nothing is known about the Drosophila homologue and its putative role in Fe-S protein maturation yet. However, the mechanism is quite conserved and it might explain the observed lethality effects during development and adulthood as it was seen in yeast and mice before.

\subsubsection{Lifespan analyses as a tool to study aging}

Lifespan analyses are a central physiological test to study the effect of a distinct gene referring to aging. However, one of the most challenging task using lifespan analyses is to provide suitable controls. It is known that Drosophila lifespan can vary a lot dependent on genetic as well as on environmental conditions such as diet, infection, gender, mating status, temperature, humidity and circadian rhythm (reviewed in He and Jasper, 2014). To provide comparable situations all lifespan analyses were performed using synchronized males which were kept at $25{ }^{\circ} \mathrm{C}$ with 70 percent humidity and a day night shift of 12 hours. Furthermore a sample size of $\sim 200$-300 flies was used, the fly density was limited to $\sim 70$-80 flies per cage and lifespan analyses were performed in three to four replicates. A critical problem of a different genetic background was partially circumvented by using the GeneSwitch system. There the offspring of the same crossing are analyzed and gene expression is controlled by addition of the activator mifepristone (RU486) in a dose-dependent manner (Figure 27). The concentration of RU suggested for lifespan analyses is quite broad with a range from 
25 - $500 \mu \mathrm{M}$ (e.g. Copeland et al., 2009, Bahadorani et al., 2010, Biteau et al., 2010). However, the RU effect itself has to be considered carefully as it is supposed to influence Drosophila's food uptake probably to its bad palatability. Thus, changes in lifespan could be due to a dietary restriction effect. However, this kind of effect was observed for lownutrient food in most instances (Yamada et al., 2017).

In this study, high nutrition food was used and gene expression was induced with a maximal concentration of $200 \mu \mathrm{M}$ of RU. In order to validate if RU might affect life time under these conditions, lifespan analyses of wild type flies in the absence and the presence of $200 \mu \mathrm{M}$ of RU were analyzed. It was shown that RU had no effects on Drosophila lifespan (Figure 31), allowing the usage of the GeneSwitch system for survival analyses.

\subsubsection{Narf overexpression might increase intracellular stress defense mechanisms}

A lot of genes are described for shortening lifespan in Drosophila. However, talking about "aging genes" implicate the consideration of genes which have a beneficial effect on organismal aging. I showed in this study that Narf could be a new member oft these genes as as a loss-of function led to a decrease in lifespan (Figure 34) whereas a gain-of function prolonged Drosophila`s lifespan (Figure 24, Figure 25). RNAi experiments were performed in a muscle specific manner as it was shown that a ubiquitious downregulation caused lethality. Overexpression was achieved by an increase of the genomic copy numbers of Narf or by the tubulin-Gal4 controlled expression of a UAS-Narf transgene (Figure 24, Figure 25). Both gain-of functions situations were characterized by an overexpression starting already during embryogenesis. Western blot analyses confirmed an elevation in Narf protein level under the control of tubulin-Gal4 in larval stage (Supplement Figure 6) as well as during adulthood (Supplement Figure 4). How Narf might improve organismal aging on the molecular level still needs to be studied. However, a hint that an up-regulation of Narf might improve the stress response in aged flies and by this contribute to a delay in the aging process was given by Corbin et al., which identified the human IOP1 (Narf-like) as a key factor for hyperoxia protection in HeLa cells. They showed that the mRNA as well as the protein levels are increased for IOP1 in hyperoxia resistant cells and that the achieved overexpression was required for protection of cytosolic and nuclear Fe-S proteins in situations of hyperoxiainduced oxidative stress (Corbin et al., 2015). Fe-S proteins contain iron which makes them fragile especially in the presence of of oxidative stress as they are easily oxidized. An 
efficient protection of Fe-S proteins under these conditions is necessary as they are involved in a variety of different processes and essential for life (Johnson et al., 2005).

\section{A Gain-of function situation}
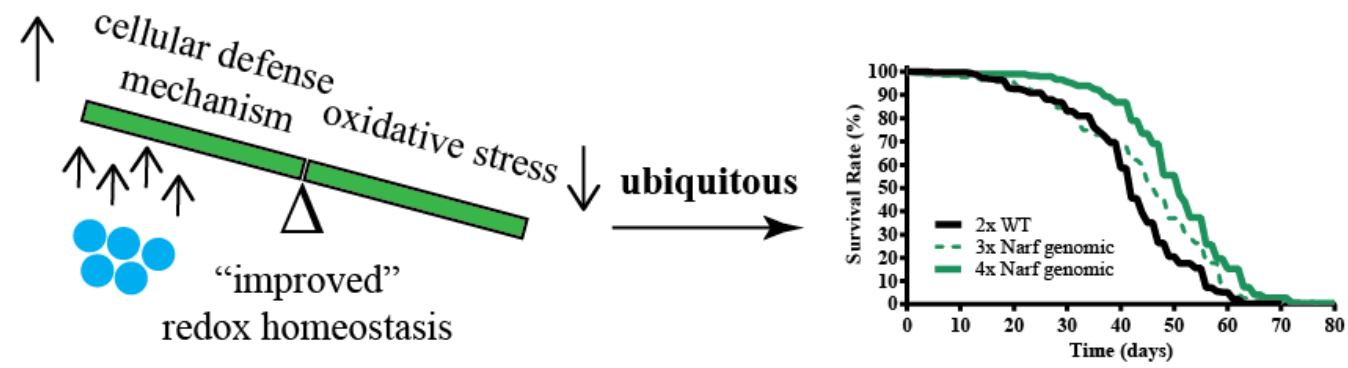

\section{B Loss-of function situation}

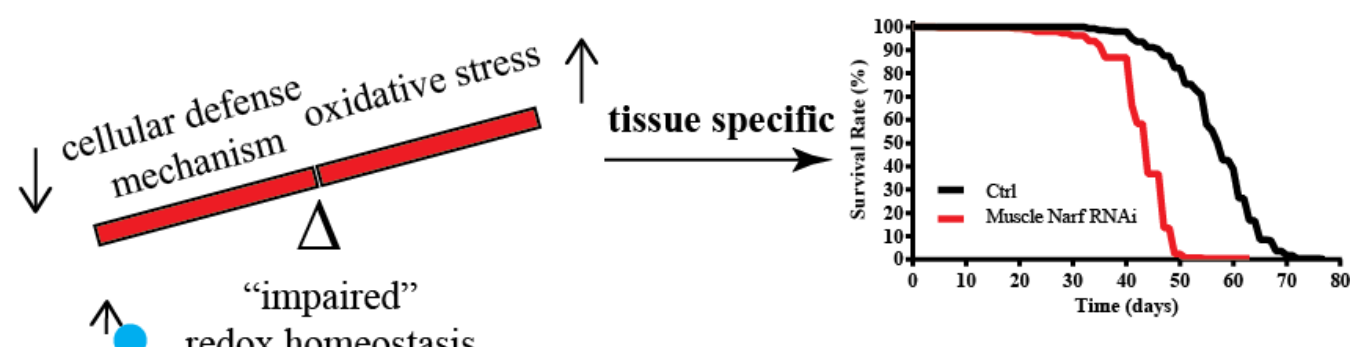

\section{Narf protein}

Figure 46: Model for the role of Narf and its contribution to aging in Drosophila

It was shown in this study that a ubiquitous overexpression of Narf led to a prolongation in lifespan, whereas a tissuespecifc down regulation caused a shortening in lifespan. I am hypothesizing that Narf is involved in balancing the cellular redox homeostasis which is mediated by its gene expression levels. High Narf protein levels might activate cellular defense mechanisms or might improve the protection of essential Fe-S proteins in the presence of oxidative stress as it was described for the human IOP1 (Narf-like) (Corbin et al., 20015). As Narf overexpression is beneficial for organismal aging, it is conceivable that Narf leads to an amelioration of oxidative stress occurring during normal aging. A loss of function instead might decrease the ability of cellular detoxification mechanisms or might cause an enhanced fragility of Fe-S proteins against oxidative stress which goes along with a decrease in lifespan. However, the moleculare mechanisms are not known yet and still needs to be studied.

An unexpected observation was made by the ubiquitinous downregulation of Narf during adulthood. As discussed before, the majority of the flies died within three weeks upon Narf downregulation, probably due to an essential role in Fe-S protein maturation. However, a subpopulation of approximately 5\% showed even a prolongation in lifespan, which was correlating with an increased Narf protein expression (Figure 35). Even if lifespan extension was not a general effect as the majority of the flies died, the effect of a Narf overexpression 
was indeed a general phenomenon. Western blot analyses showed an increase in Narf protein level starting at the second week correlating to a time point where approximately $75 \%$ of the RNAi expressing flies are still alive. Sample preparation was furthermore performed with the extract of ten flies, supporting the idea of a common effect.

However, the simplest explanation would be that an efficient down regulation of Narf was not induced. Knock down studies were performed using a long hairpin structure specific for the fourth exon of Narf. To analyze the general function of the Narf-RNAi construct different driver lines were used and Narf protein level were validated. In order to do this also thorax samples were checked for Narf protein expression after a muscle specific Narf knockdown (Figure 34 B). A decrease in protein level after Narf RNAi expression was not observed by western blot analyses. Time points of $10 \mathrm{~d}, 22 \mathrm{~d}$ and $30 \mathrm{~d}$ after induction were tested. However, a Narf signal was not detectable neither in control nor in RNAi samples, showing that Narf is only weakly expressed within the thorax. These data are consistent to tissue expression profile data (http://flybase.org/reports/FBgn0262115\#expression). To further test if Narf protein level is decreased immunofluorescence analyses of muscle nuclei were performed. However, after induction with $20 \mu \mathrm{M}$ still a staining of the nuclear rim was observed. Using $200 \mu \mathrm{M}$ of RU led to a more nucleoplasmatic staining of Narf (Figure 33 $B, C)$. But, it is not clear if the observed effects were specific or had to be considered as unspecific as the newly generated Narf antibody showed some background signal within stained thorax samples. Still, that the Narf antibody in general work good was shown in different tested conditions e.g. in immunostainings of S2 cells and embryonic amnioserosa cells as well as in western blot analyses (Figure 18). Thereby also larval extract of tubulin driven RNAi expressing animals was tested, showing an efficient down regulation of Narf protein level (Supplement Figure 6). This experiment showed first that the RNAi construct worked and second gave another proof for the establishment of a specific Narf antibody. Furthermore, it was observed that a wing specific Narf knock down induced morphological defects in adult wings (Supplement Figure 5), indicating a loss of function phenotype. RNA sequencing analyses of thorax samples finally revealed an efficient and exon based down regulation of the Narf transcript (log $\log _{2}$ ld: -3.10) upon muscle specific Narf RNAi expression. As the observed increase in Narf protein level did not originate from an inefficient mediated knock down, the question arose if this effect might be considered as Narf specific. 
Due to this it is conceivable that a ubiquitous knock down of Narf during adulthood (Figure 35) induced a cellular stress situation eventually based on a disturbed Fe-S protein maturation. The hypothesis is that this situation led to an elevation of Narf protein level in order to protect the cellular redox homeostasis. Furthermore, it was shown that the yeast homologue Nar1 is required for its own biosynthesis (Balk et al., 2004), suggesting a regulation via a negative feedback mechanism after knocking down. However, if the up regulation of Narf protein level is caused by an increase in gene expression or controlled by increase in protein stability might be shown in the future.

On the other hand, the majority of the flies died even after Narf overexpression, suggesting that the activation of the defense mechanism was too slow or the developed damage already too severe. But, the survival of a distinct subpopulation indicates the capability of this mechanism in stress protection. The fact that these flies even show a prolongation in lifespan compared to control flies indicates the induction of long lasting effects which might improve the cellular defense mechanism against elevated ROS levels.

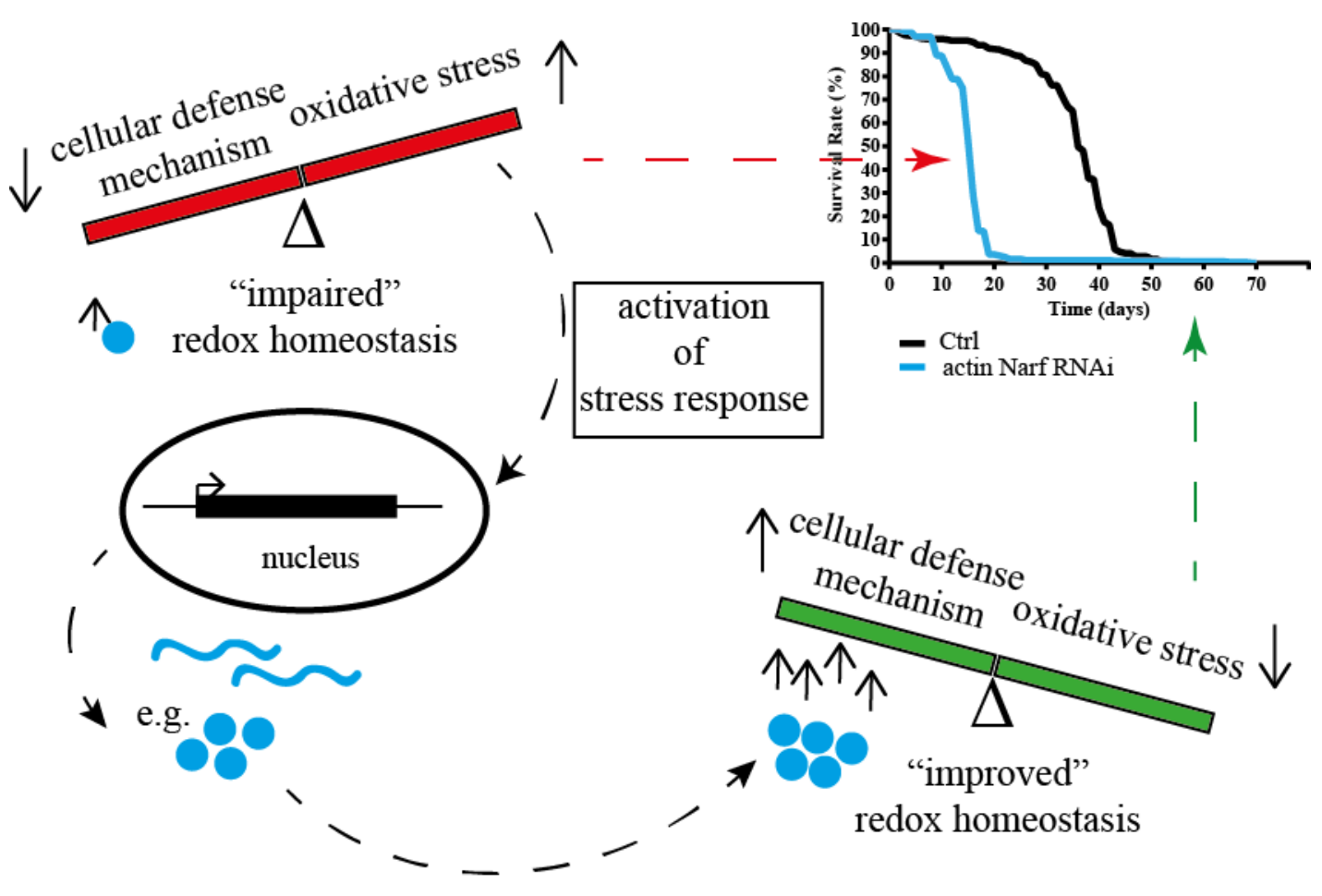

Narf protein

Figure 47: Narf down regulation might increase protein expression via a negative feedback An ubiquitous down regulation of Narf severely impaired the survival rate of the majority of the flies (red arrow). This was reasoned to an essential function of Narf during adulthood. However, a subpopulation of 
approximately 5\% survived and even showed a prolongation in lifespan (green arrow). Furthermore a general increase in the Narf protein level was observed upon down regulation. I hypothesize that a ubiquitous knock down of Narf caused a severe stress situation, which led to the activation of a response signaling pathway and by this increased Narf protein levels via a negative feedback loop in order to maintain homeostasis. If this effect is mediated on the DNA, mRNA or protein level is not known. The fact that Narf overexpression achieved an increase in lifespan is consistent to the observed lifespan extension upon overexpression of genomic and inducible transgenes. Thus, Narf might be a new regulator involved in stress response and by this involved in aging processes.

\subsubsection{An altered Narf expression in muscle cells probably leads to redox imbalance}

Muscle specific experiments showed a decrease in lifespan of approximately 25\% in a Narf knock down situation (Figure 34) and a decrease of approximately 20\% in Narf overexpressing situations induced with $20 \mu \mathrm{M}$ of RU. An increase in gene expression by using $200 \mu \mathrm{M}$ of RU further shortened lifespan (Figure 32). Next to this, aging phenotypes such as nuclear morphology changes and a decrease in climbing behavior was observed upon muscle specific overexpression of Narf (WT or H/R) (Figure 29, Figure 30).

However, the biggest problems using phenotypic analyses as a tool to study aging is that a distinct effect might be observed but the molecular reasons behind remain unknown. Due to this RNA sequencing analyses were performed to study changes in gene expression in thorax samples upon altered Narf expression in muscle cells (Figure 36). A comparison of the RNA sequencing analyses showed an overlap in gene expression changes. In case of the overexpressed Narf wild type transgene 177 genes were identified for being changed. Out of these approximately $50 \%$ of the candidates were also found upon overexpression of the mutant Narf H/R transgene as well as upon Narf down-regulation. The most interesting candidates were selected based on the logefold change on gene expression level. Methuselah-like 8 (log 2 fold: + 7.11) and Cyp12d1 (log 2 fold: - 6.58) were identified when Narf was muscle-specific down regulated; whereas Cyp4p2 (log 2 fold: +4.59 WT, +4.64 H/R) was detected upon Narf overexpression.

Methuselah-like 8 is annotated to be involved in aging in Drosophila due to its homology with methuselah (mth) which mutant could cause a lifespan extension (Lin et al., 1998). It was shown that methuselah increases lifespan accompanied by a resistance to several kinds of stress including heat, starvation, and oxidative stress (Lin et al., 1998). It was suggested that the wild type methuselah allele has a central role in maintaining homeostasis and metabolism by modulating molecular events in response to stress and that the level of gene 
expression plays a critical role in balancing the system (Lin et al., 1998). Methuselah has 15 paralogs, namely methuselah-like 1-15 which functions are largely unknown (Mendoza et al., 2016). There are no evidences that they exhibit the same function (Araujo et al., 2013). Nevertheless, the changes in methuselah-like 8 expression level indicate that a Narf knockdown is inducing stress which might be the reason for the observed shortened lifespan. Furthermore, the gene expression of Cyp12d1 was changed in thorax samples upon Narf down regulation. Cyp12d1 belongs to the group of cytochrome $\underline{\mathrm{P}} 450$ monooxygenases (P450s) which is large, conserved and able to catalyze a variety of enzymatic reactions (reviewed in Sono et al., 1996 and Scott et al., 2001). In Drosophila 83 P450 genes exist, which are involved in essential processes as well as in adaptive response to chemical compounds (Tijet et al., 2001). However, the function of Cyp-proteins in insects is not well understood yet. The expression pattern of P450s in Drosophila has been studied. It has been shown by performing an RNAi lethality screen that P450s are needed for essential processes which are potentially involved in detoxification (Chung et al., 2009). Thus, 35 P450s genes were identified for being putatively involved in the cellular detoxification system including Cyp6a8, Cyp6w1 and Cyp12d1d which were identified for being changed in their expression profile upon Narf down regulation in this study.

An interesting observation was that a muscle specific overexpression of the two Narf alleles (wild type and mutant H/R) also led to changes in gene expression for P450 monoxygenases (like Cyp4p2 and Cyp4p3). Next to the P450s also GST- (GstE14) and UGT-proteins (Ugt36Bb) were found to be changed. These genes are involved in all three phase of the detoxification system comprising phase I detoxification enzymes especially P450, phase II enzymes such as glutathione S-transferases and phase III enzymes including UDP-glucoronosyltransferases (Misra et al., 2011) have been found to be changed in expression level upon altered Narf expression in muscle cells. The Nuclear factor erythroid 2-related factor 2 (Nrf2) was described for being a transcription factor involved in redox balance by controlling gene expression of detoxifying genes. Nrf2 is associated to the Kelchlike ECH associated protein 1 (Keap-1) which mediates Nrf2 ubiquitinylation followed by degradation (reviewed in Motohashi and Yamamoto, 2004 and Loboda et al., 2016). However, under stressed situations Keap-1 is oxidized which disturbs Nrf2 binding. Thus, Nrf2 translocates to the nucleus and activates the cellular defense mechanism by binding to the ARE sequence (Jain et al., 2010). The Nrf2 transcription factor is quite conserved and can be also found in Drosophila where it is called Cap-and-collar C (CncC). A first link of 
CncC/Nrf2 to the nuclear lamina in Drosophila was made by Dialynas et al., in 2015. There it was shown that a muscle specific overexpression of mutant lamins activated the Nrf2(CncC)/Keap-1 pathway during larval stage by causing reductive stress. Nrf2 mediated gene expression is different in oxidative and reductive stress. Reductive stress prevents Nrf2Keap1 association by direct competition with p62/SQSTM1-Keap1 association (Komatsu et al., 2010). p62/SQSTM1 is also conserved and be can be found in Drosophila named as p62/Ref(2)P. It was shown that in mutant lamin expressing larvae as well as in muscular dystrophy affected human patients the protein level for p62 is increased (Dialynas et al., 2015).

In order to analyze if p62/Ref(2)P protein level is elevated upon muscle specific alteration in Narf expression western blot analyses of thorax samples were performed. However, an increase in p62/Ref(2)P protein level was not observed after 17 days of induction (Supplement Figure 7). This might indicate that Narf is not inducing reductive stress but do not exclude the activation of the Nrf2 signaling pathway by oxidative stress. Another hint showing that the Nrf2 pathway might be activated is the observed change in gene expression for Nrf2/CncC target genes.

Misra et al., 2011 analyzed gene expression profile upon xenobiotic exposure in Drosophila and identified its target genes. A Comparison of these data with the total list of identified RNA sequencing candidates after muscle specific Narf-RNAi or Narf OE showed that in all cases approximately 15\% of the candidates were described for being CncC/Nrf2 dependent. I hypothesize that the muscle specific alteration of Narf expression could cause an imbalance in redox homeostasis, which lead to the activation of the Nrf2/Keap-1 signaling pathway as a mechanism to defense the stress situation with the observed activation in gene expression. However, it was already reported that $\mathrm{CncC}$ is involved in aging due to a decreased ability of target gene expression upon stress in aged flies, suggesting that a constitutional activation of the Nrf2/Keap-1 pathway leads to an exhaustion over time (Rahman et al., 2013).

Consistent to this it might be reasonable that an altered Narf expression induces stress, which causes a constitutional activativation of the Nrf2/Keap1 signalling pathway and finally results in a shortened lifespan which might represent an accelerated aging effect. 


\subsubsection{The human mutation might represent a loss-of function allele}

Considering the current knowledge about Narf it seems that the human mutation represents a loss of function allele. The strongest argument for this hypothesis was the detected lifespan prolongation in Drosophila upon overexpression. This effect was observed in two independent experiments first by an increase in genomic Narf alleles (Figure 24 B) and second by a tubulin-Gal4 controlled gene expression (Figure 25). Survival rate analyses confirmed that the development of Narf overexpressing flies was not affected, showing that lifespan analyses were performed with representative adults (Figure 24 A). That a gain of function might be beneficial was further indicated by the elevated Narf protein level upon ubiquitous down regulation (Figure $35 \mathrm{~B}$ ), which was probably responsible for the increase in lifespan of a distinct subpopulation of flies (Figure $35 \mathrm{~A}$ ).

A mutant version of the C.elegans homologue oxy-4 was identified for being more sensitive to oxidative stress and showing a decrease in lifespan (Fujii et al., 2009). An interesting fact is that the mutation refers to the homologous position of the human patient. It was described that oxy-4 mutants show a delay in development of 1 to 1.5 days compared to control worms (Fujii et al., 2009). Similar results have been observed upon RNAi treatment (Figure 38), suggesting delayed development is a loss-of function phenotype. However, a decrease in lifespan in RNAi worms was not observed, probably due to a sufficient protein expression level upon inefficient down regulation (Figure 44).

However, an important point which should not be left out of consideration is the fact that in humans two homologues namely Narf (IOP-2) and Narf-like (IOP-1) exist, whereas only one can be found in invertebrates like CG17683 (Narf) in Drosophila and Oxy-4 in C.elegans. It has been shown in this study that the Drosophila homologue localizes to the nuclear envelope as well as to the cytoplasm (Figure 18). Compared to this is the human Narf restricted to the nuclear envelope whereas IOP-1 is predominantly cytoplasmatic localized (Barton and Worman 1999, Song and Lee 2008). These data suggest that Drosophila Narf takes over functions which are separated in vertebrates. To better understand the molecular nature of the human Narf mutation and its role in aging it will be necessary to further study Drosophila Narf gene function and its involvement in signaling pathways. 


\section{Materials and Methods}

\subsection{Materials}

\subsubsection{General Buffers}

Table 1: List of buffers

\begin{tabular}{|l|l|}
\hline \multicolumn{1}{|c|}{ Agent } & \multicolumn{1}{|c|}{ Ingredients } \\
\hline \hline PBS (1x) & $\begin{array}{l}137 \mathrm{mM} \mathrm{NaCl,} \mathrm{10} \mathrm{mM} \mathrm{Na} \mathrm{mPO}_{4}, 2.7 \mathrm{mM} \mathrm{KCL,} \mathrm{1.8} \mathrm{mM} \\
\mathrm{KH}_{2} \mathrm{PO}_{4}, \text { [pH 7.4], 1l }\end{array}$ \\
\hline Sample buffer (2x) & $\begin{array}{l}100 \mathrm{mM} \text { Tris-Cl [pH 6.8], 4\% SDS, 0.2\% bromphenolblue, } \\
\text { 20\% glycerol, 200 mM DTT }\end{array}$ \\
\hline SDS-Page buffer (10x) & $0.25 \mathrm{M}$ Tris, 2 M glycine, 1\% SDS, 1l \\
\hline TAE-buffer (50x) & 2 M Tris, 50 mM EDTA, 1 M acetic acid [pH 8.5], 1l \\
\hline Transfer-buffer (1x) & $193 \mathrm{mM}$ glycine, 25 mM Tris, 5\% methanol, 1l \\
\hline
\end{tabular}

\subsubsection{Chemical reagents}

All chemicals used in this study were purchased from AppliChem GmbH (Darmstadt, Germany), Carl ROTH (Karlsruhe, Germany) or Sigma-Aldrich (Steinheim, Germany) unless otherwise stated.

\subsubsection{Laboratory services}

BestGene (Chino Hills, CA, US)

BioScience (Göttingen, Germany)

Charles River (Wilmington, Massachusetts, US)

Genetivision (Houston, Texas, US)

Hybrigenics (Paris, France)

InDroso (Rennes, France)

\subsubsection{Antibiotics}

$\begin{array}{lll} & \text { Stock concentration } & \text { Working concentration } \\ \text { Ampicillin } & \left(100 \mathrm{mg} / \mathrm{ml} \text { in } \mathrm{ddH}_{2} 0\right) & 50-100 \mu \mathrm{g} / \mathrm{ml} \text { (final) } \\ \text { Kanamycin } & \left(10 \mathrm{mg} / \mathrm{ml} \text { in } \mathrm{ddH}_{2} 0\right) & 50 \mu \mathrm{g} / \mathrm{ml} \text { (final) }\end{array}$




\subsubsection{Enzymes and Kits}

All restriction enzymes used in this study were either purchased from Fermentas/Thermo Fisher scientific (Waltham, USA) or New England Biolabs GmbH (Ipswich, USA).

Enzymes from other sources are listed below.

DNaseI

Long PCR Enzyme Mix

Lysozym

M-MLV Reverse transcriptase

PfuS mix DNA Polymerase

Proteinase K

RQ1 RNase-free DNase

T4 DNA Ligase

T4 DNA Polymerase

Taq DNA Polymerase
Roche (Basel, Swiss)

Fermentas

AppliChem (Darmstadt, Germany)

Promega (Madison, USA)

expressed and purified in the Großhans lab

Roche

Promega

Fermentas

Fermentas

expressed and purified in the Großhans lab

Following Kits were used as described in manufacturer`s instructions.

Arcturus $^{\mathrm{TM}}$ PicoPure ${ }^{\mathrm{TM}}$ RNA isolation Kit, Thermo Fisher scientific

In-fusion HD cloning kit, Clontech (Mountain View, USA)

MiniElute Gel extraction Kit, Qiagen (Hilden, Germany)

Plasmid Midi Kit Nucleobond Xtra, Macherey-Nagel (Düren, Germany)

Presto $^{\mathrm{TM}}$ Mini Palsmid Kit, Geneaid (New Taipei City, Taiwan)

PureLink ${ }^{\mathrm{R}}$ PCR-Purification Kit, Invitrogen (Carlsbad, USA)

SYBR ${ }^{\circledR}$ FAST qPCR Kit Master Mix (2X) Universal, KapaBiosystems

\subsubsection{Chromatography}

CNBr activated Sepharose 4B, GE Healthcare Life Sciences (Little Chalfont, UK)

Dynabeads MyOne Streptavidin T1 beads, Life technologies (Carlsbad, USA)

Ni-sepharose beads, Amersham Pharmacia Biotech (Little Chalfont, UK)

PD-10 desalting columns, GE Healthcare Life Sciences 


\subsubsection{Fly stocks}

Table 2: Provided flystrains

\begin{tabular}{|c|c|c|}
\hline Name & Genotype & $\begin{array}{l}\text { Source/Lab stock } \\
\text { number }\end{array}$ \\
\hline \multicolumn{3}{|c|}{ A) General strains: } \\
\hline OregonR & $+/+$ & A401 \\
\hline w1118 & $\mathrm{W}$ & A101 \\
\hline $\mathrm{Tft}$ & w ; Tft, c px sp / CyO & A207 \\
\hline BL25709 & $\begin{array}{l}\mathrm{y}^{1} \mathrm{v}^{1} \mathrm{P}\{\text { nos- } \\
\text { phiC31 }\end{array}$ & Bloomington \\
\hline \multicolumn{3}{|l|}{ B) Driverlines: } \\
\hline actin-Gal4 & w ; actin-Gal4 $\left\{\mathrm{w}^{+}\right\} / \mathrm{CyO}$ & $\begin{array}{l}\text { N. Lee/ } \\
\text { B118 }\end{array}$ \\
\hline actinGS-Gal & $\begin{array}{l}\text { y w Flp[122]\{ry+\} ; UAS-GFP / CyO ; } \\
\text { act-Gal4-PR[3] / TM6B, Tb Hu }\end{array}$ & $\begin{array}{l}\text { Bloomington/ } \\
\text { B121 }\end{array}$ \\
\hline armadillo-Gal4 & arm-GAL4, UAS-lacZ & $\begin{array}{l}\text { Wieschaus/ } \\
\text { B111 }\end{array}$ \\
\hline GMR-Gal4 & w ; Bcg/CyO; GMR-Gal4\{w+\} & B104 \\
\hline MHC-GS-Gal & w ; GS-Gal4-Switch[MHC] $\left\{\mathrm{w}^{+}\right\}$ & $\begin{array}{l}\text { Helfand/ } \\
\text { B127 }\end{array}$ \\
\hline tubulin-Gal4 & w ; tubulin-Gal4\{w+\}LL7 / TM3, Sb & $\begin{array}{l}\text { N. Lee/ } \\
\text { B119 }\end{array}$ \\
\hline vestigial-Gal4 & $\mathrm{w}$; vestigial-Gal $4\left\{\mathrm{w}^{+}\right\}$ & B108 \\
\hline \multicolumn{3}{|c|}{ C) Kugelkern concerning strains: } \\
\hline kuk & $\begin{array}{l}\text { w ; dur }+\left\{w^{+}\right\}, \operatorname{kuk} 15 \text { e ca } / \operatorname{dur}+\left\{w^{+}\right\} \\
\text {Df(3R)EX6176 }\left\{w^{+}\right\} \text {e ca }\end{array}$ & F026 \\
\hline kukD15 ( $\Delta 15)$ & $\begin{array}{l}\text { w ; Sp / CyO, hb-lacZ }\{\text { ry }+\} ; \operatorname{dur}+\left\{\mathrm{w}^{+}\right\} \\
\text {kuk15 е са }\end{array}$ & F030 \\
\hline UASt-Kuk & w ; UASt-kuk $\{\mathrm{w}+\} / \mathrm{CyO}, \mathrm{Dr} / \mathrm{Tm} 3$, Ser & $\begin{array}{l}\text { H.Steffen/ } \\
\text { F122 }\end{array}$ \\
\hline GFP-Kuk; D15 & $\begin{array}{l}\text { w ;matGal4(67) }\left\{\mathrm{w}^{+}\right\} \text {, UASp-GFP }\left\{\mathrm{w}^{+}\right\} \\
/ \text {CyO; dur }+\left\{\mathrm{w}^{+}\right\}, \operatorname{kuk} 15 \text { e ca } / \operatorname{dur}+\left\{\mathrm{w}^{+}\right\} \\
\text {Df(3R)EX6176 }\left\{\mathrm{w}^{+}\right\} \text {e ca }\end{array}$ & F113 \\
\hline \multicolumn{3}{|c|}{ D) Narf concerning strains: } \\
\hline 7172 & CG17683 ${ }^{\mathrm{NC} 37} \mathrm{cn}^{1} \mathrm{bw}^{1} / \mathrm{SM} 1$ & Bloomington \\
\hline 7173 & $\mathrm{CG} 17683^{\mathrm{NC} 38} \mathrm{cn}^{1} \mathrm{bw}^{1} / \mathrm{SM} 1$ & Bloomington \\
\hline 7176 & $\mathrm{CG} 17683^{\mathrm{NC} 70} \mathrm{cn}^{1} \mathrm{bw}^{1} / \mathrm{SM} 1$ & Bloomington \\
\hline 7162 & 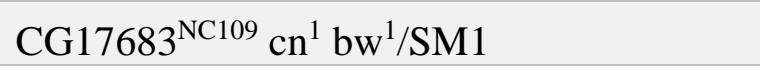 & Bloomington \\
\hline v110003/Narf-RNAi & $\mathrm{P}\{\mathrm{KK} 115708\} \mathrm{VIE}-260 \mathrm{~B}$ & VDRC \\
\hline \multicolumn{3}{|c|}{ E) Other fly strains } \\
\hline twistGFP, Cy0 & w; Gla/Cy0,\{twi::GFP\} & $\begin{array}{l}\text { B. Shiloh, } \\
\text { Weizmann Institute } \\
\text { of Science (Israel) }\end{array}$ \\
\hline
\end{tabular}




\begin{tabular}{|l|l|l|}
\hline UAS-GFP & w; UASt-GFP $\left\{\mathrm{w}^{+}\right\}$ & $\begin{array}{l}\text { Paros/ } \\
\text { B204 }\end{array}$ \\
\hline GFP-cadherin & w; ubi-DE-cadherin-GFP $\left\{\mathrm{w}^{+}\right\}$ & $\begin{array}{l}\text { T. Lecuit/ } \\
\text { B312 }\end{array}$ \\
\hline \multicolumn{2}{|c|}{ F) Fly strains used in RNAi-Screen (see Supplement Table 2) } \\
\hline
\end{tabular}

Table 3: Flystrains generated in this study

\begin{tabular}{|c|c|c|}
\hline Name & Integration & Injection \\
\hline GFP-Kuk & $\begin{array}{l}2 / 3 \text { transposase mediated integration } \\
4^{\text {th }} \text { chromosome }\end{array}$ & M. Kriebel \\
\hline GFP-Kuk & $\begin{array}{l}\Delta 2 / 3 \text { transposase mediated integration } \\
2^{\text {nd }} \text { chromosome }\end{array}$ & GenetiVision \\
\hline KukC567S & $\begin{array}{l}\Delta 2 / 3 \text { transposase mediated integration } \\
2^{\text {nd }} \text { chromosome }\end{array}$ & GenetiVision \\
\hline GFP-KukC567S & $\begin{array}{l}\Delta 2 / 3 \text { transposase mediated integration } \\
2^{\text {nd }} \text { chromosome }\end{array}$ & GenetiVision \\
\hline pAttP Narf genomic & BL25709 (2L) 25C6 & M. Kriebel \\
\hline pAttP Narf genomic & VK1(2R) 59D3 & GenetiVision \\
\hline pAttP Narf genomic & P2(3L) 68A4 & GenetiVision \\
\hline pUASt Narf & BL25709 (2L) $25 \mathrm{C} 6$ & M. Kriebel \\
\hline pUASt Narf H/R & BL25709 (2L) 25C6 & M. Kriebel \\
\hline
\end{tabular}

\subsubsection{Worm strains and RNAi-vectors used in this study}

Table 4: Worm strains used in this study

\begin{tabular}{|l|l|l|}
\hline \multicolumn{1}{|c|}{ Name } & \multicolumn{1}{|c|}{ Genotype } & \multicolumn{1}{c|}{ Source } \\
\hline \hline N2 & wild type & Gruenbaum lab \\
\hline qa5001(oxy-4) & D373N substitution & M. Fujii \\
\hline GFP::Ce-lamin & baf-1p-gfp::lmn-1 & Gruenbaum lab \\
\hline
\end{tabular}

Table 5: RNAi-vectors used in this study

\begin{tabular}{|l|l|l|}
\hline \multicolumn{1}{|c|}{ Name } & \multicolumn{1}{|c|}{ Genotype } & \multicolumn{1}{c|}{ Source } \\
\hline \hline L4440 & Empty vector RNAi & Gruenbaum lab \\
\hline Imn-1 RNAi & RNAi against Ce-Lamin & Gruenbaum lab \\
\hline Y54H5A.4 & RNAi against Oxy-4 & ORF RNAi Ressource - VIDAL \\
\hline
\end{tabular}




\subsubsection{Media for flies}

\section{Fly food}

$64 \mathrm{~g}$ of thread agar were cooked in seven liter water until agar was completely dissolved. Afterwards $0.64 \mathrm{~g}$ maize meal, 80 g soya-bean meal, $200 \mathrm{~g}$ of baker`yeast, $640 \mathrm{~g}$ malt extract and $175 \mathrm{~g}$ sugar-beet molasses were added and properly mixed. The food was cooked for another $30 \mathrm{~min}$ and cooled down afterwards to $55-60^{\circ} \mathrm{C} .12 \mathrm{~g}$ Nipagin dissolved in $90 \mathrm{ml}$ ethanol and $75 \mathrm{ml}$ propionic acid were finally added. Food was mixed again and filled in fly vials. The food was kept overnight at room temperature to cool down and to solidify. Vial plugging was performed the next day. Fly food vials were stored at $18^{\circ} \mathrm{C}$ until usage.

\section{Apple juice agar plates}

To prepare one liter of apple juice agar, $17.5 \mathrm{~g}$ agar were dissolved in $750 \mathrm{ml}$ water. After autoclaving a mix of $25 \mathrm{~g}$ sugar solved in $250 \mathrm{ml}$ of apple juice and $1.5 \mathrm{~g}$ of Nipaging dissolved in $10 \mathrm{ml}$ ethanol was added to the agar solution. The mixture was allowed to cool down to $60{ }^{\circ} \mathrm{C}$ and apple juice agar was poured to Petri dishes. Plates were kept at $4{ }^{\circ} \mathrm{C}$.

\subsubsection{Media for C.elegans}

\section{NGM plates}

To prepare the standard nematode growth medium, $3 \mathrm{~g} \mathrm{NaCl}, 17 \mathrm{~g}$ agar and $2.5 \mathrm{~g}$ peptone were mixed in an Erlenmeyer flask and dissolved in $975 \mathrm{ml}$ of water. Suspension was autoclaved for $50 \mathrm{~min}$ and after cooling down to $55{ }^{\circ} \mathrm{C}, 1 \mathrm{ml} \mathrm{CaCl} 2(1 \mathrm{M}), 1 \mathrm{ml}$

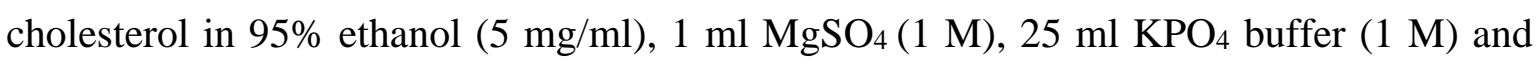
streptomycin (300 ng/ml) were added. The medium was mixed and dispensed into Petri plates by using a peristaltic pump under steril conditions. Plates have been dried at room temperature for 2 - 3 days and finally stored at $4^{\circ} \mathrm{C}$.

\section{RNAi plates}

The same protocol for NGM plates was used but streptomycin was replaced by $50 \mu \mathrm{g} / \mathrm{ml}$ ampicillin and $120 \mu \mathrm{g} / \mathrm{ml}$ IPTG. 


\section{Seeding plates}

$5 \mathrm{ml}$ starter culture of bacteria (OP50 or RNAi expressing bacteria) was set up in LB medium by picking a single colony and incubated for overnight, best $8 \mathrm{~h}$, at $37^{\circ} \mathrm{C} .50-100 \mu \mathrm{l}$ were pipetted under sterile conditions to either NGM or RNAi plates and distributed equally. The plates were let dry over night at room temperature and finally shifted to $4{ }^{\circ} \mathrm{C}$ for storage.

\subsubsection{Bacterial cell lines}

Following E.coli strains were used in this study:

DH5 $\alpha$ was used for plasmid amplification.

F-, ø80dlacZAM15, $($ (lacZYA-argF)U169, deoR, recA1, endA1, hsdR17(rK -,mK+), phoA, supE44, $\lambda-$-thi-1, gyrA96, relA1

BL21DE3(rosetta) was used for protein expression.

$\mathrm{F}^{-}$ompT hsdS $\mathrm{B}_{\mathrm{B}}\left(\mathrm{rB}^{-} \mathrm{mB}^{-}\right) \mathrm{gal} d c m(\mathrm{DE} 3)$ pRARE $\left(\mathrm{Cam}^{\mathrm{R}}\right)$

OP50 was used for seeding NGM-plates.

[ura-,strR,rnc-,(delta)attB::FRT-lacl-lacUV5p-T7]

\subsubsection{Media for bacterial culture}

\section{LB-medium}

To make one liter of LB-medium, $10 \mathrm{~g}$ Bactotryptone, $5 \mathrm{~g}$ Yeast extract and $10 \mathrm{~g}$ Sodium chloride were dissolved in water, autoclaved and stored at $4{ }^{\circ} \mathrm{C}$.

\section{LB-plates}

LB-plates additionally contained $10 \mathrm{~g}$ agar and a desired antibiotic which was added after LB-solution was cooled down to $55^{\circ} \mathrm{C}$. LB-plates were poured into Petri dishes and stored at $4{ }^{\circ} \mathrm{C}$.

\subsubsection{Oligonucleotides}

All oligonucleotides named by numbers were ordered from Eurofins Genomics (Germany). Alphabetically named oligonucleotides were ordered from hylabs (Israel). 
Bold letters within oligonucleotide sequence show restriction enzyme sites, big letters were used for vector sequences within infusion primers.

Table 6: Oligonucleotides used for C.elegans studies

\begin{tabular}{|c|c|c|}
\hline Name & Sequence $5^{`} \rightarrow 3^{`}$ & Description \\
\hline MKa & taatagggcccatatttgtatagttcatccatgcc & $\begin{array}{l}\text { Amplification of vectorbackbone } \\
\text { Baf1PGFP lmna without Imna } \\
\text { inclusive Bsp120I site }\end{array}$ \\
\hline MKb & atatagttaacctctagaactagtggatccc & $\begin{array}{l}\text { Amplification of vectorbackbone } \\
\text { Baf1PGFP lmna without lmna } \\
\text { inclusive HpaI sites }\end{array}$ \\
\hline MKc & gataagggcccatggaagattctggattcagtgg & $\begin{array}{l}\text { Amplification genomic oxy-4 inclusive } \\
\text { Bsp120I site }\end{array}$ \\
\hline MKd & agatagttaactcaccatttcaagacttgagcaac & $\begin{array}{l}\text { Amplification genomic oxy-4 inclusive } \\
\text { HpaI site }\end{array}$ \\
\hline MKe & tgtagaatccgaagctggag & Sequencing for $\mathrm{D} / \mathrm{N}$ \\
\hline MKf & actggagaattaacagacgtc & Sequencing for in frame GFP to oxy-4 \\
\hline MKg & tggattcagtggtgtcgttcgac & qPCR Primer oxy-4 (Primermix 1) \\
\hline MKh & ccactacatgccagacaatcagc & qPCR Primer oxy-4 (Primermix $1+4$ ) \\
\hline MKi & gaaaacgacgaagccggagatgttg & qPCR Primer oxy-4 (Primermix $2+7$ ) \\
\hline MKj & ctccagaagcaccaccatcgtc & qPCR Primer oxy-4 (Primermix 2) \\
\hline MKk & gacgatggtggtgcttctggag & qPCR Primer oxy-4 (Primermix 3) \\
\hline MKl & ctccagcttcggattctacagtcg & qPCR Primer oxy-4 (Primermix $3+7$ ) \\
\hline MKq & gctccgaatttggactgcataatcc & qPCR Primer oxy-4 (Primermix 4) \\
\hline MKr & tagctgattgtctggcatgtagtgg & qPCR Primer oxy-4 (Primermix 5) \\
\hline MKs & ggagaaacagtgacgactgagagc & qPCR Primer oxy-4 (Primermix 5) \\
\hline MKt & agagaaggtttaagcccctgtgatg & qPCR Primer oxy-4 (Primermix 6) \\
\hline MKu & caacatctccggcttcgtcgttt & qPCR Primer oxy-4 (Primermix 6) \\
\hline MK48 & gaagagcatggttatcggc & Sequencing Primer oxy-4 \\
\hline MK49 & gccgataaccattgctctc & Sequencing Primer oxy-4 \\
\hline $\operatorname{lmn}-1$ & gggtccgtgttgcaaacaactc & qPCR Primer lmn-1 (Gruenbaum lab) \\
\hline lmn-1 & ctccaattggccactgttgcttc & qPCR Primer lmn-1 (Gruenbaum lab) \\
\hline pmp-3 & cgacgattcatttggctcaacgc & qPCR Primer pmp-3 (Gruenbaum lab) \\
\hline pmp-3 & gatgtcgcgaatttgaacctggag & qPCR Primer pmp-3 (Gruenbaum lab) \\
\hline
\end{tabular}

Table 7: Oligonucleotides used for Drosophila studies

\begin{tabular}{|c|c|c|}
\hline Name & Sequence $5 ` \rightarrow 3 `$ & Description \\
\hline \multicolumn{3}{|c|}{ Oligonucleotides used for making GFP-transgenes } \\
\hline MK9 & $\begin{array}{l}\text { AAGCAAAATGGCTAGCtccaagggcga } \\
\text { ggagctg }\end{array}$ & $\begin{array}{l}\text { Amplification EGFP from Twe- } \\
\text { Vector }\end{array}$ \\
\hline MK10 & $\begin{array}{l}\text { CGCTTGTGTTGCTAGCCTTGTACA } \\
\text { GCTCgtccatgcc }\end{array}$ & $\begin{array}{l}\text { Amplification EGFP from Twe- } \\
\text { Vector }\end{array}$ \\
\hline \multicolumn{3}{|c|}{ Oligonucleotides used for $\Delta 15$ characterisation } \\
\hline MK11 & atggcgaatgcggtggtgga & PCR primer cal1 \\
\hline
\end{tabular}




\begin{tabular}{|c|c|c|}
\hline MK12 & ttgtggtgcggtggaaggtac & PCR primer cal1 \\
\hline MK13 & tggactgcacagaggttgagg & PCR primer cher \\
\hline MK14 & ctacacatcgatctggaatggg & PCR primer cher \\
\hline MK15 & atgcgtcggcggttatttatcag & PCR primer Keap1 \\
\hline MK16 & tcatgtggctgcgctcgtg & PCR primer Keap1 \\
\hline MK17 & taacatcaacagtcaggtgtggtg & PCR primer Dad \\
\hline MK18 & tcaccgcagatgactaaagtgaac & PCR primer Dad \\
\hline MK21 & taaccacgtaaatcggctggg & PCR primer kuk \\
\hline MK22 & ctaccacgcggagaaatacc & PCR primer kuk \\
\hline MK23 & gcgctaatgcgaacttggc & PCR primer kuk \\
\hline MK24 & ttggtgtatgcatgggtattgaaggc & PCR primer kuk \\
\hline MK25 & atctttccagctgttgattagagccg & PCR primer kuk \\
\hline MK26 & gaaattccaagacgtgaaccatcgtg & Long range PCR primer \\
\hline MK27 & tatggaaatggacttgagaccaaggc & PCR primer kuk \\
\hline MK28 & ccgatgaagataacgccttggctaaa & PCR primer kuk \\
\hline MK29 & tttagccaaggcgttatcttcatcgg & Long range PCR primer \\
\hline MK30 & acgaatttcttcacttcagcc & Sequencing primer \\
\hline MK31 & ctcgaacacagacttcag & Sequencing primer \\
\hline \multicolumn{3}{|c|}{ Oligonucleotides used for Narf constructs } \\
\hline MK32 & gaaaaggtatcaaattacagatttgttgaggtaatggcc & Site directed mutagenesis - Narf \\
\hline MK33 & ggccattacctcaacaaatctgtaatttgataccttttc & Site directed mutagenesis - Narf \\
\hline MK34 & cgagaagacgctatctggc & Sequencing NarfH/R cDNA clone \\
\hline MK35 & catggtaacgggtgtgcag & Sequencing NarfH/R cDNA clone \\
\hline Mk36 & cgaagttatggatcaggcc & $\begin{array}{l}\text { Sequencing NarfH/R cDNA clone } \\
\text { binds within pFLC vector }\end{array}$ \\
\hline MK37 & ccacacctatctgatagcg & Sequencing NarfH/R cDNA clone \\
\hline MK38 & cgctatcagataggtgtgg & Sequencing NarfH/R cDNA clone \\
\hline MK39 & gccagatagcgtcttctcg & Sequencing NarfH/R cDNA clone \\
\hline MK40 & ctgcacacccgttaccatg & Sequencing NarfH/R cDNA clone \\
\hline MK41 & cgaaggatcaggcccttatg & $\begin{array}{l}\text { Sequencing NarfH/R cDNA clone } \\
\text { binds within pFLC vector }\end{array}$ \\
\hline MK42 & $\begin{array}{l}\text { TTAACCATGGGAGCCtcgaggttgagca } \\
\text { gagcg }\end{array}$ & $\begin{array}{l}\text { Infusion primer, generation of a } \\
\text { Narf specific antibody }\end{array}$ \\
\hline MK43 & $\begin{array}{l}\text { GATGAGATCTGGATCgccaatttatattaa } \\
\text { gcgatatactg }\end{array}$ & $\begin{array}{l}\text { Infusion primer, generation of a } \\
\text { Narf specific antibody }\end{array}$ \\
\hline MK44 & gcgaggttaatggcgttgtagc & $\begin{array}{l}\text { Amplification Narf } \begin{array}{r}\text { genomic } \\
\text { construct inclusive } \\
\text { regions }\end{array} \\
\text { repetitive }\end{array}$ \\
\hline MK45 & cctgcgccaatggaatatttagg & $\begin{array}{l}\text { Amplification Narf } \begin{array}{r}\text { genomic } \\
\text { construct inclusive } \\
\text { regions }\end{array} \\
\end{array}$ \\
\hline MK56 & gataaggcaaggtcaaagactgg & $\begin{array}{l}\text { Sequencing primer } \\
\text { (genomic Narf + lethal alleles) }\end{array}$ \\
\hline MK57 & gagaacttgaccatgcttagctc & $\begin{array}{l}\text { Sequencing primer } \\
\text { (genomic Narf + lethal alleles) }\end{array}$ \\
\hline MK58 & ggaggagcacaaatacgtcc & $\begin{array}{l}\text { Sequencing primer } \\
\text { (genomic Narf + lethal alleles) }\end{array}$ \\
\hline
\end{tabular}




\begin{tabular}{|c|c|c|}
\hline MK59 & acgatagcttggctcactgcac & $\begin{array}{l}\text { Sequencing primer } \\
\text { (genomic Narf + lethal alleles) }\end{array}$ \\
\hline MK60 & atgcaaataagctgtgtctgcc & $\begin{array}{l}\text { Sequencing primer } \\
\text { (genomic Narf + lethal alleles) }\end{array}$ \\
\hline \multicolumn{3}{|c|}{ Oligonucleotides used for CRISPR approach } \\
\hline MK61 & ggatttatgaatggcggtaacg & Sequencing primer (putative gRNA) \\
\hline MK62 & tcaagaggcgaggttaatgg & Sequencing primer (putative gRNA) \\
\hline MK63 & aaagctagcggtggcgtaatatgcgctgc & $\begin{array}{l}\text { Cloning homology arm left, } \\
\text { inclusive NheI site }\end{array}$ \\
\hline MK64 & aaagcggccgctagtaaaagccgcgcgcgttaac & $\begin{array}{l}\text { Cloning homology arm left, } \\
\text { inclusive NotI site }\end{array}$ \\
\hline MK65 & aaaagatctactaggatttcaaaacgagtggtc & $\begin{array}{l}\text { Cloning homology arm right, } \\
\text { inclusive BglII site }\end{array}$ \\
\hline MK66 & aaaactcgagccatagtggactacaataggg & $\begin{array}{l}\text { Cloning homology arm right, } \\
\text { inclusive XhoI site }\end{array}$ \\
\hline MK67 & cttcgcgcgcggcttttactaatc & $\begin{array}{l}\text { sgRNA homology arm left } \\
\text { inclusive BbsI Restrictionsite }\end{array}$ \\
\hline MK68 & aaacgattagtaaaagccgcgcgc & $\begin{array}{l}\text { sgRNA homology arm left inclusive } \\
\text { BbsI Restrictionsite }\end{array}$ \\
\hline MK69 & cttcgccgagaaggtctgacaact & $\begin{array}{l}\text { sgRNA homology arm right } \\
\text { inclusive BbsI Restrictionsite }\end{array}$ \\
\hline MK70 & aaacagttgtcagaccttctcgg & $\begin{array}{l}\text { sgRNA homology arm right } \\
\text { inclusive BbsI Restrictionsite }\end{array}$ \\
\hline MK71 & aatcacttgaggtgaaagtgcg & $\begin{array}{l}\text { Sequencing pBFvU6.2B } \\
\text { sgHAL,sgHAR Verification of } \\
\text { sgHAL }\end{array}$ \\
\hline MK72 & gactcatcgtcccagagaatcg & $\begin{array}{l}\text { Sequencing pBFvU6.2B } \\
\text { sgHAL,sgHAR Verification of } \\
\text { sgHAR }\end{array}$ \\
\hline
\end{tabular}

\subsubsection{Plasmid constructs}

Table 8: Provided plasmids used in this study

\begin{tabular}{|c|c|c|}
\hline Name & Description & Source \\
\hline \multicolumn{3}{|c|}{ Plasmid constructs used for C.elegans studies } \\
\hline BAF-1p-GFP lamin & $\begin{array}{l}\text { GFP Ce-lamin fusion protein expressed by } \\
\text { BAF promoter }\end{array}$ & Gruenbaum Lab \\
\hline \multicolumn{3}{|c|}{ Plasmid constructs used for Drosophila studies } \\
\hline \multicolumn{3}{|c|}{ Antibodygeneration } \\
\hline pGEX6OH & $\begin{array}{l}\text { Bacterial expression, pGEX vector with } \\
\text { additional N-terminal GST tag and } \\
\text { C terminal His6 tag }\end{array}$ & Großhans lab \\
\hline pGEX6OHNup153 & $\begin{array}{l}\text { pGEX6OH digested with BamHI, } \\
\text { insertion of } 1079 \text { bp concerning Nup153 } \\
\text { (isoformA) by infusion cloning } \\
\text { (primer: MC15/MC16) }\end{array}$ & M. Clever \\
\hline
\end{tabular}




\begin{tabular}{|c|c|c|}
\hline QE80N60 & $\begin{array}{l}\text { Bacterial expression, QE60 vector with } \\
\text { additional N-terminal His6 tag }\end{array}$ & $\begin{array}{l}\text { Prof. Görlich, } \\
\text { MPI Göttingen }\end{array}$ \\
\hline \multicolumn{3}{|l|}{ CRISPR approach } \\
\hline pHD-DsRed attP & $\begin{array}{l}\text { Site specific Integration of transgenes } \\
\text { DsRed flanked by two loxPsites } \\
\text { Two MCS used for inserting homology } \\
\text { arms flanking targeted sequence }\end{array}$ & Gratz et al., 2014 \\
\hline pBFvU6.2 & sgRNA expression by U6 promoter & $\begin{array}{l}\text { Kondo and Ueda, } \\
2013\end{array}$ \\
\hline pBFvU6.2B & sgRNA expression by U6 promoter & $\begin{array}{l}\text { Kondo and Ueada, } \\
2013\end{array}$ \\
\hline \multicolumn{3}{|c|}{ Generation of transgenic flies } \\
\hline UASt attB & Site specific Integration of transgenes & Bischof et al., 2007 \\
\hline pattB & Site specific Integration of transgenes & Bischof et al., 2013 \\
\hline \multicolumn{3}{|c|}{ Other Plasmid constructs } \\
\hline RE37350 & $\begin{array}{l}\text { cDNA clone of Narf (CG17683) in pFLC } \\
\text { vector }\end{array}$ & $\begin{array}{l}\text { Flybase, } \\
\text { BDGP DGC clone }\end{array}$ \\
\hline CasBAC kuk genomic & $\begin{array}{l}\text { Genomic rescue, EcoRV fragment cloned } \\
\text { into pCasp } 4\end{array}$ & Großhans lab \\
\hline pBKS II (+) & Bluescript cloning vector & Short et al., 1988 \\
\hline CS-kug-C567S & $\begin{array}{l}\text { CG5157 cDNA from pSK (LD09231) } \\
\text { cloned into pCS2 vector via EcoRI-XhoI } \\
\text { and XhoI-XhoI }\end{array}$ & Großhans lab \\
\hline pBABR-Twine-EGFP & $\begin{array}{l}\text { Genomic DNA of twine fused to codon } \\
\text { optimized EGFP }\end{array}$ & Wischaus lab \\
\hline
\end{tabular}

Table 9: Plasmid constructs generated in this study

\begin{tabular}{|c|c|}
\hline Name & $\begin{array}{c}\text { Description } \\
\text { Source }\end{array}$ \\
\hline pFLC cDNA Narf H/R & $\begin{array}{l}\text { Introduction of mutation via site-directed- } \\
\text { mutagenesis (MK32/33), vector backbone was } \\
\text { cleaned by NotI and BamHI digest }\end{array}$ \\
\hline pUASt attB cDNA Narf & $\begin{array}{l}\text { pFLC cDNA Narf digested with BamHI for blunt } \\
\text { end cloning and NotI, pUASTattB digested with } \\
\text { XhoI for blunt end cloning and NotI }\end{array}$ \\
\hline pUASt attB cDNA Narf H/R & $\begin{array}{l}\text { pFLC cDNA Narf H/R digested with BamHI for } \\
\text { blunt end cloning and NotI, pUASTattB digested } \\
\text { with XhoI for blunt end cloning and NotI }\end{array}$ \\
\hline pAttB genomic Narf & $\begin{array}{l}\text { pAttB digested with BglII and XbaI, genomic } \\
\text { construct of CG17683 was amplified (MK44/45) and } \\
\text { digested with BglII and NheI }\end{array}$ \\
\hline pAttB genomic Narf H/R & $\begin{array}{l}\text { pAttBNarf-genomic digested with NdeI and XhoI, } \\
\text { missing Insert including AGA mutation and } \\
\text { restriction enzyme sites for NdeI and XhoI was } \\
\text { synthesized by Eurofins }\end{array}$ \\
\hline pHDdsRed attP HAL HAR & $\begin{array}{l}\text { HAL region amplified by MK63/MK64 including } \\
\text { NheI and NotI restriction enzyme sites, HAR region } \\
\text { amplified by MK65/MK66 including BglII and XhoI }\end{array}$ \\
\hline
\end{tabular}




\begin{tabular}{|l|l|}
\hline \multirow{2}{*}{ pBFvU6.2 sgRNA1 } & $\begin{array}{l}\text { restriction enzyme sites, both fragments cloned into } \\
\text { opened pHDdsRedattP vector }\end{array}$ \\
\hline pBFvU6.2B sgRNA2 & $\begin{array}{l}\text { Oligonucleotides MK67 + MK68 were annealed } \\
\text { against each other and cloned into pBFvU6.2 vector } \\
\text { via BbsI restriction enzyme site }\end{array}$ \\
\hline QE80N60 cDNA Narf & $\begin{array}{l}\text { Oligonucleotides MK69 + MK70 were annealed } \\
\text { against each other and cloned into pBFvU6.2B } \\
\text { vector via BbsI restriction enzyme site }\end{array}$ \\
\hline QE80N60 digested with BamHI, introduction of \\
amplified Narf cDNA (MK42/43) by infusion \\
cloning
\end{tabular}

\subsubsection{Primary antibodies}

\begin{tabular}{|c|c|c|c|c|}
\hline \multirow{2}{*}{$1^{\text {st }}$ Antibody } & \multirow{2}{*}{ Host } & \multicolumn{2}{|c|}{ Dilution } & \multirow{2}{*}{ Source } \\
\cline { 3 - 4 } & & Staining & Western & \\
\hline Antibodies used for C.elegans project \\
\hline Ce-LaminC (3932) & rabbit & - & $1: 100$ & $\begin{array}{c}\text { K.Wilson } \\
\text { (Gruenbaum lab) }\end{array}$ \\
\hline Myosin & mouse & - & $1: 1.000$ & Gruenbaum lab \\
\hline Phalloidin & rabbit & $1: 200$ & - & Sigma, P1951 \\
\hline
\end{tabular}

Antibodies used for Drosophila project

\begin{tabular}{|c|c|c|c|c|}
\hline Caspase & & $1: 500$ & - & AB13847, Abcam \\
\hline Dlg4 & mouse & - & $1: 100$ & DSHB \\
\hline Dm0 & mouse & $1: 200$ & $1: 1.000$ & C. Saumweber \\
\hline
\end{tabular}




\begin{tabular}{|c|c|c|c|c|}
\hline GFP & rabbit & - & $1: 6.000$ & $\begin{array}{c}\text { Sc-8334, Santa Cruz } \\
\text { Biotec. }\end{array}$ \\
\hline $\begin{array}{c}\text { Kuk } \\
\text { affinity purified }\end{array}$ & rabbit & $1: 5.000$ & $1: 10.000$ & S. Lawo/ Charles River \\
\hline Kuk & gp & $1: 5.000$ & - & $\begin{array}{c}\text { S. Lawo/ } \\
\text { Charles River }\end{array}$ \\
\hline Nup153 & mouse & - & $1: 1.000$ & AB24700, Abcam \\
\hline P62/Ref(2) & rabbit & & $1: 3.000$ & G. Juhasz \\
\hline Slam & rabbit & $1: 5.000$ & - & Charles River \\
\hline Tubulin & mouse & - & $1: 50.000$ & T5168,Sigma \\
\hline Antibodies generated during this study & gp & $1: 500$ & $1: 10.000$ & M.Kriebel/Bioscience \\
\hline Narf (polyclonal) & rabbit & Tested but unspecific & M.Kriebel/Bioscience \\
\hline $\begin{array}{c}\text { Narf (polyclonal) } \\
\text { Nup153 (polyclonal) }\end{array}$ & gp & $1: 5.000$ & $1: 10.000$ & M.Kriebel/Charles River \\
\hline $\begin{array}{c}\text { Nup153 (polyclonal) } \\
\text { affinity purified }\end{array}$ & rabbit & $1: 500$ & $1: 10.000$ & M.Kriebel/Charles River \\
\hline
\end{tabular}

\subsubsection{Secondary antibodies}

\begin{tabular}{|c|c|c|c|c|}
\hline \multirow{2}{*}{$2^{\text {nd }}$ Antibody } & \multirow{2}{*}{ Host } & \multicolumn{2}{|c|}{ Dilution } & \multirow{2}{*}{ Source } \\
\hline & & Staining & Western & \\
\hline $\begin{array}{c}\text { Anti-Rabbit } \\
\operatorname{IgG}(\mathrm{H}+\mathrm{L}) \mathrm{Cy} 3\end{array}$ & goat & $1: 200$ & - & $\begin{array}{c}\text { Jackson } \\
111-166-003\end{array}$ \\
\hline Alexa Fluor 488 & goat & $1: 500$ & - & $\begin{array}{l}\text { Molecular Probes, } \\
\text { Invitrogen }\end{array}$ \\
\hline Alexa Fluor 568 & goat & $1: 500$ & - & $\begin{array}{l}\text { Molecular Probes, } \\
\text { Invitrogen }\end{array}$ \\
\hline Alexa Fluor 647 & goat & $1: 500$ & - & $\begin{array}{l}\text { Molecular Probes, } \\
\text { Invitrogen }\end{array}$ \\
\hline $\begin{array}{c}\text { Alexa Fluor } \\
\text { Phalloidin } 488\end{array}$ & & $1: 1.000$ & - & $\begin{array}{l}\text { Molecular Probes, } \\
\text { Invitrogen }\end{array}$ \\
\hline IRDye-680RD & goat, donkey & - & $1: 20.000$ & $\begin{array}{c}\text { LI-COR } \\
\text { Biotechnology }\end{array}$ \\
\hline IRDye-800CW & goat, donkey & - & $1: 20.000$ & $\begin{array}{c}\text { LI-COR } \\
\text { Biotechnology }\end{array}$ \\
\hline
\end{tabular}




\subsubsection{Software}

Adobe Illustrator CS6, Adobe

Adobe Photoshop CS6, Adobe

Citavi4, Swiss Academic Software GmbH

GraphPad Prism6, GraphPad Software, Inc.,

ImageJ, Wayne Rasband

Image Studio, LI-COR Biosciences

IntasGDS, Intas Science Imaging

Lasergene, GATC biotech

Microsoft Excel 2013, Microsoft

Microsoft Word 2013, Microsoft

NanoDrop 2000, Thermo Fischer Scientific

OASIS, Yang et al., 2011

Rotor-Gene 6000 Series 1.7, Quiagen

SeqBuilder, DNASTAR

SnapGene, GSL Biotech LLC

UNICORN, GE healthcare

Zen 2012, Carl Zeiss

\subsubsection{Equipment}

Microscopes:

Axioplan 2 Fluorescence Microscope, Zeiss

LSM 780, Zeiss

MZ12.5, Leica

PrimoVert Inverted Microscope, Zeiss

Stemi 2000 Stereomicroscope, Zeiss

SteREO Lumar V12, Zeiss

SZX12 Fluorescence Stereo Microscope, Olympus

\section{Camera:}

AxioCam MRc, Zeiss 
Other Equipment:

Äkta pure, GE healthcare

Centrifuges, Eppendorf

ChemiDoc, Biorad

Concentrator, Eppendorf

DynaMagnet, life technologies

Electrophoresis Constant Power Suppley, Pharmacia

FemtoJet, Eppendorf

Flaming/Brown Micropipette Puller P97, Sutter Instrument

High Pressure Homogenizer/Mickrofluidizer- EmulsiFlex-C5, Avestin

Megafuge, Heraeus

NanoDrop 2000c, Thermo Scientific

Odyssey CLx Infrared imaging system, LI-COR Biosciences

Real time PCR machine - rotor gene 6000, Corbett/Quiagen

Thermomixer, Eppendorf

Trans-Blot Semidry, Biorad

Ultrasonics Sonifier-450, Branson

\subsubsection{Other materials}

Agarose, Invitrogen

Aquapolymount, Polysciences, Inc.

BSA/Albumin Fraktion V, Roth

Cover Slides, Thermo Scientific

DanKlorix

DAPI, Sigma-Aldrich

Dialysis sleeve, Trans Roth E656.1 MWOC 3,500 Da

DNA ladder (1 kb), Thermo Scientific

dNTPs, Thermo Scientific

Falcon tubes (15 ml, $50 \mathrm{ml}$ ), BD Falcon

Filter papers (Ø $110 \mathrm{~mm}$ ) Macherey-Nagel

Fly cages (Ø $50 \mathrm{~mm}$, Ø $94 \mathrm{~mm}$ )

Fly vials, Greiner

Forceps 
Formaldehyd (37\%), Sigma-Aldrich

Gelatin, Dicto Bacto, 0143-02 1/4 lb

Glass homogenizer, B. Braun Biotech International

Glass pipettes (5 ml - $25 \mathrm{ml}$ ), Silber Brandt

Glass slides, Thermo Scientific

Green Taq buffer (10x), Thermo Scientific

Halocarbon oil 700, Sigma-Aldrich

Immersol 518F/W, Zeiss

Microinjection needles TW100F-4, WPI

Mifepristone (RU468), Sigma-Aldrich

Milk powder, Sucofin

Parafilm M, Bemis

Pasteur pipettes, Brandt

Petri dishes, Greiner

Phenol/Chloroform/Isopropanol, Roth

Phusion HF buffer (5x), Thermo Scientific

Pipetman ( $2 \mu \mathrm{l}, 10 \mu \mathrm{l}, 200 \mu \mathrm{l}, 1000 \mu \mathrm{l})$, Gilson

Pipette tips, Sarstedt

Platinum wire

Poly-L-Lysine Solution (0.1\%), Sigma-Aldrich

Polypropylene column, Quiagen

Prestained Protein Ladder, Thermo Scientific

Protease Inhibitor Cocktail - Complete Mini (EDTA free), Roche

Proteinase K, Roche

Protran Nitrocellulose Membrane, Amersham

Reaction tubes (1.5 ml, $2.0 \mathrm{ml})$, Sarstedt

Reaction tubes (5.0 ml), Eppendorf

Reaction tubes $(50 \mu \mathrm{l})$, Brand

Reaction tubes (500 $\mu \mathrm{l})$, ELKay

Sodium Hypochlorite solution (15\%), Sigma-Aldrich

TRI-reagent, Sigma-Aldrich

TritonX-100, Roth

Tween20, Roth 
Viva spin, Sartorius

VoltaLef Oil 10s, Lehmann, Voss \& Co.

Weigh Boat, VWR

Whatman $3 \mathrm{~mm}$ blotting paper, GE healthcare

Yeast, Dr. Oetker

\title{
6.2 Methods
}

Standard procedures were carried out, according to the protocols described in Sambrook and Russels, 2001 and to established protocols of the Großhans laboratory, unless otherwise stated.

\subsubsection{DNA methods}

\subsubsection{Extraction of genomic DNA from Drosophila}

For getting a good amount of genomic DNA, two male flies were pestelled in $60 \mu \mathrm{l}$ of buffer A and centrifuged for $1 \mathrm{~min}$ at 14,000 xg. The RNA containing supernatant was discarded. The pellet was washed with $400 \mu$ l buffer A, centrifuged and the supernatant was removed. The pellet was subsequently resuspended in $36 \mu$ l buffer B containing $0.36 \mu \mathrm{l}$ proteinase $\mathrm{K}(20 \mathrm{mg} / \mathrm{ml})$ and $4 \mu \mathrm{l}$ of SDS (10\%) were added. The sample was mixed properly and incubated for $1-4$ hours at $37^{\circ} \mathrm{C}$. After incubation $6 \mu$, of sodium chloride ( $\left.3 \mathrm{M}\right)$ and $20 \mu \mathrm{l}$ of phenol/chloroform were added, mixed and centrifuged for $1 \mathrm{~min}$ at 14,000 xg. $40 \mu \mathrm{l}$ of the upper phase were transferred to a fresh reaction tube and mixed with $100 \mu$ l ethanol (100\%). Centrifugation was done for $5 \mathrm{~min}$ at $14,000 \mathrm{xg}$. The supernatant was discarded and the pellet was washed with $400 \mu \mathrm{l}$ of ethanol (70\%). After repeating the centrifugation step, the supernatant was removed and the DNA sample was dried by using speed-vac. After dissolving the pellet in $20 \mu \mathrm{TE}$ buffer, the prepared genomic DNA was ready to use as template for subsequent PCR approaches.

\author{
Buffer A \\ 30 mM Tris/HCL [pH 8] \\ $100 \mathrm{mM} \mathrm{NaCl}$ \\ 19 mM EDTA \\ 0.5\% Triton X-100
}




\title{
$\underline{\text { Buffer B }}$
}

$30 \mathrm{mM}$ Tris/HCL [pH 8]

$100 \mathrm{mM} \mathrm{NaCl}$

19 mM EDTA

\subsubsection{Extraction of genomic DNA from C.elegans}

This method was performed according to Wiliams et al., 1994. To isolate genomic DNA, ten worms of each genotype were used and transferred to a PCR tube containing 50 $\mu \mathrm{l}$ of worm lysis buffer. The samples have been placed at $-80^{\circ} \mathrm{C}$ for 15 min to break down nematode bodies. The tubes were warmed up to room temperature and following incubated at $60{ }^{\circ} \mathrm{C}$ for one hour and $95{ }^{\circ} \mathrm{C}$ for $15 \mathrm{~min}$. Additional vortexing during incubation steps helped to break down worm bodies. After cooling down to $4{ }^{\circ} \mathrm{C}$ and mixing the samples were ready to use. For subsequent PCR analysis, $2.5 \mu \mathrm{l}$ were used as template.

\author{
Worm lysis buffer \\ $50 \mathrm{mM}$ KCL \\ 0.05\% Gelatin \\ $10 \mathrm{mM}$ Tris [pH 8.2] \\ $0.45 \%$ Tween 20 \\ $60 \mu \mathrm{g} / \mathrm{ml}$ Proteinase K \\ $2.5 \mathrm{mM} \mathrm{MgCl} 2$
}

\subsubsection{Polymerase chain reaction (PCR)}

PCR reactions were performed by using Taq - polymerase for general reactions or PfuS - polymerase for reactions with improved proof reading. A PCR reaction was set up with following reagents:

$\begin{array}{lll}0.5-2.5 \mu \mathrm{l} & \text { fwd Primer } & (10 \mu \mathrm{M}) \\ 0.5-2.5 \mu \mathrm{l} & \text { rev Primer } & (10 \mu \mathrm{M}) \\ 1.0 \mu \mathrm{l} & \text { dNTP mix } & (10 \mathrm{mM}) \\ 1 \mathrm{x} & \text { Buffer } & \text { (Green Taq Buffer }(10 \mathrm{x}) / \text { Phusion HF buffer }(5 \mathrm{x})) \\ 0.3 \mathrm{U} / \mu \mathrm{l} & \text { Polymerase } & \text { (Taq/PfuS) } \\ 50-200 \mathrm{ng} & \text { DNA template } \\ \text { ad } 50 \mu \mathrm{l} & \mathrm{ddH}_{2} \mathrm{O}\end{array}$


Following PCR programs were used for DNA amplification by Taq- or PfuS - polymerase:

\section{Taq / PfuS}

1. Initial Denaturation:

$95^{\circ} \mathrm{C}, 2 \mathrm{~min} / 98,5^{\circ} \mathrm{C}, 30 \mathrm{~s}$

2. Denaturation:

$95{ }^{\circ} \mathrm{C}, 30 \mathrm{~s} / 98,5^{\circ} \mathrm{C}, 5-10 \mathrm{~s}$

3. Annealing:

$\mathrm{X}{ }^{\circ} \mathrm{C}$ (Oligonucleotide specific), $30 \mathrm{~s}$

$25-35 x$

4. Extension:

$72{ }^{\circ} \mathrm{C}, 1 \mathrm{~kb} / \mathrm{min} / 72{ }^{\circ} \mathrm{C}, 1 \mathrm{~kb} / 15-30 \mathrm{~s}$

5. Final Extension:

$72{ }^{\circ} \mathrm{C}, 10 \mathrm{~min}$

\subsubsection{Site directed mutagenesis}

Site directed mutagenesis was used to establish a mutant Narf allele with an aminoacid exchange at position 388 from histidine to arginine (CAC to AGA). The PCR-reaction was performed as described before, using PfuS mix polymerase, Primer MK32/33, annealing temperature of $58{ }^{\circ} \mathrm{C}$ and an extension time of $2.5 \mathrm{~min}$. The PCR-product was subsequently digested with Dpn, a methylation dependent endonuclease, to remove the vector without mutation, which was used as template. The digested PCR-product was purified by phenol chloroform extraction (6.2.1.5) and transformed to DH5 $\alpha$ elctrocompetent cells by electroporation (pulse 4-5 ms, field strength $15.5 \mathrm{kV} / \mathrm{cm}$ ).

\subsubsection{Phenol-chloroform extraction}

A DNA/RNA sample (e.g. $50 \mu \mathrm{l}$ ) was first filled up with $\mathrm{ddH}_{2} \mathrm{O}$ to fourfold volume. Then, $200 \mu \mathrm{l}$ of Phenol/Chloroform/Isopropanol (25:24:1) was added and mixed. The Sample was centrifuged for $5 \mathrm{~min}$ at 13,000 $\mathrm{xg}$ and the upper phase was transferred to a new reaction tube. $200 \mu \mathrm{l}$ of chloroform was added, mixed well and centrifugation was repeated. The DNA was precipitated by taking the upper phase into a new reaction tube and mixing it with $1 / 10$ volume of sodium acetate (3 M) and 2.5 volumes of ethanol (100\%). The sample was kept at $-20{ }^{\circ} \mathrm{C}$ for overnight or at $-80{ }^{\circ} \mathrm{C}$ for at least $30 \mathrm{~min}$. After centrifugation at fullspeed (14.000 xg, 30min, $4^{\circ} \mathrm{C}$ ), the supernatant was removed and the pellet was washed with $200 \mu \mathrm{l}$ of ethanol (70\%). The centrifugation was repeated, the supernatant removed and the pellet was dissolved in $10-30 \mu$ of $\mathrm{ddH}_{2} \mathrm{O}$.

\subsubsection{DNA Sequencing}

DNA sequencing, used for the C.elegans studies, was performed by the company hylabs (Israel). Sequencing for the Drosophila studies was done by Eurofins Genomics (Germany). 


\subsubsection{RNA methods}

\subsubsection{RNA extraction}

RNA isolation from Drosophila thorax samples for subsequent RNAseq analysis was performed as described in the RNA isolation kit instructions.

To extract RNA from C.elegans for subsequent qPCR analysis, first the worms had to be washed in M9 buffer for several times. After the final wash, $5 \mathrm{ml}$ of DEPC treated double distilled water were added and the samples were centrifuged. The supernatant was discarded and $2 \mathrm{ml}$ of TRI-reagent per $1 \mathrm{ml}$ of worm pellet were added, gently mixed and frozen for overnight at $-80{ }^{\circ} \mathrm{C}$. The worm bodies were following broken by three cycles of crushing, thawing the samples in $42{ }^{\circ} \mathrm{C}$ and freezing them in liquid nitrogen. The samples were subsequently centrifuged for $15 \mathrm{~min}$ with $12,000 \mathrm{xg}$ at $4{ }^{\circ} \mathrm{C}$. The supernatant was transferred to a new vial and RNA was extracted as described in 6.2.1.5.

\subsubsection{RNAseq analysis}

RNA samples were isolated and handed to the Microarray and Deep-Sequencing Facility in Göttingen for subsequent analysis.

\subsubsection{Reverse transcription}

To make cDNA for subsequent qPCR analysis the following protocol was used:

1. $2 \mu \mathrm{g}$ isolated RNA

$4 \mu \mathrm{l} \quad$ RQ1 DNase $(1 \mathrm{U} / \mu \mathrm{l})$

$2 \mu \mathrm{l} \quad$ Reaction buffer (10x)

ad $20 \mu \mathrm{l} \quad$ DEPC $\mathrm{ddH}_{2} \mathrm{O}$

$\rightarrow$ Incubation for $30 \mathrm{~min}$ at $37^{\circ} \mathrm{C}$

2. $2 \mu \mathrm{l} \quad$ RQ1 DNase Stop solution

$\rightarrow$ Incubation for $10 \mathrm{~min}$ at $65^{\circ} \mathrm{C}$

3. $1 \mu \mathrm{l} \quad$ Oligo(dT) $(100 \mathrm{pmol} / \mathrm{l})$

$\rightarrow$ Incubation for $5 \mathrm{~min}$ at $70^{\circ} \mathrm{C}$

4. $10 \mu \mathrm{l} \quad$ dNTP $\operatorname{mix}(10 \mathrm{mM})$

$10 \mu \mathrm{l} \quad$ M-MLV buffer (5x)

$2 \mu \mathrm{l} \quad$ M-MLV reverse transcriptase (200 U/ $\mu \mathrm{l})$

$1.25 \mu \mathrm{l}$ Ribonucleotide inhibitor

ad $50 \mu \mathrm{l} \quad \mathrm{ddH}_{2} \mathrm{O}$

$\rightarrow$ Incubation for $60 \mathrm{~min}$ at $42^{\circ} \mathrm{C}$ 
5. The newly generated cDNA was finally stored at $-20^{\circ} \mathrm{C}$.

\subsubsection{Quantitative real-time PCR (qRT-PCR)}

All qPCR reactions were performed in triplets. For each reaction the following components were used to prepare a qPCR mastermix:

$1 \mu \mathrm{l} \quad$ cDNA (1/10 dilution)

$3.5 \mu \mathrm{l} \quad$ Primermix (fwd, rev, $1 \mu \mathrm{M}$ )

$12.5 \mu \mathrm{l} \quad$ SYBr green qPCR mix (Roche)

ad $25 \mu \mathrm{l} \quad \mathrm{ddH}_{2} \mathrm{O}$

For detecting of oxy-4, lmn-1 and pmp-3 mRNA levels, the following qPCR program was used:

1. Initial denaturation: $\quad 95^{\circ} \mathrm{C}, 3 \mathrm{~min}$

2. Denaturation: $\quad 95^{\circ} \mathrm{C}, 10 \mathrm{~s}$

3. Annealing:

4. Elongation: $\left.\begin{array}{l}60^{\circ} \mathrm{C}, 15 \mathrm{~s} \\ 72^{\circ} \mathrm{C}, 30 \mathrm{~s}\end{array}\right\} \quad 40 \mathrm{x}$

5. Melting curve: $55^{\circ} \mathrm{C}-95^{\circ} \mathrm{C}, 10 \mathrm{~s}$ (increase $0.5^{\circ} \mathrm{C}$ )

\subsubsection{Biochemical methods}

\subsubsection{Nuclei fractionation}

Staged embryos were collected, dechorionated, weighted, frozen in liquid nitrogen and stored at $-80{ }^{\circ} \mathrm{C}$ until the fractionation experiment was performed. To minimize protein degradation during fractionation, all steps were performed on ice or at $4{ }^{\circ} \mathrm{C}$ with precooled buffers. Embryos were suspended in Hepes buffer in a ratio of $150 \mathrm{mg}$ of embryos to $1 \mathrm{ml}$ of buffer. Embryos were crushed by douncing 6 times with a potter and $20 \mu \mathrm{l}$ were saved for western blot analysis and named as "total”. To pellet nuclei and other cell components, the sample was centrifuged for $5 \mathrm{~min}$ at $1.000 \mathrm{xg}$. The supernatant was removed and centrifuged with high speed (14,000 xg) for $10 \mathrm{~min}$ and a "cytoplasm” fraction was saved. Nuclei were subsequent fractionated by using a sucrose gradient. The pellet was resuspended in $1 \mathrm{ml}$ Hepes buffer with $0.25 \mathrm{M}$ sucrose and layered over a $5 \mathrm{ml}$ cushion of Hepes buffer with $0.35 \mathrm{M}$ sucrose. Centrifugation was performed at 1,400 $\mathrm{xg}$ in a swing out rotor for $5 \mathrm{~min}$. After discarding supernatant the nuclei pellet was resuspended in $1 \mathrm{ml}$ Hepes buffer with $0.35 \mathrm{M}$ sucrose and layered over a $5 \mathrm{ml}$ cushion of Hepes buffer with $0.8 \mathrm{M}$ 
sucrose. The centrifugation step was repeated with 2,000 xg for $5 \mathrm{~min}$. To enhance the nuclei separation, the last sucrose step was done twice. After nuclei isolation, the pellet was washed two times in $1 \mathrm{ml}$ Hepes buffer. Samples were saved before and after centrifugation (4,000 xg, $5 \mathrm{~min}$ ) and named as “isolated nuclei” and “washing step”. Kugelkern solubilization was done by incubating the nuclei pellet for at least $15 \mathrm{~min}$ on a wheel in $1 \mathrm{ml}$ Hepes buffer with 1\% TritonX-100 and $150 \mathrm{mM}$ sodium chloride. The sample was centrifuged for 15 min with $14,000 \mathrm{xg}$. A volume amount representing 4,500 embryos was saved as “input” sample. The remaining sample was used for subsequent immunoprecipitation experiments. This method was modified according to established protocols of the Lamond laboratory (online available at http://www.trinklelab.com/pubpdf/CellFractionation.pdf).

\author{
$\underline{\text { Hepes Buffer }}$ \\ 15 mM Hepes \\ $5 \mathrm{mM} \mathrm{MgCl}_{2}$ \\ $10 \mathrm{mM} \mathrm{KCL}$ \\ $[\mathrm{pH}$ 7.4] (KOH)
}

Sucrose was added in a final concentration of $0.25 \mathrm{M}, 0.35 \mathrm{M}$ or $0.8 \mathrm{M}$.

To all Hepes buffers, 1x protease inhibitor cocktail, $1 \mathrm{mM}$ DTT and $0.1 \mathrm{mM}$ PMSF were added freshly.

\title{
6.2.3.2 GFP-protein pulldown using GFP dynabeads
}

To analyze biochemical interaction partners of Kugelkern, GFP-Kuk expressing embryos were collected and nuclei fractionation was performed as described in 6.2.3.1. After taking an "input" sample for western blot analysis, the rest of the fraction sample was incubated with $50 \mathrm{nM}$ of biotinylated GFP binding protein (gift from Prof. Görlich) for one hour on ice. $20 \mu$ l of Magnetic Streptavidin T1 dynabeads, resuspended by gentle vortexing, were taken for binding assay. The beads were washed trice in $1 \mathrm{x}$ PBS containing $0.1 \%$ BSA and concentrated by using a magnetic field. Proteinlysate with bound GFP binder was transferred to the beads and incubated for one hour on the wheel. After incubation, an “unbound” sample was taken and beads were washed trice with Hepes buffer (6.2.3.1). To minimize unspecific binding, the GFP binder was cleaved off from beads via an intrinsic cleavage site. Therefore, $100 \mu \mathrm{M}$ of Sumostar Protease (gift from Prof. Görlich, MPI for Biophysical Chemistry, Göttingen), diluted in 1x PBS, were added to the dynabeads and 
incubated again for one hour at $4^{\circ} \mathrm{C}$ on a wheel. Afterwards, samples of the supernatant, representing the "cleaved” fraction and the "beads”, were analyzed by western blot.

\subsubsection{Protein precipitation}

Fractionation samples that exceed a volume of $20 \mu \mathrm{l}$ were precipitated by adding TCA in a final concentration of $15 \%$. Samples were incubated first at $-20{ }^{\circ} \mathrm{C}$ and second on ice for $15 \mathrm{~min}$. Next, a centrifugation was performed at $14,000 \mathrm{xg}$ for $10 \mathrm{~min}$. The supernatant was removed and the protein pellet was washed twice with ice-cold aceton, followed by a centrifugation step. The pellet was finally resuspended in an appropriate volume of 2x sample buffer. In the case that sample buffer color turned to yellow, $\mathrm{pH}$ was adjusted by adding a drop of $1 \mathrm{M}$ Tris $\mathrm{pH}>8$. The samples were boiled at $95^{\circ} \mathrm{C}$ for $3-5 \mathrm{~min}$ and used for SDS-page.

\subsubsection{Western Blot}

\subsection{Sample preparation}

To analyze different samples via western blot it is essential to load an appropriate amount of protein. Following protocols were used in this study.

Embryos and larvae: 15 embryos or larvae were collected in $10 \mu \mathrm{l}$ of water, freezed in liquid nitrogen and macerated. Afterwards $10 \mu \mathrm{l}$ of 2x sample buffer were added. Samples were vortexed, boiled for $3 \mathrm{~min}$ at $95^{\circ} \mathrm{C}$ and centrifuged for 1 - $2 \mathrm{~min}$ at $14.000 \mathrm{xg}$. For following analysis the lysate of 10 embryos and L1 larvae, 1.5 L2 larvae and 0.5 L3 larvae were used. Fly extract: 10 male flies were collected and macerated in $50 \mu$ l water. One volume of 2x sample buffer was added. The samples were vortexed, boiled for $3 \mathrm{~min}$ at $95{ }^{\circ} \mathrm{C}$ and centrifuged for $10 \mathrm{~min}$ at $14.000 \mathrm{xg}$. The lysate of one fly was used for subsequent analysis. Thoraces: 10 thoraces were prepared (compare 6.2.5.5) and macerated in $50 \mu \mathrm{l} 1 \mathrm{x}$ sample buffer and sample volume was filled up to $70 \mu \mathrm{l}$ of $1 \mathrm{x}$ sample buffer. The samples were vortexed, boiled for $3 \mathrm{~min}$ at $95{ }^{\circ} \mathrm{C}$ and centrifuged for $2-3 \mathrm{~min}$ at $14.000 \mathrm{xg}$. The lysate of of 1.5 - 2.5 thoraces was used for subsequent analysis.

Worm extract: 25 worms were collected in a reaction tube and washed several times with M9 buffer. The supernatant was completely removed. The worms were frozen in liquid nitrogen and macerated after adding $25 \mu \mathrm{l}$ of $1 \mathrm{x}$ sample buffer. The sample was boiled for $3 \mathrm{~min}$ at $95^{\circ} \mathrm{C}$ and centrifuged for $2-3 \mathrm{~min}$ at $14.000 \mathrm{xg}$. The lysate of 10 worms was used for western blot analysis. 


\subsection{Western blot}

The samples were prepared as described before and loaded on a 10\% SDS-gel, unless otherwise stated. The SDS-gel electrophoresis was performed at $17 \mathrm{~mA}$. Proteins were following blotted to a nitrocellulose membrane for 1.5 hours at $60 \mathrm{~mA}$ by using a semi-dry transfer. Unspecific antibody binding was minimized by blocking with 5\% milk/1x PBS for 1 hour at room temperature. Primary antibodies were diluted in $1 \%$ milk/1x PBS with $0.1 \%$ Tween20 and incubated for one hour at room temperature or overnight at $4{ }^{\circ} \mathrm{C}$. Membrane was first rinsed and then washed three times for five minutes in 1x PBS/0.1\% Tween20. Incubation and washing steps were repeated for the secondary antibody. Bound secondary antibodies coupled with fluorescence were finally detected using Odyssey CLx Imaging system.

\subsubsection{Antibodygeneration}

\subsubsection{Expression of recombinant proteins}

Plasmid DNA coding for the protein was transformed into the E.coli strain BL21DE (rosetta). A $100 \mathrm{ml} \mathrm{LB}{ }^{\text {Amp }}$ culture was set up and incubated at $37^{\circ} \mathrm{C}$ for overnight. To express recombinant proteins in a large scale, $1 \mathrm{l} \mathrm{LB}^{\mathrm{Amp}}$ culture was inocculated with the overnight culture to an optical density (OD) of 0.1 . The culture was afterwards incubated at $37^{\circ} \mathrm{C}$ until it reached an OD-value of 0.5. A $1 \mathrm{ml}$ sample for subsequent SDS-page was taken, centrifuged for $1 \mathrm{~min}$ at 14,000 $\mathrm{xg}$ and dissolved in 1x sample buffer. Protein expression was then induced by adding $1 \mathrm{mM}$ of IPTG to the culture and incubating it for 1 - 4 hours at $18{ }^{\circ} \mathrm{C}$. In between, further samples were taken. Cell collection was performed by centrifugation at room temperature for $20 \mathrm{~min}$ at 4,800 xg. Cell lysis was improved by a preceding overnight freezing step at $-20^{\circ} \mathrm{C}$. Cells were afterwards thawed on ice and $10 \mathrm{ml}$ lysis buffer per $1 \mathrm{~g}$ pellet were added and thoroughly suspended. A sample was taken, centrifuged and sample buffer was added to the supernatant (soluble fraction) and the pellet (insoluble fraction). Then, protease inhibitor PMSF (final concentration 1mM), Lysozym (final: $1 \mathrm{mg} / \mathrm{ml}$ ) and a spatula tip of DNase were added. The cell suspension was mixed and incubated for $30 \mathrm{~min}$ on ice. A sample of the soluble and insoluble fraction was taken. Next, cells were disrupted by sonification (pulse: 50 , power 5 , cycles of one minute sonification and two minutes pause were repeated three times) or using the Microfluidizer and samples were taken. For soluble proteins, the supernatant was transferred to a new vial, centrifuged 
for $10 \mathrm{~min}$ at $14.000 \mathrm{xg}$ and $4{ }^{\circ} \mathrm{C}$ and purified as described in 6.2.4.2. Insoluble proteins, present in the pellet, were purified under denatured conditions (see 6.2.4.3).

\author{
Lysis buffer: \\ 20 mM Na-phosphate, pH 8.0 \\ $500 \mathrm{mM} \mathrm{NaCl}$ \\ 20 mM Imidazole
}

\title{
6.2.4.2 Native protein purification via His-tag
}

Before protein purification a Ni-His-Trap column was prepared and after purification regenerated as described in manufacturer`s instruction. Protein sample was loaded in a constant flow rate of $0.5-1 \mathrm{ml} / \mathrm{min}$ using the Äkta-system. The flow through was collected. The column was washed with washing buffer using more than 10 column volumes (CV). Elution was achieved by establishing an imidazole gradient between wash- and elution buffer. Elution fractions were collected every 0.5 CV. Samples of all purifications steps were taken and analyzed in a SDS-Page. Elution fractions, containing the desired protein, were pooled and a buffer exchange was performed using PD10 columns following manual instructions. To finally store the purified protein $50 \%$ of glycerol was added and the sample was kept at $-80^{\circ} \mathrm{C}$.

\author{
Wash buffer: \\ 20 mM Na-phosphate, pH 8.0 \\ $500 \mathrm{mM} \mathrm{NaCl}$ \\ 40 mM Imidazole \\ Elution buffer: \\ $20 \mathrm{mM}$ Na-phosphate, [pH 8.0] \\ $500 \mathrm{mM} \mathrm{NaCl}$ \\ $500 \mathrm{mM}$ Imidazole
}

\subsubsection{Denatured protein purification via His-tag}

Insoluble proteins, present in the pellet, were first suspended in $25 \mathrm{ml}$ of lysis buffer and mixed for one hour at room temperature. The suspension was centrifuged for $10 \mathrm{~min}$ at $10,000 \mathrm{xg}$ and the solubilized pellet extract was added to $3 \mathrm{ml}$ of Ni-sepharose beads, which 
were equilibrated with lysis buffer before. Binding was allowed under rotation at room temperature for 30 - 60 min. Next, the beads suspension was loaded onto a drop column. The flow-through was collected and the beads were washed three times with $6 \mathrm{ml}$ washing buffer. Proteins were eluted in $151 \mathrm{ml}$ fractions of elution buffer. Eluates were analyzed by SDS-Page and the identified protein containing fractions were kept at $4{ }^{\circ} \mathrm{C}$ for further usage. The buffers were prepared according to “The QIAexpressionist TM”, 5 ${ }^{\text {th }}$ edition 2003.

Lysis buffer
$100 \mathrm{mM} \mathrm{NaH}_{2} \mathrm{PO}_{4}$
$10 \mathrm{mM} \mathrm{TrisCl}$
$6 \mathrm{M} \mathrm{GuHCl}$
[pH 8.0], $\mathrm{NaOH}$
Wash buffer
$100 \mathrm{mM} \mathrm{NaH}{ }_{2} \mathrm{PO}_{4}$
$10 \mathrm{mM}$ TrisCl
$8 \mathrm{M} \mathrm{Urea}$
[pH 6.3], $\mathrm{HCl}$
Elution buffer
$100 \mathrm{mM} \mathrm{NaH}{ }_{2} \mathrm{PO}_{4}$
$10 \mathrm{mM}$ TrisCl
$8 \mathrm{M} \mathrm{Urea}$
[pH 4.5], $\mathrm{HCl}$

\subsubsection{Immunisation}

Polyclonal antibodies were produced by either Charles River (Wilmington, USA) or BioScience (Göttingen, Germany). Received final sera were tested in western-blot analysis as well in immunostainings and if necessary further purified.

\subsubsection{Antibody affinity purification}

Antibody affinity purification is a method based on the specific interaction of an antibody and it`s antigen. To specifically isolate the desired antibody it is necessary to couple 
the antigen against $\mathrm{CNBr}$-activated Sepharose 4B. This method was performed according to manufacturer`s instruction.

\title{
Protein preparation
}

As denatured purified proteins were kept in $8 \mathrm{M}$ urea, which would react with the BrCN groups, it was necessary to remove the urea. Therefore, a dialysis sleeve was prepared as mentioned in manual`s description and a dialysis against coupling buffer (three times 100 volumes) was performed.

\author{
Coupling buffer \\ $100 \mathrm{mM} \mathrm{NaHCO} 3$ \\ $300 \mathrm{mM} \mathrm{NaCl}$ \\ $1 \%$ SDS \\ [pH 8.3], $\mathrm{NaOH}$
}

\section{Preparation of CnBr-activated Sepharose 4B}

$1 \mathrm{~g}$ of dry Sepharose 4B was swelled in $10 \mathrm{ml}$ of $1 \mathrm{mM} \mathrm{HCl}$ for 15 minutes. The swollen beads (gel) were washed on a sintered glass filter with $200 \mathrm{ml}$ of $1 \mathrm{mM} \mathrm{HCl}$.

\section{Coupling of antigen and beads}

The gel was equilibrated in $5 \mathrm{ml}$ coupling buffer per $1 \mathrm{~g}$ of dry beads and immediately afterwards the protein suspension was added in a 2:1 buffer to gel ratio. Beads suspension was mixed for 3 hours at room temperature or at $4{ }^{\circ} \mathrm{C}$ for overnight. The beads were allowed to settle down, supernatant was removed and tested for its protein concentration ( $\left.\mathrm{A}_{280}\right)$ to confirm efficient binding. To minimize unspecific binding, the beads were blocked for 2 hours at room temperature in blocking buffer. The suspended beads were poured into a column where they were washed for five times each with washing buffer I, II and 1x PBS.

\section{Blocking buffer}

$0.1 \mathrm{M}$ Tris/HCl

[pH 8.0] 
Wash buffer I

$0.1 \mathrm{M}$ Na acetate

$0.5 \mathrm{M} \mathrm{NaCl}$

[pH 4.0]

Wash buffer II

$0.1 \mathrm{M}$ Tris/HCl

$0.5 \mathrm{M} \mathrm{NaCl}$

[pH 8.0]

\section{Antibody affinity chromatography}

$5-10 \mathrm{ml}$ of the final serum were centrifuged at $14,000 \mathrm{xg}$ for $30 \mathrm{~min}$ at $4{ }^{\circ} \mathrm{C}$ and the cleared serum was applied to the prepared affinity column. The flow through was collected and loaded again up to seven times. The column was washed with $10 \mathrm{CV}$ of $1 \mathrm{x}$ PBS followed by $10 \mathrm{CV}$ of $1 \mathrm{x}$ PBS $+300 \mathrm{mM} \mathrm{NaCl}$. Elution buffer was added and elution fractions of $900 \mu \mathrm{l}$ were collected in reaction tubes containing $100 \mu \mathrm{l}$ neutralization buffer. The protein concentration of the elution fractions was measured and protein containing samples were pooled. The antibody was finally concentrated to $1 \mathrm{mg} / \mathrm{ml}$ using Viva spin columns. 0.02\% of Na-azide was added and the antibody was stored at $4{ }^{\circ} \mathrm{C}$.

Neutralisation buffer:

$1 \mathrm{M}$ Tris/HCl

$[\mathrm{pH}>9.0]$

Elution buffer:

$50 \mathrm{mM}$ Glycine

[pH 2.5]

\section{Affinity column regeneration}

The used column was washed with $10 \mathrm{CV}$ of washing buffer I, II and 1x PBS and finally stored in $1 \mathrm{x}$ PBS $+0.01 \%$ Sodium azide at $4{ }^{\circ} \mathrm{C}$. 


\subsubsection{Generation of a nuclear pore complex (NPC) specific antibody}

To generate a Nup153 specific antibody, 352 amino acids of the PA-isoform were used. The corresponding DNA sequence was cloned into pGEX-60H vector by M. Clever. The recombinant protein was expressed for 4 hours at $18{ }^{\circ} \mathrm{C}$ and purification was performed under native conditions using His-tag. Immunisation was done by Charles River into guinea pig and rabbit. The Nup153 (rabbit) antibody was affinity purified and concentrated to $1 \mathrm{mg} / \mathrm{ml}$. Immunofluorescence and western blot analysis confirmed that the anti-Nup153 antibody can be used as a marker for nuclear pore complexes. However, it is likely that the antibody also recognizes other proteins of the NPCs as the aminoacid sequence contains several phenylalanine-glycine repeats (FG-repeats), which are evolutionary conserved within nucleoporins (Figure 48).

VMNKSSDEECIACQTPNSQARNSNSESALISSISSSSASFSGSLSRPSSRSSSGSTSTC GSVCSGSIVSISSTTESAKALSAKKVPPKPDAGFQQLVAAQKTSTWECEACLAKND MSRKTCICCEQMMPEAFNPAATTANSAASSVPKFRFGFSHVKEVVKPSVETTTTPA PTSAQFSFGFGQSNQGKDVADSKKTEAPKTFMFGVSKVEEPKTVSFGTGIKETTAT SSTEATAPTPAAAAPAPVQFVFKAPTTATTASSLTTTISTTSNAPALGGFSFGAPSSS STVSSSTTSTSANPAAVKPMFSWSGAGSAVSSTSSSQQPVAKAPTLGFGVSSSTVT TTTTSTKVFAF

Figure 48: Amino acid sequence used for Nup153 antibody

Green colored amino acids show evolutionary conserved FG-repeats.

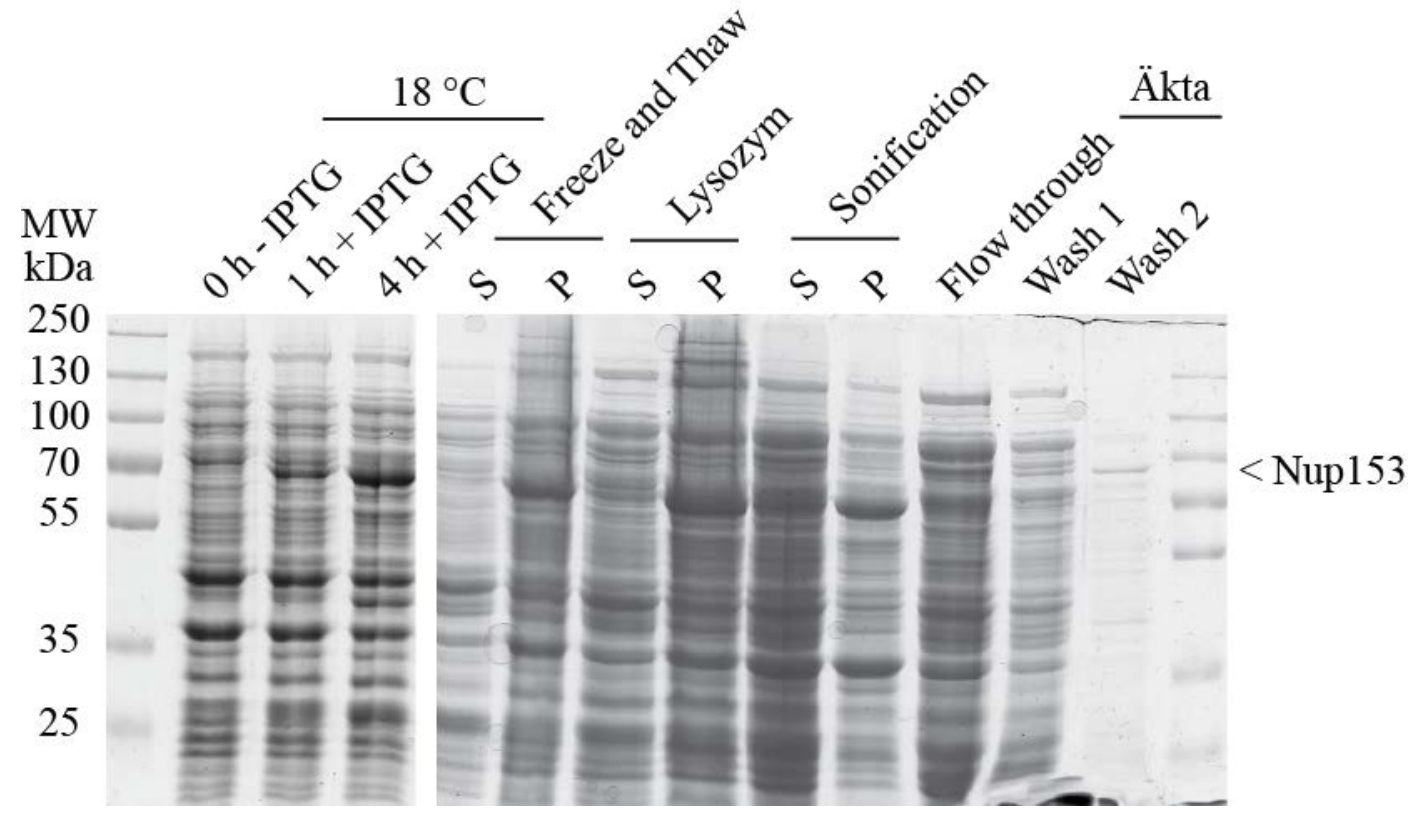




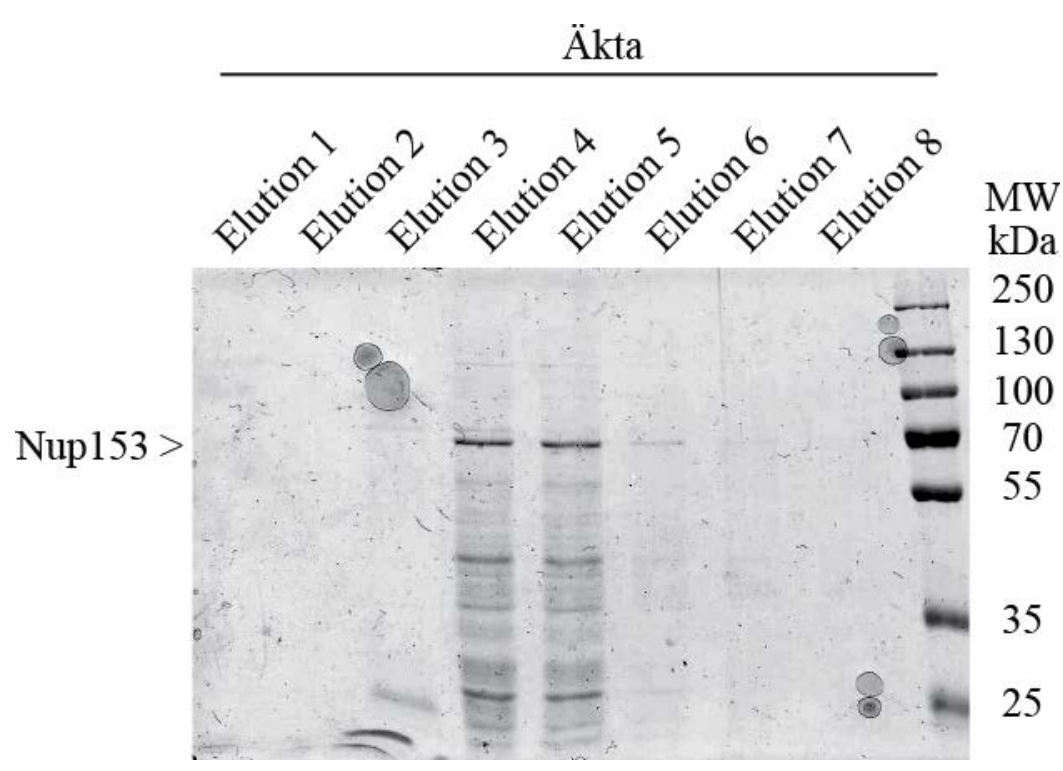

Figure 49: Expression and purification of the pGEX-60H-Nup153 protein

Nup153 was expressed for 4 hours at $18^{\circ} \mathrm{C}$. Preparation of cell lysates was performed by freezing and thawing the sample followed by lysozyme treatment and sonification. The success of cell disruption was proved by loading a sample of the soluble fraction ( $\mathrm{S}$ - supernatent) and insoluble fraction (P - pellet) of each step. Purification was done under native conditions via His-Tag. Elution was established by using an imidazole gradient. The protein was eluted between $240 \mathrm{mM}$ and $395 \mathrm{mM}$ of imidazole (elution fraction 4 and 5). Sample analysis was performed using a 10\% SDS-gele.

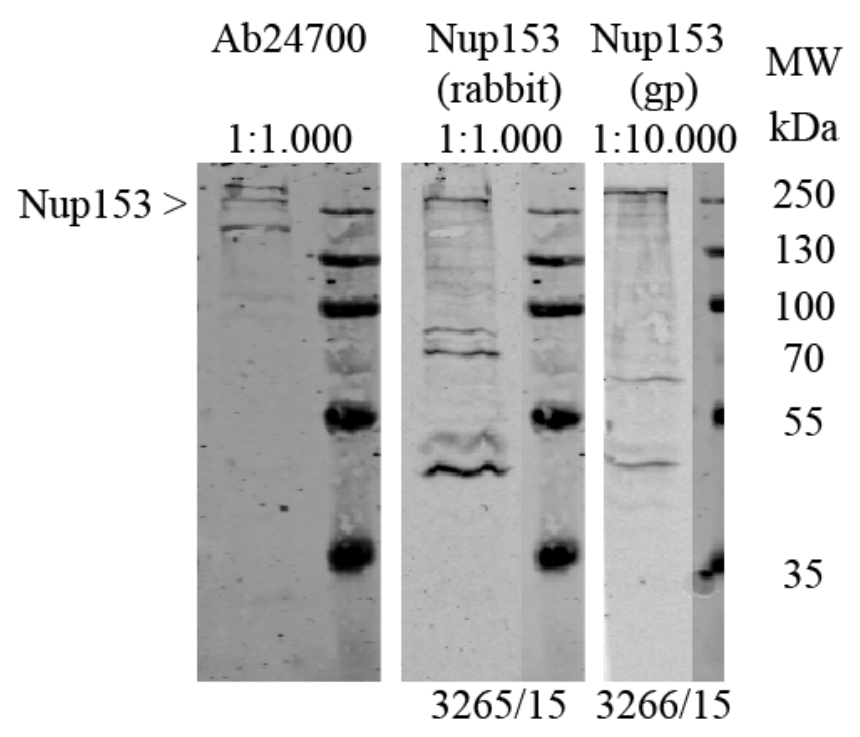

Figure 50: Specificity of the generated Nup153 antibody

The western blot analysis shows that the antibodies bind to Nup153 protein. A commercial available antibody (Ab24700) which stains for Nup153, Nup214 and p62 was used as comparism. 


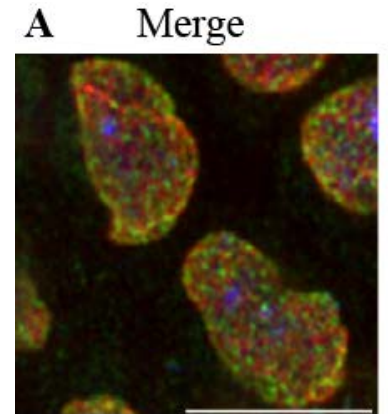

B Merge

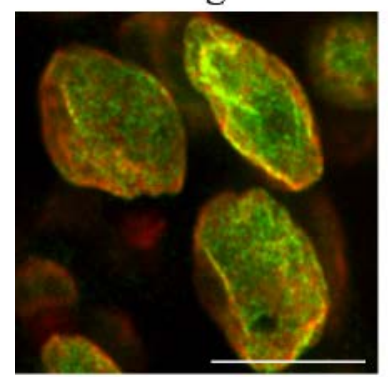

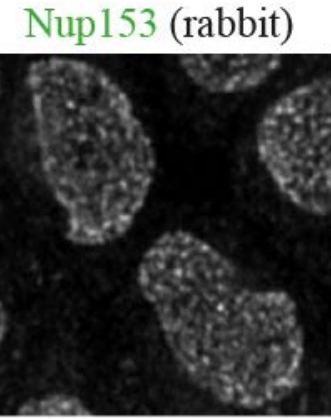

Nup153 (gp)

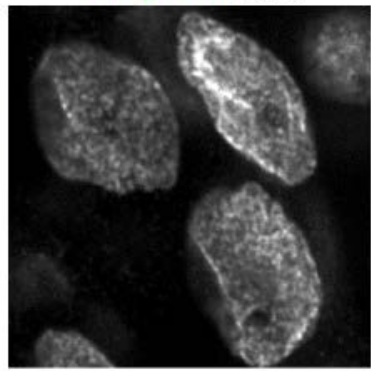

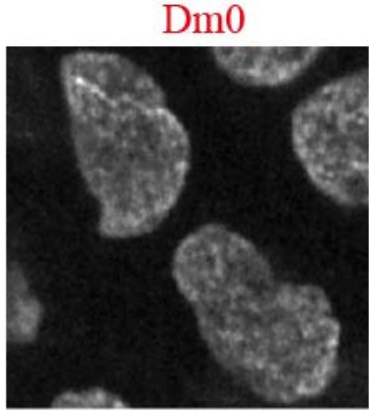

$\mathrm{Dm} 0$

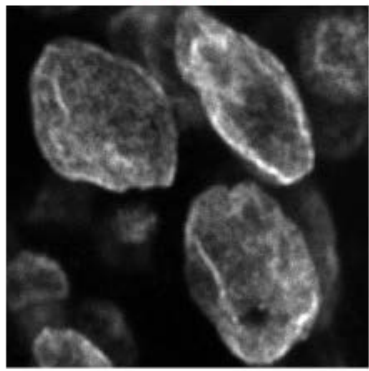

Scale Bar: $10 \mu \mathrm{m}$

Figure 51: Nup153 antibody stains specific nuclear pore complexes

Immunofluorescence analysis of amnioserosa cells with antibodies against Dm0 as a marker for the nuclear envelope and Nup153 A) generated in rabbit and B) in guinea pig.

\subsubsection{Staining methods}

\subsubsection{Embryo fixation}

Embryos of desired stages were dechorionized on apple-juice plates by treating them with $50 \%$ of Klorix for $90 \mathrm{~s}$. The embryos were collected in a net and washed several times with water to remove the Klorix completely. Afterwards, embryos were transferred to a scintillation vial containing $5 \mathrm{ml}$ heptane, $4.5 \mathrm{ml} \mathrm{1x}$ PBS and $0.5 \mathrm{ml}$ formaldehyde (37\%) and fixed for $20 \mathrm{~min}$ at room temperature under constant shaking. The lower phase was removed, $5 \mathrm{ml}$ of methanol were added and embryos were vortexed for $30 \mathrm{~s}$ to remove the vitellin membrane. Devitellinized embryos were collected and transferred to a reaction tube. The embryos were washed with methanol for several times and finally stored at $-20^{\circ} \mathrm{C}$.

\subsubsection{Embryo immunostaining}

In methanol stored embryos were rinsed several times and washed once for $5 \mathrm{~min}$ with $1 \mathrm{ml}$ 1x PBS/0.1\% Tween (PBT). Blocking was performed by incubation of 1 hour in $500 \mu \mathrm{PBT}$ containing 5\% BSA at room temperature. All incubations were done under constant rotation. Primary antibodies were diluted in the indicated ratio in $500 \mu \mathrm{l}$ of PBT 
with $1 \%$ BSA and incubated for 2 hours at room temperature or overnight at $4{ }^{\circ} \mathrm{C}$. The embryos were rinsed three times and washed for four times for each 15 minutes with $500 \mu \mathrm{l}$ PBT. Secondary antibody incubation and following washing steps were performed using the same procedure. Embryos were stained with DAPI (1:250, $0.2 \mathrm{mg} / \mathrm{ml})$ for 7 - 10 minutes, rinsed three times and washed once for 5 minutes with PBT. Mounting was done by using Aquapolymount medium.

\subsubsection{Imaginal discs preparation and fixation}

To prepare imaginal discs, L3 larvae were dissected in ice-cold 1x PBS by holding them with forceps at the anterior end and tearing two thirds of the larvae away. Mouth hooks with attached and colorless appearing imaginal discs were pulled out. The tissue was collected in staining tubes containing 1x PBS. Fixation was performed by incubating samples in $4 \%$ paraformaldehyde in 1x PBS for 40 minutes at room temperature under constant shaking.

\subsubsection{Imaginal discs immunostaining}

The fixated samples were washed once in 1x PBS/0.3\% Triton X-100 for 20 minutes and blocked with 5\% BSA in $1 \mathrm{x}$ PBS/0.3\% Triton X-100 for one hour at room temperature. All incubation steps were carried out under constant horizontal shaking. Primary antibodies were diluted in the indicated ratio in $500 \mu \mathrm{l}$ of $1 \mathrm{x}$ PBS/1\% BSA/0.1\% Tween and incubated overnight at $4{ }^{\circ} \mathrm{C}$. The samples were washed three times with $1 \mathrm{x}$ PBS/0.3\% Triton X-100 for 20 minutes each. Secondary antibody incubation and following washing steps were performed as for the first antibody. Samples were stained with DAPI $(1: 250,0.2 \mathrm{mg} / \mathrm{ml})$ for 7 - 10 minutes, rinsed three times and washed twice for 10 minutes with 1x PBS/0.3\% Triton X-100. Finally, larvae mouth hooks with attached imaginal discs were transferred to a drop of 1x PBS on a glass slide and excess tissue was removed with forceps. Aquapolymount medium was added to the isolated imaginal discs and samples were mounted with a cover slide and dried over night at $4{ }^{\circ} \mathrm{C}$.

\subsubsection{Thorax muscle fixation and immunostaining}

For every staining, 7 - 10 thoraces were prepared by removing head, legs and abdomen from anesthetized flies. The samples were collected in a reaction tube on ice. Fixation was achieved by incubating thoraces with 4\% formaldehyde in 1 x PBS/0.3\% Triton $\mathrm{X}-100$ for 20 minutes at room temperature under constant shaking. Samples were washed two times with $1 \mathrm{x}$ PBS for 5 minutes each and transferred to sticky tape with the ventral side 
up. Muscle tissue was deposit by cutting thoraces midsagittal. The thorax halves were transferred back to a $500 \mu \mathrm{l}$ reaction tube and blocked with 5\% BSA in 1x PBS/0.3\% Triton $\mathrm{X}-100$ for one hour at room temperature. Antibody stainings were performed as described in 6.2.5.2. This method was modified according to Weitkunat et al., 2014.

\subsubsection{Phalloidin staining of worms}

Worms were collected at the desired developmental stages and washed with M9-Buffer. Permeabilisation took place by freezing worms in liquid nitrogen. The samples were thawed at room temperature and consecutively fixed by methanol and acetone incubation for $5 \mathrm{~min}$ at $-20^{\circ} \mathrm{C}$ each. After incubating worms in blocking solution for $20 \mathrm{~min}$, the samples were stained with flurescent phalloidin for one hour at room temperature. The samples were washed trice with 1x PBS containing 3\% BSA and mounted in 2\% of n-propyl galate in glycerol on agarose slides. This method was performed according to established protocols of the Gruenbaum laboratory.

\section{$\underline{\text { M9 Buffer }}$}

$\begin{array}{ll}\mathrm{KH}_{2} \mathrm{PO}_{4} & 22 \mathrm{mM} \\ \mathrm{Na}_{2} \mathrm{HPO}_{4} & 42 \mathrm{mM} \\ \mathrm{NaCl} & 86 \mathrm{mM}\end{array}$

\section{Blocking solution}

$10 \%$ milk

3\% bovine serum albumin

$0.1 \%$ Tween20 (v/v)

in 1x PBS

\subsubsection{Immunostaining of worms}

Cleaned slides had to be coated first with $50-100 \mu \mathrm{l}$ of poly Lysine solution $(0.1 \% \mathrm{v} / \mathrm{v})$. After $30 \mathrm{~min}$ of incubation, excess solution was removed and slides were let to dry completely. Around 20 worms of the desired stage were transferred by a platinum wire to a drop of $10 \mu \mathrm{l}$ of M9 buffer which was placed on the poly lysine area. Afterwards a 24 x $50 \mathrm{~mm}$ cover slip was placed perpendicular to the slide. To break nematodes tissue, gentle pressure was given at the slide edges. The samples were following frozen by liquid nitrogen and the cover slip was removed afterwards. Fixation was done successively by 
methanol and acetone incubation each for $15 \mathrm{~min}$ at $-20^{\circ} \mathrm{C}$. Remaining's of fixation solution was removed by a washing step with 1x PBS/0.01\% Tween20 (PBS-T) for $10 \mathrm{~min}$ at room temperature. The worm samples were then blocked in 1x PBS/0.1\% milk solution for 15 min at room temperature. Antibody staining was performed by pipetting $50 \mu \mathrm{l}$ of the diluted antibody to the sample, covering it with parafilm and incubating at $4{ }^{\circ} \mathrm{C}$ for overnight in a humidity chamber. The glass slides were then quickly washed in 1x PBS to carefully remove the parafilm. Three washing steps in 1x PBS each for $20 \mathrm{~min}$ followed. Then, $50 \mu \mathrm{l}$ of the diluted secondary antibody were pipetted to the samples. The samples were covered with parafilm and incubation took place for 3 hours at room temperature in a humidity chamber. Washing steps were repeated and DAPI $(1: 1.000,1 \mathrm{mg} / \mathrm{ml})$ staining was performed by pipetting $100 \mu \mathrm{l}$ of to the samples and incubating it for $10 \mathrm{~min}$ at room temperature. The sample were finally incubated for at least 10 min in 1x PBS and mounted in a drop of 2\% n-propyl galate in glycerol on agarose slides. This method was performed according to established protocols of the Gruenbaum laboratory.

\subsubsection{Fly work methods}

\subsubsection{Wing preparation}

To study gain-of-function and loss-of-function effects on wing development, wings were removed from the adult fly body and mounted into a medium consisting of Hoyers medium and lactate acid in a 1:1 ratio. The samples were incubated overnight at a temperature of $50{ }^{\circ} \mathrm{C}$ and analyzed.

\subsubsection{Lifespan}

To analyze the lifespan of a distinct fly population newly hatched male flies were collected every 2 - 3 days. For each genotype, three to four replicates with each $\sim 75$ males were collected under short anesthesia and transferred to a fly cage with a filter. Cages were closed with plates containing fly food. Induction with the GeneSwitch system was carried out by adding additional mifepriston (RU-486) with concentrations ranging from 2 to $200 \mu \mathrm{M}$ directly to fly food. Fly cages were kept at $25{ }^{\circ} \mathrm{C}$ with a humidity of $70 \%$ and a day/night cycle of 12 hours. New fly food was provided every 2 - 3 days and death events were counted at the same time. Escaped flies were noted as censored. Survival data were analyzed by using OASIS (Yang et al., 2011) an online application tool for survival analysis 
(log-rank test) and data were illustrated by using GraphPad Prism6. Lifespan assays were performed according to Brandt et al., 2008.

\subsubsection{Negative geotaxis}

To analyze climbing behavior over time newly hatched male flies ( 0 - 3 days) were collected and kept in cage at $25^{\circ} \mathrm{C}$ with a humidity of $70 \%$ and a day/night cycle of 12 hours during experimental analysis. The climbing assay was performed once a week. Therefore, 25 males were shortly anesthetized and transferred individually to vertical climbing tubes. After flies were completely awake, males were tapped to the bottom and immediately $10 \mathrm{~s}$ test time started. Climbing high was noted and measurements were repeated 3 - 4 times for each fly. Mean values were determined and the climbing high of control flies in the first week was set as $100 \%$ negative geotaxis. This method was performed according to Gargano et al., 2005.

\subsubsection{Survival rate assay}

Flies were kept at $25{ }^{\circ} \mathrm{C}$. 100 embryos of each genotype were collected without dechorionization and transferred to fresh apple-juice plates coated with yeast. To distinguish between homozygous and heterozygous genotypes an embryo sorting, based on GFP expression, was performed if necessary. Hatched L1 larvae were counted and moved to fly food vials where following pupae and hatched adults were counted as well. Finally, percentage of the survival rate during development was analyzed.

\subsubsection{Microinjection of Drosophila embryos}

Early w1118 embryos were collected, dechorionated, arranged in line and transferred to a cover slide with n-Heptane. The embryos were incubated for $7 \mathrm{~min}$ in a desiccation chamber and subsequently covered with $10 \mathrm{~S}$ VoltaLef oil. An injection mix, consisting of the desired DNA and a helper plasmid with a 3:1 ratio, was prepared and injected into the posterior pole of the embryos with a final concentration of $0,3-0,8 \mu \mathrm{g} / \mu \mathrm{l}$. Injected embryos were transferred to a humidity chamber and kept at $18^{\circ} \mathrm{C}$. First larvae were collected, transferred to normal fly food vials and G0 - flies were separately crossed to w1118 flies. Offsprings were scored for red eyes and individual white ${ }^{+}$flies were used to establish transgenic fly lines.

For site specific integration, a vector, containing the desired DNA and an attP site, were injected in a final concentration of $0.1 \mu \mathrm{g} / \mathrm{ul}$ to embryos containing a genomic attB-site. 


\subsubsection{Worm work methods}

Worm work methods were performed according to Dobrzynska et al., 2016, unless otherwise stated.

\subsubsection{Synchronizing worms}

Worms carrying embryos were collected by washing NGM plates with M9-buffer. Animals were washed 2-3 times by spinning them down (2.500 xg) and adding new M9 buffer. The supernatent was removed and replaced by bleach solution. The worms were opened by vortexing for around $4 \mathrm{~min}$. In order to remove the bleach solution, worms were washed up to 5 times with M9 buffer. Embryos were spinned down (16.000 xg) and collected in $20-50 \mu \mathrm{l}$ of M9-buffer. About $10 \mu \mathrm{l}$ were subsequently seeded per NGM or feeding plate. If it was necessary to synchronize worms to L1 stage, eggs were transferred into a 4 $\mathrm{ml}$ M9-buffer containing $15 \mathrm{ml}$ falcon and incubated over night at $20^{\circ} \mathrm{C}$. L1 worms were collected and transferred to plates.

\section{Bleach solution}

2 Volumes Water

1 Volume Sodium Hypochlorite Solution (15\%)

\subsubsection{Oxygen sensitivity assay}

Worm eggs were isolated and cultured on OP50 seeded NGM plates and incubated for 5 days in the presence of $75 \%$ of oxygen at $20^{\circ} \mathrm{C}$. Control worms were grown under normoxic conditions. Afterwards the developmental stages of the hatched worms were determined. This method was performed according to Fujii et al., 2009.

\subsubsection{Time course analysis}

To analyze worm development under distinct conditions, nematodes were first synchronized to L1 stage (6.2.7.1), transferred to either NGM or RNAi plates and grown for three days at $20^{\circ} \mathrm{C}$. At different time points, worms were taken for microscopic analysis. To prevent strong nematode movement, worms were mounted in levamisol (2 mM). Worm length was measured by using ImageJ and the data analysis was performed with GraphPad Prism6. 


\subsubsection{Pumping experiment}

For studying pumping rates, worm eggs were isolated and grown on OP50 seeded NGM plates at $20^{\circ} \mathrm{C}$ until they reached adulthood. Plates were changed regularly in between. At day 1, 3 and 5 of adulthood, 30 worms were equally distributed to new NGM plates and the grinder movement of the terminal bulb within the worm pharynx was counted for one minute under binocular observation. This assay was performed according to Raizen et al., 2005.

\subsubsection{Lifespan assay}

Worms were bleached and isolated eggs were transferred to RNAi plates seeded with oxy-4 RNAi, lmn-1 RNAi or L4440 (empty vector) expressing bacteria. Approximately 100 L4 larvae were separated with ongoing RNAi treatment and survival rate was determined every second day. Animals were considered as dead when they did not longer react after gently touching with a platinum wire. Dead and missing worms were considered as censored. Lifespan analysis were performed at $20^{\circ} \mathrm{C}$.

\subsubsection{Microinjection of C.elegans}

To fix worms for injection procedure, agarose pads were prepared. Therefore $2 \%$ of agarose were dissolved in water, boiled and $100 \mu \mathrm{l}$ of hot agerose were placed to a glass slide and quickly covered by a cover slip. The agarose was let solidify and prepared slides were stored till usage.

For the generation of transgenic worms, young hermaphrodites were picked and transformed to the agarose pads and the worms were covered with halocarbon oil 700 to prevent drying. Afterwards, an injection-mix, consisting of a visible marker and the desired DNA was injected into one or both of the gonad arms. The concentrations were ranging from $5 \mathrm{ng} / \mu \mathrm{l}$ - $125 \mathrm{ng} / \mu \mathrm{l}$ for Baf-1P-GFP oxy-4 and $10 \mathrm{ng} / \mu \mathrm{l}$ - $100 \mathrm{ng} / \mu \mathrm{l}$ for the marker. The injected worms were recovered and released from the agarose pad by adding a drop of M9 buffer and transferred separately to a fresh NGM plate using a platinum wire. The worms were grown at $20^{\circ} \mathrm{C}$ and the offspring were scored by fluorescence for successful injection.

\subsubsection{Microscopy}

\subsubsection{Fluorescence recovery after photobleaching (FRAP)}

In order to determine the turnover rate of GFP-Kuk (C567S) under control of the endogenous promoter, a circular area at the surface of GFP expressing embryos during 
cellularization was bleached using 100\% laser power, 15 iterations at a scan speed of 6 . To compare GFP-Kuk and a non-farnesylabale mutant GFP-kukC567S, embryos at a similar stage of cellularization were choosen. Fluorescence of two areas outside and one area inside of the bleached region were measured to determine the background signal. Subsequent analysis was done by using ImageJ. 


\section{References}

Araújo, Ana Rita; Reis, Micael; Rocha, Helder; Aguiar, Bruno; Morales-Hojas, Ramiro; Macedo-Ribeiro, Sandra et al. (2013): The Drosophila melanogaster methuselah gene. A novel gene with ancient functions. In: PloS one 8 (5), e63747.

Bahadorani, Sepehr; Hur, Jae H.; Lo Jr, Thomas; Vu, Kevin; Walker, David W. (2010): Perturbation of mitochondrial complex $\mathrm{V}$ alters the response to dietary restriction in Drosophila. In: Aging cell 9 (1), S. 100-103.

Balk, J.; Pierik, A. J.; Netz, D. AguilarJ; Mühlenhoff, U.; Lill, R. (2005): Nar1p, a conserved eukaryotic protein with similarity to Fe-only hydrogenases, functions in cytosolic ironsulphur protein biogenesis: Portland Press Limited.

Balk, Janneke; Pierik, Antonio J.; Netz, Daili J Aguilar; Mühlenhoff, Ulrich; Lill, Roland (2004): The hydrogenase-like Nar1p is essential for maturation of cytosolic and nuclear ironsulphur proteins. In: The EMBO journal 23 (10), S. 2105-2115.

Bank, Erin M.; Ben-Harush, Kfir; Feinstein, Naomi; Medalia, Ohad; Gruenbaum, Yosef (2012): Structural and physiological phenotypes of disease-linked lamin mutations in C. elegans. In: Journal of Structural Biology 177 (1), S. 106-112.

Bar, Daniel Z.; Gruenbaum, Yosef (2010): Reversal of age-dependent nuclear morphology by inhibition of prenylation does not affect lifespan in Caenorhabditis elegans. In: Nucleus 1 (6), S. 499-505.

Bar, Daniel Z.; Neufeld, Ester; Feinstein, Naomi; Gruenbaum, Yosef (2009): Gliotoxin reverses age-dependent nuclear morphology phenotypes, ameliorates motility, but fails to affect lifespan of adult Caenorhabditis elegans. In: Cell motility and the cytoskeleton 66 (10), S. 791-797.

Barton, Racine M.; Worman, Howard J. (1999): Prenylated prelamin A interacts with Narf, a novel nuclear protein. In: Journal of Biological Chemistry 274 (42), S. 30008-30018.

Beumer, Kelly J.; Trautman, Jonathan K.; Mukherjee, Kusumika; Carroll, Dana (2013): Donor DNA utilization during gene targeting with zinc-finger nucleases. In: G3: Genes, Genomes, Genetics 3 (4), S. 657-664. 
Bischof, Johannes; Björklund, Mikael; Furger, Edy; Schertel, Claus; Taipale, Jussi; Basler, Konrad (2013): A versatile platform for creating a comprehensive UAS-ORFeome library in Drosophila. In: Development 140 (11), S. 2434-2442.

Bischof, Johannes; Maeda, Robert K.; Hediger, Monika; Karch, François; Basler, Konrad (2007): An optimized transgenesis system for Drosophila using germ-line-specific IvarphiC31 integrases. In: Proceedings of the National Academy of Sciences 104 (9), S. 3312-3317.

Biteau, Benoit; Karpac, Jason; Supoyo, Stephen; DeGennaro, Matthew; Lehmann, Ruth; Jasper, Heinrich (2010): Lifespan extension by preserving proliferative homeostasis in Drosophila. In: PLoS genetics 6 (10), e1001159.

Bossie, Cynthia A.; Sanders, Marilyn M. (1993): A cDNA from Drosophila melanogaster encodes a lamin C-like intermediate filament protein. In: Journal of Cell Science 104 (4), S. $1263-1272$.

Brandt, Annely; Krohne, Georg; Großhans, Jörg (2008): The farnesylated nuclear proteins KUGELKERN and LAMIN B promote aging-like phenotypes in Drosophila flies. In: Aging cell 7 (4), S. 541-551.

Brandt, Annely; Papagiannouli, Fani; Wagner, Nicole; Wilsch-Bräuninger, Michaela; Braun, Martina; Furlong, Eileen E. et al. (2006): Developmental control of nuclear size and shape by Kugelkern and Kurzkern. In: Current biology 16 (6), S. 543-552.

Brandt, Annely; Vilcinskas, Andreas (2013): The fruit fly Drosophila melanogaster as a model for aging research. In: Yellow Biotechnology I: Springer, S. 63-77.

Brent, Roger; Ptashne, Mark (1985): A eukaryotic transcriptional activator bearing the DNA specificity of a prokaryotic repressor. In: Cell 43 (3), S. 729-736.

Broers, J. L.V.; Ramaekers, F. C.S.; Bonne, G.; Yaou, R. Ben; Hutchison, C. J. (2006): Nuclear lamins. Laminopathies and their role in premature aging. In: Physiological Reviews 86 (3), S. 967-1008.

Bruick, Richard K.; McKnight, Steven L. (2001): A conserved family of prolyl-4hydroxylases that modify HIF. In: Science 294 (5545), S. 1337-1340.

Capell, Brian C.; Erdos, Michael R.; Madigan, James P.; Fiordalisi, James J.; Varga, Renee; Conneely, Karen N. et al. (2005): Inhibiting farnesylation of progerin prevents the 
characteristic nuclear blebbing of Hutchinson-Gilford progeria syndrome. In: Proceedings of the National Academy of Sciences of the United States of America 102 (36), S. 1287912884.

Cavazza, Christine; Martin, Lydie; Mondy, Samuel; Gaillard, Jacques; Ratet, Pascal; Fontecilla-Camps, Juan C. (2008): The possible role of an [FeFe]-hydrogenase-like protein in the plant responses to changing atmospheric oxygen levels. In: Journal of inorganic biochemistry 102 (5), S. 1359-1365.

Chevaillier, Philippe (1993): Pest sequences in nuclear proteins. In: International journal of biochemistry 25 (4), S. 479-482.

Chung, Henry; Sztal, Tamar; Pasricha, Shivani; Sridhar, Mohan; Batterham, Philip; Daborn, Phillip J. (2009): Characterization of Drosophila melanogaster cytochrome P450 genes. In: Proceedings of the National Academy of Sciences 106 (14), S. 5731-5736.

Copeland, Jeffrey M.; Cho, Jaehyoung; Lo, Thomas; Hur, Jae H.; Bahadorani, Sepehr; Arabyan, Tagui et al. (2009): Extension of Drosophila life span by RNAi of the mitochondrial respiratory chain. In: Current Biology 19 (19), S. 1591-1598.

Corbin, Monique V.; Rockx, Davy A. P.; Oostra, Anneke B.; Joenje, Hans; Dorsman, Josephine C. (2015): The iron-sulfur cluster assembly network component NARFL is a key element in the cellular defense against oxidative stress. In: Free Radical Biology and Medicine 89, S. 863-872.

Coulthard, Alistair B.; Alm, Christina; Cealiac, Iulia; Sinclair, Don A.; Honda, Barry M.; Rossi, Fabrizio et al. (2010): Essential loci in centromeric heterochromatin of Drosophila melanogaster. I: the right arm of chromosome 2. In: Genetics 185 (2), S. 479-495.

Dechat, Thomas; Adam, Stephen A.; Taimen, Pekka; Shimi, Takeshi; Goldman, Robert D. (2010): Nuclear lamins. In: Cold Spring Harbor perspectives in biology 2 (11), a000547.

Dechat, Thomas; Pfleghaar, Katrin; Sengupta, Kaushik; Shimi, Takeshi; Shumaker, Dale K.; Solimando, Liliana; Goldman, Robert D. (2008): Nuclear lamins. Major factors in the structural organization and function of the nucleus and chromatin. In: Genes \& development 22 (7), S. 832-853.

Dechat, Thomas; Shimi, Takeshi; Adam, Stephen A.; Rusinol, Antonio E.; Andres, Douglas A.; Spielmann, H. Peter et al. (2007): Alterations in mitosis and cell cycle progression caused 
by a mutant lamin A known to accelerate human aging. In: Proceedings of the National Academy of Sciences 104 (12), S. 4955-4960.

Dialynas, George; Shrestha, Om K.; Ponce, Jessica M.; Zwerger, Monika; Thiemann, Dylan A.; Young, Grant H. et al. (2015): Myopathic lamin mutations cause reductive stress and activate the nrf2/keap-1 pathway. In: PLoS genetics 11 (5), e1005231.

Dobrzynska, Agnieszka; Askjaer, Peter; Gruenbaum, Yosef (2016): Chapter Twenty-TwoLamin-Binding Proteins in Caenorhabditis elegans. In: Methods in enzymology 569, S. 455483.

Dong, Jinsheng; Lai, Ruby; Nielsen, Klaus; Fekete, Christie A.; Qiu, Hongfang; Hinnebusch, Alan G. (2004): The essential ATP-binding cassette protein RLI1 functions in translation by promoting preinitiation complex assembly. In: Journal of Biological Chemistry 279 (40), S. 42157-42168.

Eckersley-Maslin, Melanie A.; Bergmann, Jan H.; Lazar, Zsolt; Spector, David L. (2013): Lamin A/C is expressed in pluripotent mouse embryonic stem cells. In: nucleus 4 (1), S. 5360 .

Erber, Andreas; Riemer, Dieter; Hofemeister, Helmut; Bovenschulte, Marc; Stick, Reimer; Panopoulou, Georgia et al. (1999): Characterization of the Hydra lamin and its gene. A molecular phylogeny of metazoan lamins. In: Journal of molecular evolution 49 (2), S. 260 271.

Eriksson, Maria; Brown, W. Ted; Gordon, Leslie B.; Glynn, Michael W.; Singer, Joel; Scott, Laura et al. (2003): Recurrent de novo point mutations in lamin A cause Hutchinson-Gilford progeria syndrome.

Fields, Stanley; Song, Ok-kyu (1989): A novel genetic system to detect protein-protein interactions. In: Nature 340 (6230), S. 245-246.

Fujii, Michihiko; Adachi, Noritaka; Shikatani, Kazuki; Ayusawa, Dai (2009): [FeFe]hydrogenase-like gene is involved in the regulation of sensitivity to oxygen in yeast and nematode. In: Genes to Cells 14 (4), S. 457-468.

Gargano, Julia Warner; Martin, Ian; Bhandari, Poonam; Grotewiel, Michael S. (2005): Rapid iterative negative geotaxis (RING). A new method for assessing age-related locomotor decline in Drosophila. In: Experimental gerontology 40 (5), S. 386-395. 
Gari, Kerstin; Ortiz, Ana Marl’ia León; Borel, Valérie; Flynn, Helen; Skehel, J. Mark; Boulton, Simon J. (2012): MMS19 links cytoplasmic iron-sulfur cluster assembly to DNA metabolism. In: Science 337 (6091), S. 243-245.

Gilchrist, Susan; Gilbert, Nick; Perry, Paul; Östlund, Cecilia; Worman, Howard J.; Bickmore, Wendy A. (2004): Altered protein dynamics of disease-associated lamin A mutants. In: BMC cell Biology 5 (1), S. 46.

Gonzalez-Suarez, Ignacio; Redwood, Abena B.; Gonzalo, Susana (2009): Loss of A-type lamins and genomic instability. In: Cell Cycle 8 (23), S. 3860-3865. In: PNAS 109 (41), S. 16666-16671.

Gordon, L. B., Kleinman, M. E., Miller, D. T., Neuberg, D. S., et al. (2013). Clinical trial of a farnesyltransferase inhibitor in children with Hutchinson-Gilford progeria syndrome.

Gosh, S. and Zhou, Z. (2014) Genetics of aging, progeria and lamin disorders. Curr Opin Genet Dev. 26, S. 41-46.

Gratz, Scott J.; Ukken, Fiona P.; Rubinstein, C. Dustin; Thiede, Gene; Donohue, Laura K.; Cummings, Alexander M.; O’Connor-Giles, Kate M. (2014): Highly specific and efficient CRISPR/Cas9-catalyzed homology-directed repair in Drosophila. In: Genetics 196 (4), S. 961-971.

Gruenbaum, Yosef; Foisner, Roland (2015): Lamins. Nuclear intermediate filament proteins with fundamental functions in nuclear mechanics and genome regulation. In: Annu. Rev. Biochem. 84, S. 131-164.

Gruenbaum, Yosef; Goldman, Robert D.; Meyuhas, Ronit; Mills, Erez; Margalit, Ayelet; Fridkin, Alexandra et al. (2003): The nuclear lamina and its functions in the nucleus. In: International review of cytology 226, S. 1-62.

Gruenbaum, Yosef; Landesman, Yosef; Drees, Barry; Bare, John W.; Saumweber, Harald; Paddy, Michael R. et al. (1988): Drosophila nuclear lamin precursor Dm0 is translated from either of two developmentally regulated mRNA species apparently encoded by a single gene. In: The Journal of cell biology 106 (3), S. 585-596.

Hackstein, J. H.P. (2005): Eukaryotic Fe-hydrogenases-old eukaryotic heritage or adaptive acquisitions?: Portland Press Limited. 
Haithcock, Erin; Dayani, Yaron; Neufeld, Ester; Zahand, Adam J.; Feinstein, Naomi; Mattout, Anna et al. (2005): Age-related changes of nuclear architecture in Caenorhabditis elegans. In: Proceedings of the National Academy of Sciences of the United States of America 102 (46), S. 16690-16695.

He, Ying; Jasper, Heinrich (2014): Studying aging in Drosophila. In: Methods 68 (1), S. 129-133.

Huang, Jianhe; Song, Daisheng; Flores, Adrian; Zhao, Quan; Mooney, Sharon M.; Shaw, Leslie M.; Lee, Frank S. (2007): IOP1, a novel hydrogenase-like protein that modulates hypoxia-inducible factor-1\alpha activity. In: Biochemical Journal 401 (1), S. 341-352.

Ivan, Mircea; Kondo, Keiichi; Yang, Haifeng; Kim, William; Valiando, Jennifer; Ohh, Michael et al. (2001): HIFlalpha targeted for VHL-mediated destruction by proline hydroxylation: implications for O2 sensing. In: Science 292 (5516), S. 464-468.

Jaakkola, Panu; Mole, David R.; Tian, Ya-Min; Wilson, Michael I.; Gielbert, Janine; Gaskell, Simon J. et al. (2001): Targeting of HIF-lalpha to the von Hippel-Lindau ubiquitylation complex by O2-regulated prolyl hydroxylation. In: Science 292 (5516), S. 468-472.

Jain, Ashish; Lamark, Trond; Sjøttem, Eva; Larsen, Kenneth Bowitz; Awuh, Jane Atesoh; Øvervatn, Aud et al. (2010): p62/SQSTM1 is a target gene for transcription factor NRF2 and creates a positive feedback loop by inducing antioxidant response element-driven gene transcription. In: Journal of Biological Chemistry 285 (29), S. 22576-22591.

Jiang, Huaqi; Patel, Parthive H.; Kohlmaier, Alexander; Grenley, Marc O.; McEwen, Donald G.; Edgar, Bruce A. (2009): Cytokine/Jak/Stat signaling mediates regeneration and homeostasis in the Drosophila midgut. In: Cell 137 (7), S. 1343-1355.

Jinek, Martin; Chylinski, Krzysztof; Fonfara, Ines; Hauer, Michael; Doudna, Jennifer A.; Charpentier, Emmanuelle (2012): A programmable dual-RNA-guided DNA endonuclease in adaptive bacterial immunity. In: Science 337 (6096), S. 816-821.

Johnson, Deborah C.; Dean, Dennis R.; Smith, Archer D.; Johnson, Michael K. (2005): Structure, function, and formation of biological iron-sulfur clusters. In: Annu. Rev. Biochem. 74, S. 247-281. 
Kispal, Gyula; Sipos, Katalin; Lange, Heike; Fekete, Zsuzsanna; Bedekovics, Tibor; Janáky, Tamás et al. (2005): Biogenesis of cytosolic ribosomes requires the essential iron-sulphur protein Rli1p and mitochondria. In: The EMBO journal 24 (3), S. 589-598.

Kitten, G. T.; Nigg, E. A. (1991): The CaaX motif is required for isoprenylation, carboxyl methylation, and nuclear membrane association of lamin B2. In: The Journal of cell biology 113 (1), S. 13-23.

Kolb, Thorsten; Maaß, Kendra; Hergt, Michaela; Aebi, Ueli; Herrmann, Harald (2011): Lamin A and lamin C form homodimers and coexist in higher complex forms both in the nucleoplasmic fraction and in the lamina of cultured human cells. In: nucleus 2 (5), S. 425433.

Komatsu, Masaaki; Kurokawa, Hirofumi; Waguri, Satoshi; Taguchi, Keiko; Kobayashi, Akira; Ichimura, Yoshinobu et al. (2010): The selective autophagy substrate p62 activates the stress responsive transcription factor Nrf2 through inactivation of Keap1. In: Nature cell biology 12 (3), S. 213-223.

Kondo, Shu; Ueda, Ryu (2013): Highly improved gene targeting by germline-specific Cas9 expression in Drosophila. In: Genetics 195 (3), S. 715-721.

Krohne, G. (1998): Lamin assembly in vivo. In: Sub-cellular biochemistry 31, S. 563-586. Lieber, Michael R. (2010): The mechanism of double-strand DNA break repair by the nonhomologous DNA end-joining pathway. In: Annu. Rev. Biochem. 79, S. 181-211.

Lill, Roland (2009): Function and biogenesis of iron-sulphur proteins. In: Nature 460 (7257), S. 831-838.

Lin, Yi-Jyun; Seroude, Laurent; Benzer, Seymour (1998): Extended life-span and stress resistance in the Drosophila mutant methuselah. In: Science 282 (5390), S. 943-946.

Liu, Yiyong; Rusinol, Antonio; Sinensky, Michael; Wang, Youjie; Zou, Yue (2006): DNA damage responses in progeroid syndromes arise from defective maturation of prelamin A. In: J Cell Sci 119 (22), S. 4644-4649.

Loboda, Agnieszka; Damulewicz, Milena; Pyza, Elzbieta; Jozkowicz, Alicja; Dulak, Jozef (2016): Role of Nrf2/HO-1 system in development, oxidative stress response and diseases. An evolutionarily conserved mechanism. In: Cellular and Molecular Life Sciences 73 (17), S. 3221-3247. 
McClintock, Dayle; Ratner, Desiree; Lokuge, Meepa; Owens, David M.; Gordon, Leslie B.; Collins, Francis S.; Djabali, Karima (2007): The mutant form of lamin A that causes Hutchinson-Gilford progeria is a biomarker of cellular aging in human skin. In: PLoS One 2 (12), e1269.

Mendoza, Alexandre de; Jones, Jeffery W.; Friedrich, Markus (2016): Methuselah/Methuselah-like G protein-coupled receptors constitute an ancient metazoan gene family. In: Scientific reports 6.

Meneely, Philip (2014): Genetic Analysis: Genes, Genomes, and Networks in Eukaryotes: Oxford University Press.

Misra, Jyoti R.; Horner, Michael A.; Lam, Geanette; Thummel, Carl S. (2011): Transcriptional regulation of xenobiotic detoxification in Drosophila. In: Genes \& development 25 (17), S. 1796-1806.

Moir, Robert D.; Yoon, Miri; Khuon, Satya; Goldman, Robert D. (2000): Nuclear Lamins a and B1. In: The Journal of cell biology 151 (6), S. 1155-1168.

Mondy, Samuel; Lenglet, Aurore; Cosson, Viviane; Pelletier, Sandra; Pateyron, Stéphanie; Gilard, Françoise et al. (2014): GOLLUM [FeFe]-hydrogenase-like proteins are essential for plant development in normoxic conditions and modulate energy metabolism. In: Plant, cell \& environment 37 (1), S. 54-69.

Motohashi, Hozumi; Yamamoto, Masayuki (2004): Nrf2-Keap1 defines a physiologically important stress response mechanism. In: Trends in molecular medicine 10 (11), S. 549557.

Moulson, Casey L.; Fong, Loren G.; Gardner, Jennifer M.; Farber, Emily A.; Go, Gloriosa; Passariello, Annalisa et al. (2007): Increased progerin expression associated with unusual LMNA mutations causes severe progeroid syndromes. In: Human mutation 28 (9), S. 882889.

Muñoz-Alarcón, Andrés; Pavlovic, Maja; Wismar, Jasmine; Schmitt, Bertram; Eriksson, Maria; Kylsten, Per; Dushay, Mitchell S. (2007): Characterization of lamin mutation phenotypes in Drosophila and comparison to human laminopathies. In: PLoS One 2 (6), e532.

Nakamura, Miyuki; Buzas, Diana Mihaela; Kato, Akira; Fujita, Masahiro; Kurata, Nori; Kinoshita, Tetsu (2013): The role of Arabidopsis thaliana NAR1, a cytosolic iron-sulfur 
cluster assembly component, in gametophytic gene expression and oxidative stress responses in vegetative tissue. In: New Phytologist 199 (4), S. 925-935.

Nicholson, Louise; Singh, Gunisha K.; Osterwalder, Thomas; Roman, Gregg W.; Davis, Ronald L.; Keshishian, Haig (2008): Spatial and temporal control of gene expression in Drosophila using the inducible GeneSwitch GAL4 system. I. Screen for larval nervous system drivers. In: Genetics 178 (1), S. 215-234.

Nicolet, Yvain; Cavazza, Christine; Fontecilla-Camps, J. C. (2002): Fe-only hydrogenases: structure, function and evolution. In: Journal of inorganic biochemistry 91 (1), S. 1-8.

Pendás, Alberto M.; Zhou, Zhongjun; Cadiñanos, Juan; Freije, José M. P.; Wang, Jianming; Hultenby, Kjell et al. (2002): Defective prelamin A processing and muscular and adipocyte alterations in Zmpste24 metalloproteinase-deficient mice. In: Nature genetics 31 (1), S. 9499.

Pesavento, Patricia A.; Stewart, Russell J.; Goldstein, L. S. (1994): Characterization of the KLP68D kinesin-like protein in Drosophila. Possible roles in axonal transport. In: The Journal of cell biology 127 (4), S. 1041-1048.

Pollex, R. L.; Hegele, R. A. (2004): Hutchinson-Gilford progeria syndrome. In: Clinical genetics 66 (5), S. 375-381.

Progeria Research Foundation (PRF) webpage http://www.progeriaresearch.org/

Rahman, Mohammed Mahidur; Sykiotis, Gerasimos P.; Nishimura, Mayuko; Bodmer, Rolf; Bohmann, Dirk (2013): Declining signal dependence of Nrf2-MafS-regulated gene expression correlates with aging phenotypes. In: Aging cell 12 (4), S. 554-562.

Raizen, David; Song, Bo-mi; Trojanowski, Nick; You, Young-Jai (2005): Methods for measuring pharyngeal behaviors.

Ray, Krishanu; Perez, Sharon E.; Yang, Zhaohuai; Xu, Jenny; Ritchings, Bruce W.; Steller, Hermann; Goldstein, Lawrence S. B. (1999): Kinesin-II is required for axonal transport of choline acetyltransferase in Drosophila. In: The Journal of cell biology 147 (3), S. 507-518. Rechsteiner, Martin; Rogers, Scott W. (1996): PEST sequences and regulation by proteolysis. In: Trends in biochemical sciences 21 (7), S. 267-271. 
Riemer, Dieter; Stuurman, Nico; Berrios, Miguel; Hunter, Cecil; Fisher, Paul A.; Weber, Klaus (1995): Expression of Drosophila lamin C is developmentally regulated. Analogies with vertebrate A-type lamins. In: Journal of Cell Science 108 (10), S. 3189-3198.

Rogers, Scott; Wells, Rodney; Rechsteiner, Martin (1986): Amino acid sequences common to rapidly degrade proteins. The PEST hypothesis. In: Science 234, S. 364-369.

Rudolf, Jana; Makrantoni, Vasso; Ingledew, W. John; Stark, Michael, JR; White, Malcolm F. (2006): The DNA repair helicases XPD and FancJ have essential iron-sulfur domains. In: Molecular cell 23 (6), S. 801-808.

Rusiñol, Antonio E.; Sinensky, Michael S. (2006): Farnesylated lamins, progeroid syndromes and farnesyl transferase inhibitors. In: Journal of Cell Science 119 (16), S. 32653272.

Salceda, Susana; Caro, Jaime (1997): Hypoxia-inducible Factor 1\alpha (HIF-1\alpha) Protein Is Rapidly Degraded by the Ubiquitin-Proteasome System under Normoxic Conditions ITS STABILIZATION BY HYPOXIA DEPENDS ON REDOX-INDUCED CHANGES. In: Journal of Biological Chemistry 272 (36), S. 22642-22647.

Sambrook, Joseph; Russell, David W. (2001): Molecular cloning. A laboratory manual. 2001: Cold Spring Harbor Laboratory Press, Cold Spring Harbor, New York.

Sandre-Giovannoli, Annachiara de; Bernard, Rafaelle; Cau, Pierre; Navarro, Claire; Amiel, Jeanne; Boccaccio, Irene et al. (2003): Lamin a truncation in Hutchinson-Gilford progeria. In: Science 300 (5628), S. 2055.

Scaffidi, Paola; Misteli, Tom (2005): Reversal of the cellular phenotype in the premature aging disease Hutchinson-Gilford progeria syndrome. In: Nature medicine 11 (4), S. 440445.

Schulze, Sandra R.; Curio-Penny, Beatrice; Speese, Sean; Dialynas, George; Cryderman, Diane E.; McDonough, Caitrin W. et al. (2009): A comparative study of Drosophila and human A-type lamins. In: PLoS One 4 (10), e7564.

Scott, Jeffrey G.; Wen, Zhimou (2001): Cytochromes P450 of insects. The tip of the iceberg. In: Pest management science 57 (10), S. 958-967.

Semenza, Gregg L. (1999): Regulation of mammalian O2 homeostasis by hypoxia-inducible factor 1. In: Annual review of cell and developmental biology 15 (1), S. 551-578. 
Short, Jay M.; Fernandez, Joseph M.; Sorge, Joseph A.; Huse, William D. (1988): łambda ZAP. A bacteriophage łambda expression vector with in vivo excision properties. In: Nucleic acids research 16 (15), S. 7583-7600.

Sinensky, Michael; Fantle, K.; Trujillo, M.; McLain, T.; Kupfer, A.; Dalton, M. (1994): The processing pathway of prelamin A. In: Journal of Cell Science 107 (1), S. 61-67.

Song, Daisheng; Lee, Frank S. (2008): A role for IOP1 in mammalian cytosolic iron-sulfur protein biogenesis. In: Journal of Biological Chemistry 283 (14), S. 9231-9238.

Song, Daisheng; Lee, Frank S. (2011): Mouse knock-out of IOP1 protein reveals its essential role in mammalian cytosolic iron-sulfur protein biogenesis. In: Journal of Biological Chemistry 286 (18), S. 15797-15805.

Song, Daisheng; Tu, Zheng; Lee, Frank S. (2009): Human ISCA1 interacts with IOP1/NARFL and functions in both cytosolic and mitochondrial iron-sulfur protein biogenesis. In: Journal of Biological Chemistry 284 (51), S. 35297-35307.

Sono, Masanori; Roach, Mark P.; Coulter, Eric D.; Dawson, John H. (1996): Hemecontaining oxygenases. In: Chemical Reviews 96 (7), S. 2841-2888.

Stehling, Oliver; Vashisht, Ajay A.; Mascarenhas, Judita; Jonsson, Zophonias O.; Sharma, Tanu; Netz, Daili J. A. et al. (2012): MMS19 assembles iron-sulfur proteins required for DNA metabolism and genomic integrity. In: Science 337 (6091), S. 195-199.

Steinman, Howard M. (1980): The amino acid sequence of copper-zinc superoxide dismutase from bakers’ yeast. In: Journal of Biological Chemistry 255 (14), S. 6758-6765.

Sturtz, Lori A.; Diekert, Kerstin; Jensen, Laran T.; Lill, Roland; Culotta, Valeria Cizewski (2001): A fraction of yeast cu, zn-superoxide dismutase and its metallochaperone, ccs, localize to the intermembrane space of mitochondria a physiological role for sod1 in guarding against mitochondrial oxidative damage. In: Journal of Biological Chemistry 276 (41), S. 38084-38089.

Stuurman, Nico; Heins, Susanne; Aebi, Ueli (1998): Nuclear lamins. Their structure, assembly, and interactions. In: Journal of Structural Biology 122 (1-2), S. 42-66.

Sykiotis, Gerasimos P.; Bohmann, Dirk (2008): Keap1/Nrf2 signaling regulates oxidative stress tolerance and lifespan in Drosophila. In: Developmental cell 14 (1), S. 76-85. 
Tijet, Nathalie; Helvig, Christian; Feyereisen, René (2001): The cytochrome P450 gene superfamily in Drosophila melanogaster. Annotation, intron-exon organization and phylogeny. In: Gene 262 (1), S. 189-198.

Ullrich N. J., Kieran, M. W., Miller, D. T., Gordon, L. B., et al. (2013). Neurologic features of Hutchinson-Gilford progeria syndrome after lonafarnib treatment. Neurology 81 (5), S. 427-430.

Vidak, Sandra; Foisner, Roland (2016): Molecular insights into the premature aging disease progeria. In: Histochemistry and cell biology 145 (4), S. 401-417.

Weitkunat, Manuela; Schnorrer, Frank (2014): A guide to study Drosophila muscle biology. In: Methods 68 (1), S. 2-14.

Wiesel, Naama; Mattout, Anna; Melcer, Shai; Melamed-Book, Naomi; Herrmann, Harald; Medalia, Ohad et al. (2008): Laminopathic mutations interfere with the assembly, localization, and dynamics of nuclear lamins. In: Proceedings of the National Academy of Sciences 105 (1), S. 180-185.

Yamada, Ryuichi; Deshpande, Sonali A.; Keebaugh, Erin S.; Ehrlich, Margaux R.; Soto Obando, Alina; Ja, William W. (2017): Mifepristone reduces food palatability and affects Drosophila feeding and lifespan. In: The Journals of Gerontology: Series A 72 (2), S. 173180.

Yang, Jae-Seong; Nam, Hyun-Jun; Seo, Mihwa; Han, Seong Kyu; Choi, Yonghwan; Nam, Hong Gil et al. (2011): OASIS. Online application for the survival analysis of lifespan assays performed in aging research. In: PLoS One 6 (8), e23525.

Yarunin, Alexander; Panse, Vikram Govind; Petfalski, Elisabeth; Dez, Christophe; Tollervey, David; Hurt, Eduard C. (2005): Functional link between ribosome formation and biogenesis of iron-sulfur proteins. In: The EMBO journal 24 (3), S. 580-588.

Young, S. G., Yang, S. H., Davies, B. S., Jung, H. J., Fong, L. G. (2013) Targeting protein prenylation in progeria. Sci Transl Med. 5 (171): 171ps3

Young, Stephen G.; Fong, Loren G.; Michaelis, Susan; others (2005): Prelamin A, Zmpste24, misshapen cell nuclei, and progeria-new evidence suggesting that protein farnesylation could be important for disease pathogenesis. In: J Lipid Res 46 (12), S. 25312558. 
Young, Stephen G.; Meta, Margarita; Yang, Shao H.; Fong, Loren G. (2006): Prelamin A farnesylation and progeroid syndromes. In: Journal of Biological Chemistry 281 (52), S. 39741-39745.

Zaremba-Czogalla, Magdalena; Piekarowicz, Katarzyna; Wachowicz, Katarzyna; Kozioł, Katarzyna; Dubińska-Magiera, Magda; Rzepecki, Ryszard (2012): The different function of single phosphorylation sites of Drosophila melanogaster lamin Dm and lamin C. In: PLoS One 7 (2), e32649.

Zhao, Wei; Fang, Bing Xiong; Niu, Yu Jie; Liu, Yi Na; Liu, Bin; Peng, Qi et al. (2014): Nar1 deficiency results in shortened lifespan and sensitivity to paraquat that is rescued by increased expression of mitochondrial superoxide dismutase. In: Mechanisms of aging and development 138, S. 53-58.

Zuela, Noam; Zwerger, Monika; Levin, Tal; Medalia, Ohad; Gruenbaum, Yosef (2016): Impaired mechanical response of an EDMD mutation leads to motility phenotypes that are repaired by loss of prenylation. In: J Cell Sci 129 (9), S. 1781-1791. 


\title{
8 Supplement Data
}

\begin{abstract}
ATCACAAAGGTATATTGAAACAAAAAAAATTTAAAGATACATATTTATATGACATTTTTATATGTTTCTCGCG CATTATTTGTTTGTATCCAATTTATTATTTATTAGATAACGTTTATTAGTCTTAAATTAGTAGTCAATATTTC TACTCAACAAAACCCACAAAAGTCTATAATCACAAAAAATCAGTCAAATGCCATTTAAATAATTGGTTAGAGT ATGTAGCAATCCGGATAAAGTAAAATTGAACTTTCGCATTTATCTGGCATCGAGCTGTCTGGGAAAATTCCAT CTCTTGGCATTCTAGACGAATTTCTTCACTTCAGCCCatgatgaaataacataaggtggtccogtcggcaaga gacatccacttaacgtatgcttgcaataagtgcgagtgaaaggaatagtattctgagtgtcgtattgagtctg agtgagacagcgatatgattgttgattaacccttagcatgtccgtggggtttgaattaactcataagcttggc tgcaggtcgacctcgagggtaccaatgaacaggacctaacgcacagtcacgttattgtttacataaatgattt ttttactattcaaacttactctgtttgtgtactcccactggtatagccttctttatctttctggttcagg ctctatcaccggtttcaaaaaaaattcgtccgcacacaacctttcctctcaacaagcaaacgtgcactgaa tttaagtgtatacttcggtaagcttcggctatcgacgggaccaccttatgttatt tcatcatgGGCGGGACAA CGCCTAGTCCAAACGAACAGCGCCTGTCTGAACAACTTCCTCTTCCCCGTGCTGAGCGACATCCAGCGAAAGT ACTCCGGCGGAGGATCTGCAGGAAGCGGAGGATCAGGCGGCCGTAACATAGGCGTCGGCAGCGACATCGGCGA CCTGAAGTCTGTGTTCGAGCTGGCCGAACGTTCCAGTCCGGGAGTGAGCGACCTATTTATGAAAGAGCTGATC CACATGCTCGTTCCTGGTTACTCGGAGACGCGAATCAATACGATCATGGACCGCGCCATACGCAACCGCAAGT AGCTGGCGAAGCCGTAGCAAATTCCGATTCCAAGCAGCCTATTTTAATATATACACAACTTTATTAACGACAC CGAGACAACACAAACTGAAACGCTGGAGCAGACATATTCCGACATTTAGTGGCCAACAGAGGACCGGGAGCGA TGTACATGTATTTCAATTGAACAGTTAT
\end{abstract}

Supplement Figure 1: The deletion of $\Delta 15$ a kuk deficient fly line

The genomic breakpoints of the deletions were analyzed by PCR, including the neighboring genes of kuk. Final characterization was done by a long range PCR (MK26/MK29) followed by a subsequent sequencing analysis. The sequencing results revealed a depletion of $\sim 5.5 \mathrm{~kb}$ within the genomic locus of kuk (upper case) and GckIII (bold upper case). Furthermore, an insertion of 465 bp (lower case) due to initially performed transposon remobilization was found.

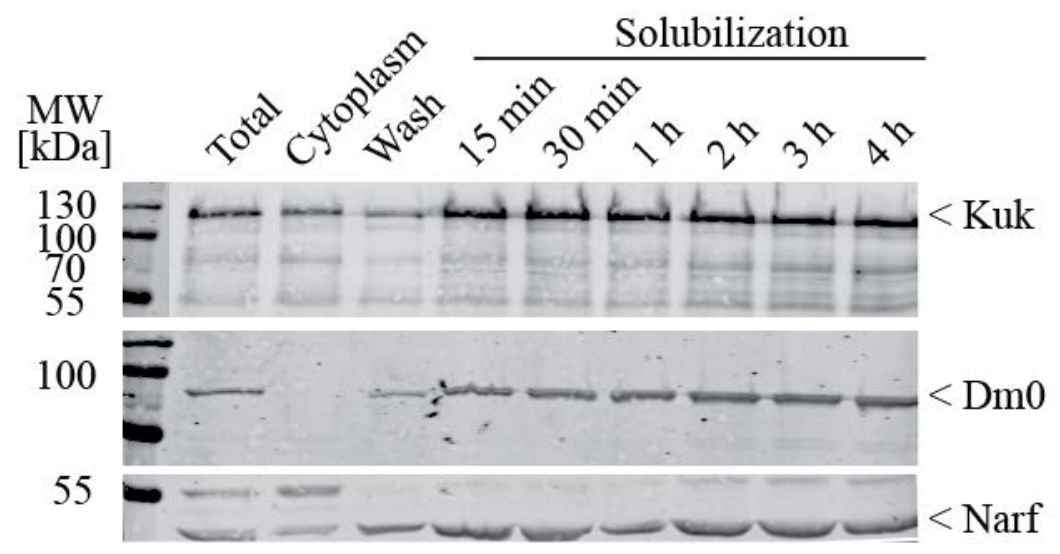

Supplement Figure 2: Kugelkern is stable for at least four hours after solubilization

Western blot analysis of nuclei extract incubated for maximal 4 hours with $150 \mathrm{mM} \mathrm{NaCl}$ and $1 \%$ TritonX100 for solubilization. A nuclei fractionation with $150 \mathrm{mg}$ wild type embryos was performed and a protein amount of 30 embryos was loaded for the total and the cytoplasm sample. The wash fraction was loaded after protein precipitation with trichloroacetic acid. For each time point after solubilization a sample with a theoretical protein amount of 2.250 embryos was taken and loaded after protein precipitation. 

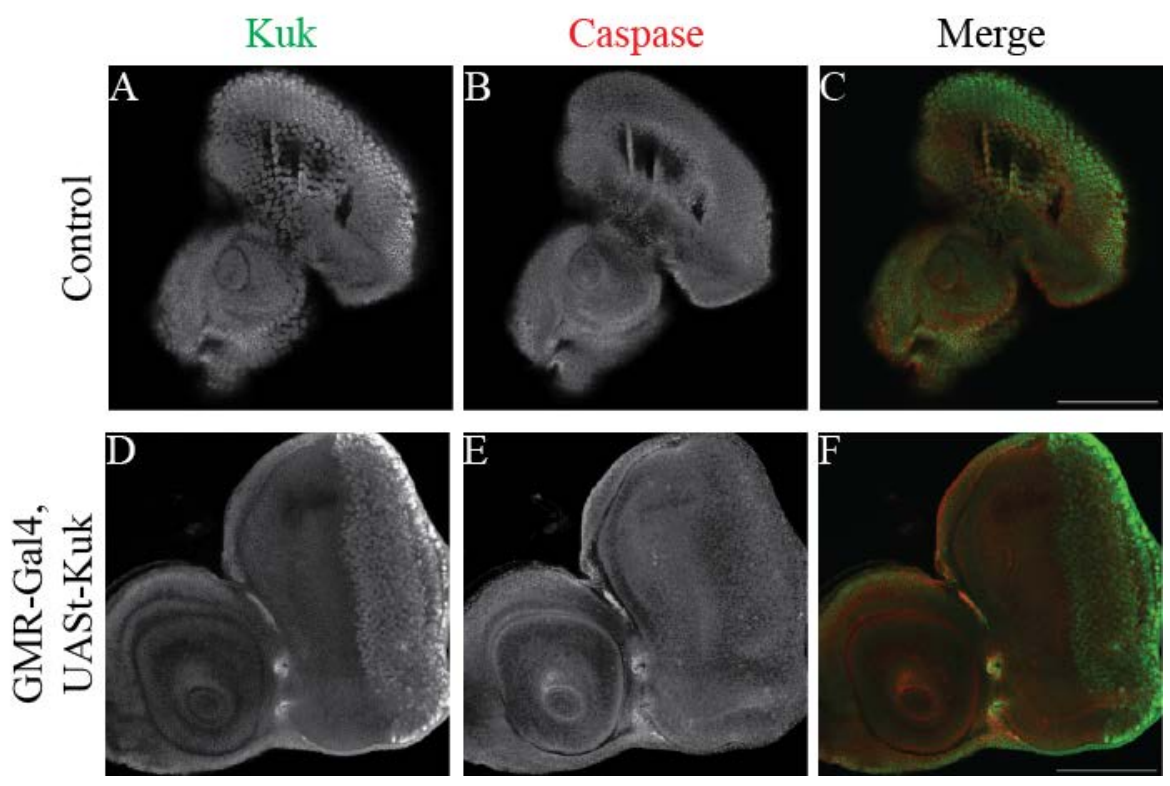

Supplement Figure 3: Kugelkern overexpression did not induce apoptosis in larval eye-discs Immunofluorescence stainings for Caspase-3 and Kugelkern in eye-antennal imaginal discs from L3 larvae. Discs from flies expressing GFP-cadherin under the ubiquitin promoter were used as control and stained in the same tube. Eye specific overexpression of Kugelkern elevated Kuk protein level (D) but did not increase the signal for activated Caspase (E) compared to control discs (A and B). Scale bar: $100 \mu \mathrm{m}$

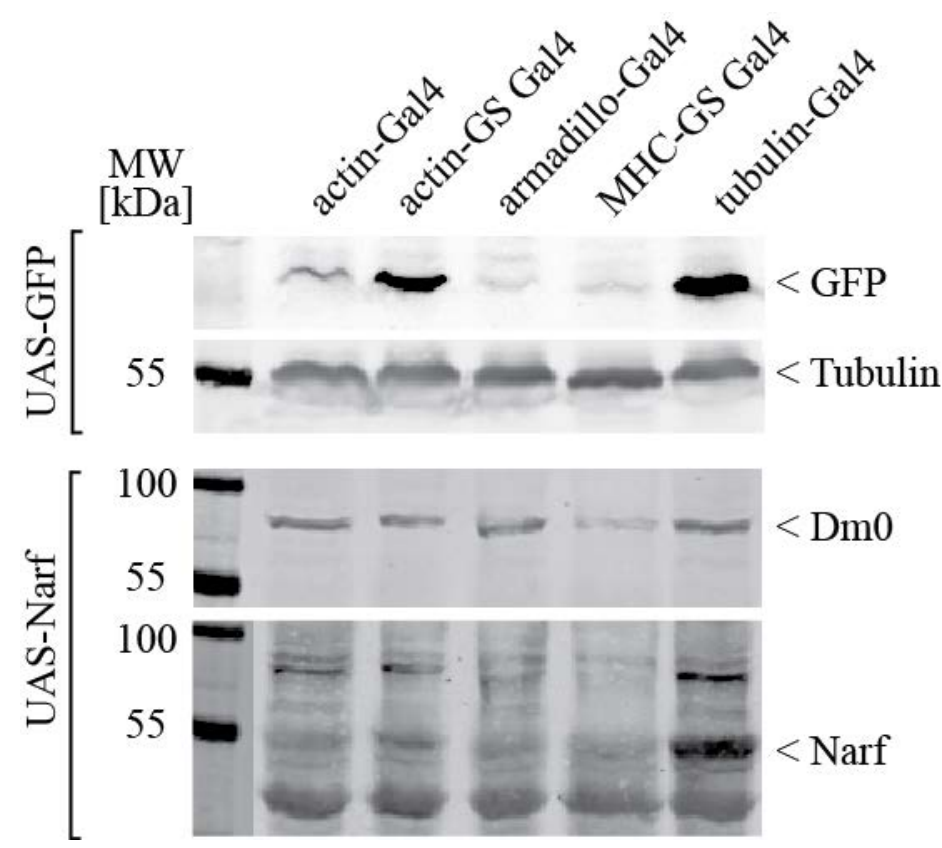

Supplement Figure 4: Strongest Narf expression was achieved using the tubulin-Gal4 driver Western blot analyses of total fly extract from GFP and Narf expressing animals controlled by different driver lines. Samples were taken after flies reached day 10 of adulthood. RU-dependent gene expression was induced with $20 \mu \mathrm{M}$ of RU for 7 days using 1-3 days old males. The lysate of one fly was loaded and stained for GFP 
and Narf. Dm0 and $\alpha$-tubulin were used as loading controls. The strongest Narf gene expression was achieved using tubulin-Gal4.

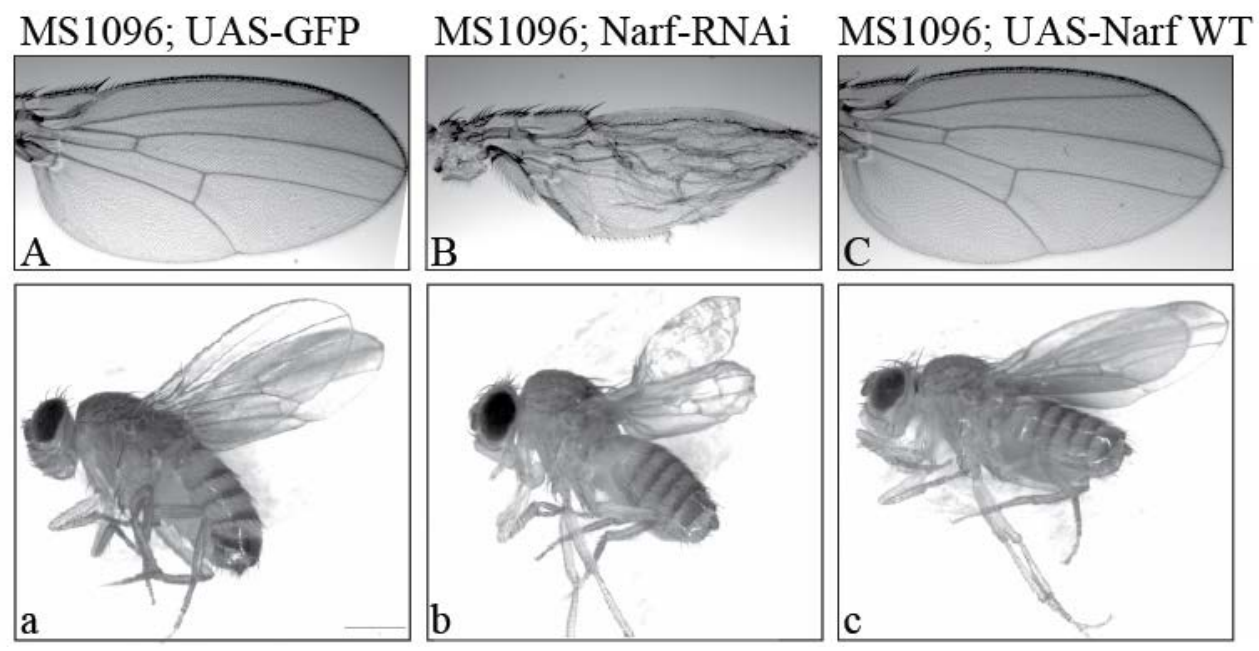

Supplement Figure 5: Wing specific down-regulation of Narf induced morphological defects Representative wing pictures of flies expressing UAS-GFP (A), Narf-RNAi (B) or UAS-Narf (C) wing specifically controlled by MS1096-Gal4. Expression of a long hairpin structure specific for Narf led to aberrant wing phenotypes (B) compared to GFP expressing control flies (A). An overexpression of Narf had no effects on wing development (C).

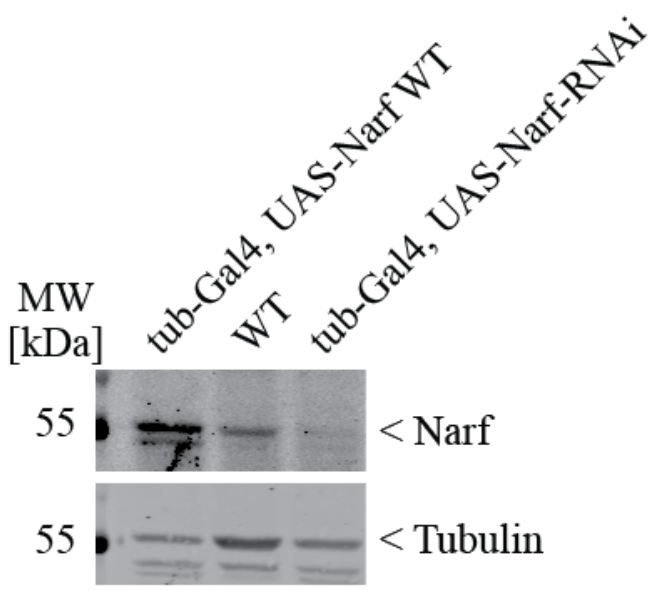

Supplement Figure 6: Narf-RNAi efficiently down regulated larval expressed Narf

Western blot analysis of larvae extract stained for Narf and $\alpha$-tubulin which was used as loading control. Ubiquitous expression of a long hairpin structure specific for Narf efficiently down regulated Narf protein level in L3 larvae compared to the wild type control. However, the larvae extract of the RNAi sample was a mix of RNAi expressing and non-expressing larvae extract as the used tubulin-Gal4 driver line was kept over a non GFP-tagged balancer chromosome. The lysate of 0.75 L3 larva was loaded. 


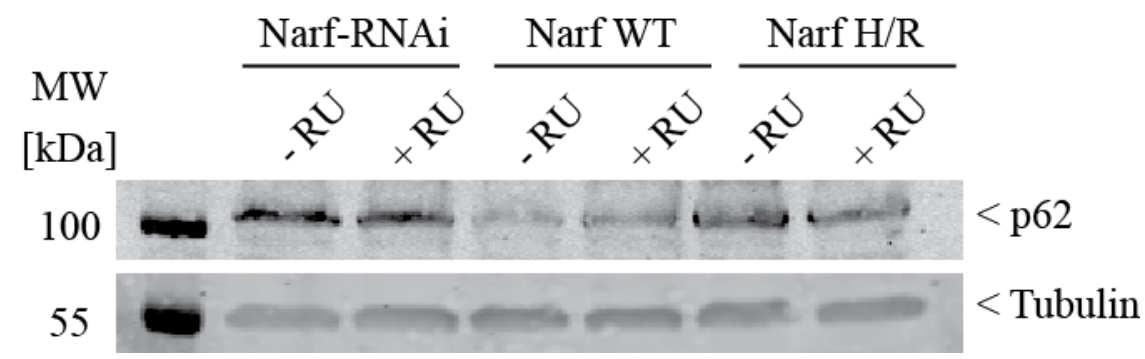

Supplement Figure 7: p62/Ref(2)P protein level is not affected upon altered Narf expression Western blot analyses of thorax samples from Narf-RNAi or UAS-Narf (WT or H/R) expressing flies controlled by the MHC-GS Gal driver line. The lysate of 1.5 thoraxes was loaded and stained for p62/Ref(2)P and $\alpha$-tubulin, which was used as loading control. RU-dependent gene expression was induced with $200 \mu \mathrm{M}$ of RU for 17 days using 1-3 days old males. In none of the tested situation an increase in protein level for p62 was detected compared to non-induced control flies.

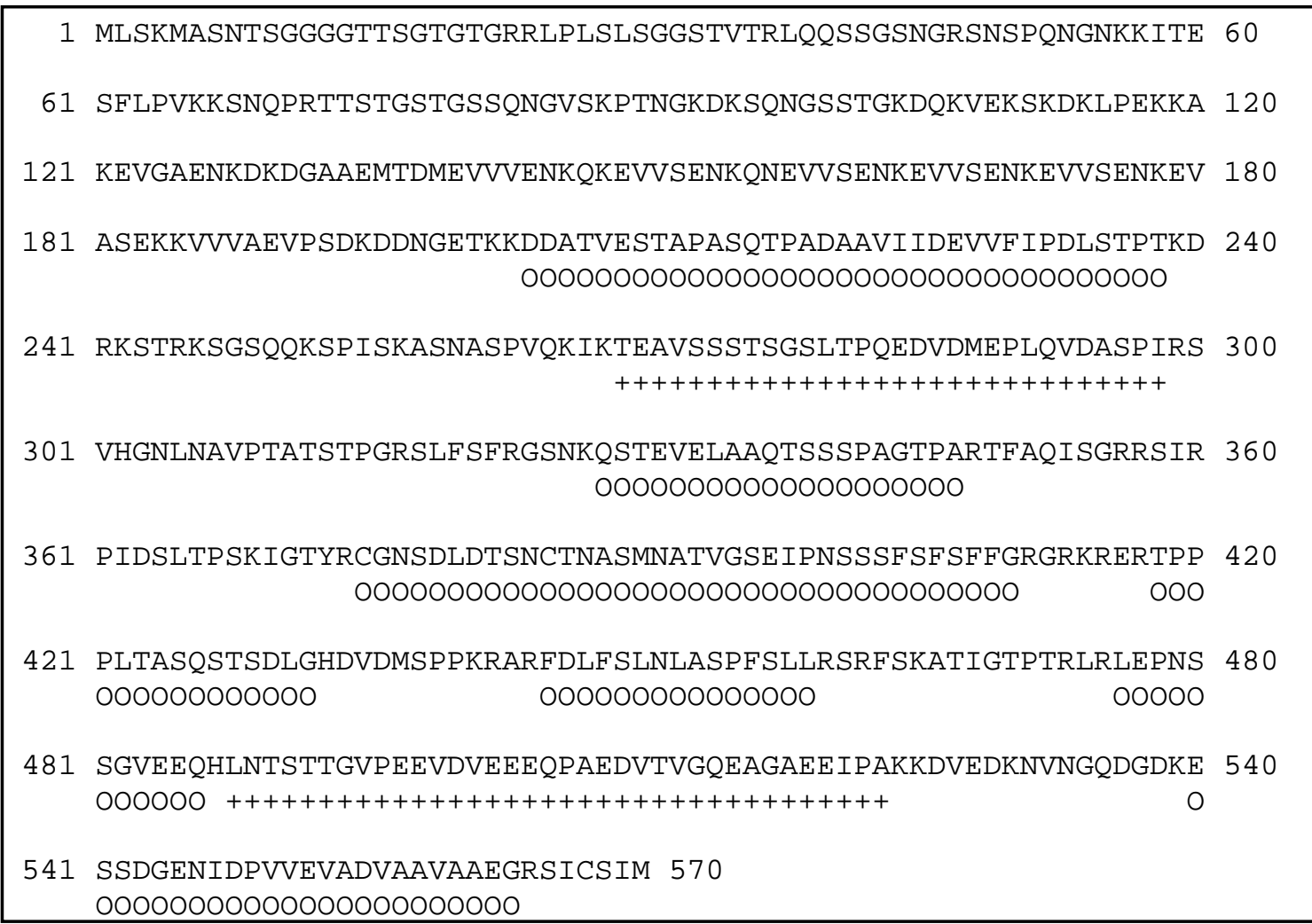

Supplement Figure 8: The amino acid sequence of Kuk contains two potential PEST-motifs Amino acid analysis of Kugelkern using the software epestfind led to the identification of nine PEST-motifs. Motifs indicated with a score value $>+5$ are potential PEST-sequences and indicated by a $(+)$. PEST-sequences with a score $<+5$ are below the threshold are termed as poor motifs ( 0 ). Kugelkern has two potential PESTmotifs with 30 amino acids between position 268 and 299 (PEST score: 7.24) and 36 amino acids between position 487 and 524 (PEST score: 9.56). 


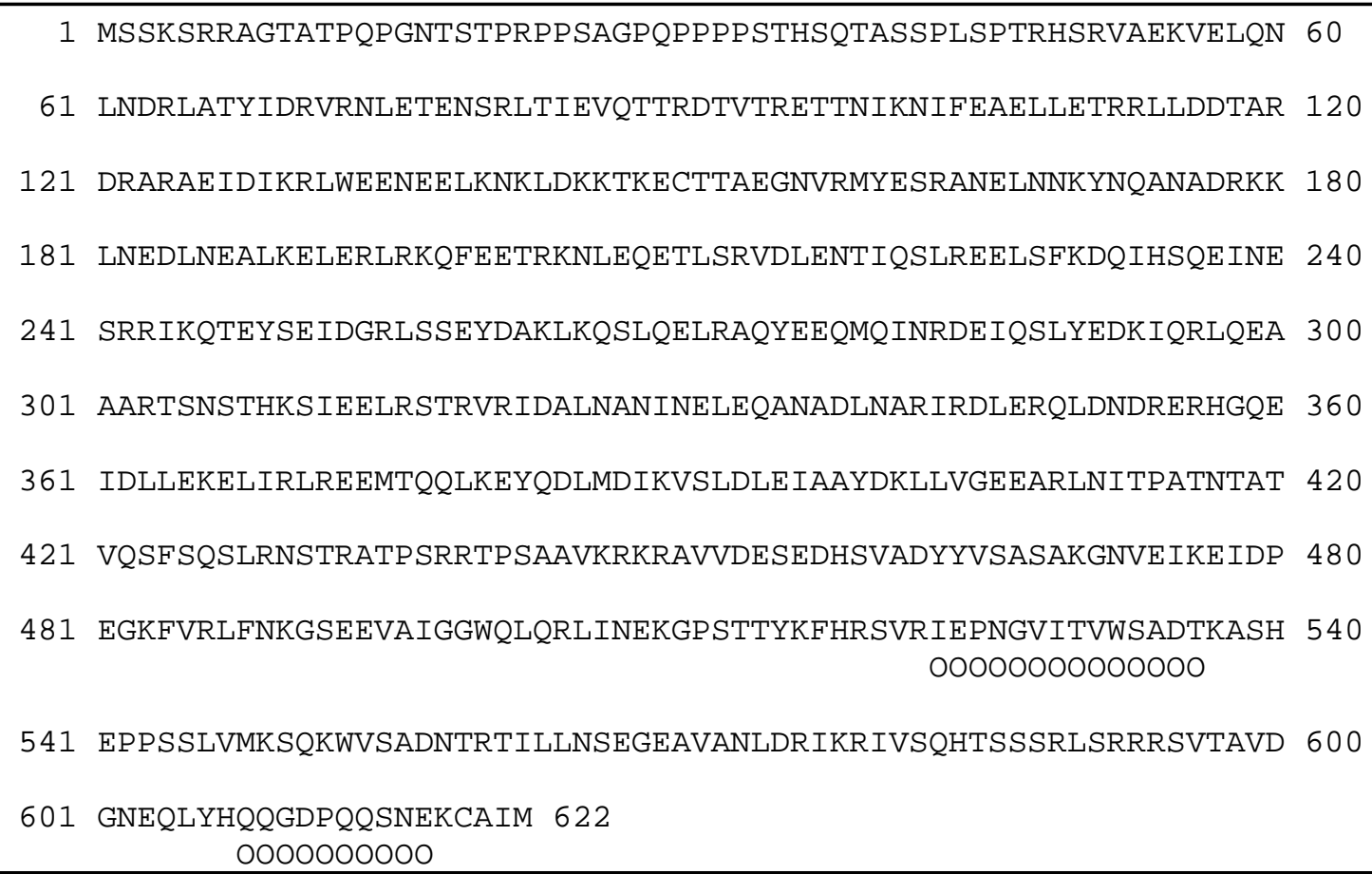

Supplement Figure 9: The amino acid sequence of lamin Dm0 contains no potential PEST-motif

Amino acid analysis of lamin Dm0 using the software epestfind led to the identification of two poor PEST-motifs with 14 amino acids between position 522 and 537 (PEST score: -11.79) and 10 amino acids between position 607 and 618 (PEST score -2.64).Both PEST-motifs have a score of $<+5$ and are below the threshold. 
Supplement Table: 1 : Top 50 candidate list: $\Delta 15$, embryo 6-8 hours

\begin{tabular}{|c|c|c|c|c|c|}
\hline group & gene id & gene name & Chr & function & $\begin{array}{l}\log 2 \\
\text { fold }\end{array}$ \\
\hline $\mathbf{0}$ & FBgn0051813 & & & & $-26,70$ \\
\hline $\mathbf{0}$ & FBgn0028855 & & & & $-23,81$ \\
\hline 0 & FBgn0051300 & CG31300 & $3 \mathrm{R}$ & $\begin{array}{l}\text { transferase activity, transferring } \\
\text { phosphorus-containing groups }\end{array}$ & $-23,75$ \\
\hline $\mathbf{0}$ & FBgn0033275 & CG14756 & $2 \mathrm{R}$ & & $-12,32$ \\
\hline $\mathbf{0}$ & FBgn0052475 & mthl8 & $3 \mathrm{~L}$ & G-protein coupled receptor activity & $-11,25$ \\
\hline $\mathbf{0}$ & FBgn0035208 & CG9184 & $3 \mathrm{~L}$ & & $-10,72$ \\
\hline $\mathbf{0}$ & FBgn0033721 & CG13159 & $2 \mathrm{R}$ & & $-10,57$ \\
\hline 3 & FBgn0039073 & & & & $-10,54$ \\
\hline $\mathbf{0}$ & FBgn0052640 & & & & $-9,99$ \\
\hline $\mathbf{0}$ & FBgn0035621 & CG10591 & $3 \mathrm{~L}$ & & $-9,77$ \\
\hline $2,4,5$ & FBgn0036789 & AstC-R2 & $3 \mathrm{~L}$ & neuropeptide receptor activity & $-9,72$ \\
\hline 3 & FBgn0052318 & CG32318 & $3 \mathrm{~L}$ & ATP binding & $-9,20$ \\
\hline $\mathbf{0}$ & FBgn0261341 & verm & $3 \mathrm{~L}$ & protein binding & $-9,17$ \\
\hline $\mathbf{0}$ & FBgn0029728 & CG2861 & $\mathrm{X}$ & & $-8,70$ \\
\hline $\mathbf{0}$ & FBgn0037447 & $\begin{array}{l}\text { Neurochond } \\
\text { rin }\end{array}$ & $3 R$ & binding & $-8,29$ \\
\hline 7 & FBgn0003710 & tipE & $3 \mathrm{~L}$ & plasma membrane & $-8,20$ \\
\hline 0 & FBgn0039795 & Spn100A & $3 \mathrm{R}$ & extracellular space & $-8,11$ \\
\hline 3 & FBgn0038610 & CG7675 & $3 \mathrm{R}$ & oxidoreductase activity & $-7,83$ \\
\hline $\mathbf{0}$ & FBgn0038581 & CG14314 & $3 \mathrm{R}$ & $\begin{array}{l}\text { transferase activity, transferring } \\
\text { phosphorus-containing groups }\end{array}$ & $-7,78$ \\
\hline $\mathbf{0}$ & FBgn0038476 & kuk & $3 R$ & lipid particle & $-7,60$ \\
\hline $\mathbf{0}$ & FBgn0035495 & CG14989 & $3 \mathrm{~L}$ & & $-7,48$ \\
\hline $\mathbf{0}$ & FBgn0003382 & sha & $2 \mathrm{R}$ & molecular_function & $-7,33$ \\
\hline 3,7 & FBgn0038734 & & & & $-7,32$ \\
\hline $\mathbf{0}$ & FBgn0031088 & CG15322 & $\mathrm{X}$ & & $-7,11$ \\
\hline 3 & FBgn0034756 & Cyp6d2 & $2 \mathrm{R}$ & oxidation-reduction process & $-6,84$ \\
\hline $\mathbf{0}$ & FBgn0260874 & Ir76a & $3 \mathrm{~L}$ & sensory dendrite & 4,91 \\
\hline 3,7 & FBgn0003996 & $\mathrm{w}$ & $\mathrm{X}$ & $\begin{array}{l}\text { transmembrane signaling receptor } \\
\text { activity }\end{array}$ & 4,97 \\
\hline 2 & FBgn0031461 & daw & $2 \mathrm{~L}$ & determination of adult lifespan & 5,12 \\
\hline 2 & FBgn0032484 & kek4 & $2 \mathrm{~L}$ & axonogenesis & 5,15 \\
\hline $\mathbf{0}$ & FBgn0262104 & CG42857 & $3 \mathrm{R}$ & cellular_component & 5,18 \\
\hline $\mathbf{0}$ & FBgn0265912 & & & & 5,24 \\
\hline 3,4 & FBgn0034085 & Ptp52F & $2 \mathrm{R}$ & protein binding & 5,24 \\
\hline 3,7 & FBgn0037973 & CG18547 & $3 \mathrm{R}$ & oxidoreductase activity & 5,43 \\
\hline $\mathbf{0}$ & FBgn0265533 & & & & 5,45 \\
\hline 1,3 & FBgn0001149 & GstD1 & $3 R$ & protein binding & 6,02 \\
\hline $\mathbf{0}$ & FBgn0038294 & Mf & $3 \mathrm{R}$ & chaeta development & 7,10 \\
\hline 3 & FBgn0015038 & Cyp9b1 & $2 \mathrm{R}$ & oxidation-reduction process & 7,15 \\
\hline $\mathbf{0}$ & FBgn0037408 & NPFR & $3 \mathrm{R}$ & integral component of membrane & 7,27 \\
\hline 6 & FBgn0037986 & CG14736 & $3 R$ & membrane & 7,50 \\
\hline 3,7 & FBgn0037975 & CG3397 & $3 \mathrm{R}$ & oxidoreductase activity & 7,69 \\
\hline 2,6 & FBgn0031585 & CG2955 & $2 \mathrm{~L}$ & protein binding & 7,70 \\
\hline $\mathbf{0}$ & FBgn0051286 & & & & 7,72 \\
\hline $\mathbf{0}$ & FBgn0052010 & & & & 8,01 \\
\hline $\mathbf{0}$ & FBgn0004911 & & & & 8,06 \\
\hline $\mathbf{0}$ & FBgn0267248 & & & & 8,13 \\
\hline 3 & FBgn0051683 & & & & 8,81 \\
\hline $\mathbf{0}$ & FBgn0266219 & & & & 8,91 \\
\hline $2,4,5,7$ & FBgn0038840 & CG5621 & $3 \mathrm{R}$ & cell junction & 10,17 \\
\hline 2 & FBgn0052581 & CG32581 & $\mathrm{X}$ & protein binding & 10,43 \\
\hline $\mathbf{0}$ & FBgn0038718 & & & & 11,35 \\
\hline
\end{tabular}


Supplement Table: 2: List of commercial available fly-lines used in the RNAi-screen

\begin{tabular}{|c|c|c|c|c|c|}
\hline & name & VDRC & & name & VDRC \\
\hline 1 & APC1 & 110290/KK II & 69 & Lamin Dm0 & 107419 \\
\hline 2 & Axs & 6137 & 70 & Lamin ds RNA & 45635 \\
\hline 3 & baf & 102013 & 71 & lamin ds RNA & 107419 \\
\hline 4 & $\begin{array}{l}\text { Bcell associated } \\
\text { protein3 }\end{array}$ & KK106452 & 72 & LaminDm0 & 45635 \\
\hline 5 & bocksbeurtel & 24189 & 73 & LAP1 & 18600/80 II \\
\hline 6 & bocksbeutel & 24191 & 74 & LAS1 & KK105415 \\
\hline 7 & btz, barentsz & 38722 & 75 & LBR & 39468 \\
\hline 8 & Caf-1 180 & 20151 & 76 & LBR & 110508/KK II \\
\hline 9 & Caf-1, p55 & 26455 & 77 & Lipin & 107707/KK II \\
\hline 10 & CdsA & 5121 & 78 & Lk6 & 32885 \\
\hline 11 & CG12013 & 100790/KK & 79 & Mad & 12635 \\
\hline 12 & CG1274 & 104006/KK & 80 & Mad & 110517/KK II \\
\hline 13 & CG13887 & 106452/KK & 81 & MAN1 & 108906/KK II \\
\hline 14 & CG14444 & 110678/KK & 82 & mbo & 47693 \\
\hline 15 & CG14696 & 100232/KK & 83 & msp-300 & 50192 \\
\hline 16 & CG16944 & 104576/KK & 84 & MSP300 & 107183/KK \\
\hline 17 & CG1742 & 109140/KK & 85 & Mtor & 24265 \\
\hline 18 & CG17991 & 1277 & 86 & Mtor & 24265 \\
\hline 19 & CG31460 & 103626/KK & 87 & n.a. & 21604 \\
\hline 20 & CG3149 & 47560 & 88 & n.a. & 1277 \\
\hline 21 & CG3167 & 108906/KK & 89 & Ndc1 & 3408 \\
\hline 22 & CG32297 & 34506 & 90 & Ndc1 & 101264/KK \\
\hline 23 & CG33129 & 107365/KK & 91 & Nesprin & 107183/KK II \\
\hline 24 & CG44154 & 108236/KK & 92 & Notch & 27229 (80) III \\
\hline 25 & CG44254 & 109119/KK & 93 & Notch & 100002/KK II \\
\hline 26 & CG6136 & 109421/KK & 94 & Notch & 27229 \\
\hline 27 & CG6743 & 110759/KK & 95 & nucleophosmin & KK102920 \\
\hline 28 & CG6773 & 110428/KK & 96 & nucleoplasmin & GD22623 \\
\hline 29 & CG6961 & 109951/KK & 97 & Nup 50 & 100564 \\
\hline 30 & CG6962 & KK110163 & 98 & Nup107 & 22407 \\
\hline 31 & CG7830 & 105649/KK & 99 & Nup153 & 47155 \\
\hline 32 & CG8111 & 101348/KK & 100 & Nup153 & 2340 \\
\hline 33 & CG8112 & $100347 / \mathrm{KK}$ & 101 & Nxt1 & 52631 \\
\hline 34 & CG8679 & 30778 & 102 & Otefin & 105308 \\
\hline 35 & CG8679 & 30778 & 103 & Otefin & 1095308/KK II \\
\hline 36 & CG9159 & 109910/KK & 104 & p53 & 38235 \\
\hline 37 & CG9323 & 44984 & 105 & p53 & 10692 \\
\hline 38 & CG9662 & 100769/KK & 106 & p53 & 38235 \\
\hline 39 & cup & 18179 & 107 & P66 Simyang & 13066 \\
\hline
\end{tabular}




\begin{tabular}{|c|c|c|c|c|c|}
\hline 40 & Cup & 18179 & 108 & p66, simjang & 13066 \\
\hline 41 & Delta & 109491/KK II & 109 & $\begin{array}{l}\text { Phosphatidyl- } \\
\text { inositolglycan } \\
\text { anchor }\end{array}$ & KK107643 \\
\hline 42 & Delta & 109491 & 110 & picolo & GD44974 \\
\hline 43 & D-MAN1 & 11149 & 111 & Pnt & 105390/KK \\
\hline 44 & dMyc & 106066/KK & 112 & Pontin/Ruv BL1 & KK105408 \\
\hline 45 & dMyc & 2947/GD & 113 & prohibitin & GD12360 \\
\hline 46 & $\mathrm{E}(\mathrm{bx})$ & 46645 & 114 & prohibitin 2 & GD32361 \\
\hline 47 & EGFR & 107130/KK & 115 & quemao & 47001 \\
\hline 48 & erlin 2A & GD44273 & 116 & Reptin & KK103483 \\
\hline 49 & erlin 2B & KK 101392 & 117 & RFC2 & KK108452 \\
\hline 50 & fs(1)YA & 12232 & 118 & $\mathrm{sbr}$ & 32690 \\
\hline 51 & Fs(2)Ket & 22348 & 119 & Scalloped & 101497/KK \\
\hline 52 & Ftase & 47000 & 120 & SHMT & 19206 \\
\hline 53 & Ftase & 47001 & 121 & STAT92E & 43867 \\
\hline 54 & GCL & 28897 & 122 & $\mathrm{Su}(\mathrm{H})$ & 103597/KK II \\
\hline 55 & GV1 & 107768 & 123 & Su(var)2-10 & 30709 \\
\hline 56 & Hairless & 100046 & 124 & Su(Var)3-9 & 33834 \\
\hline 57 & Hopscotch & 40037 & 125 & Su(Var)3-9 & 39377 \\
\hline 58 & HP1aSu(var)2-5 & 31995 & 126 & TMEM 209 & GD45648 \\
\hline 59 & HP1b & 26097 & 127 & TMEM194A & 105905 \\
\hline 60 & HP1c & 50520 & 128 & TMEM209 & KK105105 \\
\hline 61 & Hp1c & 27782 & 129 & Ugt1 & 16467 \\
\hline 62 & iswi & 6208 & 130 & Ulp1 & 31744 \\
\hline 63 & Klar & 32836 & 131 & ulp1 & 106625 \\
\hline 64 & Klaroid & $3990 / 80$ & 132 & WD repeat 18 & KK105616 \\
\hline 65 & Klaroid & 108236/KK & 133 & Wts & 9928/80 \\
\hline 66 & Klarsicht & $32836 / 80$ & 134 & Wts & 106174/KK \\
\hline 67 & Kuk & 106855/KK II & 135 & Y37H913 & 21928 \\
\hline 68 & kuk ds RNA & 34959 & 136 & Yortz barentsz & 38722 \\
\hline
\end{tabular}


Supplement Table: 3: Top 50 candidate list - Narf WT

MHC-GS Gal4; UAS-Narf WT + $200 \mu \mathrm{M}$ RU, Thorax sample $10 \mathrm{~d}$

\begin{tabular}{|c|c|c|c|c|c|}
\hline group & gene_id & gene_name & Chr & function & $\begin{array}{l}\text { log2 } \\
\text { fold }\end{array}$ \\
\hline 1 & FBgn0262115 & CG17683 & $2 \mathrm{R}$ & neurogenesis & 7,08 \\
\hline 1 & FBgn0004034 & $\mathrm{y}$ & $\mathrm{X}$ & cytoplasm & 5,24 \\
\hline 1 & FBgn0033395 & Сур4p2 & $2 \mathrm{R}$ & oxidation-reduction process & 4,59 \\
\hline 1 & FBgn0052640 & CG32640 & $\mathrm{X}$ & response to heat & 4,45 \\
\hline $\mathbf{0}$ & FBgn0037964 & CG14731 & $3 R$ & metabolic process & 3,92 \\
\hline 1 & FBgn0266310 & & & & 3,87 \\
\hline 1 & FBgn0263744 & & & & 3,70 \\
\hline 1 & FBgn0262430 & & & & 3,65 \\
\hline 1 & FBgn0003082 & phr & $2 \mathrm{R}$ & DNA repair & 3,56 \\
\hline 1 & FBgn0266094 & & & & 3,53 \\
\hline $\mathbf{0}$ & FBgn0267680 & & & & 3,46 \\
\hline $\mathbf{0}$ & FBgn0053160 & CG33160 & 3L & proteolysis & 3,40 \\
\hline $\mathbf{0}$ & FBgn0262789 & & & & 3,25 \\
\hline 1 & FBgn0262386 & & & & 3,14 \\
\hline $\mathbf{0}$ & FBgn0037972 & CG10005 & $3 \mathrm{R}$ & cellular_component & 3,11 \\
\hline $\mathbf{0}$ & FBgn0053159 & CG33159 & $3 \mathrm{~L}$ & proteolysis & 3,07 \\
\hline $\mathbf{0}$ & FBgn0004379 & Klp67A & $3 \mathrm{~L}$ & mitotic spindle organization & 2,98 \\
\hline 1 & FBgn0032551 & CG18636 & $2 \mathrm{~L}$ & proteolysis & 2,96 \\
\hline 1 & FBgn0042102 & CG18745 & $3 R$ & & 2,93 \\
\hline 1 & FBgn0038353 & CG5399 & $3 \mathrm{R}$ & & 2,93 \\
\hline $\mathbf{0}$ & FBgn0262452 & & & & 2,89 \\
\hline 1 & FBgn0262413 & & & & 2,85 \\
\hline $\mathbf{0}$ & FBgn0052277 & CG32277 & 3L & proteolysis & 2,83 \\
\hline 1 & FBgn0038391 & GATAe & $3 R$ & $\begin{array}{l}\text { RNA polymerase II transcription } \\
\text { factor activity }\end{array}$ & 2,83 \\
\hline $\mathbf{0}$ & FBgn0265186 & CG44251 & $2 \mathrm{R}$ & & 2,80 \\
\hline $\mathbf{0}$ & FBgn0264569 & & & & $-2,52$ \\
\hline $\mathbf{0}$ & FBgn0265949 & & & & $-2,52$ \\
\hline $\mathbf{0}$ & FBgn0050287 & CG30287 & 2R & proteolysis & $-2,52$ \\
\hline $\mathbf{0}$ & FBgn0264810 & Pburs & $2 \mathrm{~L}$ & $\begin{array}{l}\text { G-protein coupled receptor } \\
\text { signaling pathway }\end{array}$ & $-2,52$ \\
\hline $\mathbf{0}$ & FBgn0040261 & Ugt36Bb & $2 \mathrm{~L}$ & metabolic process & $-2,54$ \\
\hline $\mathbf{0}$ & FBgn0069354 & Porin2 & 2L & regulation of anion transport & $-2,55$ \\
\hline 1 & FBgn0040359 & CG11380 & $\mathrm{X}$ & & $-2,55$ \\
\hline $\mathbf{0}$ & FBgn0038191 & CG9925 & 3R & zinc ion binding & $-2,58$ \\
\hline $\mathbf{0}$ & FBgn0032804 & CG13081 & $2 \mathrm{~L}$ & cellular_component & $-2,61$ \\
\hline 1 & FBgn0033628 & CG13203 & $2 \mathrm{R}$ & & $-2,68$ \\
\hline $\mathbf{0}$ & FBgn0050395 & CG30395 & $2 \mathrm{R}$ & molecular_function & $-2,74$ \\
\hline 1 & FBgn0265813 & & & & $-2,88$ \\
\hline 1 & FBgn0039009 & CG13842 & $3 R$ & & $-2,94$ \\
\hline 1 & FBgn0025776 & ind & $3 \mathrm{~L}$ & nucleus & $-2,96$ \\
\hline 1 & FBgn0262150 & CG42876 & $2 \mathrm{~L}$ & & $-3,11$ \\
\hline $\mathbf{0}$ & FBgn0031559 & CG3513 & $2 \mathrm{~L}$ & $\begin{array}{ll}\text { serine-type } & \text { endopeptidase } \\
\text { inhibitor activity } & \end{array}$ & $-3,18$ \\
\hline 1 & FBgn0038315 & CG14866 & $3 \mathrm{R}$ & carbohydrate binding & $-3,19$ \\
\hline 1 & FBgn0263250 & CG43393 & $2 \mathrm{R}$ & cellular_component & $-3,41$ \\
\hline 1 & FBgn0053302 & Cpr31A & $2 \mathrm{~L}$ & $\begin{array}{l}\text { structural constituent of chitin- } \\
\text { based cuticle }\end{array}$ & $-3,43$ \\
\hline 1 & FBgn0266156 & & & & $-3,46$ \\
\hline 1 & FBgn0050121 & & & & $-3,50$ \\
\hline 1 & FBgn0030105 & CG15369 & $\mathrm{X}$ & $\begin{array}{l}\text { cysteine-type } \\
\text { inhibitor activity }\end{array}$ & $-3,56$ \\
\hline
\end{tabular}




\begin{tabular}{|c|c|c|c|c|c|}
\hline 1 & FBgn0262721 & CG43165 & $2 \mathrm{~L}$ & $\begin{array}{ll}\text { serine-type } & \text { endopeptidase } \\
\text { inhibitor activity } & \end{array}$ & $-3,60$ \\
\hline 1 & FBgn0058469 & & & & $-5,53$ \\
\hline 1 & FBgn0265912 & & & & $-6,52$ \\
\hline
\end{tabular}

Supplement Table: 4: Top 50 candidate list - Narf H/R

MHC-GS Gal4; UAS-Narf H/R + $200 \mu \mathrm{M}$ RU, Thorax sample $10 \mathrm{~d}$

\begin{tabular}{|c|c|c|c|c|c|}
\hline group & gene_id & gene_name & Chr. & function & $\begin{array}{l}\text { log2 } \\
\text { fold }\end{array}$ \\
\hline 1 & FBgn0262115 & CG17683 & $2 \mathrm{R}$ & neurogenesis & 6,60 \\
\hline 1 & FBgn0004034 & $\mathrm{y}$ & $\mathrm{X}$ & cytoplasm & 5,68 \\
\hline 1 & FBgn0033395 & Сур4p2 & $2 \mathrm{R}$ & oxidation-reduction process & 4,64 \\
\hline 1 & FBgn0052640 & CG32640 & $X$ & response to heat & 4,49 \\
\hline 1 & FBgn0003082 & phr & $2 \mathrm{R}$ & DNA repair & 3,71 \\
\hline $\mathbf{0}$ & FBgn0026760 & Tehao & $2 \mathrm{~L}$ & protein binding & 3,71 \\
\hline 1 & FBgn0266310 & & & & 3,64 \\
\hline 1 & FBgn0263744 & & & & 3,51 \\
\hline $\mathbf{0}$ & FBgn0050049 & CG30049 & $2 \mathrm{R}$ & cellular_component & 3,30 \\
\hline 1 & FBgn0042102 & CG18745 & $3 \mathrm{R}$ & & 3,26 \\
\hline $\mathbf{0}$ & FBgn0013692 & & & & 3,24 \\
\hline 1 & FBgn0038353 & CG5399 & 3R & & 3,22 \\
\hline 1 & FBgn0262430 & & & & 3,21 \\
\hline $\mathbf{0}$ & FBgn0036419 & CG13482 & 3L & & 3,12 \\
\hline 1 & FBgn0266094 & & & & 3,06 \\
\hline 1 & FBgn0038391 & GATAe & $3 R$ & $\begin{array}{l}\text { RNA polymerase II transcription } \\
\text { factor activity }\end{array}$ & 2,99 \\
\hline $\mathbf{0}$ & FBgn0002570 & Mal-A1 & $2 \mathrm{R}$ & cation binding & 2,97 \\
\hline $\mathbf{0}$ & FBgn0040299 & Myo28B1 & $2 \mathrm{~L}$ & protein binding & 2,97 \\
\hline 1 & FBgn0032551 & CG18636 & $2 \mathrm{~L}$ & proteolysis & 2,94 \\
\hline 1 & FBgn0262413 & & & & 2,93 \\
\hline $\mathbf{0}$ & FBgn0034294 & Muc55B & $2 \mathrm{R}$ & biological_process & 2,88 \\
\hline $\mathbf{0}$ & FBgn0040363 & CG11384 & $\mathrm{X}$ & integral component of membrane & 2,84 \\
\hline $\mathbf{0}$ & FBgn0030756 & CG9903 & $\mathrm{X}$ & membrane & 2,81 \\
\hline 1 & FBgn0262386 & & & & 2,78 \\
\hline $\mathbf{0}$ & FBgn0265104 & & & & 2,76 \\
\hline $\mathbf{0}$ & FBgn0016974 & & & & $-2,67$ \\
\hline 1 & FBgn0025776 & ind & 3L & nucleus & $-2,67$ \\
\hline $\mathbf{0}$ & FBgn0083946 & lobo & $3 \mathrm{R}$ & molecular_function & $-2,70$ \\
\hline 1 & FBgn0033628 & CG13203 & $2 \mathrm{R}$ & & $-2,70$ \\
\hline $\mathbf{0}$ & FBgn0036410 & CG8100 & 3L & extracellular region & $-2,71$ \\
\hline $\mathbf{0}$ & FBgn0051025 & Ppi1 & $3 \mathrm{R}$ & protein phosphatase 1 binding & $-2,71$ \\
\hline $\mathbf{0}$ & FBgn0039469 & TwdlC & $3 R$ & $\begin{array}{l}\text { structural constituent of chitin- } \\
\text { based cuticle }\end{array}$ & $-2,73$ \\
\hline 1 & FBgn0262150 & CG42876 & $2 \mathrm{~L}$ & & $-2,76$ \\
\hline $\mathbf{0}$ & FBgn0267728 & otk2 & $2 \mathrm{R}$ & protein binding & $-2,80$ \\
\hline $\mathbf{0}$ & FBgn0029848 & Btd & $\mathrm{X}$ & histone biotinylation & $-2,81$ \\
\hline $\mathbf{0}$ & FBgn0037078 & CG12971 & $3 \mathrm{~L}$ & cellular_component & $-2,88$ \\
\hline 1 & FBgn0030105 & CG15369 & $\mathrm{X}$ & $\begin{array}{ll}\text { cysteine-type } & \text { endopeptidase } \\
\text { inhibitor activity } & \end{array}$ & $-2,90$ \\
\hline 1 & FBgn0039009 & CG13842 & $3 \mathrm{R}$ & & $-2,90$ \\
\hline $\mathbf{0}$ & FBgn0033597 & Cpr47Ea & $2 \mathrm{R}$ & $\begin{array}{l}\text { structural constituent of chitin- } \\
\text { based cuticle }\end{array}$ & $-2,91$ \\
\hline 1 & FBgn0262721 & CG43165 & $2 \mathrm{~L}$ & $\begin{array}{l}\text { serine-type } \\
\text { inhibitor activity }\end{array}$ & $-2,91$ \\
\hline $\mathbf{0}$ & FBgn0037323 & CG2663 & 3R & transporter activity & $-2,94$ \\
\hline 1 & FBgn0265813 & & & & $-3,02$ \\
\hline
\end{tabular}




\begin{tabular}{|r|l|l|l|l|r|}
\hline $\mathbf{1}$ & FBgn0040359 & CG11380 & X & & $-3,19$ \\
\hline $\mathbf{1}$ & FBgn0263250 & CG43393 & 2R & cellular_component & $-3,27$ \\
\hline $\mathbf{1}$ & FBgn0050121 & & & & $-3,47$ \\
\hline $\mathbf{1}$ & FBgn0038315 & CG14866 & 3R & carbohydrate binding & $-3,60$ \\
\hline $\mathbf{1}$ & FBgn0053302 & Cpr31A & 2L & $\begin{array}{l}\text { structural constituent of chitin- } \\
\text { based cuticle }\end{array}$ & $-3,66$ \\
\hline $\mathbf{1}$ & FBgn0266156 & & & & $-3,78$ \\
\hline $\mathbf{1}$ & FBgn0058469 & & & & $-5,93$ \\
\hline 1 & FBgn0265912 & & & & $-6,11$ \\
\hline
\end{tabular}

Supplement Table: 5: Top 50 candidate list - Narf RNAi

MHC-GS Gal4; UAS-Narf RNAi + $20 \mu \mathrm{M}$ RU, Thorax sample $10 \mathrm{~d}$

\begin{tabular}{|c|c|c|c|c|c|}
\hline group & gene_id & gene_name & Chr & function & $\begin{array}{l}\log 2 \\
\text { fold }\end{array}$ \\
\hline $\mathbf{0}$ & FBgn0262811 & CG43182 & $\mathrm{X}$ & cellular_component & $-8,77$ \\
\hline 1 & FBgn0004034 & $\mathrm{y}$ & $\mathrm{X}$ & cytoplasm & $-6,61$ \\
\hline $\mathbf{0}$ & FBgn0053503 & Cyp12d1-d & 2R & mitochondrion & $-6,58$ \\
\hline $\mathbf{0}$ & FBgn0266822 & & & & $-5,29$ \\
\hline $\mathbf{0}$ & FBgn0003996 & $\mathrm{W}$ & $\mathrm{X}$ & $\begin{array}{l}\text { transmembrane signaling receptor } \\
\text { activity }\end{array}$ & $-5,01$ \\
\hline 1 & FBgn0052640 & CG32640 & $\mathrm{X}$ & response to heat & $-4,86$ \\
\hline $\mathbf{0}$ & FBgn0032968 & CG11634 & 2L & cellular_component & $-4,33$ \\
\hline 1 & FBgn0266253 & & & & $-4,32$ \\
\hline $\mathbf{0}$ & FBgn0262945 & & & & $-4,30$ \\
\hline 1 & FBgn0039299 & CG11854 & $3 R$ & extracellular space & $-4,23$ \\
\hline 1 & FBgn0036419 & CG13482 & 3L & & $-4,17$ \\
\hline 1 & FBgn0034293 & CG14495 & $2 \mathrm{R}$ & & $-4,12$ \\
\hline 1 & FBgn0265186 & CG44251 & $2 \mathrm{R}$ & & $-4,00$ \\
\hline $\mathbf{0}$ & FBgn0051600 & CG31600 & $2 \mathrm{~L}$ & cellular_component & $-3,82$ \\
\hline $\mathbf{0}$ & FBgn0262007 & CG42825 & 3L & integral component of membrane & $-3,71$ \\
\hline 1 & FBgn0003082 & phr & $2 \mathrm{R}$ & DNA repair & $-3,67$ \\
\hline $\mathbf{0}$ & FBgn0004191 & & & & $-3,62$ \\
\hline 1 & FBgn0039298 & to & $3 R$ & molecular_function & $-3,57$ \\
\hline 1 & FBgn0032235 & CG5096 & $2 \mathrm{~L}$ & protein binding & $-3,51$ \\
\hline 1 & FBgn0042102 & CG18745 & $3 R$ & & $-3,40$ \\
\hline $\mathbf{0}$ & FBgn0053532 & lectin-37Da & $2 \mathrm{~L}$ & extracellular region & $-3,38$ \\
\hline 1 & FBgn0265185 & CG44250 & $2 \mathrm{R}$ & & $-3,36$ \\
\hline $\mathbf{0}$ & FBgn0033733 & CG8834 & $2 \mathrm{R}$ & metabolic process & $-3,34$ \\
\hline 1 & FBgn0034031 & CG12963 & $2 \mathrm{R}$ & & $-3,34$ \\
\hline 1 & FBgn0036320 & CG10943 & 3L & & $-3,33$ \\
\hline 1 & FBgn0085410 & TrissinR & $2 \mathrm{~L}$ & $\begin{array}{l}\text { G-protein coupled receptor } \\
\text { activity }\end{array}$ & 3,18 \\
\hline $\mathbf{0}$ & FBgn0035865 & CG7201 & 3L & & 3,22 \\
\hline $\mathbf{0}$ & FBgn0002609 & $\begin{array}{l}\text { E(spl)m3- } \\
\text { HLH }\end{array}$ & $3 R$ & nucleus & 3,23 \\
\hline 1 & FBgn0262000 & CG42818 & $2 \mathrm{~L}$ & & 3,25 \\
\hline 1 & FBgn0034994 & Ir60a & $2 \mathrm{R}$ & integral component of membrane & 3,30 \\
\hline 1 & FBgn0036790 & AstC-R1 & 3L & integral component of membrane & 3,30 \\
\hline $\mathbf{0}$ & FBgn0030204 & Or9a & $\mathrm{X}$ & integral component of membrane & 3,36 \\
\hline 1 & FBgn0266899 & & & & 3,41 \\
\hline $\mathbf{0}$ & FBgn0266842 & & & & 3,44 \\
\hline 1 & FBgn0032337 & AstCC & $2 \mathrm{~L}$ & hormone activity & 3,48 \\
\hline 1 & FBgn0267728 & otk2 & $2 \mathrm{R}$ & protein binding & 3,50 \\
\hline 1 & FBgn0267727 & Pen & $2 \mathrm{~L}$ & binding & 3,50 \\
\hline $\mathbf{0}$ & FBgn0038221 & CG3259 & $3 R$ & cilium & 3,51 \\
\hline 1 & FBgn0083946 & lobo & $3 R$ & molecular_function & 3,51 \\
\hline
\end{tabular}




\begin{tabular}{|c|c|c|c|c|c|}
\hline 1 & FBgn0051025 & Ppi1 & 3R & protein phosphatase 1 binding & 3,55 \\
\hline 1 & FBgn0022981 & rpk & $3 \mathrm{R}$ & integral component of membrane & 3,69 \\
\hline 1 & FBgn0030105 & CG15369 & $\mathrm{X}$ & $\begin{array}{ll}\text { cysteine-type } & \text { endopeptidase } \\
\text { inhibitor activity } & \end{array}$ & 3,71 \\
\hline $\mathbf{0}$ & FBgn0051683 & CG31683 & 2L & lipid metabolic process & 3,87 \\
\hline $\mathbf{0}$ & FBgn0035880 & CG17352 & $3 \mathrm{~L}$ & early endosome & 3,93 \\
\hline $\mathbf{0}$ & FBgn0038915 & CG17819 & 3R & GTP binding & 4,02 \\
\hline 1 & FBgn0053302 & Cpr31A & $2 \mathrm{~L}$ & $\begin{array}{l}\text { structural constituent of chitin- } \\
\text { based cuticle }\end{array}$ & 4,15 \\
\hline 1 & FBgn0263250 & CG43393 & $2 \mathrm{R}$ & cellular_component & 4,33 \\
\hline 1 & FBgn0058469 & & & & 5,14 \\
\hline 1 & FBgn0265912 & & & & 5,97 \\
\hline $\mathbf{0}$ & FBgn0052475 & mthl8 & 3L & $\begin{array}{l}\text { G-protein coupled receptor } \\
\text { activity }\end{array}$ & 7,11 \\
\hline
\end{tabular}

\section{Supplement Table description information:}

Supplement Table: 1:

group 1 - antioxidant activity

group 2 - binding

group 3 - catalytic activity

group 4 - reporter activity

group 5 - signal transducer activity

group 6 - structural molecule activity

group 7 - transporter activity

\section{Supplement Table 3/4:}

group 0 - Candidate was specifically identified for one overexpressed Narf allele group 0 - Candidate was identified within top50 lists of one Narf allele and can be found within total list of the other Narf allele

group 1 - Candidate was identified within top50 lists of both Narf alleles

Supplement Table: 5

group 0 - Candidate was specifically identified for the Narf knockdown situation group 1 - Candidate was also identified within total lists of one or both overexpressed Narf alleles 


\section{List of Figures}

Figure 1: The deletion of a kuk deficient line comprise $5.5 \mathrm{~kb}$ within the genomic locus...... $17-$

Figure 2: The kuk deficient line $\Delta 15$ represents a protein null allele $-18-$

Figure 3: Differentially expressed genes in kuk-depleted embryos $-20-$

Figure 4: kuk-depleted animals show a decreased survival rate and lifespan $-21-$

Figure 5: Genomic GFP-Kuk transgenes did not rescue the kuk-depleted phenotype....- 23 -

Figure 6: Protein degradation occurs at the C-terminal end of GFP-Kuk $-24-$

Figure 7: UAS/Gal4 expressed GFP-Kuk rescued the kuk depletion phenotype $25-$

Figure 8: Solubilization promoted GFP-Kuk degradation $-27-$

Figure 9: FRAP analysis of wild type and mutant GFP Kuk $-29-$

Figure 10: Scheme of the performed RNAi-screen $-31-$

Figure 11: Kugelkern overexpression caused wing morphological defects. - 32 -

Figure 12: Kuk overexpression induced eye-defects primary independent of apoptosis - 34 -

Figure 13: Genetic interaction partners of kuk remain unidentified ...............................- 36 -

Figure 14: Protein expression and purification of QE80N60-Narf .................................- 38 -

Figure 15: The antibody stained specific for Narf........................................................... 39 -

Figure 16: Narf localized to the nuclear envelope............................................................ 41 -

Figure 17: The lethal alleles NC38 and NC109 contained the described mutations........- 42 -

Figure 18: Characterization of different Narf lethal alleles........................................... 43 -

Figure 19: Narf protein level is decreased dramatically in NC38 homozygous larvae...- 44 -

Figure 20: Position of the human patient situation is conserved in Drosophila...............- 45 -

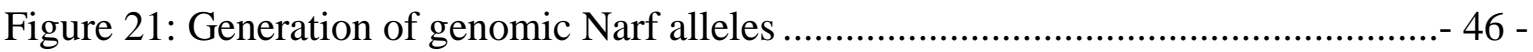

Figure 22: Overexpression of genomic Narf increased survival rate .............................. 48 -

Figure 23: Ubiquitous overexpression of Narf prolonged Drosophila lifespan ............... 49 -

Figure 24: Generation of a conditional Narf null allele by CRISPR/Cas9....................... 52 -

Figure 25: The GeneSwitch system enables spatial and dose-dependent gene expression..... $-54-$

Figure 26: The GeneSwitch Gal4 system enabled Narf overexpression .......................... 56 -

Figure 27: Narf overexpression affected nuclear size .................................................. 58 -

Figure 28: Narf overexpression might affect negative geotaxis behavior........................ 60 -

Figure 29: RU468 treatment did not affect Drosophila lifespan ..................................... 61 -

Figure 30: Narf overexpression during adulthood affected Drosophila lifespan ............. 63 - 
Figure 31: Narf knock-down did not affect nuclear size ................................................. 65 -

Figure 32: Muscle specific knockdown of Narf decreased lifespan $-66-$

Figure 33: Ubiquitous down regulation of Narf during adulthood severely affected survival rate $68-$

Figure 34: Gene expression is changed in altered Narf expressing situations $-70-$

Figure 35: oxy-4 mutants were sensitive to oxidative stress $-73-$

Figure 36: Developmental delay of oxy-4 mutants under normoxic conditions $-74-$

Figure 37: Striated muscle structure was normal in oxy-4 mutant worms $-75-$

Figure 38: oxy-4 mutants showed a decline in pharyngeal pumping. $-76-$

Figure 39: oxy-4 mutant worms were normal in non-striated muscle structure. $-76-$

Figure 40: Nuclear morphology was not affected in oxy-4 mutant worms at larval stag- 78 -

Figure 41: A lack of farnesylation in Ce-lamin did not result in a protein shift. $-79-$

Figure 42: Inefficient knockdown of oxy-4 $80-$

Figure 43: Lifespan analyses of oxy-4 mutants with additional lmn-1 RNAi treatment - 81 -

Figure 44: Amino acid sequence used for Nup153 antibody . $-122-$

Figure 45: Expression and purification of the pGEX-60H-Nup153 protein $-123-$

Figure 46: Specificity of the generated Nup153 antibody $-123-$

Figure 47: Nup153 antibody stains specific nuclear pore complexes - 124 -

Supplement Figure 1: The deletion of $\Delta 15$ a kuk deficient fly line

Supplement Figure 2: Kugelkern is stable for at least four hours after solubilization ..- 145 -

Supplement Figure 3: Kugelkern overexpression did not induce apoptosis in larval eye-discs $-146-$

Supplement Figure 4: Strongest Narf expression was achieved using the tubulin-Gal4 driver.

Supplement Figure 5: Wing specific down-regulation of Narf induced morphological defects $-147-$

Supplement Figure 6: Narf-RNAi efficiently down regulated larval expressed Narf ...- 147 -

Supplement Figure 7: p62/Ref(2)P protein level is not affected upon altered Narf expression $-148-$

Supplement Figure 8: The amino acid sequence of Kuk contains two potential PEST-motifs $148-$

Supplement Figure 9: The amino acid sequence of lamin Dm0 contains no potential - 149 


\section{List of Tables}

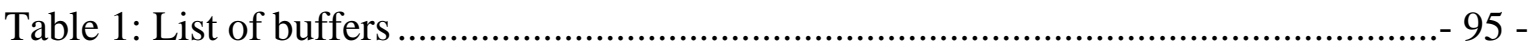

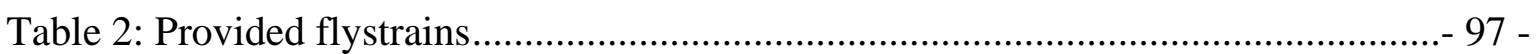

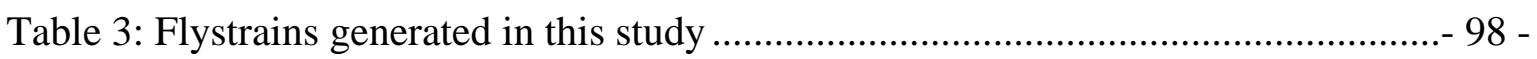

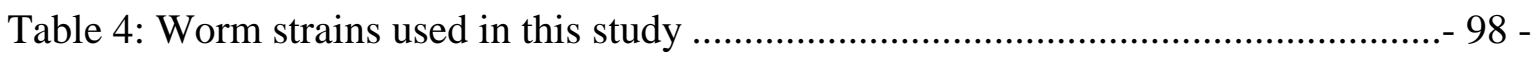

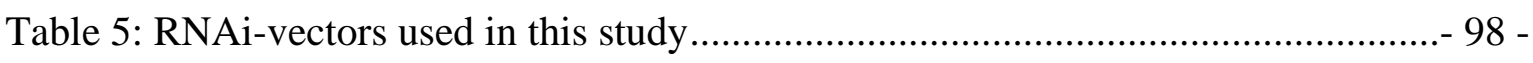

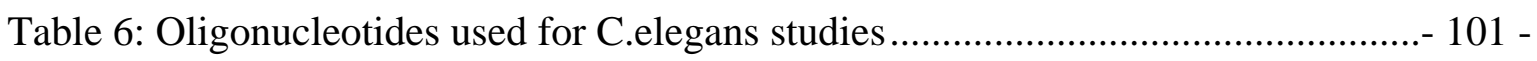

Table 7: Oligonucleotides used for Drosophila studies................................................- 101 -

Table 8: Provided plasmids used in this study ............................................................. 103 -

Table 9: Plasmid constructs generated in this study..................................................... 104 -

Supplement Table: 1: Top 50 candidate list: $\Delta 15$, embryo 6-8 hours ............................- 150 -

Supplement Table: 2: List of commercial available fly-lines used in the RNAi-screen- 151 -

Supplement Table: 3: Top 50 candidate list - Narf WT..............................................- 153 -

Supplement Table: 4: Top 50 candidate list - Narf H/R .............................................- 154 -

Supplement Table: 5: Top 50 candidate list - Narf RNAi ...........................................- 155 - 